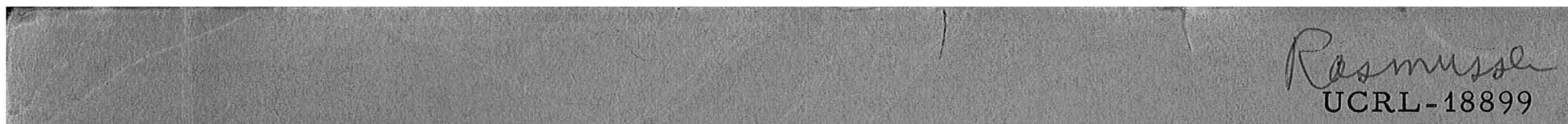

\title{
ON THE MICROSCOPIC AND MACROSCOPIC ASPECTS OF NUCLEAR STRUCTURE WITH APPLICATIONS TO SUPERHEA VY NUCLEI
}

Chin-Fu Tsang

(Ph.D. Thesis)

AEC Contract No. W $-7405-$ eng -48
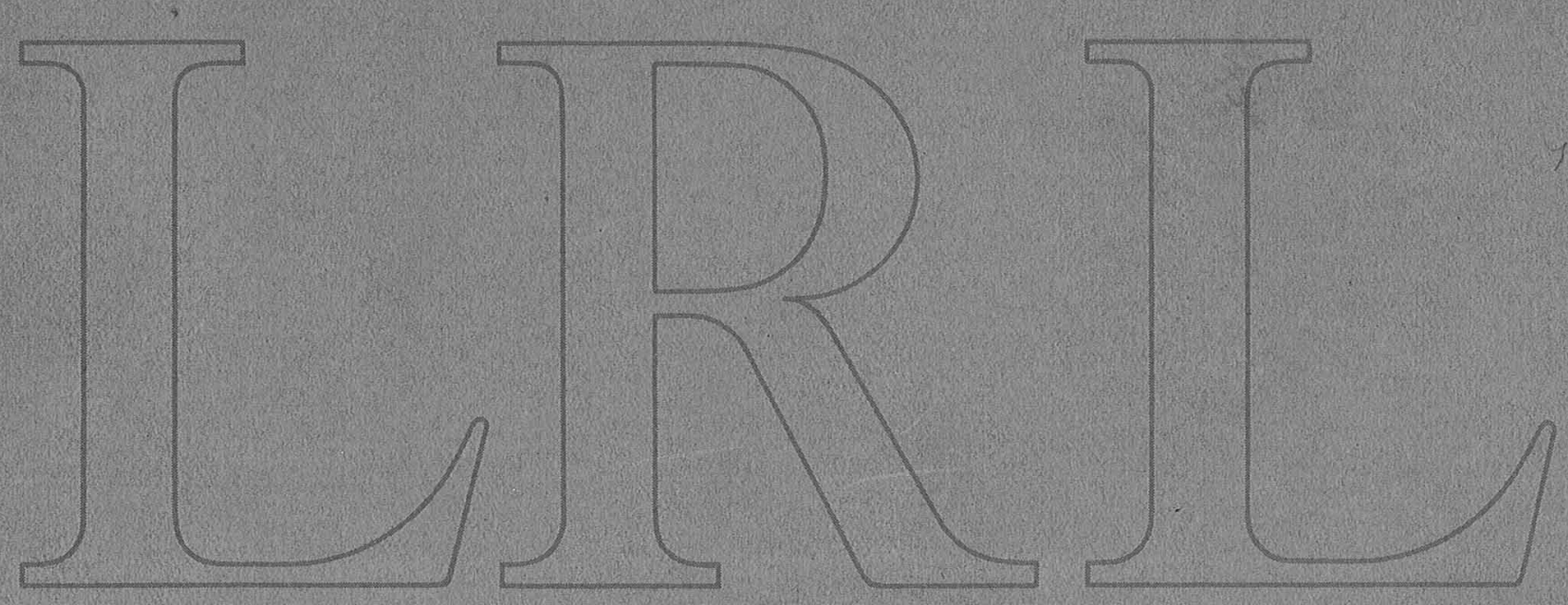

LAWRENCE RADIATION LABORATORY UNIVERSITY of CALIFORNIA BERKELEY 
Abstract . . . . . . . . . . . . . . . . . . . v vii

1. General Introduction . . . . . . . . . . . . . . 1

2. A Discussion of Leptodermous (Thin-skinned) Systems . . . . . 6

2.A. Leptodermous Systems and the Liquid Drop Model. . . . 6

2.B. The Potential Energy of a Leptodermous System . . . . . 8

3. On the Total Energy of Noninteracting Particles in a

Hill-Wheeler Box . . . . . . . . . . . . . . . 18

3.A. Introduction . . . . . . . . . . . . . 18

3.B. The Microscopic Approach . . . . . . . . . . . 19

3.C. The Macroscopic Approach . . . . . . . . . . . 21

3.D. The Meaning of the Approximate Energy Expression . . . . 29

3.E. Results and Comparisons of the Microscopic and

Macroscopic Approaches .............. 36

3.F. Summary and Conclusions .............. . 40

4. On the Symmetric Saddle Point Configurations of a Charged

Conducting Drop . . . . . . . . . . . . . . . 42

4.A. Introduction ................. 42

4.B. Basic Concepts in Fission Theory . . . . . . . . . 43

4.C. Comparison of a Conducting Drop and a Volume

Charged Drop . . . . . . . . . . . . . . 46

4.D. Parameterization of a Conducting Drop . . . . . . . 51

4.E. The Determination of Equilibrium Shapes . . . . . . . 57

H.F. Results .................... 62

4.G. Summary and Conclusions . . . . . . . . . . 65 
5. On the Synthesis of the Liquid Drop Model and the Nilsson Single Particle Model With Applications to the Study of Shape Isomers and the Stability of Superheavy Nuclei . . . . 66

5.A. Introduction .................. 66

5.B. Single Particle Calculations . . . . . . . . . . 67

5.C. The Pairing Force ................ 69

5.D. The Liquid Drop Formula . . . . . . . . . . . . 71

5.E. Generalised Strutinski Prescription and the Synthesis

of the Liquid Drop Model and the Nilsson Model . . . . . 7]

5.E.1. The basis for the synthesis . . . . . . . . 72

5.E.2. Gereralised Strutinski prescription . . . . . 74

5.F. Comparison with Experiments . . . . . . . . . 82

5.F.1. Nuclear masses............. 83

5.F.2. Ground state distortions......... . 85

5.G. Potential Energy Surfaces . . . . . . .... . 87

5.G.1. The structure of spontaneous fission barriers

of heavy and superheavy nuclei... . . . . 87

5.G.2. Spontaneous fission isomers in the actinide region . . . . . . . . . . . . . . . . 91

5.G.3. Shape isomers for the neutron deficient heavy nuclei $(A \sim 206)$. . . . . . . . . . . 93

5.G.4. Uncertainties in the potential energy surfaces. . 95

5.H. Barrier Penetration and Spontaneous Fission Half Lives . . 96

5.I. Stability of Superheavy Nuclei. . . . . . . . . . 100 
5.I.1. Island of stability in the neighbourhood of $\mathrm{Z}=114$ and $\mathrm{N}=184-196 . . . . . . .201$

5.I.2. Possibility of the occurrence of surviving superheavy elements in nature....... . 103

5.J. Possible Experimental Production of Superheavy Nuclei . 106 5.J.l. Heavy ion reactions by available projectiles . . . 106 5.J.2. Heavy ion reactions by future projectiles... . Io 5.J.3. Neutron capture reactions.......... . 110 5.K. Summary . . . . . . . . . . . . . 110 Acknowledgments . . . . . . . . . . . . . . . . . 112 References . . . . . . . . . . . . . . . 113 
ON THE MICROSCOPIC AND MACROSCOPIC ASPECTS

OF NUCLEAR STRUCTURE WITH APPLICATIONS TO

\title{
SUPERHEAVY NUCLEI
}

Chin-Fu Tsang

\author{
Lawrence Radiation Laboratory \\ University of California \\ Berkeley, California
}

May 22, 1969

\section{ABSTRACT}

The thesis is concerned with the relation between a microscopic approach and a macroscopic approach to the study of the nuclear binding energy as a function of neutron number, proton number and nuclear deformations.

First of all we give a general discussion of the potential energy of a system which can be divided into a bulk region and a thin skin layer. We find that this energy can be written down in the usual liquid drop type of expression, i.e., in terms of the volume, the surface area and other macroscopic properties of the system. The discussion is illustrated by a study of noninteracting particles in an orthorhombic potential well with zero potential inside and infinite potential outside. The total energy is calculated both exactiy (a microscopic approach) and also from a 
liquid drop type of expression (a macroscopic approach). It turns out that the lattex approach reproduces the smooth average of the exact results very well.

We next make a digression to study the saddle point shapes of a charged conducting drop on a pure liquid arop model. We compare the properties of a conducting drop with those of a drop whose charges are distributed uniformly throughout its volume. The latter is the usual model employed in the study of nuclear fission. We also determined some of the more important symmetric saddle point shapes.

In the last part of the thesis we generalize a method due to Strutinski to synthesize a microscopic approach (the Nilsson model) and a macroscopic approach (the liquid drop model). The results are applied to realistic nuclei. The possible occurrence of shape isomers comes as a natural consequence of the present calculation. Their trends as a function of neutron and proton members are discussed and the results are tabulated. We also work out the stabilities of the predicted superheavy nuclei, with proton number around 114 and neutron number around 184 and 196. Some of these nuclei appear to have extremely long life times. The possible experimental production of these superheavy nuclei are also discussed. 


\section{General Introduction}

of central importance in the physics of the nucleus is a study of the nuclear binding energy as a function of its deformation and mass number A. Such a study is not only relevant for the ground state masses and deformations, but is also essential in the theory of alpha decay, beta decay as well as the spontaneous fission of the nucleus. It also provides a possible explanation for the so-called shape or fission isomers which have recently induced extensive experimental efforts.

For the last thirty-five years both a microscopic and a macroscopic approach for the calculation of the nuclear binding energy have been developed in parallel. By the macroscopic approach we are thinking of an approach in which one expresses the binding energy as a function of macroscopic properties such as the volume, surface area, and the integrated curvature over the surface of the nucleus. The approach is usually associated with the liquid arop model l) of a nucleus, although in some aspects it is considerably more general than the representation of a nucleus as a fluid droplet. We shall discuss this in detail in the next part of the thesis. By the microscopic approach we are referring to an independent particle model, where one considers the nucleons to move around in an average nuclear field. Residual interactions such as pairing effects can be included. This model is commonly applied with great success to correlate nuclear spectroscopic data and to explain the occurrence of magic numbers. Its successful application to a quantitative description ${ }^{2,3)}$ of nuclear masses and deformabilities is a development of the last few years. 
The microscopic approach is more fundamental than the macroscopic approach in the sense that all results of the latter should be derivable in principle as some sort of an average of the results of the former. Howevex in its present state, it turns out that the independent particle model does not give correctly the absolute values of the experimental binding energies ${ }^{2,3)}$, though it is very successful in reproducing the relative values for neighbouring nuclei. On the other hand the macroscopic approach looks at the nucleus as a whole and considers the binding energy as a sum of the volume, surface, curvature as well as coulomb energy terms. The coefficients in these terms are fitted to experimental values and the approach is able to reproduce the absolute values of the binding energy correctly.

It is important to study the relation between the two approaches and to try to synthesize them in some way so that we may have the useful results of both in a unified approach. The basic idea advocated by Myers and Swiatecki ${ }^{4}$ and Strutinski ${ }^{5)}$ among others is that one should replace the smooth average trends of the results of the independent. particle model, which do not reproduce experimental trends adequately by those from the liquid drop model. The resulting unified model will then represent the real nucleus more closely than is possible with either the microscopic or the macroscopic model.

In the next part of the thesis we will discuss the justification of a macroscopic approach. We are going to look specifically at a 
system with a thin skin (of constant thickness), which we will call a leptodermous ${ }^{\dagger}$ system. A liquid drop is a special example of such a system. By considering just the geometry of this system we can write down its energy as a sum of a volume term, a surface term, and an integrated curvature term.

The third part is a study of the energy of noninteracting nucleons in an orthorhombic potential well with infinite potential walls, which will be referred to as a Hill--Wheeler box ${ }^{6)}$. The total energy as a function of the relative lengths of the sides can be calculated exactly as well as from a macroscopic point of view. A comparison shows that the macroscopic approach does indeed give very closely the smooth trends of the energy calculated exactly.

The fourth part is a study of a pure collective phenomenon. It is a digression from our main theme of studying the relation between the microscopic and macroscopic approaches, to which we shall return in the fifth and last part. It deals with the theory of fission of a charged drop which is electrically conducting so that the charges reside on the surface of the drop. The usual liquid drop model of nuclear fission assumes a charged nonconducting drop with a uniform distribution of charges. However there are sufficient similarities and rather interesting differences to make a study of a charged conducting drop profitable. This is coupled with the great advantage that a macroscopic

t The word "leptos" in Greek means "thin" and the word "derma" means "skin". A leptodermous system is then a system having a thin skin. 
charged conducting liquid drop can actually be investigated experimentally. We have looked at the statics of the fission of such a drop and have been able to determine some of the more important equilibrium shapes of the drop.

The fifth part of the thesis tries to combine the microscopic and macroscopic approaches. Specifically we study the synthesis of the Nilsson model ${ }^{2)}$ and the Iiquid drop model ${ }^{1)}$. Such a unified model is then applied to realistic nuclei from the rare earth elements up to the yet unknown superheavy elements. Besides accounting for many known nuclear properties, we have been able to predict the stabilities of superheavy nuclei and to discuss features in our results which we believe to be associated with the shape or fission isomers. A

+ This part of the thesis was done with the guidance and collaboration of Professor S. G. Nilsson of Lund Institute of Technology, Lund, Sweden. The Nilsson Model calculations ${ }^{3)}$ were developed by $\mathrm{C}$. Gustafson, I. L. Lamm, B. Nilsson, and S. G. Nilsson of Lund Institute of Technology, Sweden. The initial version of the computer problem employing the strutinski Prescription ${ }^{5)}$ was written by J. R. Nix of Los Alamos Scientific Laboratory, University of California.

A. Sobiczewski, Z. Szymanski, and S. Wycech of the Institute of Nuclear Research, Warsaw, Poland, performed the microscopic calculation of the inertial parameter associated with spontaneous fission 7 ) that is used in the present work. 
discussion is also given on the prospect of the experimental production
of superheavy elements as well as on the possibility that they might
occur in nature.
Table l. The various parts of the thesis are presented schematically in

discussion is also given on the prospect of the experimental production
of superheavy elements as well as on the possibility that they might
occur in nature.
Table l. The various parts of the thesis are presented schematically in 
2. A Discussion of Leptodermous (Thin-skinned) Systems

\section{A. Leptodermous Systems and the Liquid Drop Model}

In this part of the thesis we shall discuss the nature of the potential energy expression for a class of physical systems that may be considered as consisting of a bulk region and a thin surface region. (We shall refer to such systems as leptodermous.) In some cases, when the bulk region is uniform, the potential energy expression reduces to that usually associated with the Liquid Drop Model of a nucleus. The principal energy terms are then a volume energy and a surface energy. For historical reasons, however, the Iiquid Drop Model of the nucleus is often understood to imply more than just the presence of a bulk region and a surface region. Thus it is often taken to imply the existence of strong correlations between the particles constituting the system, and, in dynamical problems, it is frequently taken to be synonymous with the assumption of an irrotational flow of fluid. When understood in this sense the Liquid Drop Model is an extremely poor representation of the nucleus. This seems to have led to an unjustified skepticism as regards the relevance of the Liquid Drop Model for the description of even the purely static aspects of the nuclear binding energies and deformabilities.

To clarify this confusion we would like to stress two points. First, the validity of the Liauid Drop type of expressions for the description of static properties has of course nothing to do with further possible assumptions concerning dynamics, such as the assumption 
of irrotational flow. Secondly, as we hope to demonstrate, it has also nothing to do with the assumption of strong correlations between the particles constituting the system. In fact the basic condition for the validity of a Licuid Drop type of expression for the potential energy is the possibility of dividing the system into a bulk region and a thin surface region. We have thought it worthwhile to introduce a name leptodermous - to describe systems satisfying this specific assumption regarding their constitution, in order to avoid confusion with the less well defined phrase "Liquid Drop Model".

Examples of leptodermous systems are

1) A drop of water (made up of strongly interacting molecules).

2) A classical gas of noninteracting point particles in a container.

3) A degenerate gas of noninteracting fermions in an external. potential well.

4) A system of particles interacting by short-ranged saturating: forces treated in the statistical Thomas-Fermi approximation ${ }^{8)}$.

5) Same as (4), but with nonsaturating electrostatic forces also present ${ }^{8}$.

6) Amorphous solids.

7) Nuclei.

In example 1, the constituent particles interact strongly and are highly correlated. Example 2 is a trivial special case of noninteracting particles where the skin thickness is zero. Example 3, which is the subject of Part 3 of this thesis, is a case of noninteracting 
particles treated quantum mechanically and is a prototype of a nuclear shell model. The skin thickness turns out to be of the order of the wavelength of the fastest particle present. In example 4 there are (saturating) forces between the particles but no correlations and the quantum nature of individual particles is disregarded. The skin thickness turn's out to be of the order of the range of the forces between the particles. In example 5 the presence of electrostatic forces results in a nonuniform bulk density but the thickness of the surface region remains as in 4. In the case of an amorphous solid (example 6) the potential energy would, we presume, also be a sum of a volume and a surface term provided any deformations of the system were sufficiently slow so that internal stresses would be relievedby plastic flow. Example 7, a nucleus, is known from electron scattering experiments 9$)$ to satisfy moderately well the condition of being thin-skinned. The nuclear potential energy also appears to be well represented by a bulk term and a surface term.

Examples of nonleptodermous systems are atoms and stars, for which it is not possible to make a distinction between a bulk region and a thin surface region.

2.B. The Potential Energy of a Leptodermous System

Let us write down the potential energy of a leptodermous system with a uniform bulk particle density ${ }^{\dagger} \rho_{c}$. The density $\rho$ falls to

+ The more general case where the bulk density is smoothly varying (Example 5 above) can be treated as a straightforward generalisation ${ }^{8)}$ of the present calculation. 
zero in a thin surface layer. The total number of particles is given by

$$
\mathbb{N}=\int \rho d \tau
$$

The total energy is

$$
E=\int \rho \text { e d } \tau \text {, }
$$

where $e$ is the energy per particle at every point. In general e is a functional of the density distribution $\rho$. It is the purpose of the present section to write down the potential energy $E$ as a sum of terms proportional to the volume, surface area, and integrated curvature of the system.

Let us assume that in the bulk region every point is just like any other point in the sense that a constant value $e_{c}$ can be written for the energy per particle in the bulk. Then Eg. (1) may be written

$$
E=\int \rho e_{c} d \tau+\int \rho\left(e-e_{c}\right) d \tau \text {. }
$$

Let" us define an "equivalent system" as one with the same bulk density ${ }_{c}$ but having a sharp surface; the original leptodermous system results when the sharp surface is diffused into a skin layer of constant thickness. Thus the volume of the equivalent system is

$$
V=\frac{\int \rho d \tau}{\rho_{c}} .
$$

Hence we may write

$$
E=e_{c} \rho_{c} V+\int \rho\left(e-e_{c}\right) d \tau .
$$


The integrand in the second term is nonzero only in the thin skin layer because $\rho$ tends to zero outside the skin and $e-e_{c}$ inside. Now we define a normal $n$ to any point in the surface with $n=0$ at the sharp surface of the equivalent system. Then the integrand as a function of $\mathrm{n}$ is zero for large positive or negative values of $\mathrm{n}$. We may denote this integrand by

$$
F(n, \kappa) \equiv \rho\left(e-e_{c}\right),
$$

where we have indicated that the integrand is also a function of the curvature $\kappa$ at the point on the surface ${ }^{\dagger}$. Let us write down an

+ In general one would think that $F$ is a function of $1 / R_{1}$ and $1 / R_{2}$ where $R_{1}$ and $R_{2}$ are the principal radii of curvature in two perpendicular planes through the point. If one makes an expansion about a plane, for which $1 / R_{1}=0=1 / R_{2}$, then $F\left(\frac{1}{R_{1}}, \frac{1}{R_{2}}\right)=F(0,0)+\frac{1}{R_{1}}\left(\frac{\partial F}{\partial \frac{1}{R_{1}}}\right)_{0}+\frac{1}{R_{2}}\left(\frac{\partial F}{\partial \frac{1}{R_{2}}}\right)_{0}+\cdots \cdot$

Since all directions in a plane are equivalent, the two derivatives of $F$ with respect to $1 / R_{1}$ and $I / R_{2}$ are equal.

$$
F\left(\frac{1}{R_{1}}, \frac{1}{R_{2}}\right)=F(0,0)+\left(\frac{1}{R_{1}}+\frac{1}{R_{2}}\right)\left(\frac{\partial F}{\partial \frac{1}{R_{1}}}\right)_{0}+\cdots \cdot
$$

Hence to the first order in the deviations from a plane we have, $F(\kappa)=F(0)+\kappa\left(\frac{\partial F}{\partial \kappa}\right)_{0}$ where $\kappa=\frac{1}{R_{1}}+\frac{1}{R_{2}}, F$ is then a function of $k$ rather than of the separate components $1 / R_{1}$ and $1 / R_{2}$. 
expansion of $F$ in $\kappa$ to the first order:

$$
F(n, \kappa)=F(n, 0)+\kappa F^{\prime}(n, 0),
$$

where the prime indicates differentiation with respect to $\kappa$. We also write the volume element $d \tau$ in terms of the co-ordinate, $n$, and the curvature, $k$, to the first order,

$$
d \tau=(1+\kappa n) d n d \sigma,
$$

where do is the area element at the point on the equivalent sharp surface. Hence we have

$$
\begin{aligned}
& \mathrm{E}=\epsilon_{c} \rho_{C} \mathrm{~V}+\int d \sigma \int \mathrm{dn}(1+\kappa n)\left[\mathrm{F}(\mathrm{n}, 0)+\kappa \mathrm{F}^{\prime}(\mathrm{n}, 0)\right] \\
& =e_{c} \rho_{c} \mathrm{~V}+\int d \sigma \int d n \mathrm{~F}(\mathrm{n}, 0)+\int \mathrm{d} \sigma \kappa \int \mathrm{dn}\left[\mathrm{nF}(\mathrm{n}, 0)+\mathrm{F}^{\prime}(\mathrm{n}, 0)\right] \ldots
\end{aligned}
$$

Since $F(n, 0)$ and $F^{\prime}(n, 0)$ are evaluated for $\kappa=0$, i.e., for a plane surface, they are independent of the position on the surface and the surface integrations in the second and third terms may be carried out at once. If we define the surface area $S$ and the integrated curvature $I$ of the equivalent sharp surface by

$$
\begin{aligned}
& S=\int d_{\sigma} ; \\
& L=\int \kappa d \sigma,
\end{aligned}
$$

we have the result

$$
\mathrm{E}=\mathrm{aV}+\mathrm{bS}+\mathrm{cL}+\cdots,
$$


where

$$
\begin{aligned}
& \mathrm{a}=e_{\mathrm{c}} \rho_{\mathrm{c}} \\
& \mathrm{b}=\int \mathrm{dn} \mathrm{F}(\mathrm{n}, 0) \\
& \mathrm{c}=\int \operatorname{dn}\left[\mathrm{F}^{\prime}(\mathrm{n}, 0)+\mathrm{nF}(\mathrm{n}, 0)\right] .
\end{aligned}
$$

Equation (2) shows how the energy of a leptodermous system may be decomposed, under the stated assumptions, into volume, surface and curvature terms. Equations (3) shows explicitly how the relevant coefficients can be calculated from the properties of the system. The coefficient $a$ is the volume energy density. The coefficient $b$ is the surface tension coefficient which gives the difference per unit area of a plane surface, between the energy of a number of particles touching the surface and the energy of the same number of particles in the bulk.

The coefficient $\mathrm{c}$ is the curvature coefficient which describes the modification in the effective surface energy resulting from the curvature of the surface. Note that both $b$ and $c$ are integrals over functions localized in the surface layer and may therefore be regarded. as intrinsic properties of the surface region. As discussed in Ref. 8 (pp. 69 and 126) the coefficient $c$ consists of two parts. The first part is associated with the modified conditions (i.e., increased exposure or "fewer neighbours") for particles in a curved surface. The modification is expressed in terms of $F^{\prime}$ describing the response of the surface energy function $F$ to a bending of the surface. The second 
part is associated with the purely geometrical fact that a given surface layer contains fewer particles when (convexly) curved than when flat. (The two effects are usually of opposite sign and may even cancel exactly. See Ref. 8)

It should be noted that the simple structure of $\mathrm{Eg}$. (2) and the above interpretation of the coefficients of $S$ and $L$ is intimately related to our definition of an equivalent sharp volume $V$ (and the associated area $S$ and integrated curvature $L$ ) of the originally diffused leptodermous system. The fact that for a system with a diffused surface there appears at first sight to be a degree of arbitrariness in the definition of its volume, surface area, and integrated curvature, has led in the past to some confusion and even to serious misinterpretations of the surface tension coefficient ${ }^{6)}$.

We denote the remainder of this section to a discussion of these problems. We shall give below a detailed demonstration of the sometimes subtle effects involved, but we would like to state at the outset what the root of the problem is: if the volume, surface area, etc. of the diffuse system is defined in any other way than the above (i.e., by means of the equivalent system with a sharp surface which contains the total number of particles at the bulk density) then in general the assocjated volume energy differs from the true bulk energy by terms which may have the appearance of surface and curvature terms (even though their origin is in the bulk). The result is in fact a host of spurious terms parading as contributions to the surface tension and curvature correction coefficients and creating confusion in the identification of correct values of these coefficients. 
To illustrate this let us consider what would happen to Eq. (2) if instead of the equivalent sharp volume $V$ (and its area and integrated curvature) we choose to express the energy in terms of another volume $\Omega$ and its area $\Sigma$ and the integrated curvature $\Lambda$. The cases of most relevance and which have caused confusion in the past are those in which $\Omega$ is related to $V$ by a (small) normal shift of the surface by an amount $t$, say, of the order of the diffuseness of the surface. The relations between $\mathrm{V}, \mathrm{S}, \mathrm{L}$ and $\Omega, \Sigma, \Lambda$ are easily derived by noting that the element of area on a normally displaced surface is related to an element of area on the original surface by

$$
d \sigma_{\text {displaced }}=(1+\kappa t) d \sigma .
$$

Hence

$$
\Sigma=\oint(1+\kappa t) d \sigma=s+t L .
$$

Also

$$
\begin{aligned}
\Omega & =V+\int_{t=0}^{t} d \cdot \oint(1+\kappa t) d \sigma \\
& =V+S t+\frac{1}{2} t^{2} I .
\end{aligned}
$$

Inverting these relations we may write

$$
\begin{aligned}
& V=\Omega-\Sigma t+\frac{1}{2} t^{2} \Lambda+\cdots \\
& S=\Sigma-t \Lambda+\cdots \\
& L=\Lambda+\cdots .
\end{aligned}
$$


(For our purposes, it suffices to write the three relations to successively lower orders in t.) We may now insert the above relations in Eq. (2), which may first be rewritten as

$$
E=e(\rho) \mathbb{N}+b(\rho) S+c(\rho) L
$$

We have displayed explicitly the dependence of the coefficients on the bulk density $\rho$. (We have dropped the suffix $c$ on $\rho$ as well as e, the bulk energy per particle. We have also written the leading term as eN instead of aV.) our objective is to write $\mathrm{E}$ as a function of $\Omega, \Sigma$, and $\Lambda$. The density $\rho$ is given by $N / V$, which is related to $\tilde{\rho} \equiv N / \Omega$ through $\mathrm{Eq} \cdot(4)$. Thus

$$
\begin{aligned}
\rho=\frac{N}{V} & =\frac{N}{\Omega}\left(1-\frac{\sum}{\Omega} t+\frac{1}{2} t^{2} \frac{\Lambda}{\Omega}\right)^{-1} \\
& =\tilde{\rho}\left[1+\frac{\Sigma}{\Omega} t-\frac{1}{2} \frac{\Lambda}{\Omega} t^{2}+\left(\frac{\sum}{\Omega}\right)^{2} t^{2}+\cdots\right] .
\end{aligned}
$$

If we insert his expression for $\rho$ in the argument of $e(\rho)$, say, and make a Taylor expansion about the value $\tilde{e} \equiv e(\tilde{\rho})$, we find

$$
\epsilon(\rho)=\tilde{e}+e^{\prime} \frac{\Sigma}{\Omega} t+\left[\left(e^{\prime}+\frac{1}{2} e^{\prime \prime}\right)\left(\frac{\Sigma}{\Omega}\right)^{2}-\frac{1}{2}\left(\frac{\Lambda}{\Omega}\right) e^{\prime}\right] t^{2}
$$

where

$$
\begin{aligned}
& e^{\prime}=\left(\rho \frac{\partial e}{\partial \rho}\right)_{\tilde{\rho}} \\
& e^{\prime \prime}=\left(\rho^{2} \frac{\partial^{2} e}{\partial \rho^{2}}\right)_{\tilde{\rho}} .
\end{aligned}
$$


Dealing similarly with $b(\rho)$ and $c(\rho)$ and using Eqs. (4), we find

$$
E=\tilde{e N}+\tilde{b} \Sigma+\tilde{c} \Lambda+\tilde{d}\left(\Sigma^{2} / \Omega\right)
$$

where

$$
\begin{aligned}
& \tilde{b}=b+e^{\prime} \tilde{\rho} t \\
& \tilde{c}=c-b t-\frac{1}{2} e^{\prime} \tilde{\rho} t^{2} \\
& \tilde{d}=b^{\prime} t+\left(e^{\prime}+\frac{1}{2} e^{\prime \prime}\right) \tilde{\rho} t^{2} .
\end{aligned}
$$

Suppose now we assume the volume $\Omega$ to be proportional to $N$ and independent of the shape of the system (i.e., we take $\tilde{\rho}$ to be constant). The coefficients $\tilde{e}, \tilde{a}, \tilde{b}, \tilde{c}, \widetilde{d}$ are then constants and Eq. (5) gives the total energy as a function of $N($ or $\Omega), \Sigma$, and. $\Lambda$, i.e., as a function of volume, area, and integrated curvature of a surface obtained from the standard equivalent sharp surface by a small normal shift $t$.

To the relevant order this equation is equivalent to Eq. (2), but note the following features. First, in addition to terms proportional to $N$ (or $\Omega$ ), $\Sigma$, and $\Lambda$, there is a new term proportional to $\Sigma^{2} / \Omega$. Second, the values of the coefficients of $\Sigma$ and $\Lambda$ are different from the previous values and if one were to identify $\tilde{b}$ with the surface tension coefficient and $\tilde{c}$ with the curvature correction coefficient one would deduce values quite different from those given by Eq. (2). Thus the coefficient of $\Sigma$ has an additional term $e^{\prime} \tilde{\rho} t$ which comes from the bulk energy and the coefficient of $\Lambda$ has two additional terms, 
-bt, which comes from the surface energy, and $\frac{l}{2} e^{\prime} \tilde{\rho} t^{2}$, which comes from the bulk energy. In Eq. (5) there are altogether six spurious terms e $e^{\tilde{\rho} t \Sigma}, \quad-b t \Lambda, \quad-\frac{1}{2} e^{\prime} \tilde{\rho} t^{2} \Lambda, \quad b \cdot t \Sigma^{2} / \Omega, \quad e^{\prime} \tilde{\rho} t^{2} \Sigma^{2} / \Omega$, and $\frac{1}{2} e^{\prime} \rho t^{2} \Sigma^{2} / \Omega$. Note that of these, the first, third, and fifth vanish if $e^{\prime}=0$ (i.e., if $\left.(\rho \mathrm{de} / \partial \rho)_{\tilde{\rho}}=0\right)$. This means that for a system whose bulk energy is stationary with respect to density deviations from $\tilde{\rho}$ (i.e., a saturating system) these terms do not appear. It has recently been shown $^{8)}$ that for a saturating system the surface tension coefficient is also stationary, i.e., $\mathrm{b}^{\prime}=0$, and the fourth term would also be absent for such a system. The second and sixth terms are, however, present even for a saturating system. For a nonsaturating system (like a Fermi gas or a nuclear individual particle model in an external potential well) all six terms are present, and great care must be exercised in interpreting the results of the energy calculations of such systems, unless the proper choice of the equivalent sharp surface has been made to begin with. 
3. On the Total Energy of Noninteracting Particles in a Hill-Wheeler Box

3.A. Introduction

It is the purpose of this part of the thesis to demonstrate the relation between a microscopjc approach and a macroscopic approach on as simple a model as possible. One such model is furnished by noninteracting spinless Fermi-Dirac particles in an orthorhombic box with infinite repulsive potential outside and zero potential inside. Such a box will be referred to as a Hill-Wheeler box. It was first introduced by Hill and wheeler ${ }^{6}$ who applied it in an attempt to obtain the coefficients of the nuclear surface and curvature energies. Due to a misinterpretation of their equations they did not get the correct results which had been given by swiateckilo) in a semi-infinite model. The correct interpretation for the surface energy was given by Knaak et alll). $\mathrm{Hilf}^{12}$ ) considered also the cases of cylindrical and spherical boxes.

The Hill-Wheelex box is a particularly simple model because all the wavefunctions in the box can be easily written down in terms of trigonometric functions. The total energy as a function of the particle number and the deformation of the box can be exactly written down. On the other hand we can also take a macroscopic point of view and approximate the total energy by a function of macroscopic quantities such as the volume and surface area of the Hill wheeler box. We shall demonstrate that this macroscopic approach gives the smooth trends of the exact results very well. 


\section{B. The Microscopic Approach}

The solution of the problem of noninteracting spinless FermiDirac particles in an orthorhombic infinite potential well is wellknown. It suffices that we indicate the main results below.

Let the three sides of the box be specified by

$$
\begin{aligned}
& \mathrm{a}=\mathrm{R} \exp \left[\alpha \cos \left(\gamma-\frac{2 \pi}{3}\right)\right] \\
& \mathrm{b}=\mathrm{R} \exp \left[\alpha \cos \left(\gamma+\frac{2 \pi}{3}\right)\right] \\
& \mathrm{c}=\mathrm{R} \exp [\alpha \cos \gamma]
\end{aligned}
$$

where $\alpha$ and $r$ are two deformation parameters. We have chosen the definition such that the volume of the box is equal to $\mathrm{R}^{3}$ independent of $\alpha$ and. $r$. When $\alpha=0$ and $\gamma=0$, the box is simple cubic. When $r=0$ and $\alpha>0$, we have $a=b<c$ and the box is "prolate". When $r=\pi / 3$ and $\alpha>0$, we have $a=c>b$ and the box is "oblate".

All the wave functions in the box have to go to zero at the walls. This requires the single particle energy levels to be given by

$$
\epsilon=\pi^{2}\left(\frac{n^{2}}{a^{2}}+\frac{m^{2}}{b^{2}}+\frac{e^{2}}{c^{2}}\right) \frac{\hbar^{2}}{2 m}
$$

where $M$ is the mass of the particle. The quantum numbers $n, m$, and $\ell$ are integers greater than or equal to one. The single particle energies as a function of the deformation parameter $\alpha$ (with $r=0$ ) are shown in Fig. I, which is essentially the familiar Nilsson diagram ${ }^{2}$ ) 
for the present case of spinless fermions in a Hill-Wheeler box. The above calculation is in effect a prototype of the Nilsson model

calculations $^{2)}$. We have indicated in the figure the positions at $\alpha=0$, where large gaps among the levels are found. These correspond to magic numbers where special binding occurs. For a particular shape of the box, a given number of particles fills the energy levels up to a level whose energy is referred to as the Fermi energy $\epsilon_{F}$. All levels below $\epsilon_{F}$ are filled and all levels above $\epsilon_{F}$ are empty.

We can associate each particle with a momentum vector $\mathrm{k}_{\mathrm{i}}$ such that the particle's energy is given by

$$
\frac{\hbar^{2}}{2 M}\left|k_{i}\right|^{2}=\epsilon_{i}
$$

Let us look at the momentum space with the coordinate axes along the three axes of the Hill-Wheeler box. Then an octant of a sphere is drawn with its center at the origin and having positive values of $k_{x}$, $k_{y}$, and $k_{z}$. The radius of the octant $k_{F}$ is given by

$$
\frac{\hbar^{2}}{2 M}\left|k_{F}\right|^{2}=\epsilon_{F}
$$

A lattice is constructed in this space by choosing the units in $k_{x}$, $\mathrm{k}_{\mathrm{y}}$, and $\mathrm{k}_{\mathrm{z}}$ directions to be $\pi / \mathrm{a}, \pi / \mathrm{b}$, and $\pi / \mathrm{c}$ respectively. The designation of the lattice points in $\left(k_{x}, k_{y}, k_{z}\right)$ would be just the set of quantum numbers $n, m, l$ of the energy levels. In particular the lattice point $(1,1 ; 1)$ corresponds to the lowest energy state with $\mathrm{n}=1, \mathrm{n}=1$ and $\ell=1$. Since the particles fill the energy levels up 
to $\epsilon_{F}$, all the lattice points in the momentum space within the octant of radius $k_{F}$ are occupied by particles, and those without are not. The number of lattice points inside the octant is equal to the number of particles in the box. The energy of the system, $E$, is given by

$$
E=\sum_{i}\left|\underset{\sim i}{k_{i}}\right|^{2} \frac{\hbar^{2}}{2 M},
$$

where the summation is carried over all the lattice points inside the octant.

\section{C. The Macroscopic Approach}

By the macroscopic approach we hope to write down the total energy of the particles in a Hill-Wheeler box in terms of some macroscopic quantities. One way to do this is to imagine each lattice point in $\underset{\sim}{\mathrm{k}}$ space inside the octant of radius $\mathrm{k}_{\mathrm{F}}$ to be smeared out into an orthorhombic box centered at the lattice point and with sides equal to $\pi / a, \pi / b$, and $\pi / c$. Such orthorhombic boxes build up into an octant with slabs of thickness $\mathrm{w}_{1} \equiv \pi / 2 \mathrm{a}, \mathrm{w}_{2} \equiv \pi / 2 \mathrm{~b}$, and $\mathrm{w}_{3} \equiv \pi / 2 \mathrm{c}$ cut away from the planes $k_{x}=0, k_{y}=0$, and $k_{z}=0$ respectively. (See Fig. 2, which shows only the $k_{x} k_{y}$ plane.) Also on the curved surface of the octant, bumps and dips occur that correspond to parts of the boxes sticking out and parts missing from the smooth curved surface. We can adjust the position of this curved surface to an effective Fermi radius $q$ (see Fig. 2) such that the volumes of the bumps and dips cancel. The volume $F$ of the resulting "incomplete octant" with slabs 
cut away is then related to $\mathbb{N}$, the number of particles in the HillWheeler box, by

$$
F=\frac{\pi^{3}}{a b c} N=8 w_{1} w_{2} w_{3} N
$$

since the unit box around each lattice point has the volume

$$
v=\frac{\pi^{3}}{a b c}
$$

A straightforward calculation gives $F$ as a function of $q$ :

$$
\begin{aligned}
& F= \frac{1}{3} \frac{\pi}{6} q^{3}-\frac{1}{3} q^{3} \tan ^{-1} \frac{w_{1} w_{2}}{q\left(q^{2}-w_{1}^{2}-w_{2}^{2}\right)^{\frac{1}{2}}} \\
&+\left(\frac{1}{2} q^{2} w_{1}-\frac{1}{6} w_{1}^{3}\right)\left(\sin ^{-1} \frac{w_{2}}{\left(q^{2}-w_{1}^{2}\right)^{\frac{1}{2}}}+\sin ^{-1} \frac{w_{3}}{\left(q^{2}-w_{1}^{2}\right)^{\frac{1}{2}}}\right) \\
&-\frac{\pi}{4} q^{2} w_{1}+\frac{1}{3} w_{1} w_{2}\left(q^{2}-w_{1}^{2}-w_{2}^{2}\right)^{\frac{1}{2}}+\frac{\pi}{12} w_{1}^{3} \\
&-\frac{1}{3} w_{1} w_{2} w_{3}+\text { Permutations with respect to } w_{1}, w_{2}, w_{3} \\
& \text { (including the first term) }
\end{aligned}
$$

If we assume $w_{1}, w_{2}, w_{3} \ll q$, which is the case corresponding to a large number of particles, we can make an expansion in $\mathrm{w}_{1} / \mathrm{q}, \mathrm{w}_{2} / \mathrm{q}$, and $w_{3} / q$ 


$$
F=\frac{\pi}{6} q^{3}-\frac{\pi}{4} q^{2}\left(w_{1}+w_{2}+w_{3}\right)+q\left(w_{1} w_{2}+w_{2} w_{3}+w_{3} w_{1}\right)
$$

The first three terms are equivalent to the results obtained by Hill and Wheeler ${ }^{6)}$. The first term corresponds to the volume of the whole octant in $\mathrm{k}$ space. The second term corresponds to the slabs that are to be cut away from the octant at the $\mathrm{k}_{\mathrm{x}}=0, \mathrm{k}_{\mathrm{y}}=0$, and $\mathrm{k}_{\mathrm{z}}=0$ planes. Where the slabs intersect we subtract too much by the columns along the $\mathrm{k}_{\mathrm{x}}, \mathrm{k}_{\mathrm{y}}$, and $\mathrm{k}_{\mathrm{z}}$ axes. This is the origin of the third term. The fourth and fifth terms represent even higher corrections to the geometry of the volume $\mathrm{F}$ of the incomplete octant. Given $\mathrm{F}$, one can calculate the particle number $N$. Conversely if we are given $N$, we can find the effective Fermi momentum $q$.

We next proceed to calculate the energy in terms of macroscopic quantities. The exact energy $\mathrm{E}$ calculated in the last section is given by $\mathrm{Eq}$. (6), which is a summation of the function $k_{i}^{2} x^{2} / 2 \mathrm{M}$ over the lattice points. For the sake of clarity, we make two provisional simplifications. First we assume each lattice point to be smeared out into an orthorhombic box around this point with sides $\pi / a, \pi / b$, and $\pi / c$. Thus instead of $k_{i}^{2}$ in the equation, we use an integral of $k^{2}$ over the box. Second, near the Fermi surface we smooth out the bumps and dips by means of a smooth curved surface of radius $q$ as defined before. In other words we replace the integration of $k^{2}$ over a bump by that over a dip of equal volume. We shall discuss the consequences of these 
two simplifications in the next section. What these simplifications amount to is that one can now get an approximate energy $E^{\prime}$ by integrating $\hbar^{2} \mathrm{k}^{2} / 2 \mathrm{M}$ over the volume $\mathrm{F}$

$$
E^{\prime}=\frac{\hbar^{2}}{2 M} \frac{a b c}{3} I \text {, }
$$

where

$$
\begin{aligned}
& I=\frac{1}{15} q^{5} \\
& x\left(\frac{\pi}{2}-\tan ^{-1} \frac{w_{1} w_{2}}{q\left(q^{2}-w_{1}^{2}-w_{2}^{2}\right)^{\frac{1}{2}}}-\tan ^{-1} \frac{w_{2} w_{3}}{q\left(q^{2}-w_{2}^{2}-w_{3}^{2}\right)^{\frac{1}{2}}}\right. \\
& \left.-\tan ^{-1} \frac{w_{3} w_{1}}{q\left(q^{2}-w_{3}^{2}-w_{1}^{2}\right)^{\frac{1}{2}}}\right)-\frac{\pi}{16} q^{4}\left(w_{2}+w_{3}\right) \\
& +\left(\frac{1}{8} q^{4} w_{3}-\frac{1}{12} q^{2} w_{3}^{3}+\frac{1}{40} w_{3}^{5}\right)\left(\sin ^{-1} \frac{w_{2}}{\left(q^{2}-w_{3}^{2}\right)^{\frac{1}{2}}}+\sin ^{-1} \frac{w_{1}}{\left(q^{2}-w_{3}^{2}\right)^{\frac{1}{2}}}\right) \\
& +\left(\frac{1}{8} q^{4} w_{2}-\frac{1}{12} q^{2} w_{2}^{3}+\frac{1}{40} w_{2}^{5}\right)\left(\sin ^{-1} \frac{w_{3}}{\left(q^{2}-w_{2}^{2}\right)^{\frac{1}{2}}}+\sin ^{-1} \frac{w_{1}}{\left(q^{2}-w_{2}^{2}\right)^{\frac{1}{3}}}\right) \\
& +\left(\frac{1}{6} q^{2} w_{1}^{3}-\frac{1}{10} w_{1}^{5}\right)\left(\sin ^{-1} \frac{w_{2}}{\left(q^{2}-w_{1}^{2}\right)^{\frac{1}{2}}}+\sin ^{-1} \frac{w_{3}}{\left(q^{2}-w_{1}^{2}\right)^{\frac{1}{2}}}\right) \\
& +\left(q^{2}-w_{1}^{2}-w_{2}^{2}\right)^{\frac{1}{2}}\left(-\frac{7}{120} q^{2} w_{1} w_{2}+\frac{3}{20} w_{1}^{3} w_{2}+\frac{1}{40} w_{1} w_{2}^{3}\right)
\end{aligned}
$$

(Equation continued on next page) 
(Equation continued)

$$
\begin{aligned}
& +\left(q^{2}-w_{3}^{2}-w_{1}^{2}\right)^{\frac{1}{2}}\left(-\frac{7}{120} q^{2} w_{3} w_{1}+\frac{3}{20} w_{3} w_{1}^{3}+\frac{1}{40} w_{3}^{3} w_{1}\right) \\
& +\left(q^{2}-w_{2}^{2}-w_{3}^{2}\right)^{\frac{1}{2}}\left(\frac{3}{20} q^{2} w_{2} w_{3}-\frac{7}{120} w_{2}^{3} w_{3}-\frac{7}{120} w_{2} w_{3}^{3}\right) \\
& +\frac{\pi}{24} q^{2}\left(-2 w_{1}^{3}+w_{2}^{3}+w_{3}^{3}\right)+\frac{\pi}{80}\left(4 w_{1}^{5}-w_{2}^{5}-w_{3}^{5}\right)-\frac{1}{3} w_{1}^{3} w_{2} w_{3} \\
& + \text { Permutations with respect to } w_{1}, w_{2}, w_{3} .
\end{aligned}
$$

If we again make an expansion in $w_{1} / q, w_{2} / q$, and $w_{3} / q$, we get

$$
I=\frac{\pi}{10} q^{5}-\frac{\pi}{8} q^{4}\left(w_{1}+w_{2}+w_{3}\right)+\frac{1}{3} q^{3}\left(w_{1} w_{2}+w_{2} w_{3}+w_{3} w_{1}\right)+\cdots \cdot
$$

We can express the energy $E^{\prime}$ in terms of $\mathbb{N}$ by substituting for $q$. Remembering that $\Omega=a b c$, we get after some algebra,

$$
\begin{aligned}
& \frac{2 M}{\hbar^{2}} E^{\prime}=\frac{3}{5}\left(6 \pi^{2}\right)^{\frac{2}{3}}\left(\frac{N}{\Omega}\right)^{\frac{2}{3}} N+\frac{3 \pi}{16}\left(6 \pi^{2}\right)^{\frac{2}{3}} \frac{\sum}{\Omega^{2 / 3}}\left(\frac{N}{\Omega}\right)^{\frac{2}{3}} N^{\frac{2}{3}} \\
& +\left[\frac{3 \pi^{2}}{64} \frac{\Sigma^{2}}{\Omega^{4 / 3}}-\frac{1}{2} \frac{\Lambda}{\Omega^{1 / 3}}\right]\left(\frac{N}{\Omega}\right)^{\frac{2}{3}} N^{\frac{1}{3}}+\cdots
\end{aligned}
$$


wheret

$$
\begin{aligned}
& \Omega=a b c \\
& \Sigma=2(a b+b c+c a) \\
& \Lambda=\frac{\pi}{2} 4(a+b+c) .
\end{aligned}
$$

If we assume that $\mathbb{N}$ is proportional to $\Omega$, we find that the terms in the energy are proportional to $N, N^{2 / 3}$, and $N^{1 / 3}$. We shall refer to these terms as the $N$-term, $N^{2 / 3}$-term, and $N^{1 / 3}$-term respectively. To bring out the shape dependence, we may make a simple rearrangement in the equation, giving

$\dagger$ It is found that $\Lambda$ is just the integrated curvature of a HillWheeler box. The plane surfaces of the box have zero curvature. At the edges of the box, we have an infinitely large curvature on an infinitesimal surface area. The integrated curvature may be calculated by first rounding off the edge and then taking the appropriate limit of the integrated curvature of this rounded edge. In general at the edge formed by two plane surfaces at an angle $\alpha$, the integrated curvature turns out to be just $\alpha$ per unit length. For the HillWheeler box, the integrated curvature is thus $\pi / 2$ per unit edge length. 


$$
\begin{aligned}
\frac{2 M}{\hbar^{2}} E^{\prime} & =\frac{3}{5}\left(6 \pi^{2}\right)^{\frac{2}{3}}\left(\frac{N}{\Omega}\right)^{\frac{5}{3}} \Omega+\frac{3 \pi}{16}\left(6 \pi^{2}\right)^{\frac{2}{3}}\left(\frac{N}{\Omega}\right)^{\frac{4}{3}} \Sigma \\
+ & \frac{N}{\Omega}\left[\frac{3 \pi^{2}}{64} \frac{\Sigma^{2}}{\Omega}-\frac{1}{2} \Lambda\right]+\cdots
\end{aligned}
$$

This equation has the same form as Eq. (5) in Part II of this thesis, where we discussed the potential energy of a leptodermous system. The system of particles in a Hill-Wheeler box is in fact an example of a leptodermous system. Swiatecki ${ }^{10}$ ) has shown that its skin thickness is of the order of the wavelength of the fastest particle in the box. More specifically, with reference to the volume $\mathrm{V}$, surface area $\mathrm{S}$, and integrated curvature $\mathrm{L}$ of an "equivalent system" (with zero skin thickness, containing the same total number of particles at the bulk density), one may express the volume $\Omega$ and area $\Sigma$ of the Hill-Wheeler box to the first order as follows:

$$
\begin{aligned}
& \Omega=V+t_{S}+\cdots \\
& \Sigma=S+\cdots
\end{aligned}
$$

where $t$ is the skin thickness (Swiatecki ${ }^{10}$ ) used the symbol b) given by $^{10)}$

$$
t=\frac{3 \pi}{8} \frac{1}{k_{F}}=\frac{3 \pi}{8}\left(\frac{v}{6 \pi}\right)^{\frac{1}{3}} .
$$

This is to be compared with a characteristic dimension of the box given by $\mathrm{v}^{1 / 3}$. Thus the skin thickness is smaller by one order in $N^{1 / 3}$. Hence particles in the Hill-Wheeler box form a leptodermous system. 
Let us write the energy Eq. (9) in terms of the V, $\mathrm{S}$, and $L$ of the equivalent system, which is the reference system we should use, as explained in Part II. Then one has to write Eqs. (10) up to the curvature term (see Eq. (4)). Here a difficulty appears, associated with the singular nature of the boundaries of a Hill-Wheeler box (the occurrence of infinite curvatures over infinitesimal areas of the boundary). For a smooth leptodermous system the additional terms can be found by expanding about a plane surface (see p. 14). The result is two extra terms: $\frac{1}{2} t^{2} \Lambda$ in the expression for $V$ and $-t \Lambda$ in the expression for S. (see Eq. 4) These terms are thus both determined once $t$ is known. It turns out that for a Hill-Wheeler box the additional terms are still proportional to $\Lambda$ but the constants of proportionality are not, in general, $\frac{1}{2} t^{2}$ and $t$. They are unknown coefficients which could only be determined from a closer study of the properties of a Fermi gas in the neighbourhood of a right-angled edge in a potential well. In considering the relations between $\Omega, \Sigma, \Lambda$ and $V, S, L$ we are thus force to write

$$
\begin{aligned}
& \Omega=V+t S+g L+\cdots \\
& \Sigma=S+h L+\cdots \\
& \Lambda=L+\cdots,
\end{aligned}
$$

instead of $\mathrm{Eq}$. (4). Using these relations, one finds 


$$
\frac{2 M}{\pi^{2}} E^{\prime}=\frac{3}{5}\left(6 \pi^{2}\right)^{\frac{2}{3}}\left(\frac{N}{V}\right)^{\frac{5}{3}} V+\frac{1}{5} \frac{3}{16} \pi\left(6 \pi^{2}\right)^{\frac{2}{3}}\left(\frac{N}{V}\right)^{\frac{4}{3}} S
$$

As expected the term proportional to $\mathrm{s}^{2} / \mathrm{V}$ arops out, demonstrating the discussion in Part II (Eq. (5)) that such a term is spurious, and arises from an inappropriate choice of a reference system. The surface tension coefficient is given by the coefficient of $S$ and this confirms the value obtained by Swiatecki by a different method ${ }^{10}$ ). If we had taken the coefficient of $\Sigma$ in Eq. (9) to be the surface tension coefficient we would have obtained a value which is five times too large. Thus four-fifth of this term is spurious, coming from the bulk term proportional to $\Omega$. These conclusions are independent of the values of $g$ and $h$ and do not require their knowledge. However, in order to deduce the true curvature correction coefficient for a Hill-Wheeler box (i.e., the coefficient of $I$ in $E q$. (11) rather than the coefficient of $\Lambda$ in $\mathrm{Eq} \cdot(9)$ ) a knowledge of $g$ and $h$ would be required. These numbers, characteristic of the properties of fermions in the neighbourhood of a right-angled edge, are unknown at the present time. 3.D. The Meaning of the Approximate Energy Expression

The approximate energy expression $E^{\prime}$ in terms of macroscopic quantities has been obtained by making use of two simplifications in our calculation of $I(p, 23)$. We have studied the corrections that 
should be applied to account for these simplifications. It will be shown below that both these corrections enter the energy Eq. (9) through the $\mathrm{N}^{1 / 3}$ and higher order terms, and these two corrections miraculously turn out to cancel each other to this order. Thus it will turn out that $\mathrm{E}^{\prime}$ is correct up to and including the $\mathrm{N}^{1 / 3}$ term.

The first simplification was made when we smeared each lattice point into an orthorhombic box. Thus instead of taking the energy as proportional to $\mathrm{k}_{i}^{2}$ at the lattice point, we integrated $\mathrm{k}^{2}$ over the box $\left(k_{i x} \pm w_{1}, k_{i y} \pm w_{2}, k_{i z} \pm w_{3}\right)$. This overestimates the true energy. The energy we calculated is

$$
\frac{\hbar^{2}}{2 M} \frac{1}{v} \int_{\text {box }}\left(k_{i}+\underset{\sim}{r}\right)^{2} d^{3} r=\frac{\hbar^{2}}{2 M} k_{i}^{2}+\frac{\hbar^{2}}{2 M} \frac{1}{v} \int r^{2} d^{3} r,
$$

where $\underset{\sim}{r}$ is measured from the center of the box so that $\int \underset{\sim}{r} d^{3} r=0$. (The term $\int_{\sim}^{k} \cdot \underset{\sim}{r} d^{3} r$ vanishes by symmetry.) The volume of the box is $v=\pi^{3} / a b c$. The first term gives the correct energy. Thus the correction to our calculated value is

$$
-\frac{\hbar^{2}}{2 M} \frac{1}{v} \int r^{2} d^{3} r
$$

Let us define a function $\omega(r)$ such that the portion of the box that is in the shell between $r$ and $r+d r$ is given by $\omega(r) d r$. Then the correction may be written as

$$
-\frac{\hbar^{2}}{2 M} \frac{1}{v} \int_{0}^{\infty} r^{2} \omega(x) d r
$$


The function $\omega(r)$ is zero when $r$ is greater than the distance from the center of the box to its farthest corner. The total correction $\xi_{I}$ is just the above quantity multiplied by the number of lattice points which, to the lowest order, is given by

$$
\therefore \xi_{1}=-\frac{\pi}{6} q^{3} \frac{1}{v} q^{3} \frac{1}{v^{2}} \frac{\hbar^{2}}{2 M} \int r^{2} \omega(r) d r .
$$

The second simplification was made when we smoothed out the bumps and dips by assuming a smooth effective Fermi surface of radius q (Fig. 2) this amounts to removing the bumps and filling up the dips. Since the bumps are associated with a higher energy than the dips, we have underestimated the true energy. To calculate the correction to be denoted by $\xi_{2}$, we proceed as follows. First we note that the bumps are portions of the orthorhombic boxes that stick out of the curved Fermi surface. For boxes that are at a fixed distance from the Fermi surface, we consider all the possible shapes of the bumps over the Fermi surface. Taking an average of such shapes, it turns out (see below) that we can represent an average typical bump by a series of portions of spherical shells centered at the center of the orthorhombic box with radius from zero up to the value equal to the distance from the center to the farthest corner of the box. Then we calculate the change in average energy when we remove the portion of a shell above the Fermi surface and fill up a similar portion below. Lastly we 
average this change of energy with respect to the position of the box relative to the Fermi surface. The correction $\xi_{2}$ is shown as an integral over the series of spherical shells.

Since $w_{1}\left(w_{2}, w_{3}\right)$ is múch smaller than $q$, we may consider a bump to be the portion of an orthorhombic box left when one cuts it by a plane Fermi surface at a distance $d$, say, from the center of the box. Over the Fermi surface the boxes are found to be cut in all orientations (Fig. 2). Let us assume that all orientations are equally probable, then one may describe the box averaged over orientations by specifying the amount of mattex in the shell between $r$ and $r+\Delta r$ where $r$ is measured from the center of the box. This guantity is given by

$$
\frac{I}{\mathrm{~V}} \omega(r) \Delta r,
$$

where $\omega(r)$ has been defined before in connection with the Eg. (13) for $\xi_{I}$. The factor $\frac{1}{v}$ has been included for normalisation since

$$
\int w(r) d r=v=\frac{\pi^{3}}{a b c} .
$$

For a shell between $r$ and $r+\Delta r$, consider the portion outside the Fermi surface when the center of the box is at a momentum value $k$. (See Fig. 3) This is related to $d$, the distance of the center to the Fermi surface:

$$
\mathrm{d}=\mathrm{q}-\mathrm{k} \text {. }
$$


The volume of the portion of the shell outside the Fermi. surtace is

$$
\delta=2 \pi r(r-d) \Delta r .
$$

Its energy is given by

$$
\mathrm{E}_{\mathrm{B}}=\frac{1}{\delta} \int_{0}^{\Theta} \Delta r r d \theta 2 \pi r \sin \theta(\underset{\sim}{k}+\underset{\sim}{r})^{2} \frac{r^{2}}{2 M}
$$

where $\theta_{0}$ is given by

$$
\mathrm{d}=r \cos \theta_{0}
$$

After some algebra one gets

$$
\frac{2 M}{\hbar^{2}} E_{B}=k^{2}+r^{2}+k(r+d)
$$

Now consider a dip on the Fermi surface to be a similar portion of a shell below the surface (see Fig. 3), we would be looking at a box with its centre at a momentum of value $k+2 d$. Its energy is given by

$$
\mathrm{E}_{\mathrm{D}}=\frac{1}{\delta} \int_{\pi-\theta_{0}}^{\pi} \Delta r \cdot r \mathrm{~d} \theta \cdot 2 \pi r \sin \theta(\underbrace{k+2 d}+\underset{\sim}{r})^{2 \frac{\not^{2}}{2 M}} .
$$

A similar calculation gives

$$
\frac{2 M}{\hbar^{2}} E_{D}=k^{2}+r^{2}+4 d(k+d)-(k+2 d)(r+d) .
$$

Thus the change in energy of the shell when we replace the bump by the dip is, atter simplification, 


$$
\begin{aligned}
E_{B}-E_{D} & =2(k+d)(r-d) \frac{\hbar^{2}}{2 M} \\
& =2 q(r-d) \frac{\hbar^{2}}{2 M},
\end{aligned}
$$

where we have made use of the relation, $q=k+d$.

Now we average this change in energy over all possible values of $k$ between $q-r$ and $q$. This may be done by writing the total energy change, $\Delta \mathbb{E}$, for such a shell over all the boxes on the Fermi surface (which is in the form of the curved surface of an octant) as follows:

$$
\Delta E=\int_{q-r}^{r}\left(E_{B}-E_{D}\right) \frac{\pi}{6} k^{2} d k \frac{1}{v} \text {. }
$$

Carrying through the integral, we get to the lowest order,

$$
\Delta E=\frac{\pi}{6} q^{3} \frac{1}{v} r^{2} \frac{\hbar^{2}}{2 M}
$$

This corresponds to one of the shells of radius $r$. The total correction is then

$$
\begin{aligned}
\xi_{2} & =\frac{1}{v} \int \Delta \mathrm{E} \omega(r) d r \\
& =\frac{\pi}{6} q^{3} \frac{1}{v^{2}} \frac{\hbar^{2}}{2 M} \int r^{2} \omega(r) d r .
\end{aligned}
$$

Hence we arrive at the result that to the lowest order the two corrections $\xi_{1}$ and $\xi_{2}$ add up to zero:

$$
\varepsilon_{1}+\xi_{2}=0 .
$$


It is most remarkable that the lowest order effects from two apparently unrelated sources (the replacing of lattice points by boxes in the volume of the octant, and the smoothing of bumps and dips on its surface) should cancel each other.

Let us now look at the order of the energy correction $\xi_{I}$ and $\xi_{2}$. We find that we can actually calculate $\xi_{1}$ explicitly. Equation (12) gives the correction due to the replacing of a lattice point by a box:

$$
\begin{aligned}
& -\frac{\hbar^{2}}{2 \mathrm{M}} \frac{\mathrm{l}}{\mathrm{v}} \int_{\text {box }} r^{2} \mathrm{~d}^{3} r \\
& =-\frac{\hbar^{2}}{2 \mathrm{M}} \frac{1}{\mathrm{v}} \int_{-\mathrm{w}_{3}}^{\mathrm{w}_{3}} \int_{-\mathrm{w}_{2}}^{\mathrm{w}_{2}} \int_{-\mathrm{w}_{1}}^{\mathrm{w}_{1}}\left(\mathrm{x}^{2}+\mathrm{y}^{2}+\mathrm{z}^{2}\right) \mathrm{dxdydz},
\end{aligned}
$$

where $w_{1}$, $w_{2}$, and $w_{3}$ have been defined in the last section to be half of the lengths of sides. The integration can be carried out trivially. The total correction $\xi_{1}$ in just the number of lattice points $\mathbb{N}$ multiplied by the correction due to one such case. Remembering that $v=8 w_{1} w_{2} w_{3}=\pi^{3} / a b c$, we get,

$$
\xi_{1}=-\frac{\hbar^{2}}{2 M} N \cdot \frac{1}{12}\left(w_{1}^{2}+w_{2}^{2}+w_{3}^{2}\right)
$$

Expressing this in terms of the volume, $\Omega$, surface area $\Sigma$ and integrated curvature $\Lambda$ of the Hill-Wheeler box we finally obtain after some algebra, 


$$
\xi_{1}=-\frac{\hbar^{2}}{2 M} \frac{N}{\Omega} \frac{\pi^{2}}{48}\left(\frac{1}{4} \frac{\Sigma_{1}^{2}}{\Omega}-\frac{1}{\pi} \Lambda\right) .
$$

This shows explicitly that the lowest order correction enters into the $\mathbb{N}^{1 / 3}$ term in the energy Eq. (9).

The above completes the proof that $\mathrm{E}^{\prime}$ should be able to reproduce the true energy $E$ up to the $N^{1 / 3}$ term. We show the numerical comparison of $E$ and $E^{\prime}$ in the next section.

3.E. Results and Comparisons of the Microscopic and Macroscopic

\section{Approaches}

We exhibit results from the microscopic and macroscopic approaches in this section. From the former, we obtain the exact total energy $\mathrm{E}$ (Eq. (6)). By the latter, the approximate total energy $E$ ' is calculated by successively including terms of order $N, N^{2 / 3}$, and $N^{1 / 3}(E q \cdot(7))$. In both these calculations we have assumed that $N$ is proportional to $\Omega$.

In Figs. 4a-d; we show the energies as a function of the particle number $\mathbb{N}$ for a cubic box, an oblate box, a prolate box, and a box with three unequal sides. We display the energies also as a function of the deformation parameter $\alpha$ (putting $\gamma=0$ ) in Figs. $5 a, b$, which is on a somewhat larger scalet. In the latter case two

+ The exact results $E$ as a function of deformation are the lowest possible energies at each deformation. They correspond to an adiabatic process of deforming the Hill-Wheeler box. 
systems are studied, one with $\mathbb{N}=60$ when a shell occurs at zero deformation, and one with $N=68$ where no shell occurs at zero deformation.

When we calculate $E^{\prime}$ only up to the volume term, i.e., the $N$-term, we find that the results give (at $N=60$ ) about $70 \%$ of $E$. We can nake a correspondence between our calculated energies with the realistic nuclear energies, by requiring the density of particles in the box to be given by nuclear matter density (corresponding to a radius constant $r_{\mathrm{O}}=1.2 \mathrm{fm}$ ). We remember also that we are filling every energy level with one particle whereas in the nuclear case there are two protons and two neutrons in each level. It then turns out that the calculated total energy for $N=60$ in the case of zero deformation, corresponds to $6914 \mathrm{MeV}$ in a nucleus of $A=240$; the energy corresponding to the $\mathrm{N}$-term is $4830 \mathrm{MeV}$. The figures also shows that the difference increases with increasing $\mathrm{N}$. As a function of deformation $\alpha, \mathrm{E}^{\prime}$ is a constant (not shown in the figures), whereas $E$ increases with deformation.

The inclusion of the $\mathrm{N}^{2 / 3}$ term in the calculation of $\mathrm{E}^{\prime}$ improves the picture substantially. Values of $E^{\prime}$ are still smaller than $E$ but the difference is less. At $N=60, \mathrm{E}^{\prime}$ is $6675 \mathrm{MeV}$ which accounts for $97 \%$ of the true value. The difference is a less rapidly varying function of $N$. Also as a function of deformation, 
$\mathrm{E}$ ' now represents the trend of $\mathrm{E}$ fairly well ${ }^{\dagger}$, though there is a difference in absolute values. In Figs. $6 a-d$ and $7 \mathrm{a}, \mathrm{b}$ we plot the differences between $E$ and $E^{\prime}$ both as a function of $\mathbb{N}$ and as a function of $\alpha$ on a much expanded scale. The differences are shown to be increasing slightly with $\mathbb{N}$ or $\alpha$.

The further inclusion of $N^{1 / 3}$ term in the calculation of $E^{\prime}$ seems to be capable of reproducing the exact energy $E$ very well. In Figs. 4 and 5 there appears to be no difference at all on the scale used. In the Figs. 6 and 7 where the differences of $E$ and $E$ ' are plotted on a much expanded scale we find that $E^{+}$is still slightly below $E$. The mean difference over $\mathbb{N}$ values is only about $14 \mathrm{MeV}$ putting $E^{\prime}$ within $99.8 \%$ of the exact value. The difference is expected to be in the $\mathbb{N}^{\circ}$ term, and we find indeed that it does appear to be constant as a function of $\mathbb{N}$. It also appears to be fairly constant as a function of $\alpha$, apart from local fluctuations. The strong convergence of the various terms in the macroscopic result $E^{\prime}$ to the exact result $\mathrm{E}$ is illustrated by listing below the contributions from these terms for the case of $N=60$ :

+ Hill and wheeler ${ }^{6)}$ in their work show a graph which appears to indicate that the trend of $E^{\prime}$ to order $\mathbb{N}^{2 / 3}$ is quite different from that of E. However as was pointed out by Myers and Swiatecki ${ }^{4}$, there seems to be a mistake in their plot though their equations are correct. 


\begin{tabular}{lr}
$\mathrm{N}$-term & $4830 \mathrm{MeV}$ \\
$\mathrm{N}^{2 / 3}$-term & $1845 \mathrm{MeV}$ \\
$\mathrm{N}^{1 / 3}$-term & $225 \mathrm{MeV}$ \\
$\mathrm{N}^{0}$-term + Rest & $14 \mathrm{MeV}$ \\
\hline Exact result E & $6914 \mathrm{MeV}$
\end{tabular}

It thus appears that by including enough terms in $E^{\prime}$, the exact result $E$ may be reproduced very closely by the macroscopic calculation. Let us study further the difference between the true result $\mathrm{E}$ and the result. $E^{\prime}$ where terms up to the $\mathbb{N}^{1 / 3}$ term have been included. In Figs. 6 and 7 we see a wiggly structure in the differences both as a function of $\mathbb{N}$ and as a function of $\alpha$. A dip in energy occurs where there is a shell. Thus the difference between $E$ and $E$. furnishes a convenient way of studying the shell effects. For zero deformation (Fig. 4a) we find shells at $\mathbb{N}=1,4,17,35,38,60, \cdots$ These correspond to gaps in the single particle level diagram (Fig. I). We note that the occurrence of shells is associated with a given deformation. For instance, the $\mathrm{N}=60$ shell for zero deformation is completely removed when the shape becomes prolate $(\alpha=0.25, \quad r=0)$ as shown in Fig. 4c. In this prolate case shells appear at $N=7,14$, $27,54, \cdots$, bearing little resemblance to the positions of shells at zero deformation. 
3.F. Summary and Conclusions

In this part of the thesis we have considered the model of noninteracting spinless Fermions in a Hill-Wheeler box. The exact values of the total energy as a function of the particle number $N$ and the shape of the box were calculated. This is a prototype of the microscopic Nilsson model calculation ${ }^{2)}$. In the macroscopic approach the energy is found as a liquid drop type of expression, i.e., as an expansion in $\mathrm{N}^{-1 / 3}$ with terms dependent on the volume, surface area, and integrated curvature of the system. It is found that as one successively includes terms of orders $N, N^{2 / 3}$, and $N^{1 / 3}$, the results converge very quickly to the smooth average of the exact results.

Hence we see that the liquid drop type of expression for the energy is applicable even in the present case which assumes no interactions between particles and is in fact a pure shell model. The applicability is based only on the fact that the system we are considering is leptodermous.

In application to nuclear problems the liquid drop type of expression is usually truncated at the leading volume, surface, (and curvature) terms. A question may be asked how bad is such a truncation. This has been discussed by swiatecki ${ }^{13}$ ). The main point is that in practice the coefficients of these leading terms are adjustable parameters chosen so that the masses of all nuclei in the periodic table as well as the known fission barriers are approximately reproduced. This means that any smoothly varying higher order terms are partly absorbed in the leading terms. Although this compensation cannot be perfect, 
only a fraction of these smooth higher order terms will not be accounted for. On the other hand, rapidly oscillating terms, such as the single particle shell corrections neither remain constant throughout a nuclear deformation, nor can they be absorbed in the smooth leading term.

All these discussions lead one to a hypothesis that for a nucleus (which is a leptodermous system: See Part II), the liquid drop type of energy expression with the first few terms gives correctly the smooth average trends both as a function of the nucleon number and as a function of the deformation. The single particle shell effects may be considered as local wiggles superimposed on these smooth trends. This is the basic philosophy behind the method of the synthesis of a microscopic model and a macroscopic model that will be presented in Part $V$ of this thesis. 
4. On the Symmetric Saddle Point Configurations of a Charged Conducting Drop

4.A. Introduction

In this part of the thesis, we turn, to a pure liquid drop model study of fission of a charged conducting drop whose charges reside on its surface. Such a model is in contrast to the usual liquid drop model. of nuclear fission which assumes a liquid drop with charges uniformly distributed throughout its volume. Nevertheless it is interesting to carry out a theoretical and an experimental investigation on a charged conducting drop as a parallel to the theoretical and experimental study of nuclear fission. Beginnings in this direction have been made ${ }^{14}$ ). Furthermore the charged conducting drop is also interesting for its own sake and for its role in the cloud physics and other fields ${ }^{15}$ ).

In 1882 Lord Rayleigh published a paper on the stability of a charged conducting sphere ${ }^{16)}$. If one had combined the results of this work with the semi-empirical nuclear mass formula due to Weizsäcker ${ }^{17}$ ) in 1935, one would have been led to expect nuclear fission. It even turns out that the criterion for the stability of a charged conducting drop is identical with the criterion for the stability of the nucleus against fission. Ryce and collaborators ${ }^{14}$ in 1964,1965 , and 1966 considered some simple aspects in the splitting of a conducting drop. They looked at only the initial and final stages of the fission and speculated on features that could possibly be applied to nuclear fission. Very recently at the International Symposium on Ellectrohydrodynamics (1969) more studies ${ }^{15}$ ) on the charged conducting drop were reported. 


$$
-43-
$$

It is the purpose of this present work to study the first stage of the theory of fission of a conducting drop, i.e., its statics. In particular we have determined approximately, the most important symmetric equilibrium configurations of the drop. The similarities and differences of the conducting drop and a volume charged drop are also discussed. 4.B. Basic Concepts in Fission Theory

In this section we shall review some basic concepts and results in the theory of nuclear fission ${ }^{18)}$. For an incompressible volume charged drop, two forces are acting: a coulomb force which tends to break up the drop and a surface tension which tends to keep it together. A quantity of importance is then the ratio of the Coulomb energy and the surface energy. One may define what is called the fissility parameter, $\mathrm{x}$, as

$$
x=\frac{E_{C}^{(0)}}{2 E_{S}(0)} \propto \frac{Q_{1}^{2} / R}{R^{2}} \propto \frac{Q_{1}^{2}}{V},
$$

where $\mathrm{E}_{\mathrm{C}}(\mathrm{O})$ and $\mathrm{E}_{\mathrm{S}}(\mathrm{O})$ are Coulomb and surface energies of a sphere with charge $Q$, radius $R$, and volume $V$. For $x<1$, the spherical arop is stable with respect to deformations and for $x>1$, the coulomb force is greater than the surface tension and the drop is unstable. Let us write down the energy excess of a deformed drop over the original spherical drop as

$$
\begin{gathered}
E_{S}-E_{S}^{(0)}+E_{C}-E_{C}^{(0)}=E_{S}^{(0)}\left(B_{S}-1\right)+E_{C}{ }^{(0)}\left(B_{C}-1\right) \\
=E_{S}{ }^{(0)}\left\{\left(B_{S}-1\right)+2 x\left(B_{C}-1\right)\right\},
\end{gathered}
$$


where $E_{S}$ and $E_{C}$ are the surface and coulomb energies of the drop and the superscript (o) implies that the quantity is evaluated for the case of a sphere. Also $B_{S}=E_{S} / E_{S}(0)$ and $B_{C}=E_{C} / E_{C}(0)$. If we define $\xi$ as the energy excess in units of $\mathbb{E}_{\mathrm{S}}(0)$ then $\xi$ is just the above expression divided by $\mathrm{E}_{\mathrm{S}}(\mathrm{O})$

$$
\xi=B_{S}-1+2 x\left(B_{c}-1\right)
$$

In Fig. 8, we sketch the behavior of $\xi$ as a function of deformation for a particular value of $x<1$. The configuration at zero deformation, i.e., a sphere, is a potential energy minimum. The energy is increased as one deforms the drop until a point is reached where the disruptive Coulomb force is dominant and the drop undergoes fission. The configuration corresponding to the point where the drop will start dividing of its own accord is called the saddle point shape. It is unstable with respect to the deformation leading to fission. Obviously the curve will be different for different values of charge on the drop, i.e., different values of $x$ (see Fig. 9). Thus for $x>1$, the sphere is at a potential maximum.

Let $\xi_{\mathrm{R}}$ denote the difference in energy between the initial sphere and the final fragments at infinity in units of $E_{S}(0)$. For division into two equal spheres which is illustrated in the figure, $\xi_{\mathrm{R}}=0$ at $\mathrm{x}=0.35$. For $\mathrm{x}>0.35, \xi_{\mathrm{R}}<0$, and for $\mathrm{x}<0.35$, $\xi_{R}>0$. In the general case of division into $n$ equal spheres, a general. formula ${ }^{19)}$ may be written for $\xi_{R}$. The charge on each sphere 
is $Q / n$ and its radius is $\left(\mathrm{R}^{3} / \mathrm{n}\right)^{1 / 3}=\mathrm{R}^{-1 / 3}$, so that the Coulomb energy of the $\mathrm{n}$ spheres is $\mathrm{n}$ multiplied by the coulomb energy of each sphere:

$$
\begin{aligned}
E_{C} & =n \frac{3}{5} \frac{(Q / n)^{2}}{R n^{-1 / 3}} \\
& =\frac{3}{5} \frac{Q}{R} n^{-2 / 3} \\
\therefore B_{C} & =n^{-2 / 3} .
\end{aligned}
$$

Total surface energy of the $n$ spheres is

$$
\begin{gathered}
E_{s}=r n \cdot 4 \pi\left(\mathrm{R} \mathrm{n}^{-1 / 3}\right)^{2}=4 \pi \mathrm{R}^{2} \mathrm{~m}^{1 / 3} \\
\therefore \mathrm{B}_{\mathrm{S}}=\mathrm{n}^{1 / 3} .
\end{gathered}
$$

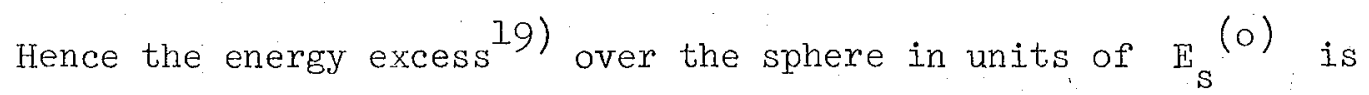

$$
\xi_{R}=\left(n^{1 / 3}-1\right)+2 x\left(n^{-2 / 3}-1\right)
$$

This is shown in Fig. 10, where the energy release $\xi_{\mathrm{R}}$ is plotted against $x$ for division into two, three, four, up to eight equal spheres. For each value of $n$, the plot is a straight line. The straight line for $n=2$ goes through zero at $x=0.35$. For $x<0.35$, the sphere has the lowest energy. For $0.35<x<0.61$, the division into two spheres gives the lowest energy. For $0.61<\mathrm{x}<0.87$, the 
division into three equal spheres gives the lowest energy. Finally, for $0.87<\mathrm{x}<1.12$, the division into four equal spheres gives the lowest energy.

In fig. 11, we present the shapes of a volume charged drop at the saddle point as found by $\mathrm{Nix}^{20}$ ), so that we can compare it with the results we are going to obtain for a surface charged drop. The abscissa gives the fissility parameter $x$ from 0 to 1 . The ordinate gives $\mathrm{R}_{\mathrm{MIN}} / \mathrm{R}$ and $\mathrm{R}_{\mathrm{MAX}} / \mathrm{R}$ as a measure of the shape, where for an asymmetric shape radius $R_{M I N}$ is the minimum radius of the neck of the drop and the two maximum radii $R_{\text {MAX }}$ are the distances from the center of the neck (at its minimum radius) to the two ends of the drop. For a symmetric shape the two maximum radii are equal.

Along $\mathrm{R}_{\mathrm{MAX}} / \mathrm{R}=1$ is the sphere which is at a potential energy minimum for all $x<1$. The rest of the curves represent a family of reflection symmetric saddle point shapes and a family of reflection asymmetric saddle point shapes. The two families cross each other at $x=0.396$. Their shapes are schematically indicated in the figure. A point to notice is that along the symmetric family there is a fairly rapid change in the trend of $R_{\text {MAX }} / R_{O}$ at $x$ values around 0.7 . It is found below (Sec. F) that for a conducting drop a similar change occurs at a larger value of $x$. 4.C. Comparison of a Conducting Drop and a Volume Charged Drop

In the last section we have reviewed some basic properties of a volume charged drop. In this section we shall point out some similarities and differences in the properties of a conducting drop and of a 
volume charged drop. For a conducting drop the fissility parameter $x$ can be similarly defined as the ratio of the Coulomb energy to twice the surface energy evaluated for a conducting sphere. The equation (14) for the energy excess $\xi$ will be the same as for the volume charged drop case except that the Coulomb energies will now be evaluated on the assumption that the drop is conducting.

Let us first consider the similarities.

(1) For $x=0$, there is no charge on the drop so that the equilibrium shapes are the same whether the drop is conducting or not. For $\mathrm{x}=1$, it turns out nontrivially that as in the case of a volume charged $\mathrm{drop}^{21}$ ), the Coulomb force is just balanced by the surface tension for a spherical conducting drop.

(2) A second similarity is apparent if we look at the energy difference $\xi_{R}$ from the initial to the final state when the drop is divided into equal spheres. We have described this in detail for a volume charged drop in reference to Fig. 10. When we make a similar study for a conducting drop, we get completely identical straight lines and conclusions. The reasion is that only spherical shapes are involved in both the initial and final states, and the Coulomb energy of a volume charged sphere (which is $\frac{3}{5} Q^{2} / R$ ) and that of a conducting sphere (which is $\frac{1}{2} Q^{2} / R$ ) differ by only a numerical factor, $6 / 5$, that is the same for both states. Hence $B_{S}$ and $B_{C}$ are the same for both cases and the same energy Eq. (15) holds good.

(3) It also turns out that the Coulomb energy of a volume charged ellipsoid and that of a conducting ellipsoid differ also by the 
same numerical factor. Thus the Coulomb energy of a conducting ellipsoid is given by ${ }^{22)}$

$$
E_{c}=\frac{1}{4} Q^{2} \int_{0}^{\infty}\left[\left(a^{2}+\lambda\right)\left(b^{2}+\lambda\right)\left(c^{2}+\lambda\right)\right]^{-\frac{1}{2}} d \lambda
$$

so that

$$
B_{c}=\frac{1}{2} R \int_{0}^{\infty}\left[\left(a^{2}+\lambda\right)\left(b^{2}+\lambda\right)\left(c^{2}+\lambda\right)\right]^{-\frac{1}{2}} d \lambda
$$

where $a, b$, and $c$ are the lengths of the axes of an ellipsoid. The $B_{C}$ for a volume charged case turns out to be the same ${ }^{23)}$. In the case of a prolate spheroid $b=c$ we get on integration

$$
B_{c}=R \frac{1}{2 a e} \ln \left(\frac{1+e}{1-e}\right)
$$

where $e^{2}=1-a^{2} / c^{2}$. Since $R^{3}=a c^{2}$, we get

$$
B_{c}=\frac{1}{2}\left(1-e^{2}\right)^{1 / 3} \frac{1}{e} \ln \left(\frac{1+e}{1-e}\right) .
$$

For an oblate spheroid, we can just set $e$ to ie and $e^{2}$ to $-e^{2}$, and find

$$
B_{c}=\left(I+e^{2}\right)^{I / 3} \frac{1}{e} \tan ^{-1} e .
$$

These expressions for $B_{c}$ hold good for both a volume charged drop and a conducting drop. Hence if we make the drop to take on only ellipsoidal shapes, then any conclusions about the statics of the volume charged drop will be true for the conducting drop. 
Now let us look at some differences between the two cases. The first difference between the volume charged drop and a conducting drop can be found if we consider the division of the drop into two unequal spheres at an infinite distance apart, one with volume $\beta V$ and the other with volume $(1-\beta)$ V. In Fig. 12 is plotted the energy change $\xi_{R}$ between the initial and final states ${ }^{24}$ as a function of $\beta$ for various values of the fissility parameter $x$. For $\beta=0$ and $\beta=1$ we get a sphere with volume $V$ which is just the initial state. For $\beta=0.5$, we get two equal spheres. The energy change is zero at $\mathrm{x}=0.35$ for $\beta=0.5$, as was pointed out above in connection with Fig. 10. For a conduction drop Fig. 13 is found ${ }^{24)}$. We note that here again the energy is zero at $x=0.35$ for $\beta=0.5$ consistent with our previous statement that Fig. 10 also applies to a conducting drop. Except for the points at $\beta=0,0.5$, and 1.0 the curves in the two figures are very different. A potential minimum for a volume charged drop occurs at $\beta=0.5$ for $x>0.2$, but a potential maximum for a conducting arop occurs at $\beta=0.5$ for all $x$ values less than one. In the latter case minima occur at points where the fragments are unequal.

The major reason for the above difference is that the charge to mass ratio for the volume charged drops is a constant, but for the conducting drops it is not required to be a constant. This is also the underlying cause for the second difference that appears when we try to find the configuration with the absolute lowest energy for a drop with a given fissility parameter $x$. For a volume charged drop, this configuration is $n$ equal droplets at infinity ${ }^{19}$ ) and the number $n$ 
depends on the $x$ value of the drop [Eq. (15)]. One would at first expect that the same conclusion might hold for a conducting drop. But, as we shall show, for a conducting drop, the configuration at the lowest energy is one with all the charges $Q$ on the drop taken off and distributed among many infinitesimal droplets at infinity. It turns out that the total energy of the droplets may be made to vanish and we are just left with the surface energy of the original drop. The possibility of such a configuration is show as follows. Let $\frac{I}{n}$ of the original drop of radius $R$ be taken off carrying all the charge $Q$. This is then divided into $m$ equal spheres, each with a charge $Q / m$. Thus for each sphere the sum of the Coulomb and surface energy is

$$
4 \pi R^{2} r\left(\frac{1}{n m}\right)^{2 / 3}+\frac{1}{2} \frac{Q_{1}^{2}}{R}(n m)^{1 / 3}\left(\frac{1}{m}\right)^{2} \text {. }
$$

Hence the total energy of the small spheres is $m$ times this quantity:

$$
\begin{aligned}
& 4 \pi R^{2} r m(n m)^{-2 / 3}+\frac{1}{2} \frac{Q^{2}}{R}(n m)^{1 / 3} m^{-2} \cdot m \\
& =4 \pi R^{2} r m^{1 / 3} n^{-2 / 3}+\frac{1}{2} \frac{Q^{2}}{R} n^{1 / 3} m^{-2 / 3} .
\end{aligned}
$$

Now let us choose $\mathrm{m}^{-\mathrm{s}} \mathrm{n}^{-\mathrm{S}}$. The energy of the droplets is now equal to

$$
4 \pi R^{2} r n^{-\frac{2}{3}-\frac{s}{3}}+\frac{1}{2} \frac{Q^{2}}{R} n^{\frac{1}{3}}+\frac{2 s}{3}
$$

which is zero when $\mathrm{n}$ goes to infinity provided

$$
-2<s<-\frac{1}{2}
$$


and we obtain the proposed configuration. In other words, we have made the Coulomb energy of the given drop zero by dispersing the charges onto an infinite number of infinitesimal droplets without increasing the surface energy by a finite amount. 4.D. Parameterization of a Conducting Drop

In the remainder of this part of the thesis we shall try to determine the equilibrium shapes of a charged conducting drop. The calculation of the coulomb energy of a conducting drop with an arbitrary shape is in general a difficult problem. We have side-stepped this difficulty by requiring the drop to assume a prescribed family of shapes, and have in fact made the calculation of its Coulomb energy a trivial matter. It is well-known from the theory of electrostatics ${ }^{25}$ ) that the electric potential of any system of charges is the same at every point outside any equipotential which surrounds all the charges, as that of the same total charge spread over a conductor that has the shape of this equipotential. Hence we require the drop to assume the shape of an equipotential of potential $\alpha$ due to a system of point charges with total charge $Q$. Then if we put the charge $Q$, on this conducting drop, its potential is equal to $\alpha$, and its Coulomb energy is just $\frac{1}{2} \alpha Q$.

Consider an example of two equal point charges. The shapes of equipotentials that enclose the point charges are shown in Fig. 14, where the volumes of the shapes have been normalised to the same value. We shall refer to these shapes as the symmetric $\mathbb{N}=2$ family since 
they are generated with two point charges and are reflection symmetric. Each of these equipotentials is associated with a potential $\alpha$. Then the coulomb energy of a drop with this shape is $\frac{1}{2} \alpha Q$, where $Q$, is the charge on the drop. If $R$ is the radius of a sphere that has the same volume as the drop and possesses the same amount of charge, its Coulomb energy is $\frac{1}{2} Q_{1}^{2} / R$. Hence we get

$$
B_{C}=\alpha R / Q
$$

The surface energy relative to that of the sphere $B_{S}$ can simply be found by calculating its area numerically. Hence for a given fissility $\mathrm{x}$ the energy of the drop is calculated [Eq. (14)]. Equilibrium shapes are then the shapes whose energy is stationary.

The symmetric $N=2$ family has only a very restricted series of shapes. However it is easy to increase the possible shapes by looking instead at the shapes that correspond to the equipotentials of a larger number of point charges. We have put the charges on a straight line so that all our shapes remain axially symmetric. The reflection symmetric $N=3$ family is generated with two equal charges situated at equal distances on opposite sides of a third point charge. The shapes are shown in Fig. 15. They include the symmetric $N=2$ family. Similarly we can go on to $N=4,5, \cdots$ family of shapes.

Let us consider in general the $\mathrm{N}$-family of axially symmetric shapes. To specify the situation we need to give the magnitudes of the iv point charges and their positions as well as the value of the potentint wn the equipotential we are looking at. These are $2 \mathrm{~N}+1$ numbers. 
However not all these numbers are required to specify a shape. Three numbers may be arbitrary: (1) The center of mass of all the point charges may be at any point in space; (2) The total charge may be fixed beforehand; (3) We can also preset a scale by which the distances between the point charges are measured. The first point just puts the drop at any place in space, while the last two points just introduce a scaling factor into the volume of the shape, which will be taken care of when we calculate $B_{S}$ and $B_{C}$ eventually. Hence we set the sum of all the point charges to be unity:

$$
a_{1}+a_{2}+\cdots+q_{N}=1
$$

We also place the charges on a horizontal axis with the end charges at positions -0.5 and +0.5 with respect to the origin, and specify the relative positions of the other charges in units of the distance between the end charges.

$$
\begin{aligned}
-0.5 \leq b_{i} & \leq 0.5 \\
b_{1} & =-0.5 \\
b_{N} & =0.5 .
\end{aligned}
$$

Thus we are left with $2 \mathrm{~N}-2$ parameters. (For reflection symmetric shapes, the distribution of point charges and their magnitudes are reflection symmetric with respect to the origin and we have only $\mathbb{N}-1$ parameters.) 
Let $u_{i}$ be the distance from the point charge $i$ to any point on the equipotential surface. Then a point on this surface may be specified by the symmetric and antisymmetric variables $v$ and $w$ given by

$$
\begin{aligned}
& v=u_{1}+u_{N} \\
& w=u_{1}-u_{N} .
\end{aligned}
$$

The surface of the drop cuts its axis at $w= \pm 1$ and cuts the plane through the origin perpendicular to its axis at $w=0$. The distance, $y$, of this point from the axis and its position along the axis $z$, as well as $u_{i}$ and the value of the electric potential $\alpha$ can be found to be given in terms of $v$ and $w$ as follows:

$$
\begin{aligned}
z & =\frac{1}{2} v w \\
y^{2} & =\frac{1}{4}\left(1-w^{2}\right)\left(v^{2}-1\right) \\
u_{i}^{2} & =\frac{1}{4}\left(v^{2}+w^{2}\right)-v w l_{i}-\left(\frac{1}{4}-\ell_{i}^{2}\right) \\
\alpha & =\sum_{i=1}^{N} \frac{q_{i}}{u_{i}}
\end{aligned}
$$

or

$$
\alpha=\sum_{i=1}^{N} \frac{g_{i}}{\left[\frac{1}{4}\left(v^{2}+w^{2}\right)-v w b_{i}-\left(\frac{1}{4}-b_{i}^{2}\right)\right]^{\frac{1}{2}}}
$$


Since $\alpha$ is given as one of the parameters, we can use the last equation to solve for $v$ when $w$ is given.

The total volume $V$ and surface area $S$ can be written down

$$
\begin{aligned}
V & =\int \pi y^{2} \frac{d z}{d w} d w \\
S & =\int 2 \pi y\left(d z^{2}+d y^{2}\right)^{\frac{1}{2}} \\
& =\int 2 \pi\left[y^{2}\left(\frac{d z}{d w}\right)^{2}+\frac{1}{4}\left(\frac{d}{d w} y^{2}\right)^{2}\right]^{\frac{1}{2}} d w .
\end{aligned}
$$

Then we can find $R$ from

$$
\frac{4}{3} \pi R^{3}=V
$$

and

$$
\begin{aligned}
& B_{S}=\frac{S}{4 \pi R^{2}} \\
& B_{c}=\alpha R .
\end{aligned}
$$

We shall find later that we require the curvature $\kappa$ and the electric field $\mathcal{E}$ at any point on the surface, which may be shown to be given by

$$
\begin{aligned}
\varepsilon^{2} & =\mathrm{C}_{0}^{2} \mathrm{y}^{2}+\left(\mathrm{c}_{0} z-\mathrm{C}_{1}\right)^{2} \\
\kappa & =\frac{8 \mathrm{y}^{2}+4\left[\left(\mathrm{y}^{2}\right)^{\prime}\right]^{2}-4 \mathrm{y}^{2}\left(\mathrm{y}^{2}\right)^{\prime \prime}}{\left(4 \mathrm{y}^{2}+\left[\left(\mathrm{y}^{2}\right) \cdot\right]^{2}\right)^{3 / 2}},
\end{aligned}
$$


where the prime superscripts denote derivatives with respect to $z$. The coefficients $C_{0}$ and $C_{1}$ as well as the derivatives that enter into these equations are listed below:

$$
\begin{aligned}
\frac{d v}{d w} & =\frac{-\frac{1}{2} w C_{0}+v C_{1}}{\frac{1}{2} v C_{0}-w C_{1}} \\
\frac{d z}{d w} & =\frac{1}{2}\left(v+w \frac{d v}{d w}\right) \\
\frac{d\left(y^{2}\right)}{d w^{2}} & =\frac{1}{2}\left(1-w^{2}\right) v \frac{d v}{d w}-w\left(v^{2}-1\right) \\
\frac{d\left(y^{2}\right)}{d z} & =2\left(\frac{C_{1}}{C_{0}}-z\right) \equiv \frac{d\left(y^{2}\right) / d w}{d z / d w} \\
\frac{d^{2}\left(y^{2}\right)}{d z^{2}} & =-2+\frac{6}{C_{0}}\left(D_{2}-\frac{C_{1} D_{1}}{C_{0}}\right)-\frac{6 C_{1}}{C_{0}^{2}}\left(D_{1}-\frac{C_{1} D_{0}}{C_{0}}\right)
\end{aligned}
$$

where

$$
\begin{aligned}
& \mathrm{C}_{0}=\sum q_{i} / u_{i}^{3} \\
& \mathrm{C}_{1}=\sum q_{i} b_{i} / u_{i}^{3} \\
& D_{0}=\sum q_{i} / u_{i}^{5} \\
& D_{1}=\sum q_{i} b_{i} / u_{i}^{5} \\
& D_{2}=\sum q_{i} b_{i}^{2} / u_{i}^{5} .
\end{aligned}
$$


4.E. The Determination of Equilibrium Shapes

In our numerical calculations, we have restricted the drop to assume only reflection symmetric shapes. Thus we have chosen the magnitudes and positions of the point charges such that they are reflection symmetric with respect to the origin. As discussed in the last section, a total of $N-I$ parameters will specify the shape. Let us denote them by $p=\left(p_{1} p_{2} \cdots p_{N-1}\right)$. The energy of a conducting drop with fissility $x$ in units of the surface energy of a sphere of equal volume is then

$$
\xi(p)=B_{s}-1+2 x\left(B_{c}-1\right)
$$

Let $f_{i}$ be the derivative of $\xi$ with respect to $p_{i}$. Then $f_{i}(\bar{p})=0$ if $\bar{p}$ represents the equilibrium shape. Expanding $f_{i}(\bar{p})$ about the parameters $\mathrm{p}$, we have,

$$
f_{i}(\bar{p})=f_{i}(p)+\sum_{j} \Delta p_{j}\left(\frac{\partial f_{i}}{\partial p_{j}}\right)
$$

to the first order. Since the left-hand-side is zero,

$$
f_{i}(p)=-\sum_{j} \Delta p_{j} \frac{\partial f_{i}}{\partial p_{j}}
$$

The factors $\Delta p_{j}$ are the differences of the parameters $p$ from the equilibrium values $\bar{p}$. Thus for a given fissility $x$, a first guess of the parameters $p$ close to the equilibrium values is made, and $f_{i}$ and its first derivatives are calculated numerically. Then solving the 
system of simultaneous Iinear Eqs. ( 177$), \quad(i=1,2, \cdots, N-1)$, we obtain corrections $\Delta p_{j}$ to the guessed values. Corrections to successive guesses are found until they are less than a prescribed accuracy. Then the parameters finally obtained are assumed to describe an equilibrium shape and the energy of the drop is calculated. By calculating and diagonalising the second derivatives of the energy with respect to all the parameters, we find the number of co-ordinates with respect to which the equilibrium point is a maximum and the number of co-ordinates with respect to which it is a minimum.

The shapes generated even by a large number of point charges are not general enough to represent an arbitrary shape. Thus an oblate shape cannot be found in our scheme. This raises the question whether the equilibrium shapes we have determined are indeed true equilibrium configurations when the drop is free to take on any arbitrary shape. To answer this question a criterion will be developed to test a given shape for equilibrium. (A similar criterion exists for a volume charged $\mathrm{drop}^{21}$.)

If we deform a conducting drop at equilibrium by specifying a normal displacement on of the surface element ds without affecting its total charge, the coulomb energy change is found to be 25 )

$$
\delta \mathrm{E}_{\mathrm{C}}=-\int \frac{1}{2} \sigma C^{\prime} \text { in } \mathrm{ds}
$$

The change in surface energy may also be found: 


$$
\delta \mathrm{E}_{\mathrm{S}}=r \int \kappa \delta \text { in } \mathrm{d} S
$$

where $r$ is the surface tension coefficient. The total energy change is

$$
\delta \mathrm{E}^{-} \delta \mathrm{E}_{\mathrm{C}}+\delta \mathrm{E}_{\mathrm{S}}
$$

Subtracting SndS times a Lagrange multiplier $k$ to ensure conservation of volume and equating the integrand to zero (for equilibrium shapes) gives

$$
k=r \kappa-\frac{1}{2} \sigma \ell .
$$

By Gauss' Theorem,

$$
\begin{aligned}
\sigma & =\frac{1}{4 \pi} \xi \\
\therefore \quad k & =r \kappa-\frac{\varepsilon^{2}}{8 \pi} \\
& =r \kappa_{0}\left(\frac{\kappa}{\kappa_{0}}-\frac{\xi_{0}^{2}}{8 \pi r \kappa_{0}} \frac{\varepsilon^{2}}{\delta_{0}^{2}}\right)
\end{aligned}
$$

where we have introduced $k_{0}$ as the curvature on a sphere with the same volume as the arop and $E_{0}$ to be the electric field on the sphere. Since 


$$
\begin{aligned}
& -60- \\
& x=\frac{E_{c}^{(0)}}{2 E_{s}(0)}=\frac{1}{n_{0}} \frac{E_{0}^{2}}{8 \pi} \\
& \therefore k=\gamma_{0}\left(\frac{\kappa}{\kappa_{0}}-x \frac{\zeta^{2}}{\varepsilon_{0}^{2}}\right) .
\end{aligned}
$$

The Iagrange multiplier $\mathrm{k}$ is determined by considering the effect of a uniform change of scale (while keeping $Q$, constant) on the shape satisfying Eq. (18). If $\delta \mathrm{m}$ is the nonvolume-preserving displacement of the surface associated with the change of scale and SV is the corresponding volume change, Then

$$
\delta \mathrm{E}=\int\left(\gamma_{k}-\frac{1}{2} \sigma \hat{C^{h}}\right) \delta \mathrm{m} d \mathrm{~S}=\mathrm{k} \int \delta \mathrm{mdS}=\mathrm{k} \delta \mathrm{V} .
$$

On the other hand by dimensional considerations,

$$
\begin{aligned}
\mathrm{E}_{\mathrm{S}} & \propto \mathrm{V}^{2 / 3} \\
\mathrm{E}_{\mathrm{c}} & \propto \mathrm{V}^{-1 / 3} \\
\therefore \mathrm{E}(\mathrm{V}+\delta \mathrm{V}) & =\mathrm{E}_{\mathrm{c}}\left(\frac{\mathrm{V}}{\mathrm{V}+\delta \mathrm{V}}\right)^{1 / 3}+\mathrm{E}_{\mathrm{S}}\left(\frac{\mathrm{V}+\delta \mathrm{V}}{\mathrm{V}}\right)^{2 / 3} \\
\therefore \quad \mathrm{E} & =-\frac{1}{3} \mathrm{E}_{\mathrm{c}} \frac{\delta \mathrm{V}}{\mathrm{V}}+\frac{2}{3} \mathrm{E}_{\mathrm{S}} \frac{\delta \mathrm{V}}{\mathrm{V}}
\end{aligned}
$$

Comparing with Eq. (19),

$$
\therefore \mathrm{k}=-\frac{1}{3} \mathrm{E}_{\mathrm{c}} \frac{\mathrm{I}}{\mathrm{V}}+\frac{2}{3} \mathrm{E}_{\mathrm{S}} \frac{\mathrm{I}}{\mathrm{V}} .
$$


Expressing all quantities in units of their values for a sphere we get after some algebra,

$$
k=r \kappa_{0}\left(B_{S}-x B_{C}\right) \text {. }
$$

Comparing Eqs. (18) and (20), we get

$$
\frac{\frac{\kappa}{\kappa_{0}}-x \frac{\xi^{2}}{\xi_{0}^{2}}}{B_{S}-x B_{C}}=1 .
$$

Thus for an equilibrium shape, any point on its surface should satisfy $\Delta=0$, where $\Delta$ is given by

$$
\Delta=\frac{\frac{\kappa}{\kappa_{0}}-x \frac{\xi^{2}}{\xi_{0}^{2}}}{B_{S}-x B_{c}}-1 .
$$

As a measure of the deviation from equilibrium we can define a rootmean-square value of $\Delta$ over the surface of the drop:

$$
\operatorname{RMS}=\left(\int|\Delta|^{2} \mathrm{~d} S\right)^{\frac{1}{2}} \cdot
$$

If RMS $\ll 1$, the drop is close to equilibrium. If RMS $>1$, the shape is far from equilibrium. This quantity will be used as a measure of how close the shapes we have determined are to the true equilibrium. 


\section{F. Results}

The results for symmetric equilibrium shapes of a charged conducting drop based on a family of shapes generated by two, three up to six point charges are shown in Fig. 16 as a series of curves. The figure is equivalent to Fig. Il for a volume charged drop. The series of curves with different $N$ values are just successive orders of approximation of the true equilibrium shapes. One would hope that for a high enough order of approximation, the results may be very close to the true ones, so that an even higher order will change the results very little. Typically, for successive orders the RMS values improve by a factor of two. For $N=6$ parameterization, RMS $\sim 0.01$ for $x$ close to $I$ and $x<0.8$, and RMS $\sim 0.1$ for $x \sim 0.9$. This indicates that for $x<0.8$ and $x \sim 1.0$, the shapes we obtain are close to true equilibrium shapes, but for $x \sim 0.9$, there are more uncertainties. By studying the change of RMS values at $x \sim 0.9$ for successive approximations, we find that the RMS values decrease very slowly in this region, much less than factors of two. This indicates that our model of a conducting drop using the equipotential surfaces of point charges is probably not good enough for $x \sim 0.9$. A more general or appropriate family of shapes appears to be in demand here. Hence one should regard the calculated results in this region with great reservations.

Let us take the $N=6$ curve at its face value and examine its main features. As we follow the curve from $x=1$ toward small $x$ values, the equilibrium shape elongates from a sphere, i.e., $R_{M A X} / R$ 
increases with decreasing $x$ in the region near $x=1$. This is in contrast to cases of small $x$ values $(x \lesssim 0.7)$ where $R_{\text {MAX }} / R$ is slowly decreasing with decreasing values of $x$. The shapes in the latter cases are long and look like a dumbbell. (see also Fig. 17) Similar to a volume charged drop there is a rapid change of shape, but occurring at $\mathrm{x} \approx 0.9$ in the present case. Actually the curve for $\mathrm{R}_{\mathrm{MAX}} / \mathrm{R}$ even turns back at $\mathrm{s}=0.887$ and again at $\mathrm{x}=0.906$. However it is in exactly this region that our results become unreliable and the double turn might be spurious (see Refs. 26 and 27 for a similar uncertainty which once existed in the volume charged case).

Let us now consider the nature of these equilibrium shapes by looking at the signs of the second derivatives of their energy with respect to all the parameters. The following results are found when we restrict the shapes to only the degrees of freedom that allow reflection symmetric shapes. For $1>x>0.887$ the energy of the drop is a maximum in one degree of freedom, but a minimum in the other $N-2$. Between the bends, for $0.887<x<0.906$, the energy is a minimum. For values of $\mathrm{x}$ smaller than 0.906 , it is again a maximum in one degree of freedom. With respect to the degrees of freedom that describe reflection asymmetric deformation, the energy of the drop is a minimum from $x=1$ to $x=0.892$. From $x=0.892$ to $x=0.68$ it is a maximum in one degree of freedom. Below $x=0.68$ it appears to be a maximum in two degrees of freedom. The implication of the change of the number of degrees of freedom with respect to which an equilibrium shape has a maximum energy is part of a general problem of the trend of 
equilibrium shapes as a function of a parameter $x$, which has been discussed by various authors $26,28,29)$ : One may classify the equilibrium shapes into three types. The first is a minimum, i.e., the system is stable in all directions. The second is a saddle point, at which the system is unstable in only one direction, i.e., it is a maximum in this direction. Physically this corresponds to a pass in a mountain range. The system has to go over the saddle point to get from one side of the range to the other. The third is what we shall call a "mountain top", at which the system is unstable in two or more directions. Thus in a subspace containing these directions this equilibrium point appears as a mountain top. Looking at the equilibrium shapes we have obtained with reference to both the symmetric and asymmetric degrees of freedom, we can distinguish the various types. The equilibrium point is a saddle from $x=1$ to $x=0.892$. From $x=0.892$ to $x=0.887$ it is a mountain top. Between the bends at $x=0.887$ and $x=0.906$ it is again a saddle. For $x$ smaller than 0.906 , it turns out to be a mountain top. As discussed before the saddle point close to $x=1$ is fairly well determined, but at the bends the results are no longer reliable.

In Fig. 18 we show the energy of the symmetric equilibrium shapes above that of a spherical liquid drop. It has an overall trend of an increase with a decrease of $x$, but it also exhibits kinks corresponding to the region of a bend shown in Fig. 16. In Fig. 17 we display several shapes along the $\mathbb{N}=6$ curve. Their RMS values are also indicated. 
4.G. Summary and Conclusions

We have looked at the static properties of a charged conducting drop and compared them with a volume charged drop. We have discussed the similarities as well as some of the differences. The symmetric equilibrium shapes of a conducting drop are determined with reasonable confidence for $x$ values not in the neighbourhood of $x=0.9$. At $x$ close to 0.9 the shapes found may not approximate the true equilibrium shapes adequately. The next step would be to try to use another parameterisation (e.g. that introduced by $\mathrm{Nix}^{18}$ ) so that equilibrium shapes at these values of $x$ are determined with greater reliability. This is important because it is in this region that we find interesting stability features, such as the occurrence of a bend in the family of equilibrium shapes and of points at which there is a change in the number of degrees of freedom with respect to which the shape has a maximum energy.

It is interesting to note that even some eighty years after Lord Rayleigh's study of a charged conducting drop, the whole problem is still a very open subject. We have been able to determine the saddle points of a charged conducting drop for values of $\mathrm{x}$ between 1 and 0.892 . But for the region of $x$ from zero up to 0.892 , one is still very ignorant of the saddle point shapes and energies of a charged conducting drop. 
5. On the Synthesis of the Liquid Drop Model and the Nilsson Single Particle Model.

With Applications to the Study of Shape Isomers and the stability of Superheavy Nuclei

\section{A. Introduction}

In the first three parts of the thesis we have referred, from several angles, to the idea of synthesizing a macroscopic and a microscopic model. In this part, which represents joint work with the authors quoted on $\mathrm{p}$. 4, we shall attack the problem directly and study in detail the synthesis of the liquid drop model ${ }^{1,4}$ and the Nilsson single particle $\operatorname{mode1} 1^{2,3)}$.

We shall begin by describing the Nilsson model on which the microscopic calculation is based, and also a calculation of the most important residual interaction that is not included in the model. This residual interaction is the pairing force ${ }^{30}$ ) which is responsible for the familiar odd-even mass differences. The description will be very brief both because it is not directly relevant to the main theme of this work and also because the materials have already been published ${ }^{31)}$. Details of the single particle calculations may be found in these references. In a similar manner the liquid drop mass formula due to Myers and Swiatecki ${ }^{4}$ is briefly described. Then the method of the synthesis of these two models is discussed in detail. The unified model is applied to give nuclear masses and deformations with very good agreement with experimental values. The calculations suggest the existence of metastable states of nuclei that correspond to nuclear 
shapes different from the ground state shapes. These shape isomers 32 ) are believed to be associated with the experimentally studied spontaneous fission isomers that occur in the actinide region. Some comparisons between theory and experiments are made. We next turn to the study of superheavy nuclei in the neighbourhood of $Z=114$ and $N=184-196$ which are predicted to be relatively stable 33,34$)$. Half-lives of alpha decay and spontaneous fission as well as stability against beta decay are calculated for the actinide elements as well as for these superheavy nuclei. By these quantitative studies we find that these superheavy nuclei could have very long total half lives. Several of them might even have life times comparable to the age of the solar system. A discussion is given of their possible production and of the most. favorable candidate for survival in earthly matter and in primary cosmic radiation ${ }^{34}$. 5.B. Single Particle Calculations The single particle calculations ${ }^{31}$ ) are based on the Nilsson model which assumes that the neutrons or protons move in a harmonic oscillator potential whose shape is described by two deformation parameters $\epsilon$ and $\epsilon_{4}$. The parameter $\epsilon$ describes a spheroidal deformation and the parameter $\epsilon_{4}$ describes a necking-in or bulging-out near the waist of the spheroids. Only axially symmetric and reflection symmetric shapes have been considered. The shapes in the $\left(\epsilon, \epsilon_{4}\right)$ plane are shown in Fig. 19. The relation of $\epsilon$ and $\epsilon_{4}$ with the commonly employed deformation parameters, $\alpha_{2}$ and $\alpha_{4}$ given by

It is seen in the figure that the shapes for $\epsilon_{4} \neq 0$ is too "rectangular" and is probably not good enough a description of the real nucleus. 
$R=R_{0}\left(1+\alpha_{2} P_{2}+\alpha_{4} P_{4}+\cdots\right)$ is shown in Fig. 20. The usual modification of the harmonic oscillator potential by the addition of a spin-orbit. coupling $(\underset{\sim}{\ell} \cdot \underline{s})$ is employed. A further modification is made such that effectively the central part of the harmonic oscillator, which is originally a parabolic shape, is flattened to some extent. Two parameters $\kappa$ and $\mu$ are associated with these two modifications and they are adjusted to reproduce experimental energy level spectra.

The Nilsson model has been applied with great success to the known nuclei. However one may question its reliability when one tries to apply it, in extrapolation, to very heavy nuclei far beyond the presently known region. One may suggest that a Hartree-Fock calculation based on detailed knowledge of nuclear forces may be more reliable. But the large number of matrix elements associated with the interactions anong the great number of particles involved makes such a calculation impractical with presently available computers. A more realistic one-bodycentral potential than the Nilsson potential described above is the Woods-Saxon potential ${ }^{36)}$ shape with a constant surface diffuseness. Several groups are currently studying this potential. So far this problem has not yet been adequately solved for strong deformations. On the other hand the Nilsson model has been studied for rather large. deformations and is fairly well understood. Thus we consider the Nilsson model to be the best available microscopic approach for our purpose of calculating the nuclear binding energy.

The two shell parametes $\kappa$ and $\mu$ have been adjusted by Gustafson et al. ${ }^{3)}$ to approximately reproduce the experimental level 
schemes for the rare earth nuclei $(A \sim 165)$ and the actinides $(A \sim 242)$. We take these values and for the other regions we assume $\kappa$ and $\mu$ to vary linearly with $A$. The parameters used are tabulated in Table 2. The results of the calculations reproduce the known magic numbers. For the $\mathrm{A} \sim 300$ region, we find the proton number 114 to be a fairly good magic number, confirming previous results 33 ). In this region we find, besides the magic neutron number $1.8 l_{1}$ that is genera.l. $y$ expected, also the magic neutron number ${ }^{+}$196. These results are shown in Figs. 21 and 22, which also shows the level schemes obtained by Rost ${ }^{35)}$ who used a spherical Woods-Saxon potential ${ }^{36)}$. Although in detail considerable differences are found, there is an overall agreement in the prediction of low level density for spherical shapes for $\mathrm{Z}=114-126$ and for $\mathrm{N}=178-184$. We show for illustration in Fig. 23 the Nilsson diagram for protons in the A 298 region. A gap exists in the level density for the spherical nucleus at $\mathrm{z}=114$ which is a proton shell. At each particular deformation, the potential energy can be found by filling up the levels with nucleons. The energy of the highest level that is filled is called the Fermi energy. 5.C. The Pairing Force

The average interaction among the nucleons has been represented by the Nilsson potential. The most important residual interaction is the pairing force, which is responsible for the occurrence of odd-even mass differences. This force was originally introduced by Bohr, Mottelson, and Pines ${ }^{30}$ ) and may be basically thought of as being a simplified representation of a $\delta$-force interaction. It is limited to 
act only between pairs of time reversed states, which have complete orbital overlap. Since protons and neutrons have different orbitals in general, the pairing force is assumed not to act between them. It is also assumed not to act in levels far below the Fermi energy, since interactions in these levels are much hindered because of the exclusion principle and the fact that neighbouring levels are ali occupied. This latter point is discussed in detail in Ref. 37.

The effect of the presence of neutrons (or protons) on the pairing interaction of the other kind of nucleons is represented by assuming a $(\mathbb{N}-Z) / A$ dependence of the pairing strengths. Furthermore there are indications in both theory 38,40) and experiments 39 ) that the pairing effect increases with increasing surface area of the nucleus. We thus follow stepien and Szymański ${ }^{40)}$ in assuming that the pairing strengths are proportional to the surface area. The choice of these strengths and the number of levels near the Fermi surface where the pairing force is assumed to act, is made so that the odd-even mass differences of the rare earth and actinide nuclei and their general $A^{-\frac{1}{2}}$ dependence are approximately reproduced ${ }^{31}$ ).

The effect of the inclusion of the pairing interaction relative to a simple summation of single-particle energies is exhibited in Fig. 24 for the case of ${ }^{254} \mathrm{Fm}$. The pairing effect increases the binding for all deformation. Though the increase in binding is in general not independent of $\epsilon$, it does not significantly change the equilibrium deformations from the values given by the Nilsson calculations without pairing. 
5.D. The Liquid Drop Formula

We have discussed in some detail in Part II of this thesis the potential energy formula of a leptodermous system, which is usually referred to as the liquid drop formula. We shall not repeat the discussions here but merely state below the liquid drop formula due to Myers and Swiatecki ${ }^{4}$. We have chosen this particular formula, because it involves only a few parameters which have been chosen to reproduce both the ground state masses and the spontaneous fission barriers. Thus the formula has been adjusted for large deformations that correspond to these fission barriers. This is most important for our purpose of calculating binding energies as a function of deformations.

The formula is given by

$$
E_{I D}=-a_{1}\left(1-k^{\prime} I^{2}\right) A+a_{2}\left(1-k^{\prime} I^{2}\right) A^{2 / 3} f(\text { shape })+E_{c},
$$

where $I=(N-Z) / A$ and $f$ (shape) is proportional to the nuclear surface area, having the value of 1 when the nucleus is spherical. The Coulomb energy $E_{c}$ is calculated by assuming the charge to be uniformly distributed in the nuclear volume. Surface diffuseness and exchange energy corrections to the Coulomb energy are also considered. The parameters in the above formula are given by Ref. 41. Note in particular that the same coefficient $\kappa^{\prime}$. is assumed for both the volume and the surface symmetry energies. 5.E. Generalised Strutinski Prescription and the Synthesis of the Liquid Drop Model and the Nilsson Model

In this section we shall study the prescription by which the synthesis of a microscopic and a macroscopic model is effected. As a 
preliminary, we shall give a discussion of the basis for such a synthesis. Though we have commented on it in the previous parts in some general terms, we shall now discuss the basis more specifically with reference to the NiIsson model and the liquid drop model.

5.E.1. The basis for the synthesis

In the Nilsson model, the nuclear potential energy may be written as the sum of single particle energies of nucleons filling up the Nilsson energy levels. It is well known that such a simple summation of single particle energies of the Nilsson potential is inadequate in the study of binding energies. In particular one is unable to account for the observation that the separation energy and the average binding energy are equal ${ }^{42}$ ). In Fig. 25 we display a potential energy surface for the nucleus ${ }^{252} \mathrm{Fm}$ as a function of deformation parameters $\epsilon$ and $\epsilon_{4}$ based on the simple summation procedure. It is seen that the energy gets larger and larger for large $\epsilon$ and large $\epsilon_{4}$. This is in disagreement with experiment since we know that the fission barrier of ${ }^{252} \mathrm{Fm}$ is only three or four MeV. This is not unexpected since we do not expect the Nilsson model to give correctly the absolute values of the binding energy as a function of deformation and mass number. However we find that it gives the relative values for neighbouring nuclei very well. On the other hand we have discussed in Part II that a liquid drop formula should reproduce the smooth trends and absolute values. The success of the liquid drop model as applied to fission phenomenon, where large deformations are involved and to the calculation of nuclear masses, where large number of nucleons are 
considered seems to confirm this. All this leads to the basic idea advocated by Myers and Swiatecki ${ }^{4}$, strutinski ${ }^{5}$ ) among others that if we take away the average trend from the single particle and pairing energies and replace it by the liquid drop formula, we would get a much improved potential energy surface, where the local wiggles are given by the Nilsson model calculations and the smooth trends are given by the liquid drop formula. In other words in the formula of the potential energy PE given by the sum of the single particle energies ${ }^{\mathrm{E}} \mathrm{SP}$

$$
\begin{aligned}
\mathrm{PE} & =\mathrm{E}_{\mathrm{SP}} \\
& =\left(\mathrm{E}_{\mathrm{SP}}-\overline{\mathrm{E}_{\mathrm{SP}}}\right)+\overline{\mathrm{E}_{\mathrm{SP}}},
\end{aligned}
$$

we replace the average single particle energy sum $\overline{\mathrm{E}_{\mathrm{SP}}}$ by the liquid. drop energy $E_{L D}$ :

$$
P E=\left(E_{S P}-\overline{E_{S P}}\right)+E_{I D}
$$

We may write out $\mathrm{E}_{\mathrm{SP}}$ in terms of the shell energy and the pairing energy of the neutrons and protons. Then,

$$
\begin{aligned}
& \operatorname{PE}\left(N, Z, \epsilon, \epsilon_{4}\right) \\
& =E_{L D}+E_{\text {Shell }}(N)+E_{\text {Pair }}(N)+E_{\text {Shell }}(Z)+E_{\text {Pair }}(Z),
\end{aligned}
$$

where $\mathrm{E}_{\text {Shell }}$ and. $\mathrm{E}_{\text {Pair }}$ are the shell and pairing corrections obtained by subtracting from the shell energy and pairing energy their average values. 
The smooth trend of the pairing energy turns out to be approximately a constant independent of $A$ and is equal to 31 ) $-2.3 \mathrm{MeV}$ which is conveniently subtracted off from the pairing energies. The smooth trend of the shell energy is however not a constant as a function of the mass number or of deformations. Its extraction is the key to the synthesis of the liquid drop and the Nilsson model. Once the smooth average of the shell energy is found, we can obtain the shell correction by subtracting it from the shell energy. The shell correction and the pairing correction are then added to the liquid drop energy to give the total potential energy, which has the useful features of both the microscopic and macroscopic approaches. The crucial problem of the extraction of the smooth average trend of the shell energy is discussed in the next subsection.

\section{E.2. Generalised Strutinski prescription}

In this subsection we shall study and generalise a prescription due to Strutinski for finding the smooth trend of the shell energy. The method is very similar to the method of data smoothing where one tries to obtain the average value at a point by evaluating a weighted mean of a region around that point.

Strutinski ${ }^{5}$ ) introduced a method to average over the energy levels rather than over the total energy itself. Given a level density $G(e)$ we may write the total shell energy as

$$
E(G)=\int^{E_{F}} 2 e G(e) d e
$$


where $\mathrm{E}_{F}$ is the Fermi energy. The factor 2 comes from the fact that there are two nucleons per level. The function $G(e)$ has the characteristic that it has both a smooth trend with a characteristic length $I$ which should be of the order of the Fermi energy and also short range fluctuations whose wavelengths are less than or equal to the energy spacing $\lambda$ between shells. The problem is to find a smooth level density $\mathrm{g}(\mathrm{e})$ which retains the long range variations but removes the short range oscillations (the shells).

One may formulate the problem by writing $G$ as follows

$$
G(e)=G_{L}(e)+G_{S}(e),
$$

where $G_{I}(e)$ is the slowly varying part and $G_{S}$ is the rapidly fluctuating part. Strutinski suggested that to smooth out $G_{S}(e)$ one could find an average by weighting the points by a Gaussian of suitable width. As already recognized by Strutinski, a simple Gaussian weighting, turns out to be inadequate because while it smooths out the rapidly fluctuating part, it also distorts the slowly varying part. In order to preserve the latter (i.e., $G_{L}(e)$ ) one introduces a correction factor $F$. Then the weighting is given by

$$
\frac{1}{r(\pi)^{\frac{1}{2}}} e^{-u^{2}} F(u) ; u=\frac{e-e^{1}}{r},
$$

where $r$ is the width of the Gaussian. The requirement on $F$ may be written as 


$$
\int_{-\infty}^{\infty} \frac{1}{r(\pi)^{\frac{1}{2}}} e^{-u^{2}} F(u) G(e) d e=G_{L}\left(e^{\prime}\right),
$$

which is equivalent to the two relations

$$
\int_{-\infty}^{\infty} \frac{1}{\gamma(\pi)^{\frac{1}{2}}} e^{-u^{2}} F(u) G_{L}(e) d e=G_{L}\left(e^{\prime}\right)
$$

and

$$
\int_{-\infty}^{\infty} \frac{1}{r(\pi)^{\frac{1}{2}}} e^{-u^{2}} F(u) G_{S}(e) d e=0 .
$$

In the case that $G_{L}$ is a finite polynomial of order $p, F$ can be found explicitly. Equation (21) gives

$$
\frac{1}{(\pi)^{\frac{1}{2}}} \int_{-\infty}^{\infty} u^{n} e^{-u^{2}} F(u) d u=\delta_{n, 0} ; 0 \leq n \leq p .
$$

Setting $F(u)$ to be a polynomial

$$
F(u)=\sum_{i} c_{i} u^{i}
$$

we find, after some work, from the last equation

$$
\begin{array}{ll}
c_{i}=0 & \text { for all } i>p \\
c_{i}=0 & \text { for all odd } i
\end{array}
$$


and

$$
\sum_{i=\text { even }} c_{i} \frac{(n+i-1) ! !}{2^{(n+i) / 2}}=\delta_{n, 0}
$$

which can be solved for the coefficients $C_{i}$. In other words, if $G_{L}$ is a polynomial of order $p$, we find a polynomial for $F(u)$ also of order of $p$ with only terms of even orders, such that with our weighting function, $G_{L}$ is retained in the smoothing procedure. When the polynomial for $F(u)$ is of order $m<p$, denoted by $F_{m}(u)$ we do not retain $G_{L}$ completely and the error made can be written down in general. The error ${ }^{\xi} \mathrm{I}$ made when $\mathrm{m}=\mathrm{p}-2$ and the coefficient of the $p^{\text {th }}$ order term in $G_{I}$ is $a_{p}$ turns out to be

$$
\xi_{L}=\left(\frac{r}{I}\right)^{m+2} a_{m+2} I_{m}^{m+2}
$$

where

$$
\begin{aligned}
& I_{m}^{m+2}=\frac{1}{(\pi)^{\frac{1}{2}}} \int_{-\infty}^{\infty} u^{m+2} e^{-u^{2}} F_{m}(u) d u \\
& \quad=(-)^{m / 2} \sum_{i=m / 2}^{m} \frac{m+i+1}{2^{i+1}} \frac{i !}{\left(i-\frac{m}{2}\right) !} .
\end{aligned}
$$

When $m$ is given, $\xi_{I}$ would be small when $r$ is small compared with L.

The prescription is also supposed to smooth out the shortrange fluctuating part of the level density, $G_{S}(e)$. Let us represent 
the term in $G_{S}(e)$ with the longest wavelength. $\lambda$ by

$$
G_{S}(e)=b \exp (i e / \lambda)
$$

If this term is smoothed away, terms of shorter wavelength are also smoothed away (see below Fq. 24). The error introduced by our prescription with $\mathrm{F}_{\mathrm{m}}(u)$ of the order $m$ is then given by

$$
\xi_{S}=\int_{-\infty}^{\infty} \cdot \frac{1}{\gamma(\pi)^{\frac{1}{2}}} e^{-u^{2}} F_{m}(u) G_{S}(e) d e .
$$

After some manipulation, we find

$$
\xi_{S}=G_{S}(e) e^{-(\gamma / 2 \lambda)^{2}} \sum_{k=0, \text { even }}^{m}\left(\frac{\gamma}{2 \lambda}\right)^{k} \frac{1}{\left(\frac{k}{2}\right) !}
$$

When we put $m$ to infinity the summation is just $\exp (r / 2 \lambda)^{2}$ and $\xi_{S}=G_{S}$, i.e., we do not smooth away $G_{S}$ at all. It is also clear that the smoothing is more effective when $\gamma$ is large compared with $\lambda$.

We sketch in Fig. 26 the total error $\left|\xi_{L}\right|+\left|\xi_{S}\right|$ as a function of $r$ for the cases of $m=0,6$, and $\infty$. The term $\xi_{I_{1}}$ dominates at large $r$ values and decreases as $m$ is increased. The term ${ }_{\mathrm{S}}$ dominates at small $r$ values. When $r=0, \xi_{S}$ is maximum, i.e., no smoothing has been done, but as $r$ increases ${ }_{\mathrm{S}}$ decreases. The spread of $\xi_{\mathrm{S}}$ is dependent on the values of $\mathrm{m}$. It is larger for larger $m$ and in the limit when $m$ is infinite, ${ }^{\xi}{ }_{S}$ has an infinite spread and no smoothing is made for any value of $r$. From the figure we see 
two points. First that we should use an $m$ value which is not too large (for which cases $\xi_{\mathrm{S}}$ is spread over all values of $\gamma$ ), and also, which is not too small (for which cases $\xi_{\mathrm{I}}$ is large even for $r$ values close to $2 \lambda$ ). Second, one should choose a $r$ value between $2 \lambda$ and $I$ such that the total error is a minimum. For an appropriately chosen $m$ value $(m=6$ in the figure) there actually exists a flat region inside these limits where the total error is small and is independent of $r$. This is the case one should choose.

Now we apply the smoothing prescription to the results of the Nilsson calculation. The Nilsson calculation gives a series of sharp energy levels $e_{v}$, so that

$$
G(e)=\sum_{v} \delta\left(e-e_{v}\right) .
$$

Then for a prescription with given values of $m$ and $r$, the smoothed level density $g$ is given by

$$
\begin{aligned}
g\left(e^{\prime}\right) & =\int_{-\infty}^{\infty} \frac{1}{\gamma(\pi)^{\frac{1}{2}}} e^{-u^{2}} F_{m}(u) G(e) d e \\
& =\int_{-\infty}^{\infty} \frac{1}{\gamma(\pi)^{\frac{1}{3}}} e^{-u^{2}} F_{m}(u) \sum_{v}\left(e-e_{v}\right) d e \\
& =\frac{1}{r(u)^{\frac{1}{3}}} \sum_{v} F_{m}\left(u_{v}\right) \exp \left(-u_{v}^{2}\right)
\end{aligned}
$$


where

$$
u_{v}=\frac{e^{\prime}-e_{v}}{r} .
$$

For the order $m$ to be seven ${ }^{+}$

$$
\begin{aligned}
F_{m}\left(u_{v}\right)=1 & +\left(\frac{1}{2}-u_{v}\right)^{2}+\left(\frac{3}{8}-\frac{3}{2} u_{v}^{2}+\frac{1}{2} u_{v}{ }^{4}\right) \\
& +\left(\frac{5}{16}-\frac{15}{8} u_{v}^{2}+\frac{5}{4} u_{v}^{4}-\frac{1}{6} u_{v}^{6}\right)+\cdots .
\end{aligned}
$$

Then $g(e)$ will have the same smooth polynomial behavior as $G(e)$ up to the seventh order. Any error will be in the eighth order.

The smooth total single particle energy is then

$$
E(g)=\int^{E_{F}} 2 e g(e) d e
$$

with the Fermi energy $E_{F}$ given by

+ R. A. Miller ${ }^{43}$ has done calculations along similar lines and written down $F$ as derivatives of $g(e)$. He has tried to study the convergence of the results of smoothing as a function of $\mathrm{m}$. However he used only one value of $r$ close to $2 \lambda$ (see Fig. 26) and so reached the wrong conclusion that the results of the prescription do not converge. 


$$
N=\int^{E_{F}} 2 g(e) d e,
$$

where $\mathbb{N}$ is the neutron number. Similar equations can be written down for the protons. The shell correction $E_{\text {Shell }}$ is then given by

$$
\mathrm{E}_{\text {Shell }}=\mathrm{E}(\mathrm{G})-\mathrm{E}(\mathrm{g}) \cdot
$$

In Figs. 27 and 28, we show this quantity as a function of the Gaussian width $r$ for the neutrons in the case of ${ }^{242} \mathrm{Pu}$ and ${ }^{208} \mathrm{~Pb}$, respectively. Similar figures are valid for the protons. It is obvious that if we use $F_{m}$ with $m=2$, we have a serious folding error and the result is strongly dependent on $r$, but when we use $m=6$, the result is rather $\gamma$-independent except when $\gamma$ is too large or too small. For a fixed value of $r$ (say $r=0.8$ hwo in the figure, where $w_{0}$ is the oscillator frequency in the Nilsson potential), the change from the zeroth $(\mathrm{m}=0)$ to the second order $(\mathrm{m}=2)$ is about $60 \mathrm{MeV}$; from second to fourth is I MeV, and from fourth to sixth only $\frac{1}{60} \mathrm{MeV}$. It is interesting to note that $(r / \mathrm{L})^{2}$ turns out to be just of this order $\frac{1}{60}$ and the above rate of convergence is indeed to be expected when the main error comes from $\xi_{\mathrm{L}}(\mathrm{Eq} \cdot 23)$. In our calculation we have used $m=6$ and $r=1.2 \hbar_{0}$ and we find that our results converge very well to a unique value for the shell correction. It is these shell wiggles that are added to the smooth liquid arop energy. 
In summary, we have thus a unified model obtained by replacing the smooth part of the total potential energy surface of the Nilsson model by the results of the liquid drop model. All local shell structure variations (the local wiggles of the energy surface) have, however, been retained.

5.F. Comparison with Experiments

To study the behavior of a nucleus at various deformations we have applied the unified model to calculate the total potential energy surface for the range of $\epsilon$ between -0.5 and 0.95 and $\epsilon_{4}$ between -0.08 and 0.16 . Smaller ranges of $\epsilon$ and $\epsilon_{4}$ are taken for some nuclei whose physically interesting features appear to be in a smaller region.

The lowest minimum in this potential energy surface corresponds to the ground state of the nucleus. Hence the ground state mass (or binding energy) and distortion can be read off from the energy surface and compared with experimental values. 
5.F.1. Nuclear massest

In Fig. 29, we compare empirical and theoretical masses with reference to the liquid drop masses at zero deformation. Thus the top curve gives the experimental values minus the respective spherical liquid drop masses in MeV. Immediately below, the theoretical values at ground state equilibrium deformations are plotted. These contain all the effects of distortions and shell structure. The differences between the theoretical and experimental values are exhibited as the third and lowermost graph in the figure. They reflect on the appropriateness both of the liquid drop parameters chosen and of the nuclear shell and pairing fields employed. The comparison shows very good agreements. Discrepancies are only around $1.5 \mathrm{MeV}$. Three points of deviation may be pointed out:

+ our calculations of masses are similar to those reported by P. A. Seeger and R. C. Perisho, Los Alamos Scientific Laboratory Report, LA-3751, 1967, which provided part of the original stimulus for undertaking calculations described in this section. These authors neglected the $\mathrm{P}_{4}$ degree of freedom and in their fission calculations represented the liquid drop barrier by a cubic in $\epsilon$. (There is an error in the coefficient of their cubic term.) However, they allow for an adjustment of liquid drop parameters. Our inclusion of the $\mathrm{P}_{6}$ degree of freedom appears to improve the mass fit considerably. No adjustment of liquid drop parameters is made in the present work. 
(i) The overall trend seems to be toward too small theoretical masses at large $A$ values.

(ii) There appear to be relatively large discrepancies connected with the doubly closed shell of ${ }^{208} \mathrm{~Pb}$. The theoretical binding energy is underestimated by about $2 \mathrm{MeV}$ around $\mathrm{A}=208$.

(iii) For large A values there is a marked discrepancy in the isospin dependence within each band of isotopic masses. First of all, it would be desirable to readjust the MyersSwiatecki liquid drop parameters using our shell corrections. Masses of spherical and deformed nuclei could be affected differently. If we further assume different isospin dependence (symmetry energy coefficients) for the volume and surface energy terms, we would probably be able to improve on the theoretical results.

On the other hand the underestimate of binding near the doubly closed shell may reflect on the details of the single particle calculations. The pairing energy calculation described in section 5.C collapses near closed shells, whereas in fact there should still remain some pairing energy of the order of one $\mathrm{MeV}$ as can be brought out by a random-phase-approximation calculation ${ }^{44}$ ). The underestimate in binding for A between 190 and 200 may be associated with the neglect of the rotational asymmetry degree of freedom which is believed to play a role in this region.

The masses for the superheavy nuclei beyond the present experimental region show a broad shell structure at $\mathrm{Z}=1.14$ and $\mathrm{N}=184$ to 
196. This shell effect is not as strong as for the ${ }^{208} \mathrm{~Pb}$ shell, but it may be a bit underestimated as in the $\mathrm{Pb}$ region. As shown below, this shell is the main reason to believe that there may exist in this region an island of relative stability which might be explored experimentally. 5.F.2. Ground state distortions

In Figs. 30 and 31 we exhibit theoretical deformation parameters $\epsilon$ and $\epsilon_{4}$ associated with nuclei in the rare earth and actinide regions. We should note that there is a general trend of the nuclear deformation to go from the spherical at one magic nucleus to a deformed nucleus with large $\epsilon$ but zero $\epsilon_{4}$, and then back to the spherical at the next magic nucleus. Nuclei in the intermediate region have nonzero values of $\epsilon_{4}$.

Let us comment here that if we look at the equilibrium $\epsilon$ calculated ${ }^{45}$ ) on the Nilsson model without renormalisation to the liquid drop smooth trends, we find the differences from our results to be small: in most cases less than five per cent. This is not unexpected because we know that the liquid drop part of the total potential energy is a smoothly varying function, always predicting ground states to be at zero deformation. Any deformed ground state would be due to the local fluctuations from the part connected with the single particle calculation, that have been retained. Hence both calculations are equally successful in giving the $\epsilon$ deformation.

In the case of $\epsilon_{4}$ deformations, let us look at the experimental results obtained by Hendrie et al. ${ }^{46}$ ), who did a detailed optical 
potential analysis of inelastic alpha scattering data on the rare earth nuclei. They assume the nuclear equipotential surfaces to be given by ${ }^{\dagger}$

$$
\mathrm{R}_{\frac{1}{2}}=\stackrel{\mathrm{Q}}{\mathrm{R}}_{\frac{1}{2}}\left(1+\beta_{2} \mathrm{Y}_{20}+\beta_{4} \mathrm{Y}_{40}+\beta_{6} \mathrm{Y}_{60}\right)
$$

The differential cross-section involving populations of rotational bands of even-even deformed nuclei up to $6^{+}$(in some cases $8^{+}$) state are fitted by a combination of $\beta_{2}, \beta_{4}$, and $\beta_{6}$. The experimental values of $\beta_{4}$ are compared with the theoretical values obtained from a transformation by means of Fig. 20. Thistt may be seen in Fig. 32. The agreement appears remarkable.

+ The relation between $\beta_{\lambda}$ and the conventional co-ordinate $\alpha_{\lambda}$ is given by

$$
\beta_{\lambda}=[4 \pi /(2 \lambda+1)]^{\frac{1}{2}} \alpha_{\lambda}
$$

†† The theoretical results in Fig. 32 represent an older calculation on a Nilsson model without renormalisation to the liquid drop smooth trends, but, as mentioned earlier, the new results are essentially the same within an accuracy of $5 \%$. 


\section{G. Potential Energy Surfaces}

From the minimum of a potential energy surface we can obtain the ground state mass and deformation which are discussed above. A further study of the potential energy surfaces will bring out more features of physical interest, in particular those connected with spontaneous fission barriers and shape isomers.

In Figs. 33a-l we exhibit the barriers obtained for isotopes of $Z=92$ to $Z=114$ as a function of $\epsilon$ with minimization of energy with respect of $\epsilon_{4}$ for each value of $\epsilon$. This type of plot represents a cut through the two-dimensional topographical map in the $\left(\epsilon, \epsilon_{4}\right)$ plane along the potential energy minimum path with the energies projected onto the $\epsilon$ axis.

In the following we shall study the structures found in the barriers with reference to these figures. The possible errors in these potential barriers are discussed at the end of this section.

\section{G.1. The structure of spontaneous fission barriers of}

\section{heavy and superheavy nuclei}

The conventional liquid drop barrier has the ordinary one peak shape, but because of secondary shell effects, structures can be found in the potential energy barrier. By the secondary shell effects, one refers to the extra shell binding that occurs at some moderate deformation as compared with the usually understood shell effects that appear for the spherical shapes. They were first pointed out by Geilikman 47) and studied by Myers and Swiatecki ${ }^{4}$ and Strutinski ${ }^{5}$ ). It was Strutinski 32) who first emphasized that they will cause a two-peaked fission barrier. 
As a general rule, for a nucleus with its proton or neutron number near a magic number, the ground state is spherical and the secondary shell effect occurs at $\epsilon \approx 0.4$. For a nucleus with its proton or neutron number away from a magic number, the ground state is at $\epsilon \approx 0.2$ and the secondary shell effect occurs at $\epsilon \approx 0.6$. In Fig. 34 we show the effect of shell corrections to the liquid drop barrier. It is seen that the fission barrier is basically that of the liquid drop with indentations due to shell effects. For the actinide region (A $\sim 242$ ), even though the liquid drop would like to have a spherical ground state minimum, the nucleus has deformation $\epsilon \approx 0.2$ because of shell effects. A secondary minimum occurs at $\epsilon \approx 0.6$ due to the secondary shell effect. For a nucleus near a closed shell, the shell correction makes the liquid drop minimum at the spherical shape even stronger and a secondary minimum is found at $\epsilon \approx 0.4$. For the actinides (and also the rare earth nuclei) another minimum corresponding to an oblate shape occurs (see Fig. 33). This minimum, for the actinide case, is usually more than $5 \mathrm{MeV}$ higher than the ground state. When the rotation asymmetric $(\gamma)$ degree of freedom is included, the nucleus corresponding to this minimum is found to be unstable in the $r$ direction ${ }^{48)}$, leading down to the lower ground state through a path provided by this extra degree of freedom. For the lighter nuclei in the rare earth region, this minimum is not much higher than the ground state minimum. In some cases it may actua.l.1y be lower and should be taken as the ground state. This oblate shape will then have important. 
physical significance, especially as regards the $N$ and $Z$ values where a transition from a prolate to an oblate ground state takes place.

As we have seen in Fig. 34, the existence of the two-peak structure of the potential energy barrier is due to the secondary shell effect. If the secondary shell effect occurs at or near the liquid drop saddle point, the two-peaked structure will be most prominent and the peaks will be of about equal height. If the secondary shell effect is to one side of the liquid drop barrier, the peak on this side will be smaller than on the other. In the extreme case when it is far off from the liquid drop saddle point we see a big peak and a very small second peak in the barrier. This is then essentially just the ordinary one peak barrier structure.

For the actinides the secondary shell effect occurs at $\epsilon \approx 0.60-0.70$. As we go from lighter to heavier actinides, the fissility parameter $\mathrm{x}$ increases and the liquid drop saddle points will move from large $\epsilon$ to small $\epsilon$. Thus the liquid drop saddle points for ${ }^{238} \mathrm{U}, \quad 242 \mathrm{Pu}, \quad 248 \mathrm{Cm}, \quad{ }^{250} \mathrm{Cf}$, and ${ }^{254} \mathrm{Fm}$ are at values of $\epsilon$ about $0.85,0.74,0.65,0.59$, and 0.54 , respectively. Then it appears that in the region around $\mathrm{Cm}$ the saddle points are at about

$\dagger$ The fissility parameter $x$ may be defined as

$$
x=\frac{Z^{2} / A}{50.88\left(1-1.7826 I^{2}\right)} ; I=\frac{\mathbb{N}-Z}{A},
$$

using the Myers-Swiatecki liquid drop parameters ${ }^{41)}$. 
the position of the secondary shell effect so that the two-peaked character of the barrier would be most prominent ${ }^{\dagger}$ with peaks of about equal height. As we go away from $\mathrm{cm}$ to nuclei with higher or lower values of $x$, one of the peaks will become smalier than the other and eventually it will be mostly washed out. Detailed results of actual calculations in our model may be found in Table 3 where we tabulate the heights of the two peaks as well as the secondary minimum in between, relative to the ground state. Because of the inaccuracy in the determination of the energy surface which will be discussed at the end of this section, as well as uncertainties in the assumptions explicitly and implicitly made in the present calculations, the table should be looked upon as an indication of trends rather than as a quantitative prediction. The trends are also illustrated in Fig. 35.

For the superheavy nuclei $(Z \sim 114$ and $\mathbb{N} \sim 184)$. Arguments similar to those above apply. Since there is practically no liguid arop barrier in this region, the two peak effect is apparent for ${ }^{294} 110$ where the secondary shell occurs at the flat part of the deformation curve, but is not apparent for cases where the secondary shell occurs at the rapidly dropping part (see Fig. 34 ).

It should be pointed out that for a particular element, a change in the number of neutrons may change the picture significantly.

+ This however does not mean that $\mathrm{Cm}$ isomers will have the longest spontaneous fission half lives, since these depend also on excitation energies of the isomeric state and the competition of gamma transition back into the ground state. 
Not only does $N$ affect the value of $x$, but shell structure effects associated with $N$ may have important consequences. An example is the following result from the present preliminary investigations. For $\mathrm{Z}$ between 102 and 114 and for $\mathbb{N}$ less than 176 , the nuclei have ground states near $\epsilon=0.3$, and secondary shell effects at $\epsilon \approx 0.70$. Since these nuclei have very large $x$ values the fission barriers exhibit only one peak. There is also a minimum at zero deformation which lies higher than the ground state. But as $N$ is increased, this minimum is getting lower until at $\mathbb{N} \sim 176-178$, it is actually lower than the minimum at $\epsilon \approx 0.3$ and so it has to be taken as the ground state. Hence for $\mathbb{N} \lesssim 176$ we have a deformed ground state with a onepeaked barrier. But for $N \geq 178$, we have a spherical ground state with a two-peaked barrier with the secondary minimum at $\epsilon \approx 0.3$. Obviously this latter case has a much thicker fission barrier and should be much more stable against spontaneous fission.

5.G.2. Spontaneous fission isomers in the actinide region The existence of the two-peaked structure with a secondary minimum in between may be associated with the spontaneous fission isomers that have been studied experimentally for some years. An isomeric state that corresponds to the secondary minimum has a different shape from the ground state and is higher than the ground state by several $\mathrm{MeV}$. The isomeric state may decay by gamma emission to the ground state or by spontaneous fission through the second barrier ${ }^{49)}$. The transition to the ground state is hindered by the presence of the first peak. For the actinides the first peak is large 
so that gamma transition is greatly hindered and it is more likely for the isomeric state to penetrate through the second peak and undergo fission. Obviously the ground state has a much thicker barrier against fission. Thus though the ground state of ${ }^{242} \mathrm{Pu}$ has a spontaneous fission half life of the order of $10^{5}$ years, one expects that the isomeric state has a half life of the order of 1.00 nano-seconds only.

Experimentally this kind of isomeric state is found in nuclei with $236 \lesssim A \lesssim 246$. The first fission isomer, in ${ }^{242} \mathrm{Am}$, was discovered by Polikanov et a. ${ }^{50)}$ and by Flerov et al. 5l) with a fission half life of $14 \mathrm{~ms}$. Since then a number of other cases have

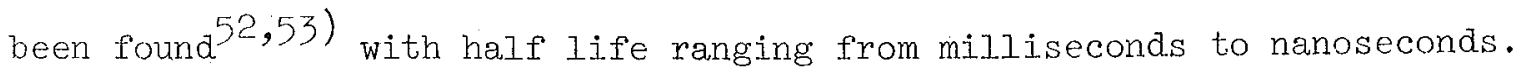
The isotope ${ }^{242} \mathrm{Am}$ seems to have an extraordinary long isomeric fission half life. This has been studied to some extent by Nix and Walker ${ }^{49}$ ) who also speculated about the possible explanations. The excitation energies of these isomeric states appear to lie between 2 and $4 \mathrm{MeV}$. Relevant data are shown in Table 4 together with our theoretical results taken from Table 3. As pointed out before, our theoretical values are not expected to be quantitative predictions, but rather an indication of the trends. Thus the discussion of trends in the last subsection is applicable here and can be used in a qualitative way to see where we expect to find these shape isomers.

Additional evidence that appears to support the existence of the secondary minimum is based on the study of the energy dependence of the thermal neutron fission cross sections for elements in the region $231 \leq \mathrm{A} \leq 242$. An example $i$ s the thermal neutron fission cross-section 
of ${ }^{235} \mathrm{U}$. Superposed on the fine structure of a few eV, occurring at about $6-7 \mathrm{MeV}$ of excitation, there appears a sequence of resonances with a spacing of about $100 \mathrm{eV}$ and a width of a few $\mathrm{eV}$. The ratio of the spacing of the resonance type states to the spacing of the usual type of states is about 500. It is about 50 for the case of ${ }^{24 I} \mathrm{Pu}$. If one interprets the resonance states as the states of the secondary minimum as suggested by Lynn ${ }^{54)}$, then, using the standard level density formula, one may estimate the secondary minimum to lie $1.5-3 \mathrm{MeV}$ above the ground state for the various nuclei between ${ }^{235} \mathrm{U}$ and ${ }^{242} \mathrm{Am}$. These results appear to be in qualitative agreement with the predictions of the present calculations.

\section{G.3. Shape isomers for the neutron deficient heavy} nuclei $(A \sim 206)$

Let us first make a comment about the shape isomers for the rare earth nuclei along the beta-stability line. These nuclei have their ground states situated at about $\epsilon=0.2$ and the secondary shell effect is expected to cause an indentation of the liquid drop barrier at $\epsilon \approx 0.6$. But since the liquid drop saddle point is at a much greater deformation than this value, what we expect to see is a two peak structure with the second peak much greater than the first peak. Actually the first peak is on the rising part of the liquid drop barrier so that its effect is further reduced. Then if the nucleus is at the isomeric state, it would probably prefer a gamma transition to the ground state rather than spontaneous fission through the second barrier. 
Recently Bjфrnholm ${ }^{55)}$ suggested that one might look for fission isomers in some neutron deficient heavy nuclei such as ${ }^{204} 86^{11}{ }^{118}$. This may be argued as follows. The proton and neutron numbers are close to the magic numbers 82 and 126 respectively. One thus expects the ground state to be spherical and the secondary shell effect to occur at $\epsilon=0.4$. Compared with the rare earth nuclei, this secondary shell effect occurs at a smaller deformation than for the rare earths so that its effect will be stronger. For the neutron deficient case the liquid drop fissility parameter is increased so that the liquid drop saddle point will be moved toward the point of secondary shell effect. As discussed in the subsection G.I. this would enhance the two-peak effect.

We have calculated the barriers for the neutron deficient heavy nuclei centered round $\mathrm{Z}=86, \mathrm{~A}=202$. The results are displayed in Figs. 36 a-e. They seem to indicate that while the above discussion is true, the suggested enhancement of the two-peak effect on the fission barrier is not enough. Even though the fissility parameter is increased somewhat, the liquid drop saddle point is still at a very large deformation. Thus the shell effects will occur at the rising part of this liquid drop barrier with the result that the isomeric state is at a very high excitation energy above the ground state ( $5 \mathrm{MeV}$ ). For the same reason the second peak is much broader than the first peak. Hence for these cases one expects the same conclusions as in the rare earths case, namely that if the nucleus is in the isomeric state, it would more likely undergo penetration through the first barrier and gamma decay to the ground state than spontaneous fission through the second barrier. 
In this study one realises an important point that when one is looking for regions of fission isomers, one should look at the shell effects on the background of the liquid drop fission barrier. Just looking at the shell corrections by themselves may be misleading.

5.G.4. Uncertainties in the potential energy surfaces

Two representative energy surfaces are exhibited in Figs. 37a,b. The separate contributions from the liquid drop terms and the shell plus pairing energies are exhibited in Figs. 38 and 39. For small distortions from the spherical shape we expect the $\left(\epsilon, \epsilon_{4}\right)$ parameterization as used to be adequate. However at large distortions higher multipoles will be important in the calculations of saddle point energies ${ }^{27)}$. Since for larger values of the fissility parameter $x$, the liquid drop saddle points occur at smaller distortions and vice versa, we expect that higher multipoles to be important for lighter nuclei whose values of $x$ are small, and that the $\left(\epsilon, \epsilon_{4}\right)$ parameterization should be sufficient for heavier nuclei which have large values of $x$. Thus when we compare the liquid drop saddle point energies on our $\left(\epsilon, \epsilon_{4}\right)$ scheme with the more general parameterization used by Cohen and Swiatecki ${ }^{27}$ ) we find that for $U$, with its saddle point at $\epsilon \approx 0.85$, our value is too high by $0.6 \mathrm{MeV}$; for $\mathrm{Pu}$ whose saddle point is at $\epsilon \approx 0.75$, our result is too high by $0.3 \mathrm{MeV}$; and for nuclei heavier than $\mathrm{Cm}$ $(z=96)$ the error is less than $0.1 \mathrm{MeV}$. In particular for superheavy nuclei $(Z \approx 1.14, A \approx 298)$, the error due to the restricted parameterization should be small. 
The potential energy surface plots show the importance of the $\epsilon_{4}$ degree of freedom as $\epsilon$. is increased. Although in the ground states both positive and negative values of $\epsilon_{4}$ occur, the saddle point always occurs for a positive $\epsilon_{4}$, representing a smaller waistline relative to the spheroid. In, Fig. 24, one may study the effect of the $\epsilon_{4}$ degree of freedom on the barrier of ${ }^{254} \mathrm{Fm}$.

The further considerations of deviations from axial symmetry of the nuclear shapes appear to reduce the saddle point energies. Thus as reported by V. V. Pashkevich ${ }^{56}$ the energies of the saddle points closest to the ground state for nuclei between ${ }^{240} \mathrm{Pu}$ and $256 \mathrm{Fm}$ are reduced by amounts ranging from $0.4 \mathrm{MeV}$ to $2.1 \mathrm{MeV}$.

On the whole we would say that we have over-estimated the potential surfaces somewhat at large deformations. At small deformations, they should be reasonably reliable.

5.H. Barrier Penetration and Spontaneous Fission Half Lives

For the purpose of calculating spontaneous fission half lives, we use the simple WKB theory for the penetration of a barrier. Let us assume that the problem is one-dimensional and that $\epsilon$ is the relevant fission co-ordinate. According to the WKB approximation, the probability for the penetration of a barrier is given by the expression

$$
P=\exp \left\{-2 \int_{\epsilon^{\prime}}^{\epsilon^{\prime \prime}}\left(\frac{2 B}{\hbar^{2}}(W(\epsilon)-E)\right)^{\frac{1}{2}} d \epsilon\right\} \equiv \exp (-K)
$$


where $B$ is the inertial mass associated with fission assumed to be independent of $\epsilon, E$ is the initial excitation energy of the nucleus towards fission, and $W(\epsilon)$ represents the barrier as obtained from a potential energy surface considered in the previous section. There exists an improved expression, as shown by P. O. Fröman and N. Fröman ${ }^{57)}$ :

$$
P=(1+\exp K)^{-1}
$$

This differs from the one above mainly for small $K$ values, i.e., for energies $E$ near the top of the fission barrier. In particular, when $\mathrm{E}$ is equal to the top of the barrier, the probability for penetration is 0.5 . In our calculations below we consider only very small $\mathrm{E}$ values for spontaneous fission, so we use the previous expression, which should be adequate. Since $\epsilon$ is dimensionless, $B$ will have the dimensions of a moment of inertia. Thus if we scale the nuclear system simply according to its mass number, the $B$ will be proportional to $\mathrm{A}^{5 / 3}$.

Let $n$ be the frequency of beta vibrational motion that is associated with the fission mode. Setting $n=10^{20.38}$ corresponding to a nominal vibrational energy of one MeV, we have the half life given by

$$
\tau=\frac{\ln 2}{\mathrm{n}} \frac{1}{\overline{\mathrm{p}}}=10^{-20.54} \exp \mathrm{K}(\text { seconds })
$$

or

$$
T=10^{-28.04} \exp \mathrm{K}(\text { years }) .
$$


Three main sources of error enter into the half life estimation. (1) We have made a simplification of the problem so that it is reduced to a one dimensional barrier penetration. We construct a path in the enexgy surface by minimizing the potential energy with respect to $\epsilon_{4}$ for each $\epsilon$ and then projecting this path onto the $\epsilon$ axis. (2) With the barrier, $W(\epsilon)$, thus obtained, there are errors for large $\epsilon$ since we consider only $\epsilon$ and $\epsilon_{4}$ deformations whereas higher orders of deformations are important at large distortions. This effect will be especially large for light actinides whose barriers extend to rather large distortions. (3) Furthermore, shell effects will have their main impact near the ground states and will be washed out at large distortions. Thus any error in shell calculations will distort the potential barrier and hence affect the life time estimates. All these errors will be very crudely accounted for when we treat $\mathrm{BA}^{-5 / 3}$ as a paraneter to be adjusted so that the experimental half lives are reproduced. We have estimated this parameter $\mathrm{BA}^{-5 / 3}$ by applying Eqs. 25 and 26 to the potential barriers we have calculated for the actinides $z=92-102$ and their experimental spontaneous fission half lives. We have attempted to see the dependence of this parameter on saddle point shapes by plotting it against the fissility parameter $x$ and also against the mean deformation of their barriers. In both cases, no simple trends are discerned. Thus as an assumption, we have taken $\mathrm{BA}^{-5 / 3}$ to be a constant for all heavy and superheavy nuclei. This is the simplest procedure one can take in lieu of anything definitely better, but one is not at all clear how valid this assumption is. 
Several methods are used to estimate $\mathrm{BA}^{-5 / 3}$. The first is taken from a microscopic calculation due to sobiczewski et al.7). The inertial parameters for the heavy nuclei are found to cluster within $30 \%$ of a mean value.

A second estimate of $\mathrm{BA}^{-5 / 3}$ is the empirical values obtained by using the barriers for the actinides which we found from our calculations and requiring these to give the correct experimental half lives. These are also found to cluster within r $30 \%$ about a mean value. A third estimate is also empirical and is due to Moretto and Swiatecki ${ }^{58)}$. They used liquid drop barriers modified by a Myers-Swiatecki shell correction term ${ }^{41}$ ) and with the ground state masses and fission barriers adjusted to experimental values. They are able to estimate the mean value of $\mathrm{BA}^{-5 / 3}$ for the actinides with only a $10 \%$ spread. It was found that all of these three estimates lie within 30\% of each other.

$$
\text { These estimates of the mean value of } \mathrm{BA}^{-5 / 3} \text { are shown in }
$$

Fig. 40, where we have plotted

$$
\text { en } T \text { against }\left(B A^{-5 / 3} / \pi^{2}\right)^{\frac{1}{2}}
$$

for $z=110$ and 114. The siope in this plot is

$$
2 \sqrt{2} A^{5 / 6} \int(W(\epsilon)-E)^{\frac{1}{2}} d \epsilon
$$

where the excitation energy $\mathrm{E}$ is taken as half an $\mathrm{MeV}$, corresponding to the zero point vibrational energy in the fission mode. From this figure the half lives can be read off. We have taken among the three 
estimates, the Moretto-Swiatecki value which is the lowest of the three. The reason for the choice is that this estimate incorporates the experimental ground state masses and fission barriers, whereas the other estimates have uncertainties in both these quantities. If we had taken the other estimates, some of our values for spontaneous fission half lives would be larger by one or two orders of magnitude while others are increased by a factor less than 10 .

It is to be commented here that this inertial parameter which we adopt is more than seven times the value obtained by the assumption of a pure liquid drop with irrotational flow, which of course cannot be considered to be anything more than an extreme lower limit. This ratio, seven, turns out to be somewhat larger than the corresponding ratio for the rotational moment of inertia for deformed nuclei and also the ratio for the quadrupole vibration.

The spontaneous fission half lives of the superheavy nuclei are discussed in the next subsection. The half lives for the actinides are represented in Table 5 as the ratio in powers of ten of the experimenta]. to theoretical values. There seems to be a systematic underestimate of half lives on the neutron-poor side and an overestimation on the neutronrich side. A readjustment of the liquid drop parameters with independent volume and surface symmetry energy coefficients might be able to take care of this systematic discrepancy. 5.I. Stability of Superheavy Nuclei

There are three main mechanisms for the decay of a nucleus: spontaneous fission, alpha decay, and beta decay (or electron capture). 
Alpha decay half lives can be estimated from the Q-values of the process, which are directly found from the masses of parent and daughter nuclei. Similarly by comparing masses of adjacent isobars, beta stability can be determined. Since these processes involve only mass differences between nuclei one or two units of $\mathbb{N}$ or $Z$ from each other their half life estimations will be relatively little affected by any errors that occur in these nuclei to a similar degree. Hence the alpha and beta stabilities can be determined with reasonable reliability. For the actinide region (Table 5) we are able to reproduce the experimental Q-values of alpha decay to within $\pm 0.2 \mathrm{MeV}$ and beta stable nuclei are usually verified. The results for the lead region are not so satisfactory. In this region the differences between experimental and theoretical Q-values for alpha decay could be about $0.6 \mathrm{M} \in \mathrm{V}$. This is partly due to the inadequacy of our calculations in reproducing the trends of the nuclear masses near the lead region (see section F.I.).

The estimation of spontaneous fission half lives involves larger uncertainties as discussed in the last section.

5.I.1. Island of stability in the neighbourhood of $\mathrm{Z}=114$ and $\mathrm{N}=184-196$

The stability against alpha and beta decay as well as spontaneous fission has been worked out for nuclei with proton number from 106 to 128 and neutron numbers from 178 to 204. In this region are the magic numbers $\mathrm{Z}=114, \mathbb{N}=184$, and $N=196$ (see Figs. 21 and 2a). The results are tabulated in Tables 6-8, which are summarised in the half life contours of Fig. 4l. 
Some general features of this figure may be pointed out. The longest fission half-lives center rather symmetrically around $(z=174$, $\mathbb{N}=184-196)$. It must be emphasized here that any stability against spontaneous fission in this region is due to the extra binding resulting from the shell effect so that as one goes away from $z=114$ and $N=184-196$, the fission half-lives decrease rapidly. Without the shell effect, the alpha half-lives depend on the inclination of the $\Delta \mathbb{N}=\Delta Z$ line (which is the direction of alpha decay) with respect to the direction of the beta stability valley. The shell effect essentially increases the alpha half-lives for nuclei with $\mathrm{Z}<114$ and $\mathrm{N}<184$ and decreases those for nuclei $\mathrm{z}>114$ and $\mathrm{N}>184$ and also $Z>114$ and $N>196$. The kinks in the curves occur when either the parent or the daughter nucleus experiences a maximum shell binding effect.

The great uncertainty associated with the numbers obtained must be emphasized. First of all there is the uncertainty of the extrapolation of the shell model potential. to an unknown mass region. Furthermore, a deviation of $30 \%$ in the estimate of the inertia parameter $B$ corresponds roughly to a factor of $10^{6}$ in the spontaneous fission halflives, while a $1 \mathrm{MeV}$ deviation in alpha energy corresponds to a factor $10^{6}$ difference in alpha half-lives. An underestimate of a given nuclear mass due to a local shell effect leads normally to an overestimate of the fission half-life. On the other hand, the error in. alpha energy is comparatively small. For the actinide region (where we do not have the uncertainty due to the extrapolation of parameters, 
Table 5, our alpha energies are within 5\% of the experimental values corresponding to half-lives agreeing within a factor of ten, but our fission half-lives for some isotopes can be wrong by a factor as large as $10^{6}$ either. way.

All these uncertainties may move the contours of half-lives in Fig. 4I, but the general pattern should remain the same so long as $\mathrm{Z}=114, \mathbb{N}=184$, and $\mathrm{N}=196$ are good magic numbers. The magic numbers $Z=114$ and $N=184$ have been confirmed by various calculations $^{33)}$ so that the part of the figure around the nucleus 114298 can be used with reasonable confidence. The magic number $\mathbb{N}=196$, however, has not yet been verified by other calculations. At the moment one is not sure whether this number will remain magic when a more realistic calculation than ours is made. ${ }^{+}$

The use of this figure as a guide in the search for relatively long-lived superheavy nuclei will be illustrated in section $J$ below.

5.I.2. Possibility of the occurrence of surviving superheavy elements in nature

Applying a "survival-of-the-fittest" test with respect to fission, alpha decay, and beta decay in the above region and taking the calculated numbers at their face value, one ends up with one probable

+ Recently Bolsterli, Fiset, and $\mathrm{Nix}^{59)}$ calculated the single particle energies by a,scheme in which no extrapolation of parameters is necessary. Their preliminary results indicate that there is no gap in the levels at $\mathbb{N}=196$. 
candidate for survival in earthly matter, namely 294110 , which has a total half life of about $10^{8}$ years. However, the uncertainty of our numbers as discussed above may indicate that, instead, a nucleus closeby may have a better chance of survival.

Self-consistent field calculations of the electronic configurations 60,61 ) indicate that the elements with even $z$ from 106 up to 116 have chemical properties similar to those of $\mathrm{W}$, Os, $\mathrm{Pt}, \mathrm{Hg}, \mathrm{Pb}$, and Po, respectively (Fig. 42). So these superheavy elements, if they occur in nature, may be found in ores of their respective chemical homologues. However if the total half-life falls below $\sim 2 \times 10^{8}$ years, its detection in earthly matter is beyond the capabilities of our present techniques. ${ }^{\dagger}$

Even if the longest half life in this region of elements is less than $2 \times 10^{8}$ years, it may be possible to obtain information concerning superheavy nuclei existing at some time in the past by searching for neutron-rich products of spontaneous fission in meteorites or in natural ores of platinum and its neighbouring elements.

A question may be asked whether such a long-lived superheavy element may be produced in nature in the first place. This is still an open question. However, it may be th that such a superheavy element could be formed by the so-called $r$-process ${ }^{62)}$ in which a nucleus absorbs

t We would like to thank Dr. Iuciano Moretto for drawing our attention to this point.

H. We are grateful to Dr. P. A. Seeger for helpful discussions of the r-process. 
a large number of neutrons very rapidly and then undergoes successive beta decays, ending up as much heavier relatively stable nuclei. ${ }^{\dagger}$ Most very neutron rich isotopes seem to be sufficiently fission stable for this process, which also requires a condition of huge neutron flux and. very high temperatures. This condition may have prevailed at some point in the history of the universe and may also exist in some massive stars and quasi-stellar radio objects at this present time. This at once raises the possibility of detecting superheavy nuclei in the primary cosmic radiation. According to the most optimistic estimates, the nuclei of interest in the primary cosmic radiation may have been produced $10^{5}$ years ago, while elements in the solar system have an age of $\sim 510^{9}$ years. If we take Fig. 41 at its face value, we see that in the study of the primary cosmic rays, one might be able to find a few more nuclei which live longer than $10^{5}$ years.

$\dagger$ our estimate of masses along the prospective $r$-process path is, however, sensitive to the value assumed for the coefficient of the surface symmetry energy. Conceivably the value of this coefficient, after readjustment of all the liquid drop parameters, might be such as to make the generation of superheavy elements impossible. The isotopic trends of actinide fission half-lives, which we fail to reproduce adequately (Table 5), may be indicative of this. 
A recent preliminary search of element 110 in a platinum ore at Berkeley and Livermore has yielded negative results 63,64 ). A study of very heavy nuclei in the primary cosmic rays has recently been carried. out by P. H. Fowler, P. B. Price, and R. W. Walker in a balloon experiment. The data are still under analysis.

\section{J. Possible Experimental Production of Superheavy Nuclei}

The heaviest elements presently produced $(\mathrm{Z}>100)$ are all synthesized by the bombardment of target elements of sufficiently high atomic number with beams of heavy ions. The heaviest ion presently available is ${ }_{10} 8 \mathrm{Ar}$, but in the future ions as heavy as $92^{\mathrm{U}}$ may be accelerated. On the other hand there is also a possiblity of producing these superheavy nuclei by bombarding a target with an intense flux of neutrons in a reactor. These will be discussed below ${ }^{\dagger}$.

5.J.1. Heavy ion reactions by available projectiles

By heavy ion reactions one tends to reach nuclei on the neutron deficient side of the beta stability line. This is so because the stability line bends more and more towards the neutron-rich side relative to its initial $45^{\circ}$ direction in the $N-Z$ plane. Both target and projectile are therefore less neutron-rich than the center of the superheavy region $(Z=114, \quad N=184)$, near to which the stability line happens to pass.

$\dagger$ The reader is also referred to the extensive review given in Ref. 65 . 
One of the most neutron-rich targets is ${ }^{248} \mathrm{~cm}$ and the most neutron-rich projectile that is presently available is $40 \mathrm{Ar}$. In the experiments by Thompson et al. ${ }^{66)}$ and Ghiorso et al. ${ }^{67)}$, the following reaction was attempted:

$$
\begin{gathered}
248 \\
96
\end{gathered}+\begin{aligned}
& 40 \mathrm{Ar} \\
& 18
\end{aligned} \rightarrow{ }^{284} 114+4 \mathrm{n} .
$$

One obtains only the relatively light isotope ${ }^{284} 114$, whose half life we estimate to be much less than $10^{-15}$ seconds. This is beyond the sensitivity of the present experimental techniques. The unfortunate loss of four neutrons is necessary to take away the excess energy of the compound nucleus which results from the high energy reguired to overcome the Coulomb barrier between the heavy ion and the target nucleus. Even with a ${ }_{20}^{48} \mathrm{Ca}$ projectile,

$$
{ }_{20}^{48} \mathrm{Ca}+{ }_{94}^{244} \mathrm{Pu}={ }^{288} 114+4 \mathrm{n},
$$

the product ${ }^{288} 114$ has a half life less than $10^{-10}$ seconds. At the moment it appears from Fig. 41 that one has to obtain an isotope of 114 with mass number equal to or greater than 290 before the half life becomes long enough to make detection possible. For this $\vec{a}$ 'heavy projectile like ${ }_{36}^{86} \mathrm{Kr}$ is required. 
5.J.2. Heavy ion reactions by future projectiles

When heavier and hence more neutron-rich ions than $\mathrm{Ar}^{40}$ can be accelerated the prospect is much better for the production of the superheavy nuclei. In general one has to overshoot the $1.14^{298}$ nucleus and let various decay mechanisms take one to its neighbourhood. An extreme example is the reaction $U^{238}+U^{238}$. Either a transfer reaction takes place where the target takes off a part of the projectile or a compound nucleus is formed which then undergoes fission. One hopes to find products that are close enough to the center of the island of stability so that they have long enough life times to make detection possible.

An example that is not so extreme is furnished by reactions induced by the ${ }_{36}^{86} \mathrm{Kr}$ ion. In Table 9 , we show the compound nuclei that might be formed by bombarding various neutron rich targets from $\mathrm{Pb}$ to $\mathrm{Cm}$ with ${ }^{86} \mathrm{Kr}$. The question whether such a compound nucleus would be formed will be discussed below. At the moment, let us assume that by emitting four neutrons we get a cold compound nucleus in the ground state. If we look at Fig. 4I, we see that for ${ }^{208} \mathrm{~Pb}$ and ${ }^{210}$ Po targets, the compound nucleus undergoes spontaneous fission at once and we do not expect to produce any superheavy nuclei. With targets heavier than ${ }^{226} \mathrm{Ra}$, it appears that the alpha half life is always less than the spontaneous fission half life (Fig. 41). Indeed if we let the compound nucleus decay by emitting alpha particles all the way we end up in each case with a long-lived superheavy nucleus. 
The above discussion assumes that the compound nucleus was formed in the first place. This assumption is very questionable for the following reasons. (1) There are indications that for the same products, the cross-section of a reaction with a heavy projectile is cut down by several orders of magnitude compared with a reaction in whicha lighter projectile is used. (2) The large angular momentum introduced with the heavy projectile may cause the compound nucleus to fission at once. (3) Furthermore we know that any binding of a superheavy nucleus is due to a shell effect. At the excitation energy of the compound nucleus when it is first formed, the shell effect might be greatly reduced so that little binding would be present and the compound nucleus would break up (or would simply not be formed) before any de-excitation can take place by neutron or charged particle emission. The first two points are illustrated by the fact that the production ${ }^{68)}$ of ${ }^{260} 104$ by bombardment of 242 Pu with ${ }^{22}$ Ne has a cross-section of only $10^{-34} \mathrm{~cm}^{2}$. The last point is a difficulty characteristic of the production of superheavy nuclei.

These effects have not yet been understood and no definite opinion can be expressed as to their importance in any future attempts to produce superheavy nuclei by heavy ion reactions. Further studies of these problems are essential not only for the production of superheavy nuclei but also for an understanding of heavy ion reactions in general. 


\section{J.3. Neutron capture reactions}

An alternative way to attempt the production of superheavy nuclei is by exposing heavy nuclei to a high flux of neutrons. There is a competition between $(n, \gamma)$ and $(r, n)$ reactions. Under suitable conditions of extremely intense neutron flux and very high temperatures, the nuclei will capture a large number of neutrons and then beta decay, ending up as heavy nuclei of much higher proton number, which in turn undergo the same process. Eventually they would, hopefully, reach the superheavy region. An intense neutron flux can be found in a nuclear reactor and also in nuclear explosions 65 ). By the former, one can achieve a neutron flux of $10^{15} / \mathrm{cm}^{2}-$ sec. By the latter the neutron flux is much larger, of the order of $10^{31} / \mathrm{cm}^{2}-\mathrm{sec}$, but the exposure time is less than 1 usec. The advantage of both these methods is that comparatively large masses of target material can be used. However these methods have produced fewer heavy elements than expected. Indeed $257 \mathrm{Fm}$ is the nucleus with the largest $\mathrm{Z}$ and $\mathrm{A}$ numbers that has been made ${ }^{65}$ ). On our model we do not expect $258_{\mathrm{Fm}}$ to possess very much shorter life time than $257 \mathrm{Fm}$ or ${ }^{256} \mathrm{Fm}$. There are presently no satisfactory explanations as to why heavier nuclei are not produced. 5.K. Summary

In this part of the thesis we have tried to make use of the ideas advanced in the previous parts in a practical application in the synthesis of the Myers-Swiatecki liquid drop formula and the Nilsson single particle calculations. By means of a generalised strutinski 
prescription we have been able to replace the smooth average of the Nilsson calculations by the liguid drop (or leptodermous) model. The resulting unified model is expected to be good in accounting for not only single particle effects but also the absolute values and trends of the binding energies.

There are two important consequences that come out of this study. First of all is the occurrence of single particle structure fission barrier. In particular, one finds in the energy barrier a two-peak structure with a secondary minimum between the two peaks. This secondary minimum may be associated with spontaneous fission isomers found in the actinide region. We have given a discussion of the trends and the regions where one would expect to find observable shape isomers. The second conseguence are the predictions about superheavy elements. We have confirmed previous calculations that $\mathrm{Z}=114$ and $\mathbb{N}=184$ are magic numbers, but the present work suggests that $\mathbb{N}=196$ may also be magic. We have been able to make quantitative predictions on masses, deformations, as well as half lives for the various decay mechanisms for these superheavy nuclei. It is found that some of the half lives might be extremely long, even of the order of the age of the solar system. Though great uncertainties are involved in the numbers as discussed, one can still use these predictions as an indication of trends and as a general guide in attempts to produce superheavy nuclei or in a search for them in nature. 


\section{Acknowledgments}

I am greatly indebted to Wladyslaw J. Swiatecki for his patient guidance and ready assistance throughout my research. He not only suggested the problems studied in this thesis but also contributed numerous ideas and suggestions. He provided the main impetus for this work.

I am deeply grateful for the constant advice and most willing help of Sven Gösta Nilsson who directed my research in the last part of this thesis. His many ideas and untiring efforts have made our collaboration the most exciting period of my research experience.

Great appreciation is also expressed to StanleyG. Thompson for his encouragement and discussions. It is a privilege to work in cooperation with his experimental group, which provides a most stimulating atmosphere.

I am also very grateful to Glenn T. Seaborg for many discussions and for his great interest in the last part of this work, which is the main reason for its present line of development.

I have benefited immensely by numerous discussions with William D. Myers and J. Rayford Nix, and I would like to thank them for their help and advice on computer programming. Thanks are also due to Luciano G. Moretto and Eli Cheifetz for discussions on various aspects of this work.

The co-operation of Christer Gustafson, Inger-Lena Lamm, Peter Möller, Bjorn Nilsson, Adam Sobiczewski, Zdzislaw Szymański, and Slawomix Wycech is gratefully acknowledged.

The work was done under the auspices of the U.S. Atomic Energy Commission . 


\section{References}

1) Lise Meitner and O. R. Frisch, Nature $\underset{\sim}{143}$ (1939) 239; Niels Bohr and John Archibald Wheeler, Phys. Rev. 56 (1939) 426

2) S. G. Nilsson, Mat. Fys. Medd. Dan. Vid. Selsk. 29, No. 16 (1955)

3) C. Gustafson, I. I. Lamm, B. Nilsson, and S. G. Nilsson, Arkiv Fysik 36 (1967) 613

4) W. D. Myers and W. J. Swiatecki, Nuclear Physics 81 (1966) I

5) V. M. Strutinsky, Nuclear Physics 192 (1967) 420

6) David Lawrence Hill and John Archibald Wheeler, Phys. Rev. 89, (1953) 1102

7) A. Sobiczewski, Z. Szymański, S. Wycech, S. G. Nilsson, J. R. Nix, C. F. Tsang, C. Gustafson, P. Moller, and B. Nilsson, Microscopic Calculations of the Inertial Mass Parameter for Fissioning Nuclei, to be published in Nuclear Physics;

A. Sobiczewski, Z. Szymański, S. Wycech, S. G. Nilsson, J. R. Nix, C. F. Tsang, C. Gustafson, P. Moller, and B. Nilsson, Microscopic Values of Inertial Parameters of Heavy and Superheavy Nuclei (in Russian) to be published in Izv. Akad. Nauk. (USSR)

8) William D. Myers, Average Nuclear Properties (Ph.D. Thesis), Lawrence Radiation Laboratory Report UCRL-18214, May, 1968

9) See for example, R. F. Frosch, R. Hofstadter, J. S. McCarthy, G. K. Noldeke, K. J. van Oostrum, M. R. Yearian, B. C. Clark, R. Herman, and D. G. Ravenhall, Phys. Rev. I74 (1968) 1380

10) W. J. Swiatecki, Proc. Phys. Soc. (London) $\underset{\sim \sim}{A 64}$ (1951) 226 
11) S. Knaak, G. Sussmann, E. Hilf, and H. Buttner, Phys. Letters 23 (1966) 711;

E. Hilf and G. Sussmann, Phys. Letters 21 (1966) 564

12) E. Hilf, Uber Den Oberflachenterm Der Gesamtenergie Der Atomkerme Nach Dem Fermigasmodell, Institut fur Theoretische Physik (Frankfurt) Report (1963)

13) W. J. Swiatecki, The Liquid Drop Theory of Fission, unpublished talk at Gordon Conference, New Hampshire, 26 June 1964

14) B. Vonnegut and R. L. Neubauer, J. Colloid Sci. 7 (1952) 616;

S. A. Ryce and R. R. Wyman, Can. J. Phys. $\underset{\sim 2}{42}$ (1964) 2185;

S. A. Ryce and D. A. Partiarche, Can. J. Phys. 43 (1965) 2192;

S. A. Ryce, Nature 1343 (March 26, 1966)

15) Proceedings of the International Symposium on Electrodynamics at Massachusetts Institude of Technology, March 31-April 1-2, 1969 (International Unions of Theoretical and Applied Mechanics, Pure and Applied Physics)

16) Lord Rayleigh, Phil. Mag. XIV $(1882) 184$

17) C. F. von Weizsäcker, Z. Physik $\underset{\sim}{96}$ (1935) 431

18) J.R. Nix and W. J. Swiatecki, Nuclear Physics 71 (1965) 1; W. J. Swiatecki, Phys. Rev. IOI $_{\text {(1956) 65I; }}$ See also references $19-21,26-27$

19) W. J. Swiatecki, Deformation Energy of a Charged Drop, III, Paper No. $\mathrm{P} / 651$, Proceedings of the Second United Nations International Conference on the Peaceful Uses of Atomic Energy, Geneva, 1958 (United Nations, Geneva, 1958) 
20) J. R. Nix, Further Studies in the Liquid-Drop Theory of Nuclear Fission, Lawrence Radiation Laboratory Report UCRL-17958, JuIy (1968)

21) W. J. Swiatecki, Phys. Rev. 104 (1956) 993

22) J. Jeans: The Mathematical Theory of Electricity and Magnetism (Cambridge University Press, Fifth Edition 1958) pp. 246-248

23) See Horace Lamb, Hydrodynamics (Dover Publications, New York, Sixth Edition, 1945) p. 700, where one is looking at the case of gravitational potential; however the case of electrostatic potential can be easily written down from the results

24) S. A. Ryce and R. R. Wyman, Can. J. Phys. $\underset{\sim 2}{42}$ (1964) 2185

25) See for example J. Jeans: The Mathematical Theory of Electricity and Magnetism (Cambridge University Press, Fifth Edition 1958)

26) s. Cohen and w. J. Swiatecki, Ann of Phys. 19 (1962) 67

27) S. Cohen and W. J. Swiatecki, Ann. of Phys. 22 (1963) 406

28) R. A. Lyttelton, The Stability of Rotating Liquid Masses (Cambridge University Press, 1953)

29) P. Appell, Mecanique Rationnelle, (Gauthier-Villars, Paris, 1932)

30) A. Bohr, B. R. Mottelson, and D. Pines, Phys. Rev. 110 (1958) 936

31) S. G. Nilsson, C. F. Tsang, A. Sobiczewski, Z. Szymański, S. Wycech, C. Gustafson, I-L Lamm, P. Moller, and B. Nilsson, On the Nuclear Structure and Stability of Heavy and Superheavy Nuclei, to be published in Nuclear Physics.

32) See Reference 5;

See also V. M. Strutinski, Nuclear Physics $\underset{\sim \sim}{A 122}$ (1968) I 
33) H. Meldner and P. Roper, private communication to W. D. Myers and W. J. Swiatecki (see Ref. 4);

H. Meldner, Arkiv Fysik 36 (1967) 593, Lawrence Radiation Laboratory Report UCRL-17801 (October 1968);

A. Sobiczewski, F. A. Gareev, and B. N. Kalinkin, Phys. Letters $2 ?$ (1966) 500;

V. M. Strutinski and Yu. A. Muzychka, Proceedings International Confexence of the Physics of Heavy Ions, 13-19 October (1966), Dubna, Vol. 2, p. 51 ;

C. Y. Wong, Phys. Letters 21 (1966) 688;

C. Gustafson, I. I. Lamm, B. Nilsson, and S. G. Nilsson, Arkiv Fysik 36 (1.967) 613;

P. A. Seeger and R. C. Perisho, A Model-based Mass Law and a Table of Binding Energies, Los Alamos Scientific Laboratory Report, IA-3751, UC-34, Physics, TID-4500 (1967)

34) S. G. Nilsson, S. G. Thompson, and C. F. Tsang, Phys. Letters $\underset{\sim}{28 B}$ (1968) 458

35) E. Rost, Phys. Letters $\underset{\sim \sim}{26 \mathrm{~B}}$ (1967) 184

36) R. D. Woods and D. S. Saxon, Phys. Rev. 95 (1954) 577

37) L. C. Gomes, J. D. Walecka, and V. F. Weisskopf, Ann. of Phys 3 (1958) 241

38) R. C. Kennedy, L. Wilets, and E. M. Henley, Phys. Rev. Letters 12 (1964) 35

39) I. G. Moretto, R. C. Gatti, S. G. Thompson, J. R. Huizenga, and J. O. Rasmussen, Phys. Rev. 278 (1969) 1845 
40) W. Stepien and Z. Szymański, Phys. Letters 26은 (1968) 181

41) W. D. Myers and W. J. Swiatecki, Arkiv Fysik 36 (1967) 593

42) Victor F. Weisskopf, Rev. Mod. Phys. 29 (1957) 174

43) R. A. Miller, Some Aspects of the Strutinski Method, Los Alamos Scientific Laboratory Preprint, summer (1967)

44) S. G. Nilsson (Iund Institute of Technology, Sweden) private communication (1969)

45) B. R. Mottelson and S. G. Milsson, Mat. Fys. Skr. Dan. Vid. Selsk $\stackrel{1}{\sim}(1959)$ No. 8

46) D. L. Hendrie, N. K. Glendenning, B. G. Harvey, O. N. Jarvis, H. H. Duhm, J. Saudinos, and J. Mahoney, Phys. Letters 26B (1968) 127;

See also N. K. Glendenning, Proceedings of the International School of Physics, Enrico Fermi, Varenna, Course XL, M. Jean editor (Academic Press, New York, 1968)

47) B. T. Geilikman, Proceedings International Conference on Nuclear Structure, Kingston, 1960, (Toronto University Press, Toronto, 1960) p. 874 ; Also "On The Secondary Shel.zs for Large Nuclear Deformations", I. V. Kurchatov Institute of Atomic Energy preprint (1968)

48) D. A. Arseniev, L. A. Malov, V. V. Pashkevich, and V. G. Soloviev, Dubna preprint E4-3703 (1968)

49) J.R. Nix and G. E. Walker, A Discussion of the Secondary-Minimum Hypothesis For Spontaneously Fissioning Isomers, Los Alamos Scientific Laboratory report, IA-DC-10114 (November 15, 1968) 
50) S. M. Polikanov, V. A. Druin, V. A. Karnaukov, V. L. Mikheev, A. A. Pleve, N. K. Skobolev, V. G. Subotin, G. M. Ter-Akopian, and V.A. Fomichev, Exp. Theor. Phys. 42 (1962) 1464; cf. also S. Bjornholm, J. Borggreen, I. Westgaard, and V. A. Karnaukov, Nuclear Physics $\underset{\sim}{9} 95$ (1967) 513

51) G. N. Flerov and S. M. Polikanov, Compt. Rend. Congr. Int. Phys. Nucl. (Paris 1964) Vol. I, p. 407

52) S. M. Polikanov, Uspeki Fiz. Nauk. 24 (1968) 43

53) N. Iark, G. Sletten, J. Pedersen, and S. Bjфrnholm, Spontaneously Fissioning Isomers in $\mathrm{U}, \mathrm{Np}, \mathrm{Pu}$, and Am Isotopes, Niels Bohr Institute preprint (1969)

54) E. Iynn, The Theory of Neutron Resonance Reactions (Cilaredon Press, oxford. 1968)

55) S. Bjфrnholm, Talk to be given at the Second Symposium on the Physics and Chemistry of Fission (International Atomic Energy Agency), July 28-Aug. 1, 1969, Vienna, Austria; Niels Bohr Institute preprint (1969)

56) V. V. Pashkevich, Conference International Symposium Nuclear Structure, Dubna (1968) 94

57) P. O. Fröman and N. Fröran, J. W. K. B. Approximation, Contribution to the Theory (North-Holland Press, Amsterdarn, 1965)

58) S. G. Nilsson, J. R. Nix, A. Sobiczewski, Z. Szymanski, S. Wycech, C. Gustafson, and P. Moller, Nuclear Physics Alls (1968) 545

59) J.Rayford Nix (Los Alamos Scientific Laboratory) private communication (1969) 
60) J. T. Waber, D. T. Cromer, and D. Liberman, "SCF Dirac-Slater Calculations of the Trans-Lawrencium Elements", Los Alamos Scientific Laboratory Report (to be published)

61) T. C. Tucker, L. D. Roberts, C. W. Nestor, Jr., T. A. Carlson, and F. B. Malik, "Calculation of the Electron Binding Energies and X-Ray Energies for the Superheavy Elements 114, 126, and 140 Using Relativistic SCF Atomic Wave Functions", Oak Ridge National Laboratory Report (to be published)

62) E. M. Burbidge, G. R. Burbidge, W. A. Fowler, and F. Hoyle, Rev. Mod. Phys. $29(1957) 547$

63) S. G. Thompson, R. C. Gatti, L. G. Moretto, H. R. Bowman, and M. C. Michel, paper in preparation.

64) J. J. Wesolowski, W. Johns, R. Jewell, and F. Guy, Phys. Letters $\underset{\sim \sim}{28 B}(1969) 544$

65) G. T. Seaborg; Ann. Rev. Nucl. Sci. 18 (1968) 53-152

66) S. G. Thompson, W. J. Swiatecki, R. C. Gatti, H. R. Bowman, L. G. Moretto, R. C. Jared, and R. M. Latimer, paper in preparation.

67) A. Ghiorso, T. Sikkeland, and M. Nurmia (Lawrence Radiation Laboratory ) private communication

68) G. N. Flerov, Yu. Ts. Oganesyan, Yu. V. Lobanov, V. I. Kuznetsov, V. A. Druin, V.P. Perelygin, K. A. Gavrilov, S. P. Tretiakova, and V. M. Plotko, Atomnaya Energ. 17 (1964) 310; translation: Soviet Atomic Energy 17 (1964) 1046; Phys. Letters 13 (1.964) 73 
Table Captions

Table 1. Summary of topics studied in the thesis.

Table 2. Values of $k$ and $\mu$ employed in the single-particle calculation corresponding to different regions of mass along the betastability line. The first column denotes the mass of the center of each region.

Table 3. Calculated properties of the two-peak spontaneous fiission barriers and shape isomers from potential energy surfaces of. the actinides. The deformations of the ground state and isomeric state are listed. The heights of the two peaks and the excitation energy of the isomeric state are given in MeV above the ground state. It is assumed that the zero-point vibrational energies of the ground state and isomeric state are equal.

Table 4. Experimental properties of shape isomeric states. The first group of columns identifies the nucleus. The second group gives the experimental fission barriers based on the erroneous assumption of a one-peak structure. The excitation energy of the isomeric state is shown in the next group, estimated from threshold measurement and from $(n, f)$ resonance experiments. The next entry gives the ratio of distances between resonances in the isomeric state to those in the ground state. The last column indicates the experimental spontaneous fission half-lives of the isomers. Theoretical values are taken from Table 3 . We are grateful to Dr. S. Bj申rnholm and Dr. J. R. Nix for their help in supplying us with the experimental data. 
Table 5. Beta stability, alpha decay energies and spontaneous fission characteristics of the actinides. In each square classified by $\mathrm{Z}$ and $\mathrm{N}$, the uppermost figure gives the mass excess on ${ }^{12} \mathrm{C}$ scale in $\mathrm{MeV}$. If the nucleus is found to be beta-stable, this number is underlined. The two numbers below give the theoretical and experimental alpha decay $Q_{1}$-values respectively. The integer to the right is the ratio of the experimental spontaneous fission half life to the theoretical value.

Table 6. Table of masses, spontaneous-fission and alpha half-lives for $106<\mathrm{Z}<116$ and $178<\mathrm{N}<189$. The upper number in each square gives the mass excess in ${ }^{12} \mathrm{C}$ scale (see Ref. 41) in MeV. In the line below is listed the spontaneousfission half-life and in parenthesis the barrier height in MeV. The bottom line in each square gives the alpha halflife and the alpha Q-value (in parenthesis). Beta-stable nuclei are underlined.

Table 7. Same as Table 6 , but for the region $116<\mathrm{z}<128$ and $176<\mathrm{N}<190$.

Table 8. Same as Table 6 , but for the region $116<z<128$ and $190<\mathrm{N}<204$.

Table 9. Production of superheavy nuclei by ${ }_{36}^{86}{ }_{\mathrm{Kr}}$.0 beam. The first column identifies the target nucleus. The second column indicates the compound nucleus that is formed by the fusion of the target and the projectile. Assuming that all the excitation energy might be carried away by the emission of four 
neutrons one gets the nucleus shown in the third column. Now assuming that beta decays were extremely slow compared with spontaneous fission and alpha decay one finds the longest lived superheavy nucleus that can be reached as indicated in the fourth column with its major mode of decay. If we let the nucleus in column 4 undergo beta decay one gets the superheavy nucleus shown in the fifth column with its major mode of decay. 
Table 1.

General Introduction

(ㄱ)

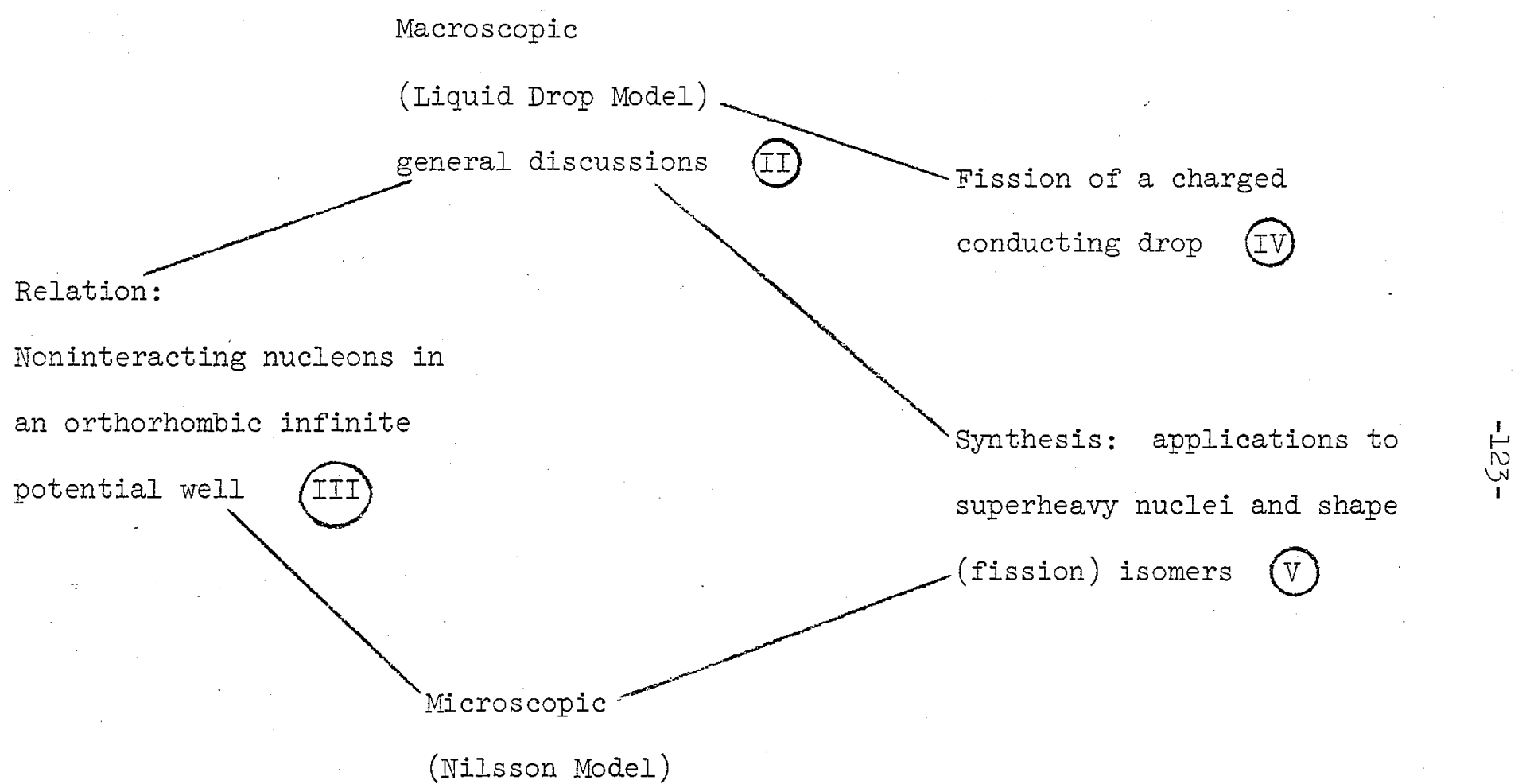

Acknowledgments 
$-124-$

Table 2.

\begin{tabular}{|c|c|c|c|c|}
\hline \multirow[b]{2}{*}{ A value } & \multicolumn{2}{|c|}{ PROTONS } & \multicolumn{2}{|c|}{ NEUTRONS } \\
\hline & $\kappa$ & $\mu$ & $\kappa$ & $\mu$ \\
\hline 165 & 0.0637 & 0.600 & 0.0637 & 0.420 \\
\hline 187 & 0.0620 & 0.614 & 0.0636 & 0.393 \\
\hline 208 & 0.0604 & 0.628 & 0.0636 & 0.367 \\
\hline 225 & 0.0590 & 0.639 & 0.0635 & 0.346 \\
\hline 242 & 0.0577 & 0.650 & 0.0635 & 0.325 \\
\hline 265 & 0.0559 & 0.665 & 0.0635 & 0.296 \\
\hline 285 & 0.0534 & 0.678 & 0.0634 & 0.272 \\
\hline 298 & 0.0534 & 0.686 & 0.0634 & 0.256 \\
\hline 308 & 0.0526 & 0.693 & 0.0633 & 0.244 \\
\hline 320 & 0.0516 & 0.701 & 0.0633 & 0.229 \\
\hline
\end{tabular}


Table 3.

\begin{tabular}{|c|c|c|c|c|c|c|c|c|c|}
\hline \multirow{3}{*}{ Z } & \multirow{3}{*}{$\mathrm{N}$} & \multirow{3}{*}{ A } & \multirow{2}{*}{\multicolumn{2}{|c|}{$\begin{array}{l}\text { Ground State } \\
\text { Deformation }\end{array}$}} & \multirow{3}{*}{$\begin{array}{c}\text { First Peak } \\
\text { Height } \\
\text { above } \mathrm{g} . \mathrm{s} . \\
\mathrm{MeV}\end{array}$} & \multicolumn{3}{|c|}{ Isomeric State } & \multirow{3}{*}{$\begin{array}{c}\text { Second Peak } \\
\text { Height } \\
\text { above g.s. } \\
\text { MeV }\end{array}$} \\
\hline & & & & & & \multicolumn{3}{|c|}{$\begin{array}{ll} & \text { Excitation } \\
\text { Deformation above g.s. }\end{array}$} & \\
\hline & & & $\epsilon$ & $\epsilon_{4}$ & & $\epsilon$ & $\epsilon_{4}$ & $\mathrm{MeV}$ & \\
\hline \multirow[t]{7}{*}{92} & 136 & 228 & 0.15 & -0.06 & 3.4 & \multicolumn{3}{|c|}{ flat region } & $5 \cdot 3$ \\
\hline & 138 & 230 & 0.17 & -0.06 & 3.6 & \multicolumn{3}{|c|}{ flat region } & 6.1 \\
\hline & 140 & 232 & 0.18 & -0.06 & 4.0 & 0.59 & 0.06 & 2.9 & $7 \cdot 5$ \\
\hline & 142 & 234 & 0.195 & -0.05 & 4.9 & 0.585 & 0.05 & 2.5 & 8.1 \\
\hline & 144 & 236 & 0.20 & -0.05 & 5.6 & 0.605 & 0.06 & 2.4 & 8.9 \\
\hline & 146 & 238 & 0.21 & -0.04 & 6.1 & 0.60 & 0.07 & 2.7 & $9 \cdot 5$ \\
\hline & 148 & 240 & 0.23 & -0.03 & 6.8 & 0.64 & 0.06 & 3.2 & 10.0 \\
\hline \multirow[t]{8}{*}{94} & 138 & 232 & 0.18 & -0.06 & 3.5 & 0.65 & 0.08 & 1.9 & 4.2 \\
\hline & 140 & 234 & 0.19 & -0.05 & 4.2 & 0.64 & 0.08 & 2.1 & 5.2 \\
\hline & 142 & 236 & 0.20 & -0.05 & 5.0 & 0.61 & 0.06 & 2.1 & 6.2 \\
\hline & 144 & 238 & 0.215 & -0.04 & 5.8 & 0.60 & 0.06 & 2.2 & 7.0 \\
\hline & 146 & 240 & 0.22 & -0.03 & 6.5 & 0.61 & 0.07 & .2 .5 & 7.6 \\
\hline & 148 & 242 & 0.225 & -0.03 & 69 & 0.61 & 0.07 & 3.0 & 7.95 \\
\hline & 150 & 244 & 0.23 & -0.02 & 72 & 0.62 & 0.07 & 3.6 & 8.3 \\
\hline & 152 & 246 & 0.23 & -0.01 & $7 \cdot 3$ & 0.64 & 0.06 & 3.8 & 8.3 \\
\hline \multirow[t]{6}{*}{36} & 142 & 238 & 0.21 & -0.04 & 5.1 & 0.63 & 0.07 & 1.5 & 4.0 \\
\hline & 144 & 240 & 0.22 & -0.04 & 6.05 & 0.61 & 0.07 & 1.65 & 4.9 \\
\hline & 146 & 242 & 0.225 & -0.03 & 6.7 & 0.60 & 0.07 & 2.2 & 5.5 \\
\hline & 148 & 244 & 0.23 & -0.02 & 7.4 & 0.615 & 0.07 & 2.8 & 6.0 \\
\hline & 150 & 246 & 0.23 & -0.01 & 7.6 & 0.625 & 0.07 & 3.1 & 6.2 \\
\hline & 152 & 248 & 0.23 & -0.01 & 7.7 & 0.65 & 0.07 & 3.5 & 6.4. \\
\hline
\end{tabular}


Table 3. (Continued).

\begin{tabular}{|c|c|c|c|c|c|c|c|c|c|}
\hline \multirow{3}{*}{$\mathrm{Z}$} & \multirow{3}{*}{$\mathbb{N}$} & \multirow{3}{*}{ A } & \multirow{2}{*}{\multicolumn{2}{|c|}{$\begin{array}{l}\text { Ground state } \\
\text { Deformation }\end{array}$}} & \multirow{3}{*}{$\begin{array}{c}\text { First Peak } \\
\text { Height } \\
\text { above g.s. } \\
\text { MeV }\end{array}$} & \multicolumn{3}{|c|}{ Isomeric State } & \multirow{3}{*}{$\begin{array}{l}\text { Second Peak } \\
\text { Height } \\
\text { above g.s. } \\
\text { MeV }\end{array}$} \\
\hline & & & & & & \multicolumn{2}{|c|}{ Deformation } & above g.s. & \\
\hline & & & $\epsilon$ & $\epsilon_{4}$ & & $\epsilon$ & $\epsilon_{4}$ & $\mathrm{MeV}$ & \\
\hline \multirow[t]{5}{*}{$98^{\circ}$} & 146 & 244 & 0.22 & -0.03 & 6.7 & 0.615 & 0.07 & 1.4 & 3.2 \\
\hline & 148 & 246 & 0.23 & -0.02 & 7.45 & 0.625 & 0.07 & 2.0 & 3.75 \\
\hline & 150 & 248 & 0.23 & -0.01 & 7.8 & 0.68 & 0.07 & $2 \cdot 5$ & 4.1 \\
\hline & 152 & 250 & 0.23 & 0.00 & 8.0 & 0.695 & 0.08 & 2.7 & 4.4 \\
\hline & 154 & 252 & 0.235 & 0.01 & $7 \cdot 9$ & 0.705 & 0.08 & 2.6 & 4.0 \\
\hline \multirow[t]{4}{*}{100} & 148 & 248 & 0.23 & -0.01 & $7 \cdot 7$ & 0.73 & 0.08 & 1.2 & 1.8 \\
\hline & 150 & 250 & 0.235 & -0.01 & $7 \cdot 9$ & 0.72 & 0.08 & 1.5 & 2.1 \\
\hline & 152 & 252 & 0.24 & 0.00 & 8.2 & 0.72 & 0.09 & 1.7 & $2 \cdot 3$ \\
\hline & 154 & 254 & 0.24 & 0.01 & 8.1 & 0.73 & 0.09 & 1.6 & 2.1 \\
\hline & 156 & 256 & 0.235 & 0.02 & $7 \cdot 9$ & 0.73 & 0.09 & 1.5 & 1.9 \\
\hline
\end{tabular}


Table 4.

\begin{tabular}{|c|c|c|c|c|c|c|c|c|c|c|c|}
\hline \multicolumn{3}{|c|}{ aveleus } & \multicolumn{3}{|c|}{$\begin{array}{c}\text { Height of Barrier } \\
(\mathrm{MeV})\end{array}$} & \multicolumn{3}{|c|}{$\begin{array}{c}\text { Exc. Energy of sec. Min. } \\
(\mathrm{MeV})\end{array}$} & \multirow{2}{*}{$D_{I I} / D_{I}$} & \multirow{2}{*}{$\begin{array}{c}\text { Fission } \\
\text { Isomer } \\
\text { SF } \\
\tau_{I / 2} \\
(\mathrm{sec})\end{array}$} & \multirow{2}{*}{ Ref. } \\
\hline$Z$ & iN & A & Expt & $\begin{array}{l}\text { The } \\
\text { Ist } P\end{array}$ & $\begin{array}{l}\text { ical } \\
\text { 2nd Peak }\end{array}$ & $D_{I I}^{\text {From }}$ & $\begin{array}{l}\text { From } \\
\text { thresh. } \\
\text { meas. }\end{array}$ & Theory & & & \\
\hline \multirow[t]{2}{*}{92} & 143 & 235 & $5 \cdot 75$ & & & 3 & & & 540 & & a \\
\hline & 144 & 236 & 5.8 & 5.6 & $8.9^{\circ}$ & 2.4 & & 2.4 & 260 & $1.1 \times 10^{-7}$ & b \\
\hline 93 & 145 & 238 & 6.04 & & & & & & & & \\
\hline \multirow[t]{8}{*}{94} & 142 & 236 & & 5.0 & 6.2 & & & 2.1 & & $<2.10^{-9}$ & $c$ \\
\hline & 143 & 237 & & & & & & & & $\sim 10^{-7}$ & c \\
\hline & 144 & 238 & $5 \cdot 3$ & $5 \cdot 8$ & 7.0 & & & 2.2 & & $<2.10^{-9}$ & c \\
\hline & 145 & 239 & 5.5 & & & & & & & $3.10^{-7}$ & c \\
\hline & 146 & 240 & 4.7 & 6.5 & 7.6 & 2.5 & & 2.5 & 100 & $4.10^{-9}$ & d, c \\
\hline & 147 & 241 & 6.3 & & & 2.1 & & & 43 & $3.10^{-8}$ & $e, b$ \\
\hline & 148 & 242 & 5.2 & 6.9 & $7 \cdot 95$ & & & 3.0 & & $5.10^{-8}$ & \\
\hline & 149 & 243 & 5.8 & & & 3.2 & & & 1900 & $6.10^{-8}$ & $a, b$ \\
\hline
\end{tabular}


Table 4 (Continued).

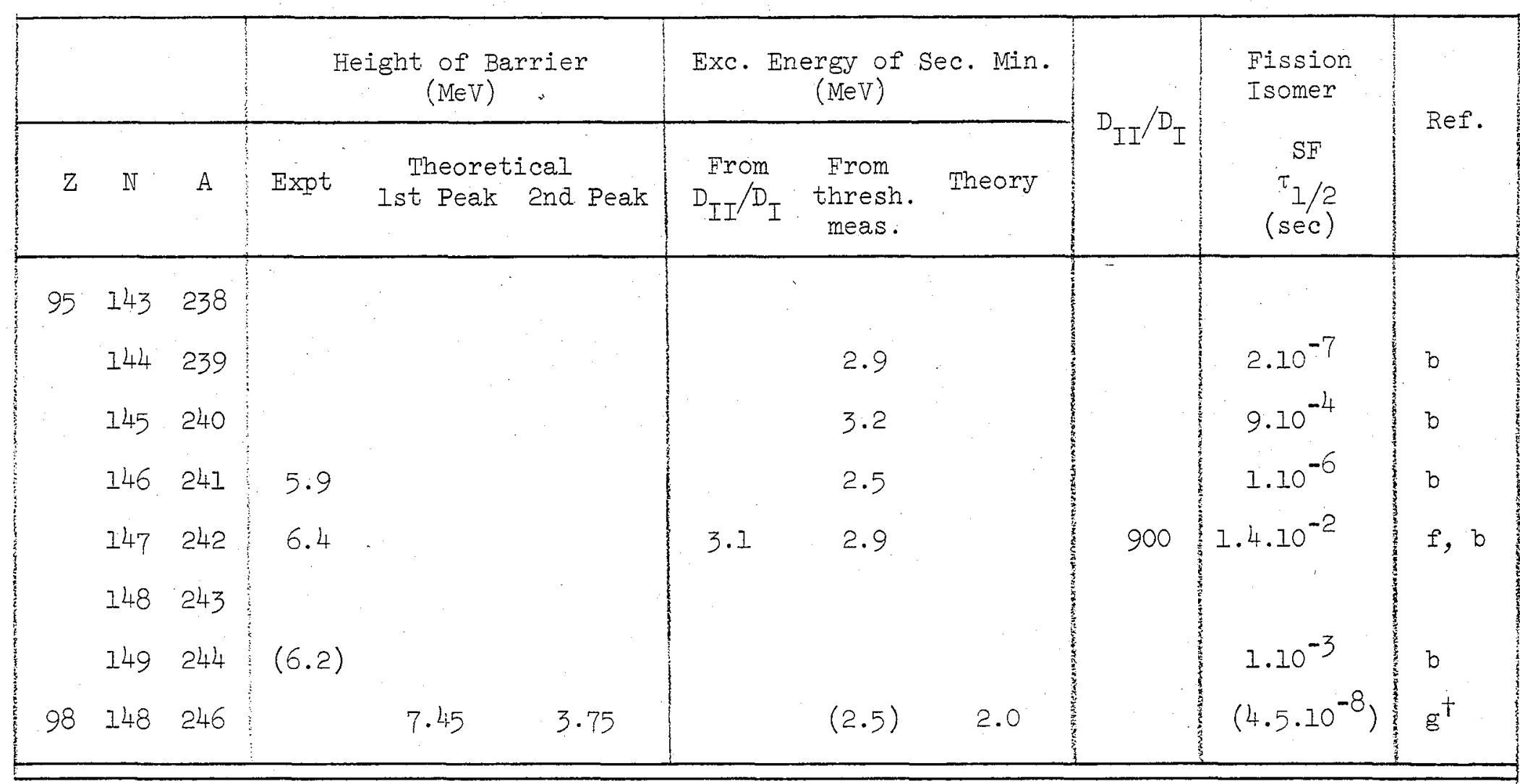

a. Harwell Group

b. Copenhagen Group

c. Seattle Group

d. Saclay Group

e. Euratom Group f. Los Alamos Group

g. Dubna Group

$t$ This result is doubtful. Recent experiments by the Berkeley

Group (Bowman, Cheifetz and Gatti) did not confirm the result.

Expt. Barriers quoted from

Nyers and Swiateckili) 
Table 5 .

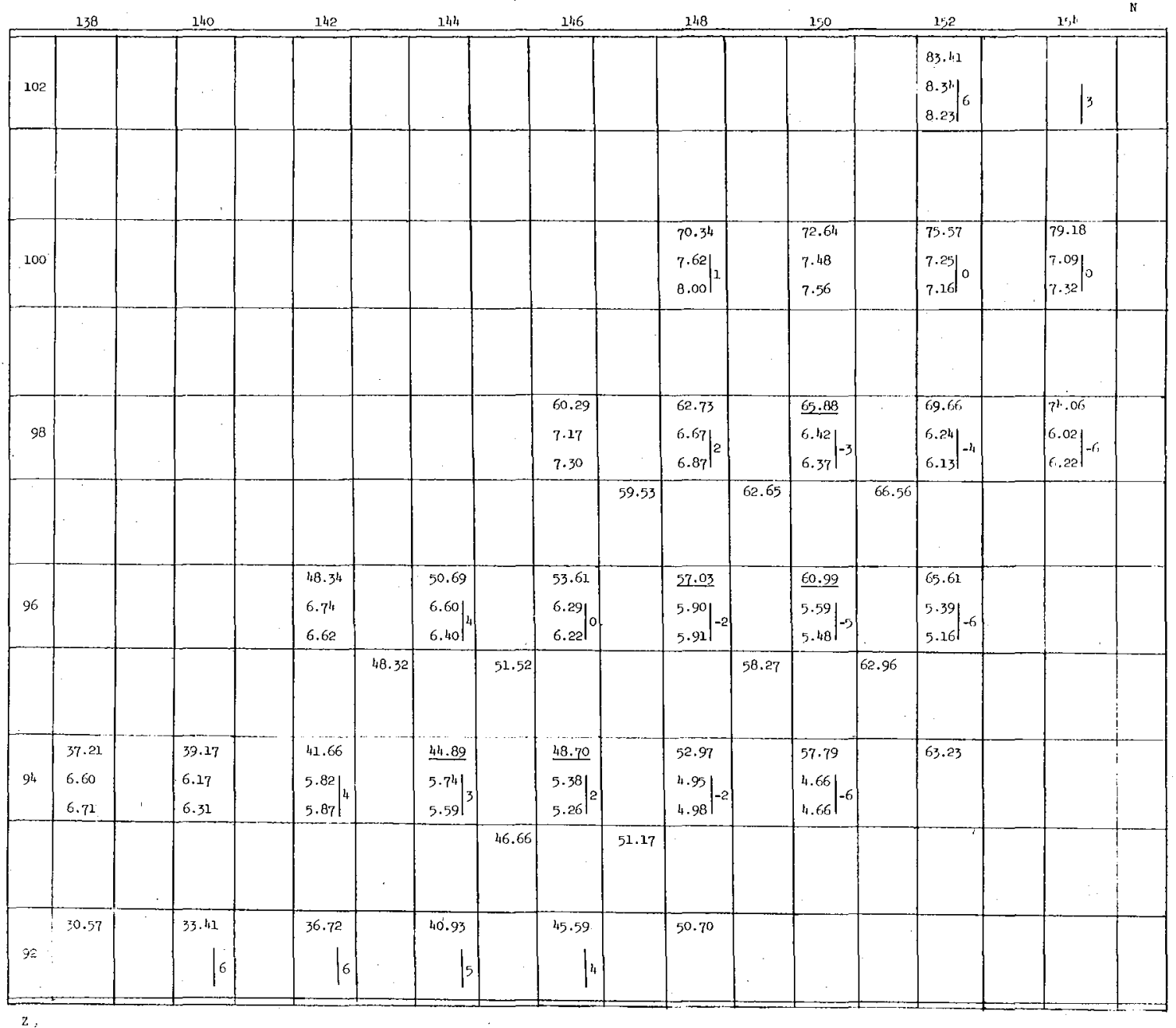


Table 6 .

\begin{tabular}{|c|c|c|c|c|c|c|c|c|c|c|c|c|}
\hline & 178 & 179 & 180 & 181 & 182 & 183 & 184 & 185 & 186 & 287 & 188 & 189 \\
\hline 116 & $\begin{array}{l}187.87 \\
(1 \mathrm{~ms})(5.8) \\
1 \mathrm{~s}(10.14)\end{array}$ & & $\begin{array}{l}190.36 \\
12(7.1) \\
\frac{2}{2} \sin (9.92)\end{array}$ & & $\begin{array}{l}193.14 \\
10^{5} y(8.3) \\
10 s(9.71)\end{array}$ & & $\begin{array}{l}196.42 \\
10^{-1} y(9.4) \\
\frac{1}{2} \min (9.58)\end{array}$ & & \begin{tabular}{|l}
201.30 \\
$10^{121} y(9.4)$ \\
$0.15 \quad(10,17)$
\end{tabular} & & \begin{tabular}{|l|}
206.55 \\
$1 s(10.24)$
\end{tabular} & \\
\hline 125 & 283.02 & & $\begin{array}{l}185.75 \\
10 \min (8.89)\end{array}$ & & $\begin{array}{l}188.85 \\
10 \mathrm{~h}(8.58)\end{array}$ & & $\begin{array}{l}192.45 \\
12(8.45) \\
\end{array}$ & & $\begin{array}{l}197.66 \\
10 s(9.39)\end{array}$ & & \begin{tabular}{|l}
203.25 \\
$10 \min (9.21)$ \\
\end{tabular} & \\
\hline 124 & $\begin{array}{l}178.01 \\
1 \min (5.4)\end{array}$ & 180.09 & $\begin{array}{l}181.00 \\
10^{2} \mathrm{y}(7.0) \\
100(7.97)\end{array}$ & $\begin{array}{l}183.17 \\
1 y(7.71)\end{array}$ & $\begin{array}{l}134.41 \\
10^{9} y(8.3) \\
1 y(7.55)\end{array}$ & $\begin{array}{l}186.56 \\
10^{2} \mathrm{y}(7.20)\end{array}$ & $\begin{array}{l}\frac{188.54}{10^{16 y}(9.6)} \\
10 y(7.40)\end{array}$ & $\begin{array}{l}\frac{191 . \hat{\varepsilon} 9}{1000}(7.87) \\
100\end{array}$ & $\begin{array}{l}\frac{193.88}{10^{15 y}(9.4)} \\
1 \mathrm{da}(8.34)\end{array}$ & $\begin{array}{l}197.32 \\
\sin (8.49)\end{array}$ & $\begin{array}{l}\frac{199.84}{10^{12} \mathrm{y}}(9.4) \\
102(8.09)\end{array}$ & \begin{tabular}{|l}
203.52 \\
$100 \mathrm{~A}(8.00)$
\end{tabular} \\
\hline 13 & 174.43 & & $\begin{array}{l}177.84 \\
10 y(7.35)\end{array}$ & & $\begin{array}{l}181.57 \\
10^{3} y(6.80)\end{array}$ & & $\frac{185.84}{10^{5} y(6.58)}$ & & $\begin{array}{l}191.71 \\
1 y(7.53)\end{array}$ & & $\begin{array}{l}198.00 \\
10 y(7.29) \\
10 y\end{array}$ & \\
\hline 1.22 & $\begin{array}{l}170.60 \\
1 s(4.1)\end{array}$ & 173.03 & $\begin{array}{l}174.45 \\
100(5.7) \\
1 y(7.46)\end{array}$ & $\begin{array}{l}176.93 \\
10^{2} y(7.27)\end{array}$ & $\begin{array}{l}\frac{173.51}{10^{5} y}(6.9) \\
10^{3} y(6.85)\end{array}$ & $\begin{array}{l}180.99 \\
10^{4} y(6.52)\end{array}$ & \begin{tabular}{|l|}
$\frac{183.12}{10^{13} \mathrm{y}}(8.1)$ \\
$10^{4} \mathrm{y}(6.54)$
\end{tabular} & $\begin{array}{l}186.40 \\
10^{2} y(7.20)\end{array}$ & $\begin{array}{l}189.32 \\
10^{3-3} y(8.1) \\
1 y(7.50)\end{array}$ & $\begin{array}{l}193.09 \\
1000(7.65)\end{array}$ & $\begin{array}{l}195.94 \\
10^{12} y(8.1) \\
10 y(7.24)\end{array}$ & $\begin{array}{l}199.95 \\
10 y(7.26)\end{array}$ \\
\hline$x$ & 168.08 & & $\begin{array}{l}172.34 \\
10 y(7.05) \\
\end{array}$ & & $\begin{array}{l}\frac{176.83}{12} \\
10^{5} y(6.38)\end{array}$ & \begin{tabular}{|l|}
179.47 \\
\end{tabular} & $\begin{array}{l}181.75 \\
10^{7} \mathrm{y}(6.03) \\
\end{array}$ & & $\begin{array}{l}288.28 \\
10^{2} \mathrm{y}(6.98)\end{array}$ & & $\begin{array}{l}195.23 \\
10^{3} y(6.72)\end{array}$ & \\
\hline 210 & $\begin{array}{l}164.54 \\
(1 \mathrm{~ms})(3.2) \\
10 y(7.20)\end{array}$ & 167.33 & $\begin{array}{l}\frac{169.25}{10 \min ^{2}}(4.3) \\
10^{2} y(6.85)\end{array}$ & 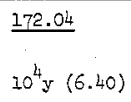 & $\begin{array}{l}\frac{174.1 k}{10^{4} y}(5.5) \\
10^{6} y(5.14)\end{array}$ & \begin{tabular}{|l|}
176.87 \\
$20^{9} \mathrm{y}(5.63)$
\end{tabular} & \begin{tabular}{|l}
$\frac{179.39}{10^{10}}$ \\
$10^{8} y(6.8)$ \\
$15.76)$
\end{tabular} & $\begin{array}{l}183.01 \\
10^{5} y(6.24)\end{array}$ & $\begin{array}{l}186.27 \\
10^{10} \mathrm{y}(5.7) \\
10^{2} y(6.73)\end{array}$ & $\begin{array}{l}190.36 \\
10^{2} y(6.86)\end{array}$ & \begin{tabular}{|l}
193.54 \\
$10^{9}$ \\
$10^{4} y(6.8)$ \\
$16.45)$
\end{tabular} & $\begin{array}{l}197.88 \\
10^{4} y(6.35)\end{array}$ \\
\hline 109 & 262.86 & & 168.02 & 171.10 & 173.29 & 175.18 & $\begin{array}{l}173.87 \\
10^{11} y(5.24)\end{array}$ & 132.66 & $\begin{array}{l}186.08 \\
10^{5} y(6.21) \\
\end{array}$ & & 193.68 & \\
\hline 108 & $\begin{array}{l}159.97 \\
10^{3} y(6.38)\end{array}$ & 263.21 & $\begin{array}{l}165.57 \\
108(3.2) \\
10^{4} y(6.23)\end{array}$ & 168.32 & $\begin{array}{l}271.20 \\
10^{2} y(4.3) \\
10^{8} y(5.57)\end{array}$ & 174.34 & $\begin{array}{l}177.21 \\
10^{8}(5.8) \\
10^{13} y(4.89)\end{array}$ & $\begin{array}{l}181.07 \\
109.5(5.59)\end{array}$ & $\begin{array}{l}184.66 \\
10^{8} y(5.9) \\
10^{5} y(5.86)\end{array}$ & 189.10 & $\begin{array}{l}192.60 \\
10^{7} \mathrm{y}(5.8)\end{array}$ & \\
\hline 107 & & & & & 171.20 & 174.49 & 177.44 & & & & & \\
\hline 106 & & & & & $\begin{array}{l}169.79 \\
100 .(3.9) \\
10^{12} \mathrm{y}(4.97)\end{array}$ & 173.25 & \begin{tabular}{|l}
176.37 \\
$10^{7} y(5.3)$
\end{tabular} & & & & & \\
\hline
\end{tabular}


Table 7.

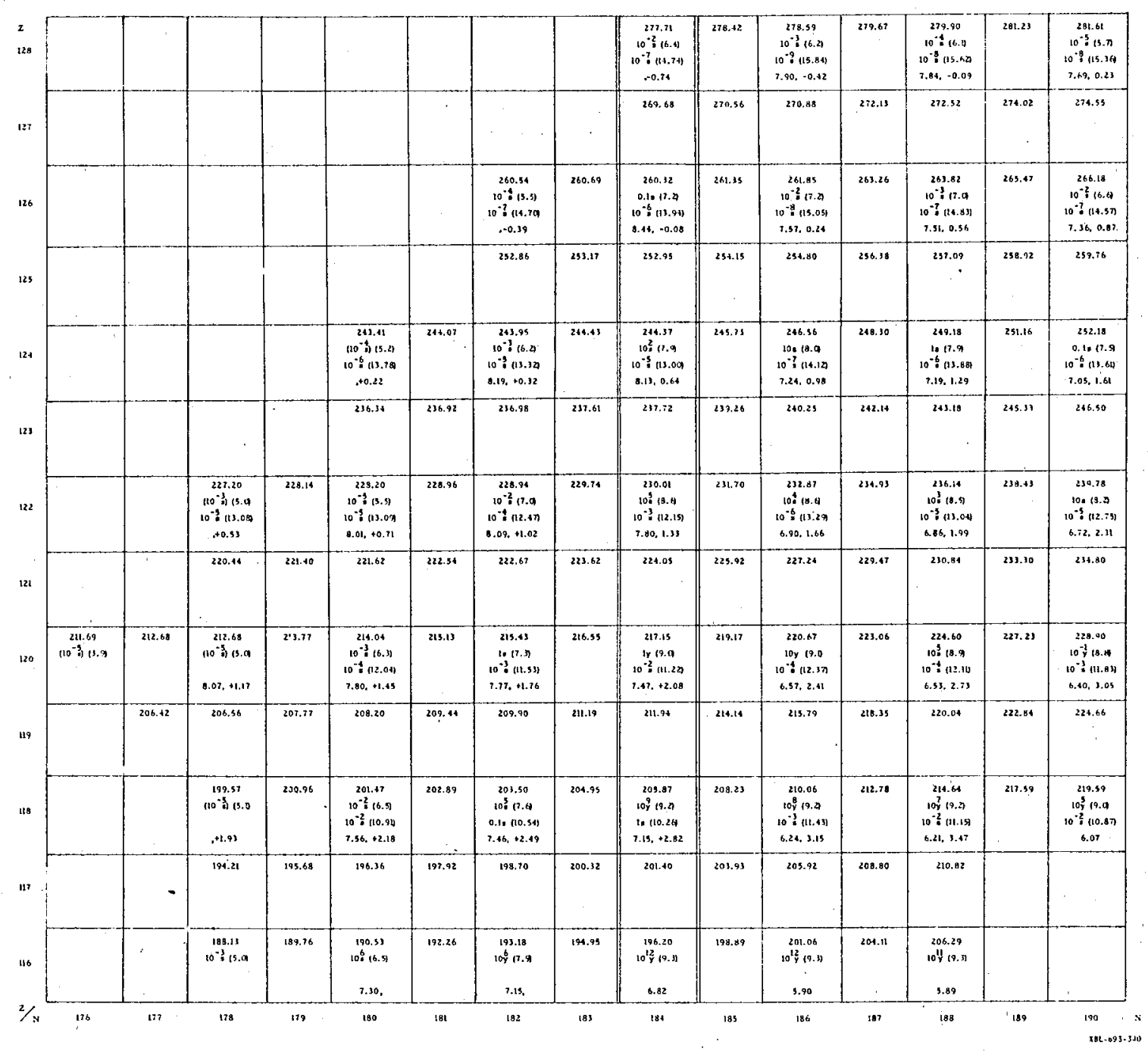


Table 8.

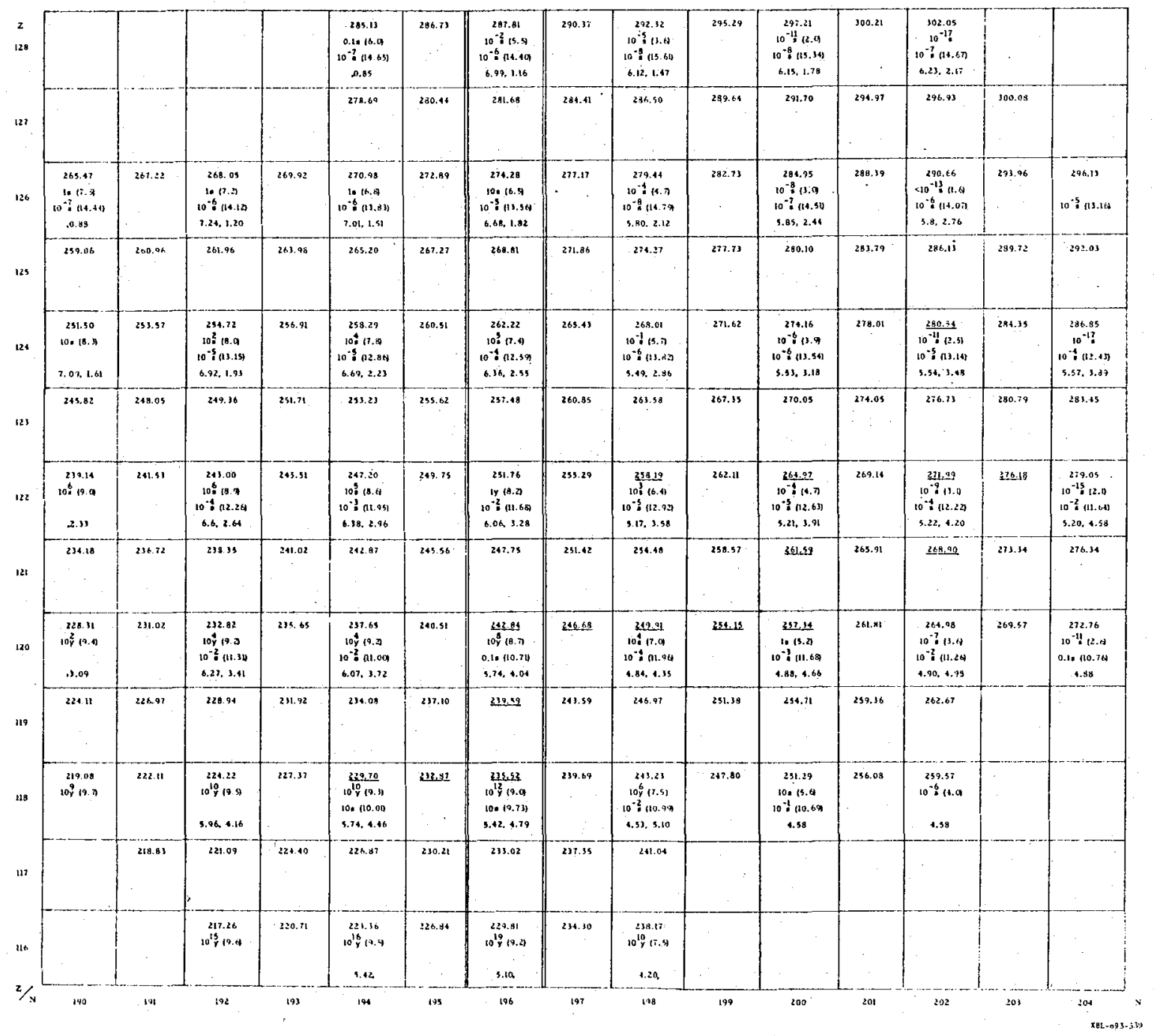


Table 9.

Production of Superheavy Nuclei by ${ }^{86} 6^{\mathrm{Kr}} 50$. Projectile

\begin{tabular}{|c|c|c|c|c|c|c|c|c|c|c|c|c|c|}
\hline \multicolumn{4}{|c|}{ Target } & \multicolumn{2}{|c|}{$\begin{array}{l}\text { Compound } \\
\text { Nucleus }\end{array}$} & \multicolumn{2}{|c|}{$\begin{array}{c}\text { After } \\
\text { emitting } \\
4 n\end{array}$} & \multicolumn{3}{|c|}{$\begin{array}{c}\text { Longest-lived } \\
\text { nuclei reached } \\
\text { after competition } \\
\text { between s.f. and } \\
\text { successive } \\
\alpha \text {-decay }\end{array}$} & \multicolumn{3}{|c|}{ After $\beta$-decay } \\
\hline & $A$ & $z$ & N & Z & $\mathbb{N}$ & $\mathrm{Z}$ & $N$ & Z & $\mathbb{N}$ & $\begin{array}{l}\text { Major } \\
\text { Decay }\end{array}$ & $\mathrm{Z}$ & N & $\begin{array}{l}\text { Major } \\
\text { Decay }\end{array}$ \\
\hline $\mathrm{Pb}$ & 208 & 82 & 126 & 118 & 176 & 118 & 172 & \multicolumn{3}{|c|}{$(s . f)$} & & & \\
\hline Po & 210 & 84 & 126 & 120 & 176 & 120 & 172 & \multicolumn{3}{|c|}{$(s . f)$} & & & \\
\hline $\mathrm{Rn}$ & & & & & & & & & & & & & \\
\hline $\mathrm{Ra}$ & 226 & 88 & 138 & 124 & 188 & 124 & 184 & 118 & 178 & $\alpha\left(10^{-3} \mathrm{~s}\right)$ & 112 & 184 & $\alpha\left(10^{4} \mathrm{y}\right)$ \\
\hline Th & 232 & 90 & 142 & 126 & 192 & 126 & 188 & 116 & 178 & $\alpha\left(10^{-1} s\right)$ & 112 & 182 & $\alpha\left(10^{2} y\right)$ \\
\hline $\mathrm{U}$ & 238 & 92 & 146 & 128 & 196 & 128 & 192 & 114 & 178 & $\alpha\left(10^{3} \mathrm{~s}\right)$ & 110 & 182 & $\alpha\left(10^{2} y\right)$ \\
\hline Pu & 244 & 94 & 150 & 130 & 200 & 130 & 196 & 114 & 180 & $\alpha\left(10^{4} s\right)$ & 112 & 182 & $\alpha\left(10^{2} y\right)$ \\
\hline $\mathrm{Cm}$ & 248 & 96 & 152 & 132 & 202 & 132 & 198 & 114 & 180 & $\alpha\left(10^{4} s\right)$ & 112 & 182 & $\alpha\left(10^{2} y\right)$ \\
\hline
\end{tabular}




\section{Figure Captions}

Fig. 1. Single particle energies for a Hill-Wheeler box as a function of the deformation co-ordinate $\alpha$. The other deformation co-ordinate $\gamma$ has been set to zero. A large gap in the energy levels is indicated by a number which gives the number of levels below the gap.

Fig. 2. The configuration in momentum space for the Hill-Wheeler box. only the $\left(\mathrm{k}_{\mathrm{x}}, \mathrm{k}_{\mathrm{y}}\right)$ plane is shown. The positions of the dot:s in units of $k_{x}=\pi / a=2 w_{1}, k_{y}=\pi / b=2 w_{2}$, and $\mathrm{k}_{z}=\pi / c=2 \mathrm{w}_{3}$ give the quantumnumbers of the levels. The fermi momentum $k_{F}$ is the momentum of the highest level that is filled. The effective Fermi momentum $\mathrm{g}$ is defined such that the volumes of the bumps and dips cancel.

Fig. 3. A bump and a dip on the effective Fermi surface after averaging with respect to orientation.

Fig. 4a. The energy of particles in a cubic Hill-Wheeler box as a function of particle number calculated in four different ways: (1) Using the approximate expression $E^{\prime}$ with only the N-term, $\mathrm{E}^{\prime}(\mathrm{N}) ;(2) \mathrm{E}^{\prime}$ with the $\mathrm{N}$-term and the $\mathrm{N}^{2 / 3}$-term, $\mathrm{E}^{\prime}\left(\mathrm{N}^{2 / 3}\right)$; (3) $E^{\prime}$ up to the $N^{1 / 3}$ term, $E^{\prime}\left(N^{1 / 3}\right)$; (4) The exact calculation $\mathrm{E}$. The volume of the box is assumed proportional to N. The unit of the ordinate is in $\pi^{2} h^{2} / 2 M \beta^{2}$ where $\beta$ is given by $V=\beta^{3} \mathrm{~N}$.

-D. Name as Fig. $4 a$ for an oblate Hill-Wheeler box. 
Fig. 4c. Same as Fig. 4a for a prolate Hill-Wheeler box.

4d. Same as Fig. 4a for a Hill-Wheeler box with three unequal sides.

Fig. 5a. The enexgy of $N=60$ particles in a Hill-Wheeler box as a function of the deformation parameter $\alpha(\gamma=0)$ calculated in three different ways: (I) Using the approximate expression $E^{\prime} \quad$ up to $N^{2 / 3}$-term, $E^{\prime}\left(N^{2 / 3}\right) ;(2) E^{\prime}$ up to $N^{1 / 3}$ term, $E^{\prime}\left(N^{1 / 3}\right)$; (3) The exact calculation, E. The results for $E^{\prime}$ wi.th only the N-term is independent of $\alpha$ and is not shown. The ordinate has a different unit from that of Figs. 4. It is converted to the latter by multiplying by $\mathbb{N}^{-2 / 3}$.

5b. Same as Fig. 5a for the case of $N=68$.

Fig. 6a. The energy differences between $E$ and $E^{\prime}\left(\mathbb{N}^{2 / 3}\right)$ and between $\mathrm{E}$ and $\mathrm{E}^{\prime}\left(\mathrm{N}^{1 / 3}\right)$ as a function of the particle number $\mathbb{N}$ for a cubic Hill-Wheeler box. See Figures 4.

6b. Same as Fig. 6a for an oblate Hill-Wheeler box.

6c. Same as Fig. 6a for a prolate Hill-Wheeler box.

6d. Same as Fig. 6a for a Hill-wheeler box with three unequal sides.

Fig. 7a. The energy differences between $E^{2}$ and $E^{\prime}\left(\mathbb{N}^{3 / 3}\right)$ and between $\mathrm{E}$ and $\mathrm{E}^{\prime}\left(\mathrm{N}^{2 / 3}\right)$ as a function of the deformation parameter $\alpha, \quad(r=0)$ for the case of $N=60$ particles in a HillWheeler box. See Figures 5 . 7b. Same as Fig. Ta for the case of $N=68$. 
Fig. 8. Energy excess $\xi$ over a spherical drop as a function of deformation.

Fig. 9. Energy excess $\xi$ over a spherical drop as a function of deformation for different values of the fissility parameter $x$.

Fig. 10. The energy change in the division of a volume-charged drop into $n$ ecual parts, as a function of the fissility parameter $x$. The ordinate is just $\xi_{\mathrm{R}}$. Taken from Ref. 19 .

Fig. 11. The maximum and minimum radii of saddle point shapes of a volume-charged drop as a function of the fissility parameter $\mathrm{x}$. The results for the symmetrical saddle point shapes are given by the solid curves, and the results for the asymmetric saddle point shapes by the dashed curve. Adapted from Ref. 20.

Fig. 12. The energy change in the division of a volume-charged drop into two spheres as a function of the fractional volume of one of the spheres for various values of $x$. Taken from Ref. 24 .

Fig. 13. Same as Fig. 12 for the case of a conducting drop.

Fig. 14. Shapes in the symmetry $N=2$ family of equipotential surfaces.

Fig. 15. Shapes in the symmetry $\mathbb{N}=3$ family of equipotential surfaces.

Fig. 16. The maximum and minimum radii of the symmetric saddle point shapes of a conducting drop as a function of the fissility parameter $x$. Different curves correspond to the restriction to different families of shapes indicated by the values of $\mathbb{N}$.

Fig. 17. Saddle point shapes within the symmetric $N=6$ family for various values of $x$. The RMS values are also indicated. 
Fig. 18. The energy excess $\xi$ (in units of $E_{S}{ }^{(0)}$ ) over a sphere of the symmetric saddle point shapes as a function of the fissility parameter $x$ is shown in solid curves. It is calculated on the $\mathbb{N}=6$ parameterization. The broken curve indicates the results for a volume-charged drop taken from Ref. 27.

Fig. 19. Shapes described by the plane of the deformation parameters $\epsilon$ and $\epsilon_{4}$. A sphere corresponds to $\epsilon=0$ and $\epsilon_{4}=0$. Spheroids have their $\epsilon_{4}=0$.

Fig. 20. Relation between deformation co-ordinates $\epsilon, \epsilon_{4}$, and $\alpha_{2}$, $\alpha_{4}$. Note that the spheroid contains some $\alpha_{4}$ (as well as $\alpha_{6}$ etc. not shown in the figure).

Fig. 21. Single-proton level diagram for spherical potential. Parameters are fitted ${ }^{3)}$ to reproduce observed deformed single-particle level order at $A \approx 165$ and 242 , and are extrapolated linearly to the other regions. E. Rost's preaicted level order 35 ) for $A=298$ is exhibited for comparison

Fig. 22. Analogous to Fig. 21, valid for neutrons.

Fig. 23. Single-proton levels $A \approx 298 ; \kappa=0.0534 ; \mu=0.686$, $\epsilon_{4}=0$.

Fig. 24. Effect of various terms in total energy as a function of deformation. Long-dashed curve marks simple sum of single-particle energies, for dotted curve Coulomb energy is added, for dotdashed curve also pairing $(G \propto S)$ is included, for shortdashed curve the Strutinsky normalisation is applied. In all these cases it is assumed that. $\epsilon_{4}=0$. In the last case 
(solid curve) also the effect of the $\epsilon_{4}$-degree of freedom is included.

Fig. 25. Sum of single-particle, pairing and Coulomb energies without

Strutinski normalization as function of $\epsilon, \epsilon_{4}$. At large distortions the energy ultimately rises beyond $+15 \mathrm{MeV}$ (limit for the plot).

Fig. 26. A sketch of the exrors $\left|\xi_{S}\right|$ and $\left|\xi_{I}\right|$ in the Strutinski. Prescription as a function of the smearing width $r$, for various order $m$ of the correction factor $\mathrm{F}_{\mathrm{m}}$.

Fig. 27. Shell corrections evaluated by the strutinski method as a function of the shell-smearing parameter $\gamma$ for case of neutrons of ${ }^{242} \mathrm{Pu}$. Energies corresponding to three different distortions are considered.

Fig. 28. Same as Fig. 27, but for neutrons of ${ }^{208} \mathrm{~Pb}$.

Fig. 29. Experimental and theoretical mass values for $150<A<340$ plotted relative to the spherical liquid drop value as of Ref. 4I.

Fig. 30. Theoretical deformations, $\left(\epsilon, \epsilon_{4}\right)$, of ground state nuclei in the rare earth region.

Fig. 31. Theoretical deformations, $\left(\epsilon, \epsilon_{4}\right)$, of ground state nuclei in the actinide region.

Fig. 32. Empirical rare earth $\beta_{4}$-values (filled circles) obtained through the analysis of Ref. 46 compared to the present calculations before the inclusion of the Strutinski normalisation. The effect of the latter is less than 0.01 in magnitude. 
Fig. 33a. Total energy minimized.w.r.t. $\epsilon_{4}$ for each $\epsilon$ as function of $\epsilon$ for isotopes of $92^{U}$. Dashed curve corresponds to $G$ set constant while the solid line is based on assumption that $G$ is proportional to the nuclear surface area.

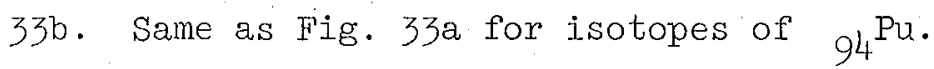

33c. Same as Fig. 33 a for isotopes of $96^{\mathrm{cm}}$.

33a. Same as Fig. $33 a$ for isotopes of $98^{\mathrm{Cf}}$.

33e. Same as Fig. 33a for isotopes of $100 \mathrm{Fm}$. The extra dotdashed curve added for ${ }^{256} \mathrm{Fm}$ represents the new total energy for the case' $G \propto S$ when the nuclear potential parameters are modified from those relevant for $A=242$ to those for $A=265$. As can be seen the barrier change is very small.

33f. Same as Fig. 33 a for isotopes of $102^{\text {No }}$.

33g. Same as Fig. 33 a for isotopes of $Z=104$.

33h. Same as Fig. 33a for isotopes of $\mathrm{Z}=106$.

33i. Same as Fig. 33a for isotopes of $Z=108$.

33j. Same as Fig. 33a for isotopes of $Z=110$.

33k. Same as Fig. 33a for isotopes of $z=1.12$.

336. Same as Fig. 33a for isotopes of $z=114$.

Fig. 34. Potential energy minimized with respect to $\epsilon_{4}$ as a function of $\epsilon$ for various nuclei to illustrate the effect of shell structure of a liquid drop background. The broken curves correspond to liguid drop fission barriers. The solid curves are the barrier after inclusion of shell and pairing effects. 
Fig. 35. The two-peak barrier as a function of mass number for $Z=92-100$.

Fig. 36a. Same as Fig. 33a for isotopes of $82^{\mathrm{Pb}}$.

36b. Same as Fig. 33a for isotopes of $84^{\text {Po. }}$

36c. Same as Fig. $33 a$ for isotopes of $86^{\mathrm{Rn}}$.

36a. Same as Fig. 33a for isotopes of $88^{\text {Ra. }}$

36e. Same as Fig. 33a for isotopes of $90^{\text {Th }}$.

F'ig. 37a. Total-energy surface in $\left(\epsilon, \epsilon_{4}\right)$ plane for ${ }^{25}{ }^{2} \mathrm{Fm}$ after application of the strutinski normalisation. This figure corresponds to a somewhat earlier calculation and employs $G=$ const and a different pairing cut-off than described in the present paper. More recent calculations are exhibited in Fig. 33.

37b. Same as Fig. 37 a valid for 290114.

Fig. 38a. Liquid-drop energy surface for ${ }^{252} \mathrm{Fm}$.

38b. Liquid-drop energy surface for ${ }^{290} 114$.

Fig. 39a. Shell and pairing energy contributions for ${ }^{252} \mathrm{Fm}$. For further details see Fig. 37 a.

39b. Same as Fig. 39a for $290_{114 .}$

Fig. 40. Spontaneous fission half lives of $z=114$ and 110 isotopes as functions of the inertial parameter $B$ for barrier penetration. Three estimates of $B$ are given. For further explanations, see text.

Fis. 41. Contours of theoretical half-lives for $106<\mathrm{z}<128$ and $170<\mathrm{N}<204$. The thick dark lines are contours of 
spontaneous fission half-lives. The broken lines are contours of alpha half-lives. Beta stable nuclei are shaded.

Fig. 42. Periodic Table exhibiting predicted locations of new elements. 


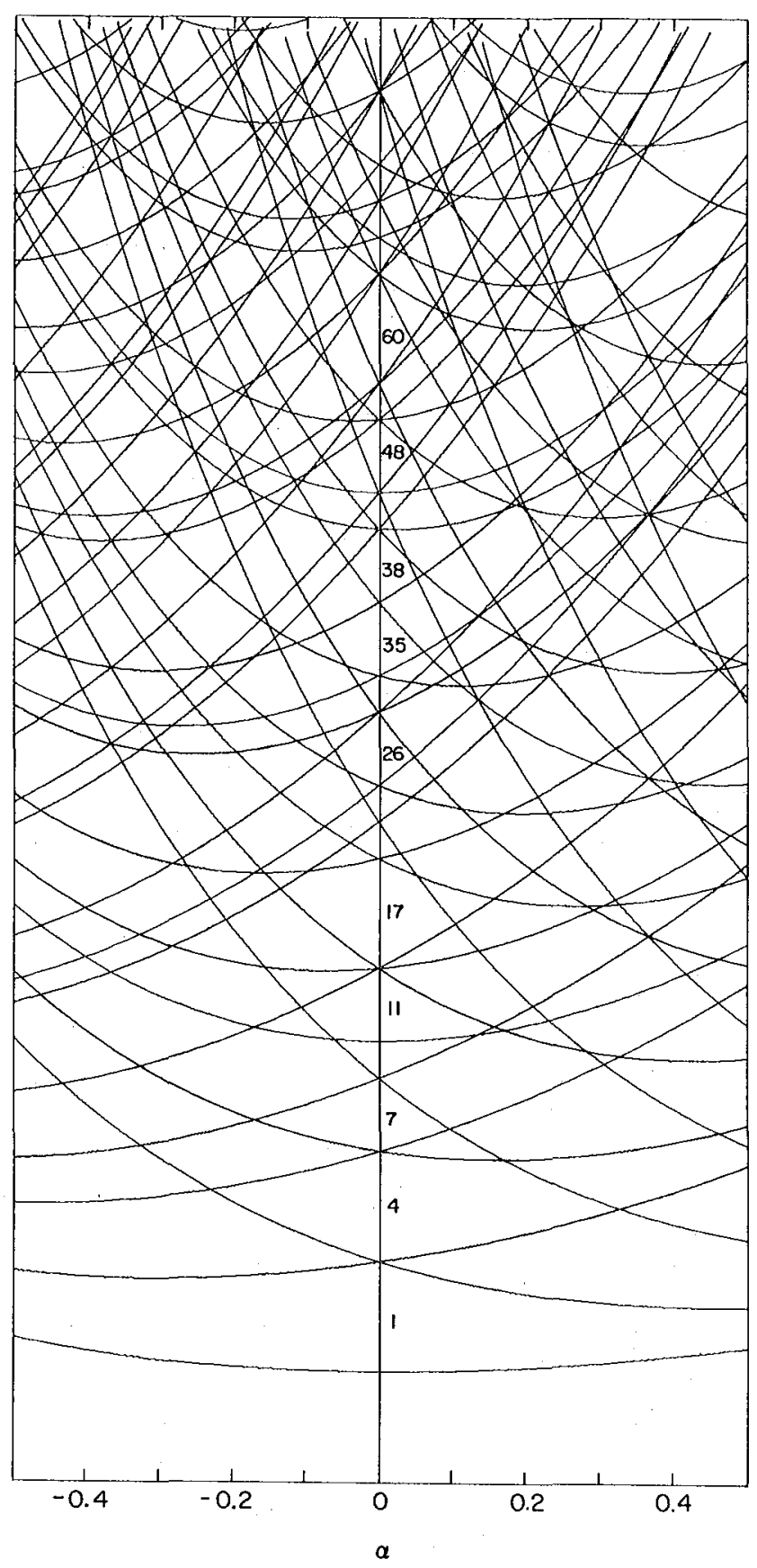

XBL693-2263

Fig. I 


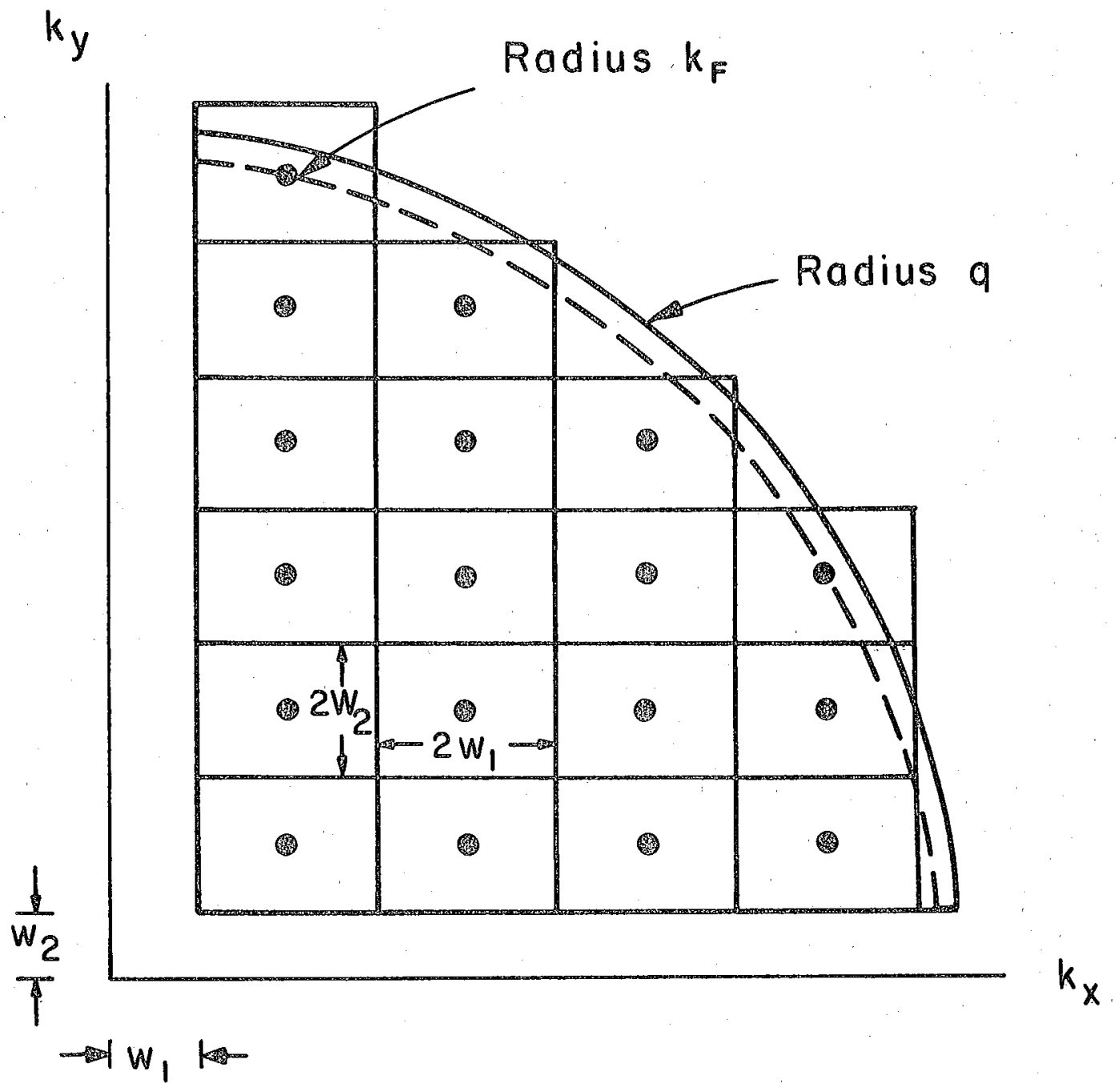

$X B L 694-2517$

Fig. 2 
$-144-$

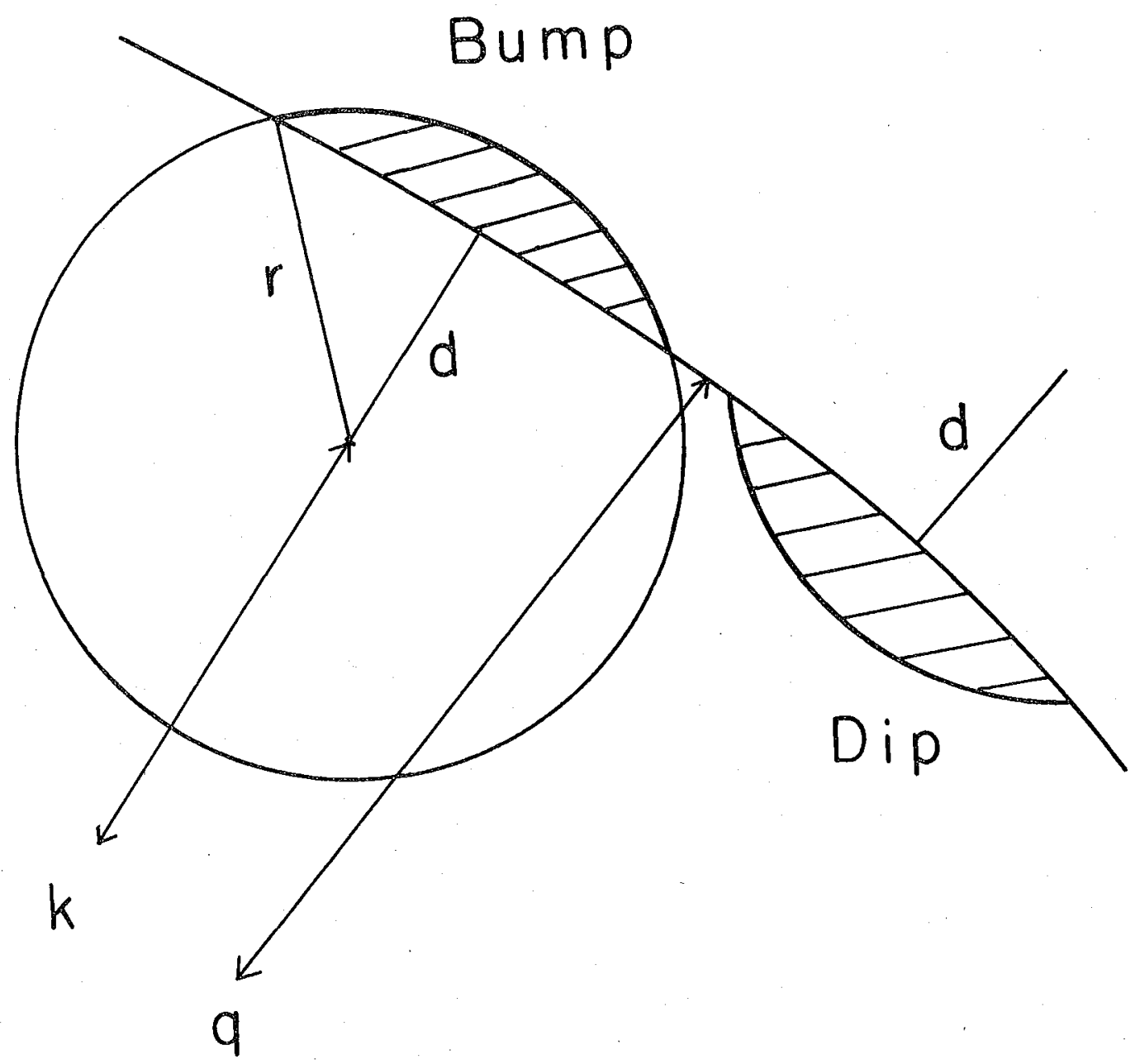

XBL 695-580

Fig. 3 


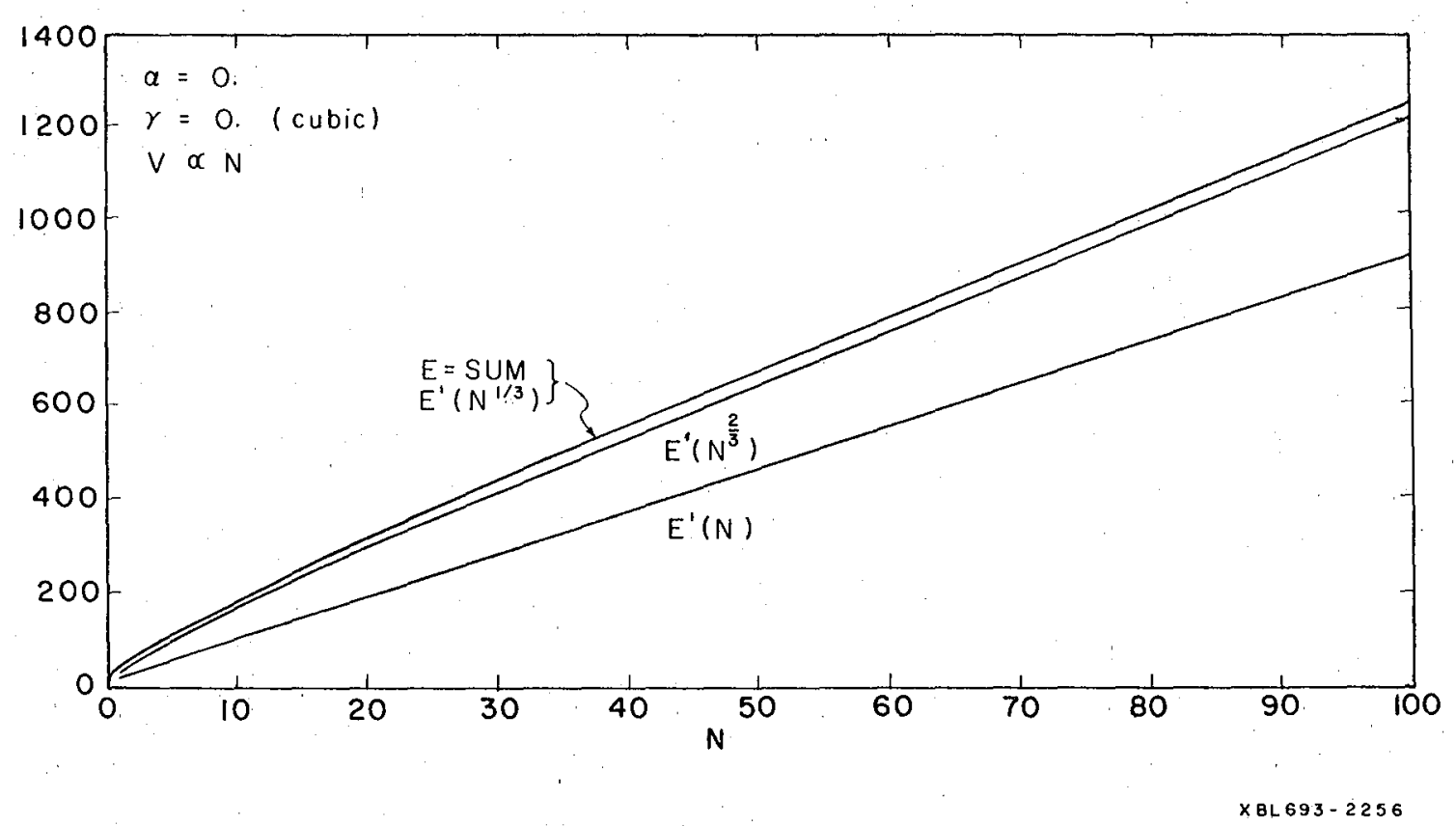

Fig. $4 a$ 
$-146-$

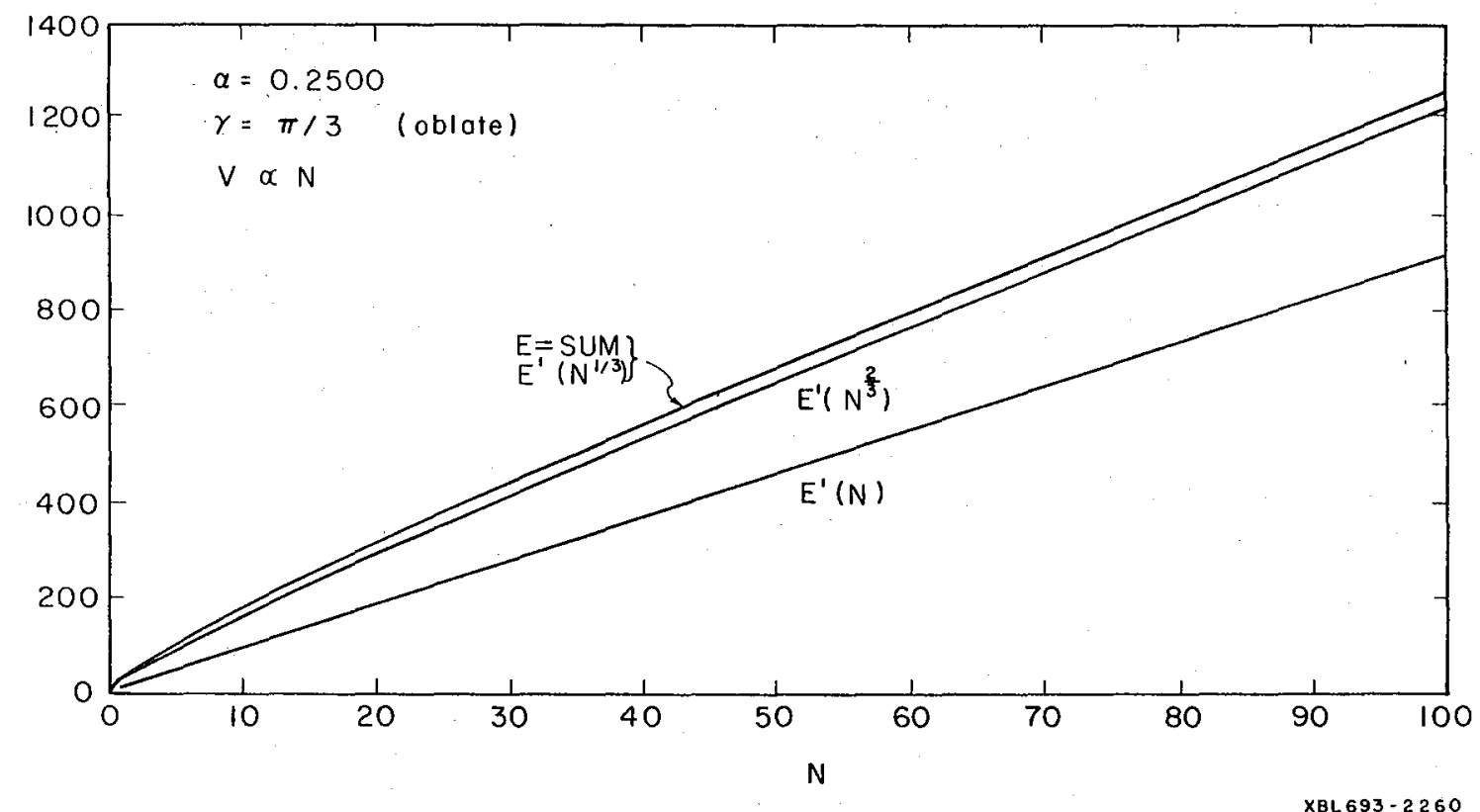

Fig. 4b 


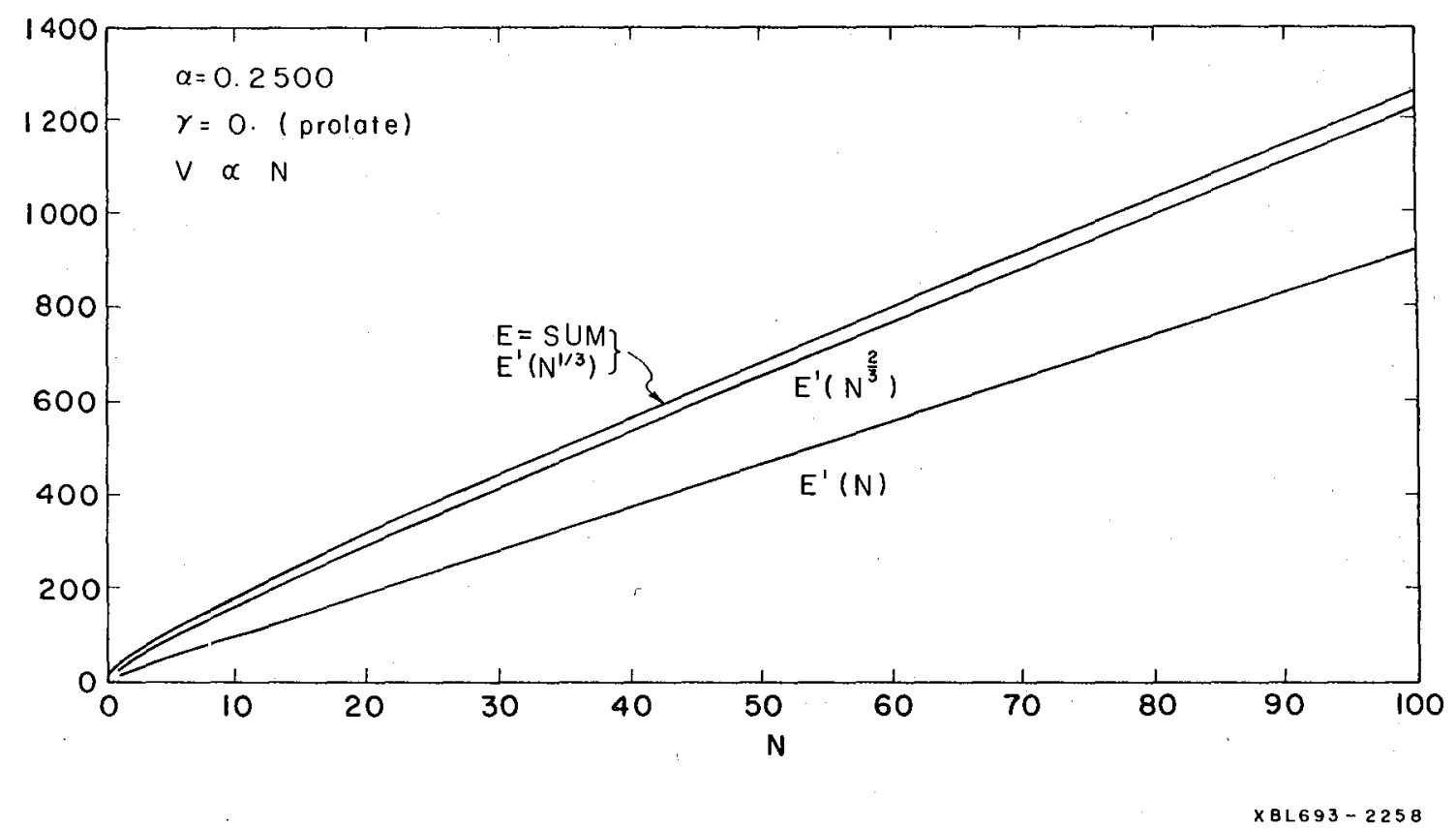

Fig. 4c 
$-148-$

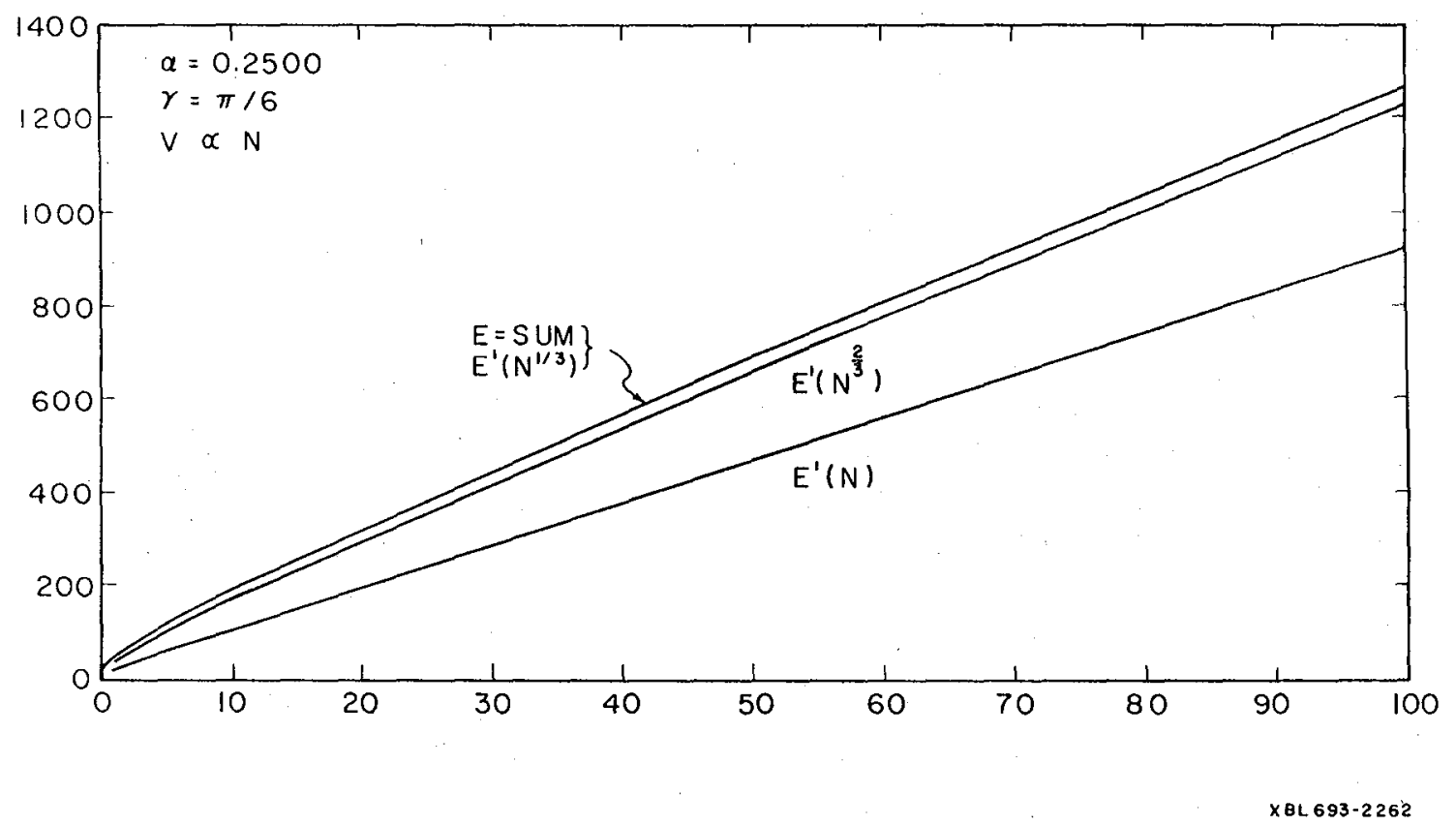

Fig. 4d 
$-149-$

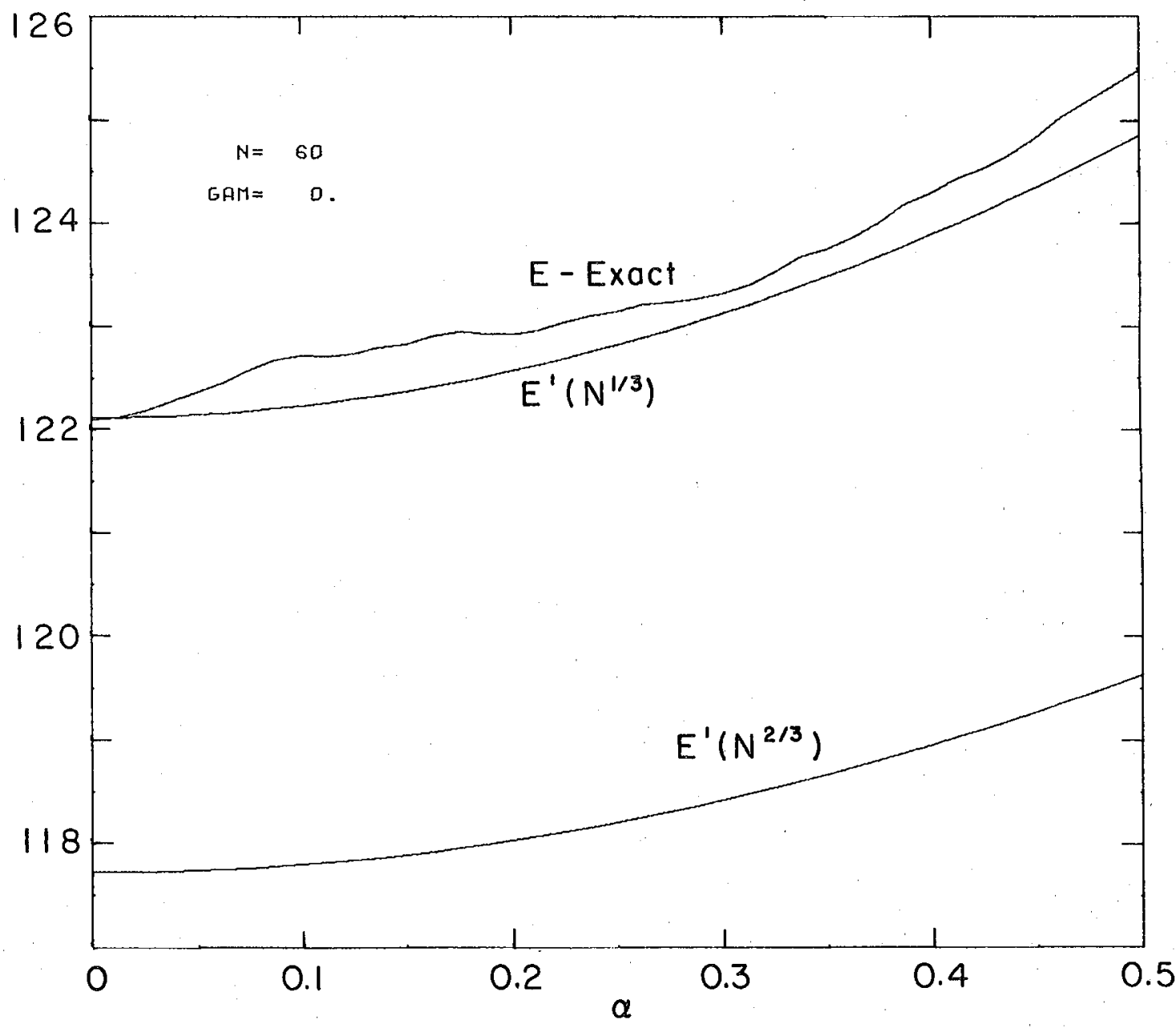

XBL693-2276

Fig. $5 a$ 


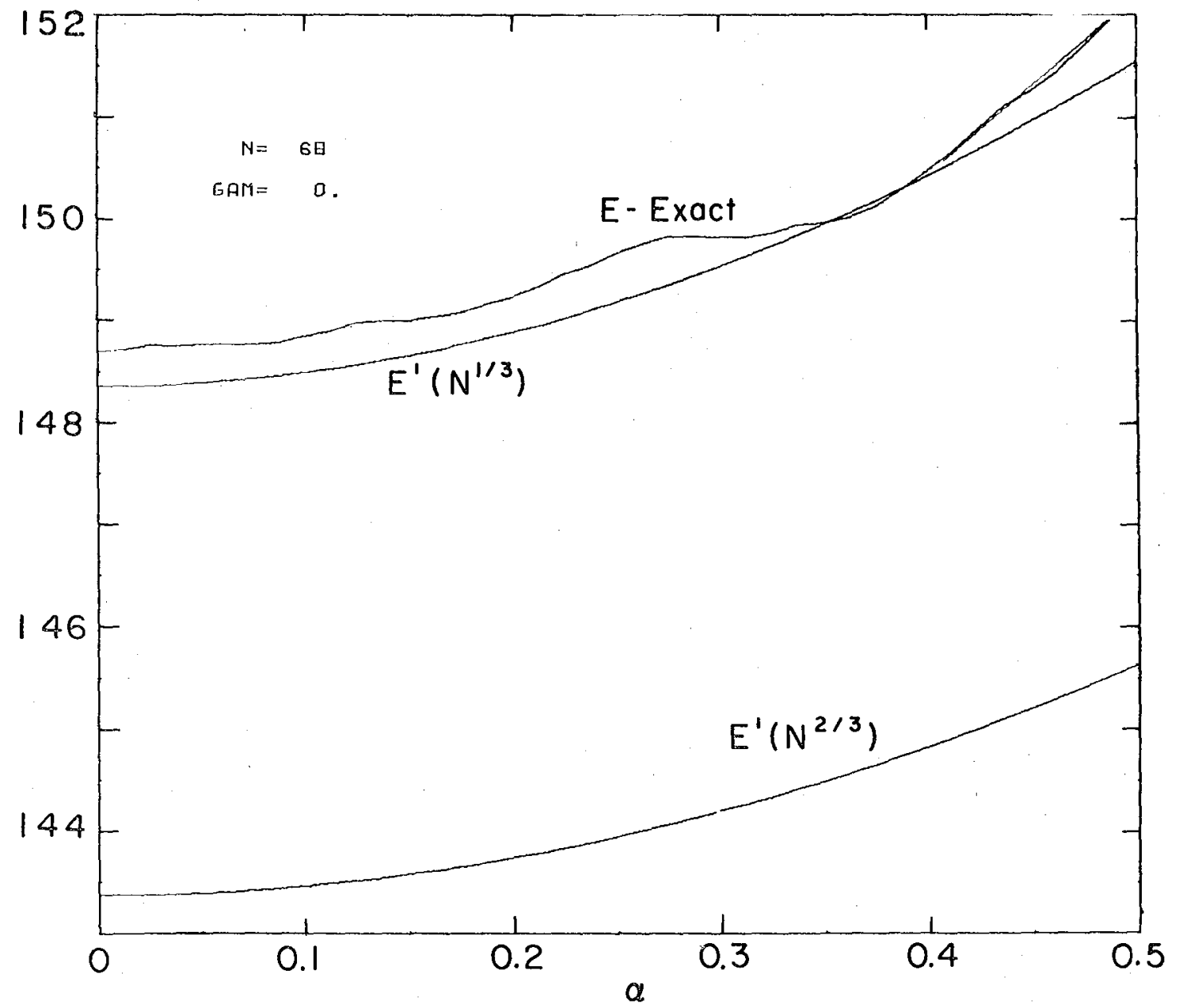

$X B L 693-2277$

Fig. 5b 
$-151-$

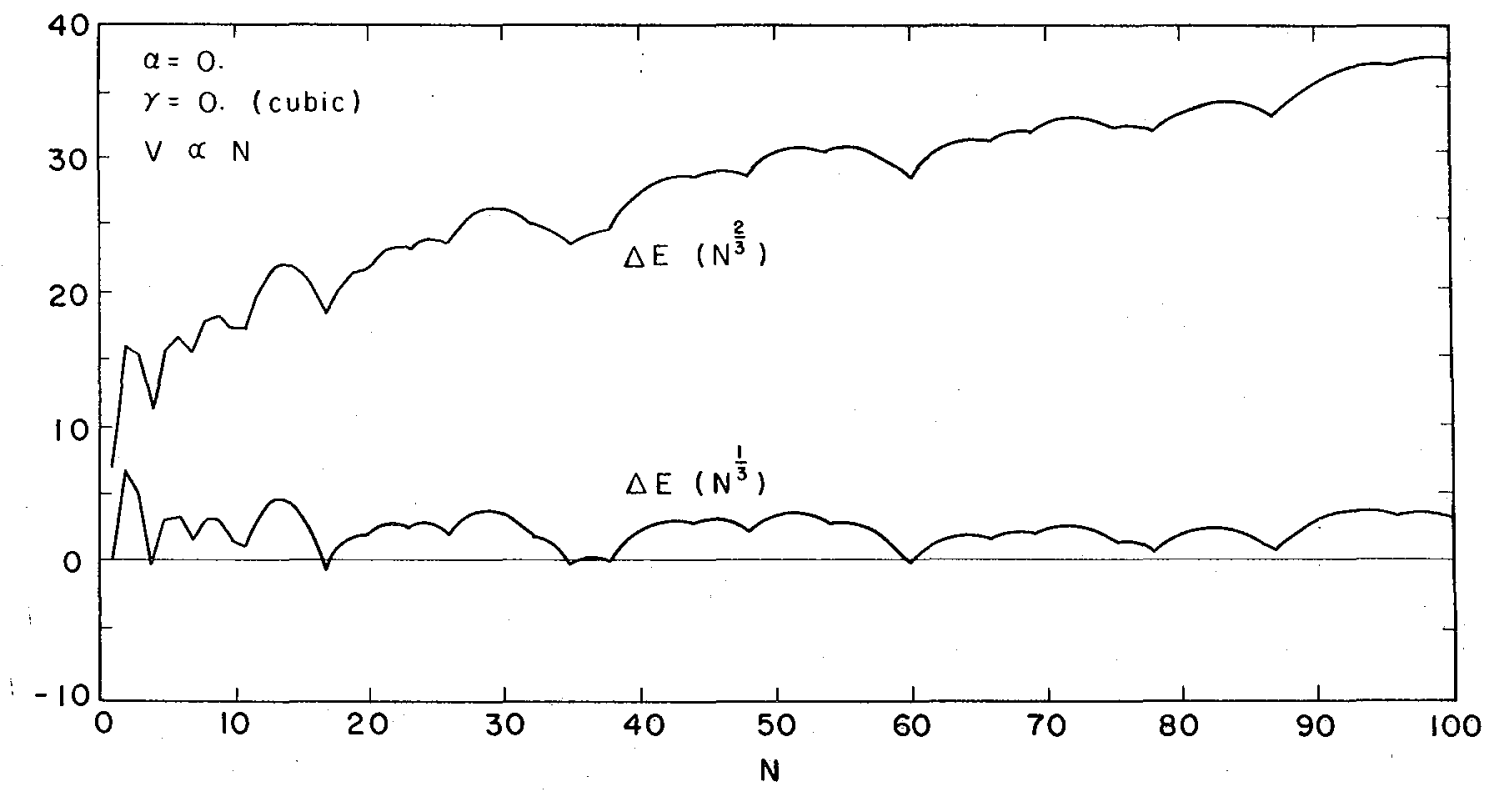

Fig. $6 a$ 


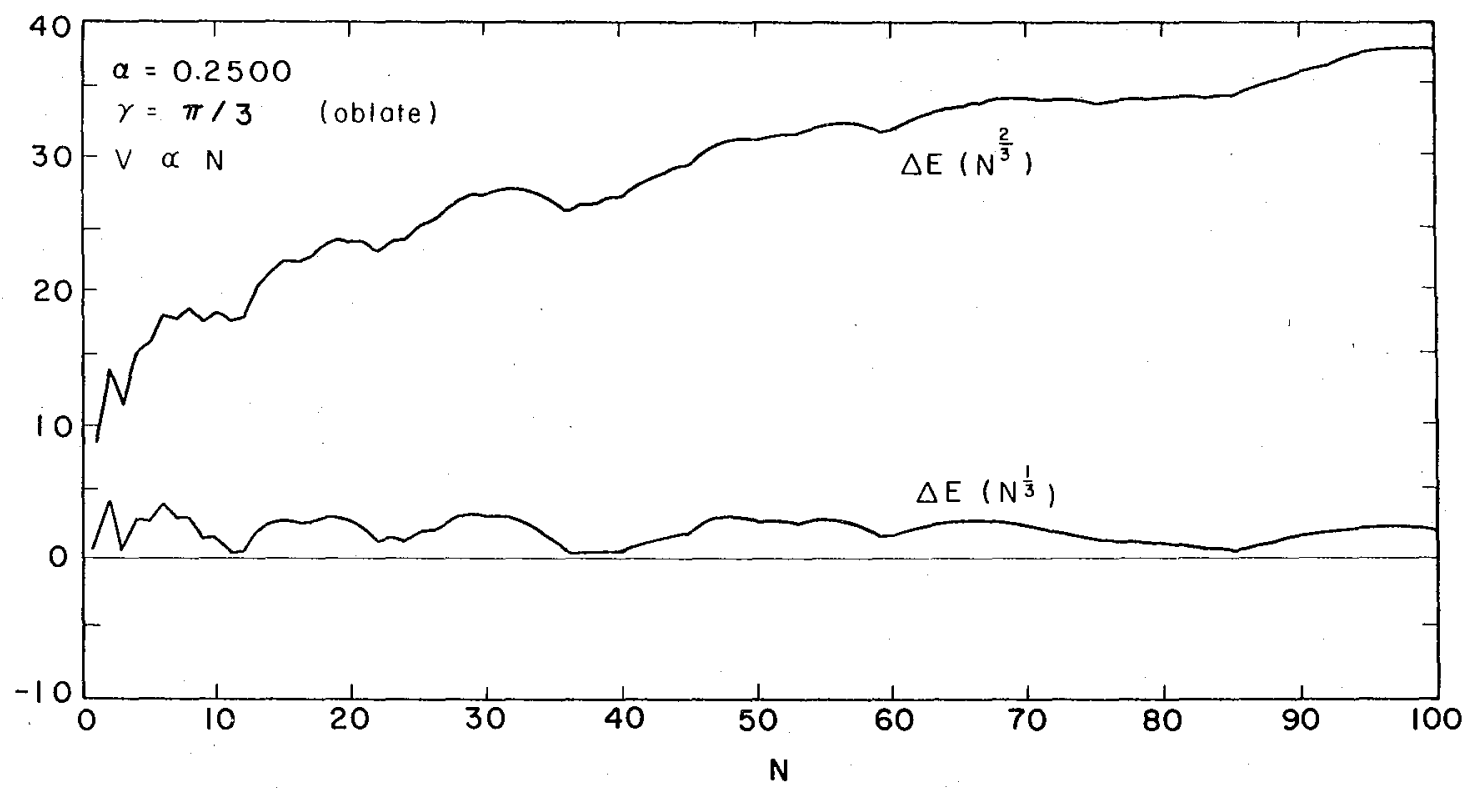

Fig. $6 b$ 


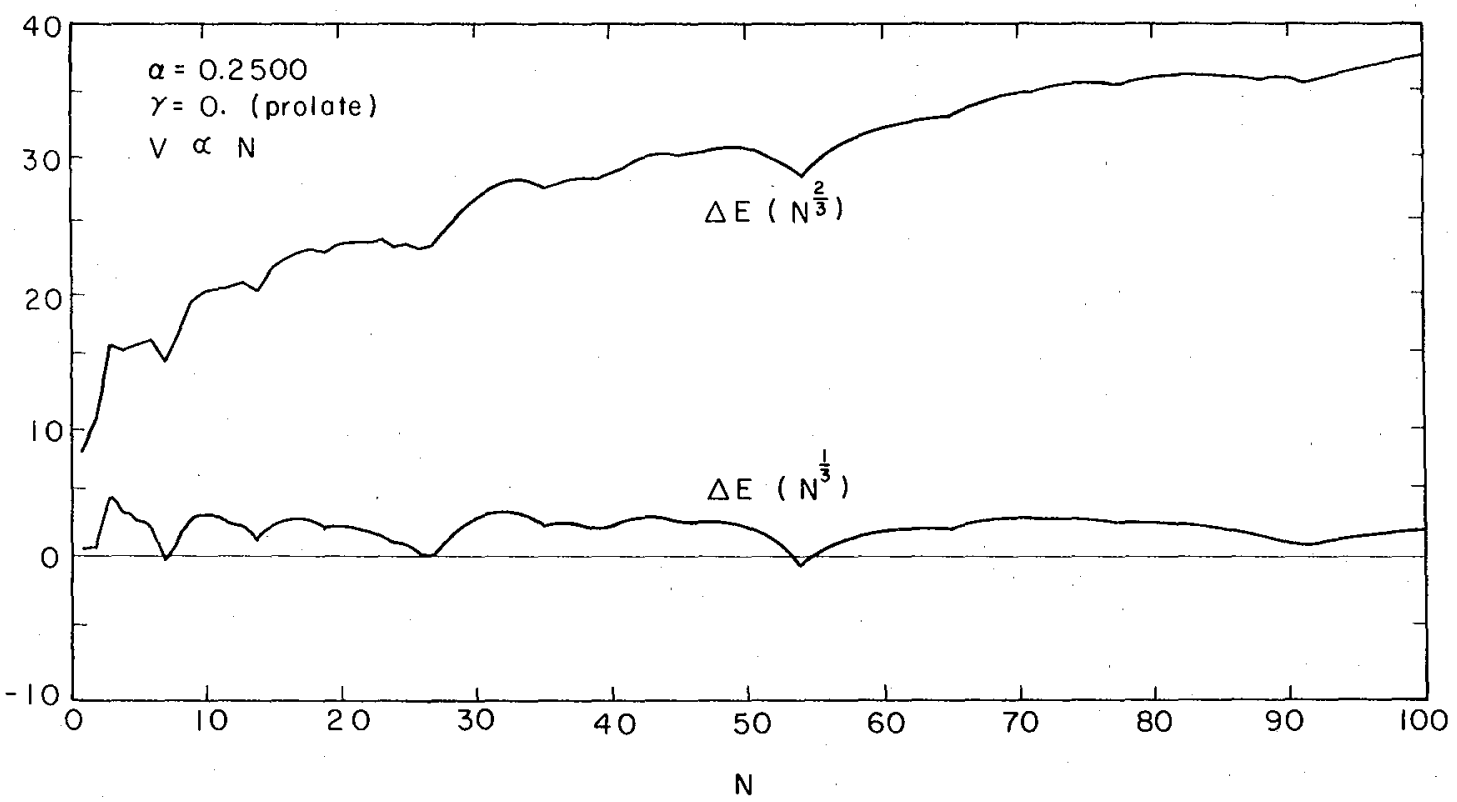

Fig. 6c 
$-154-$

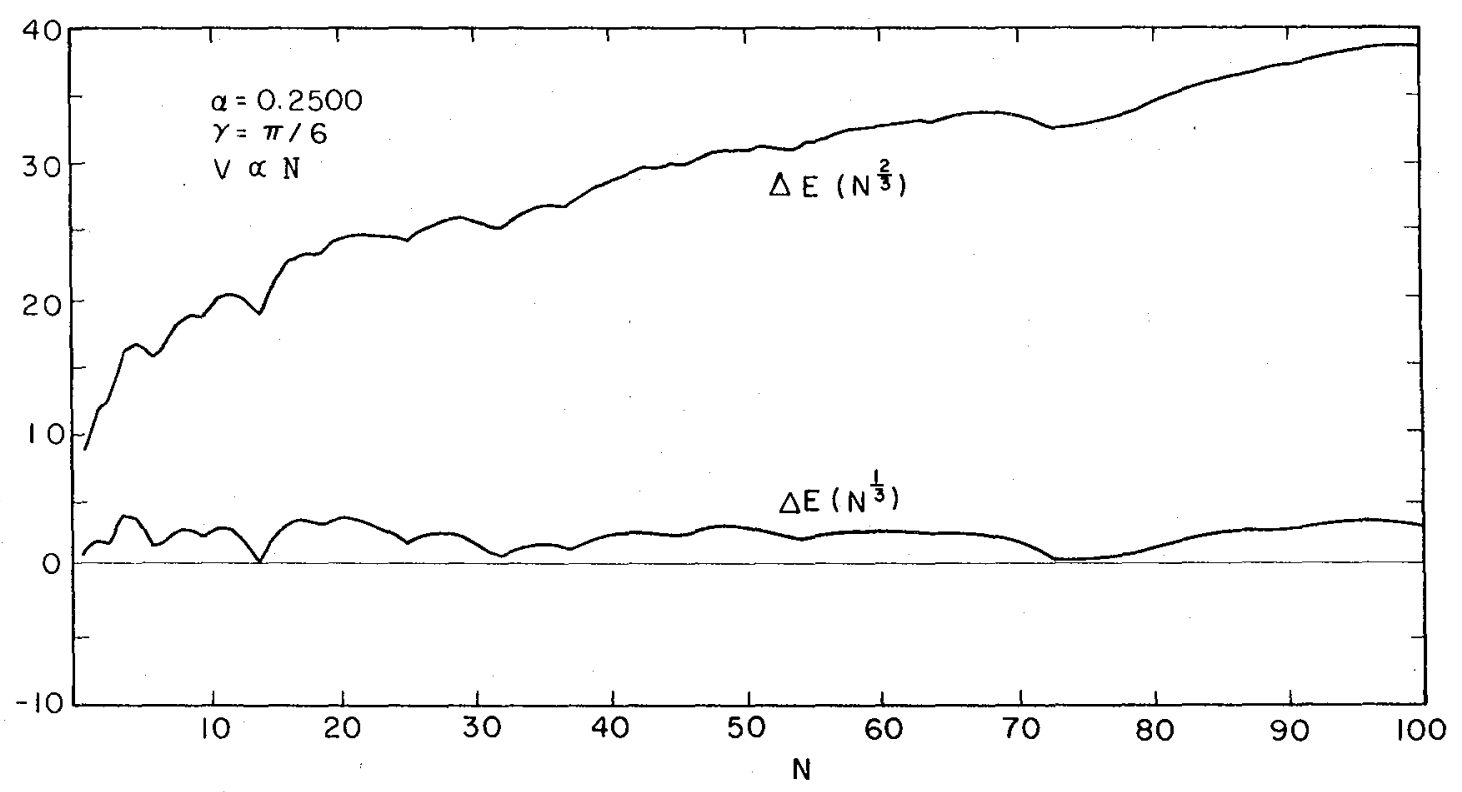

Fig. 6d 


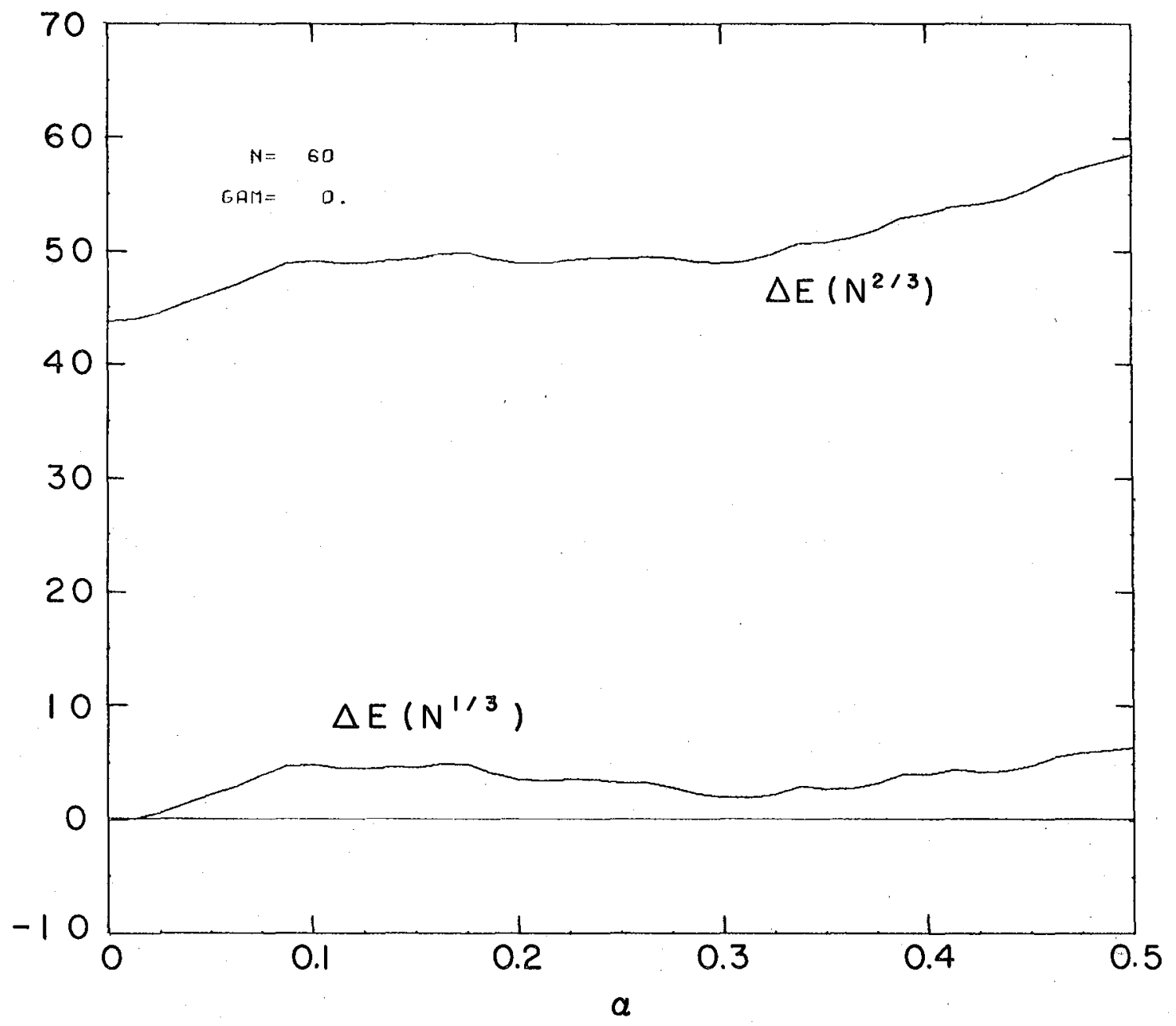

XBL $694-2466$

Fig. $7 a$ 
$-156-$

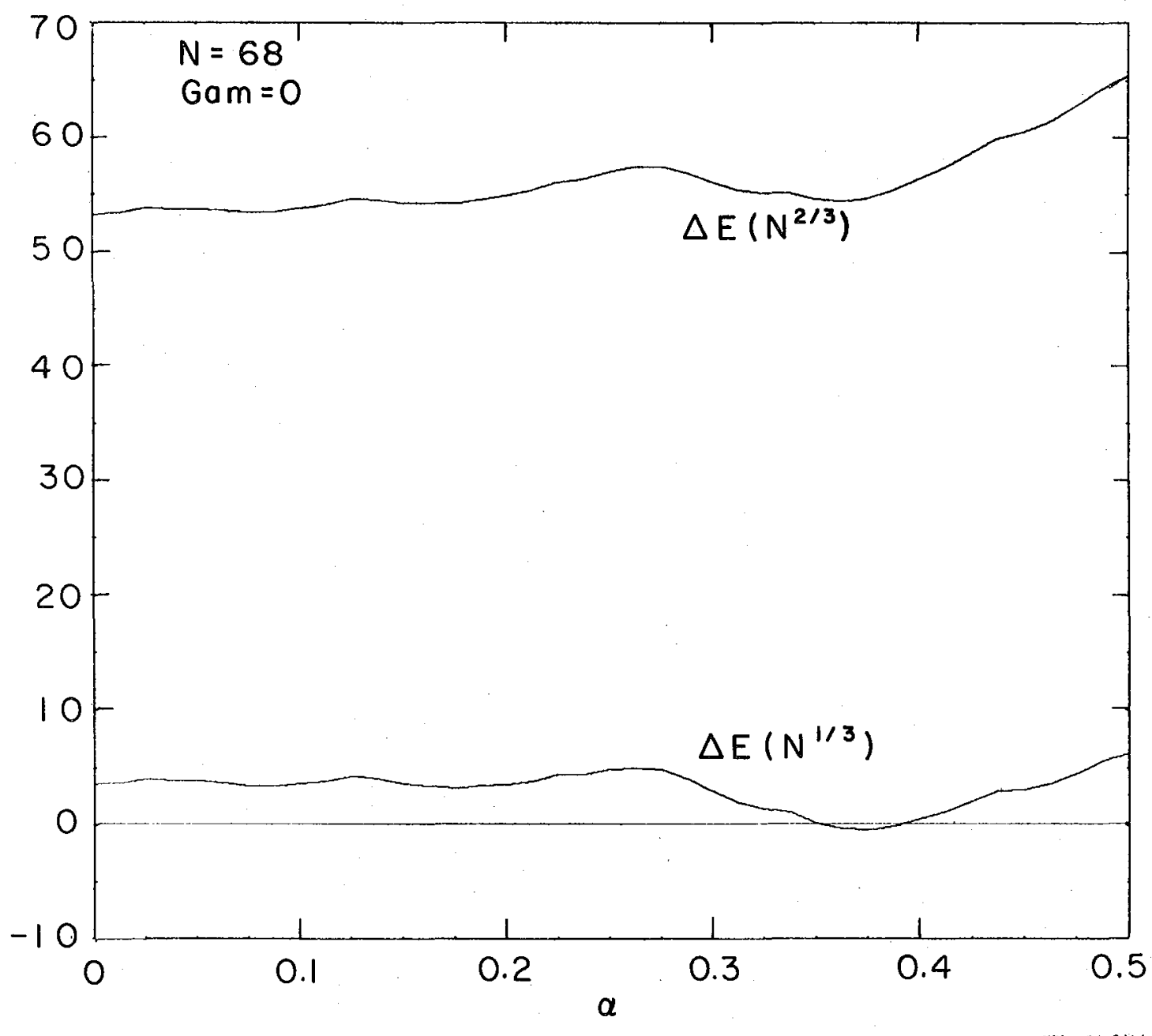

Fig. $7 \mathrm{~b}$ 


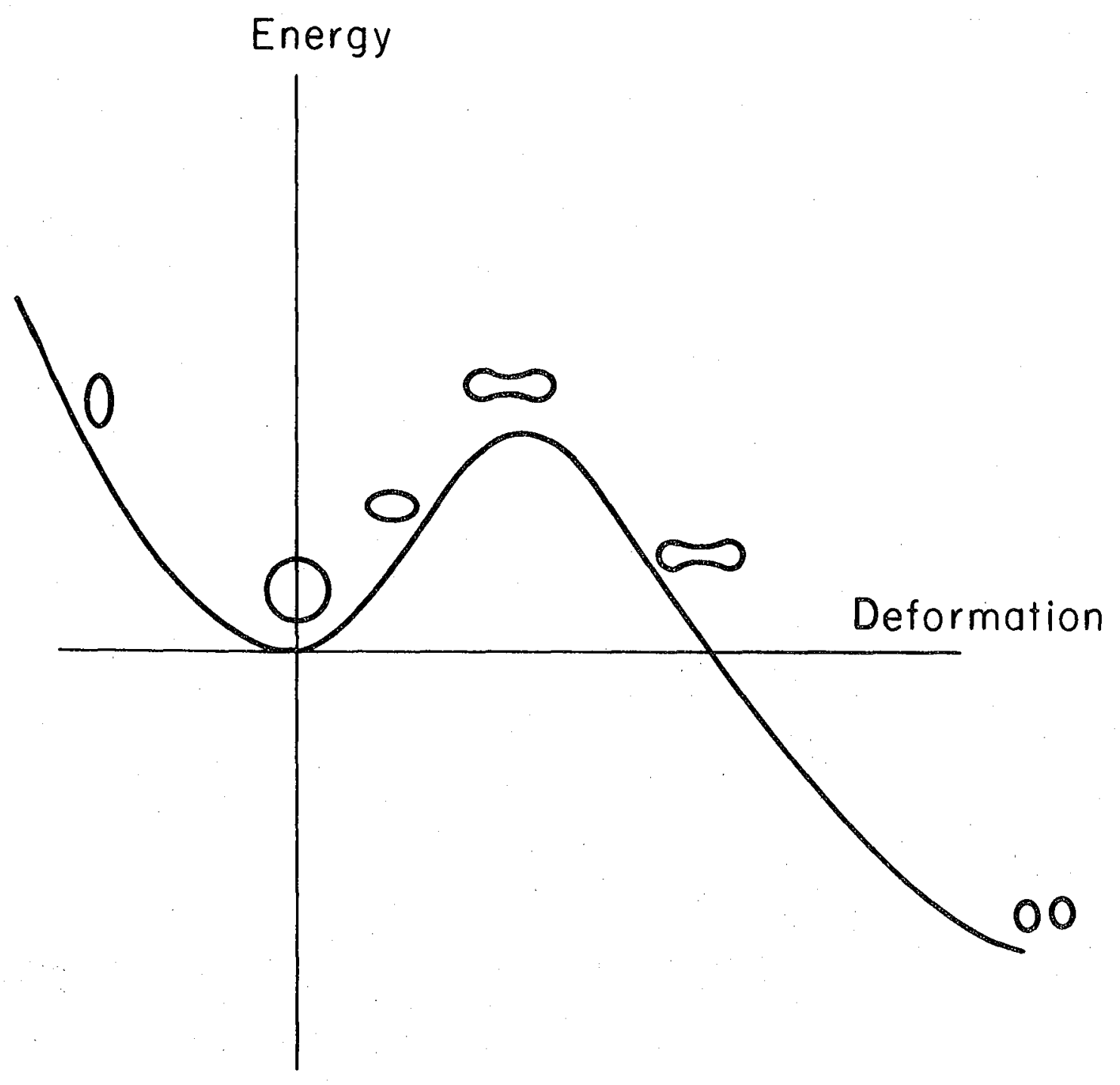

XBL $\quad 694-2467$

Fig. 8 


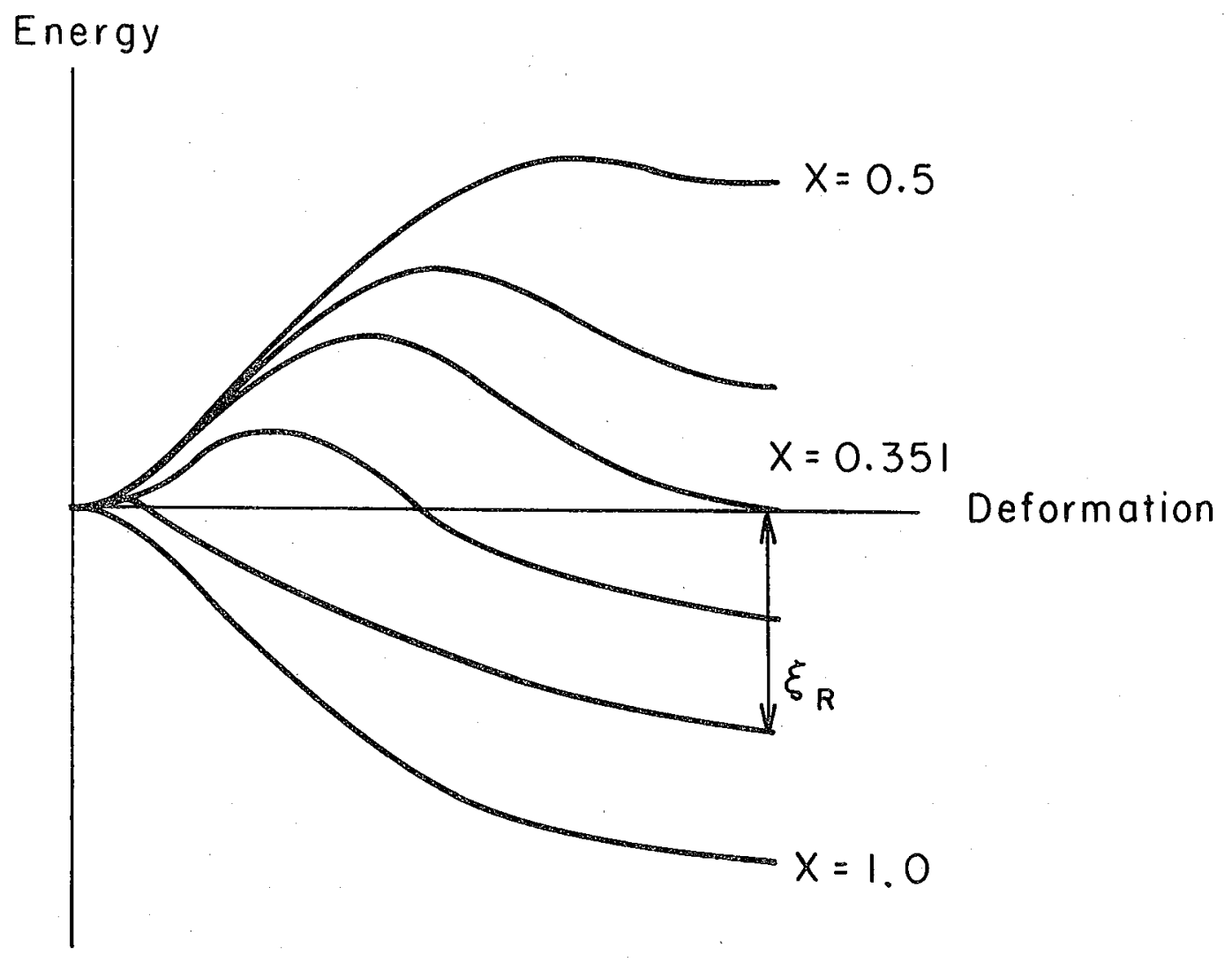

XBL 694-2457

Fig. 9 


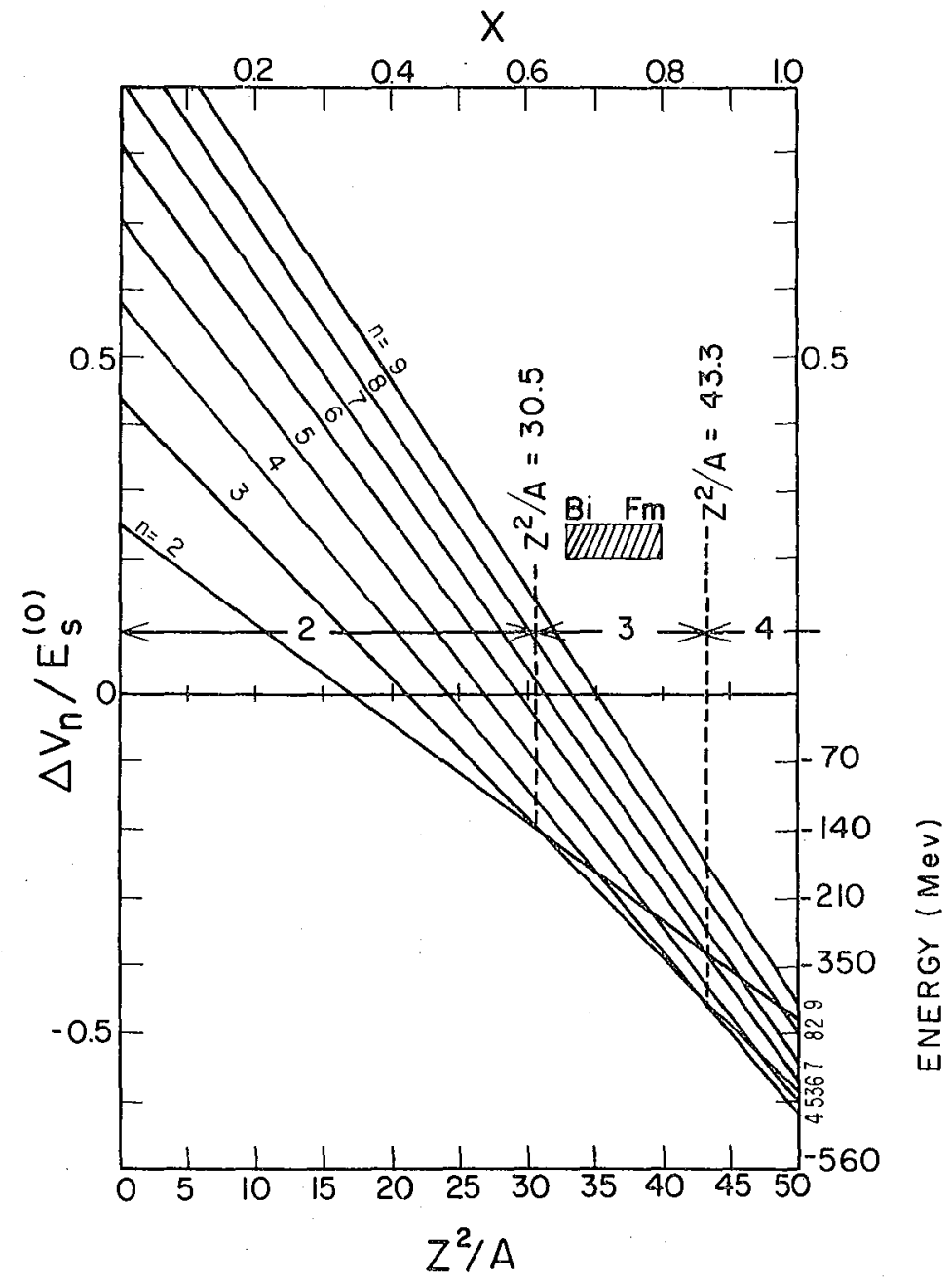

Fig. 10 
$-160-$

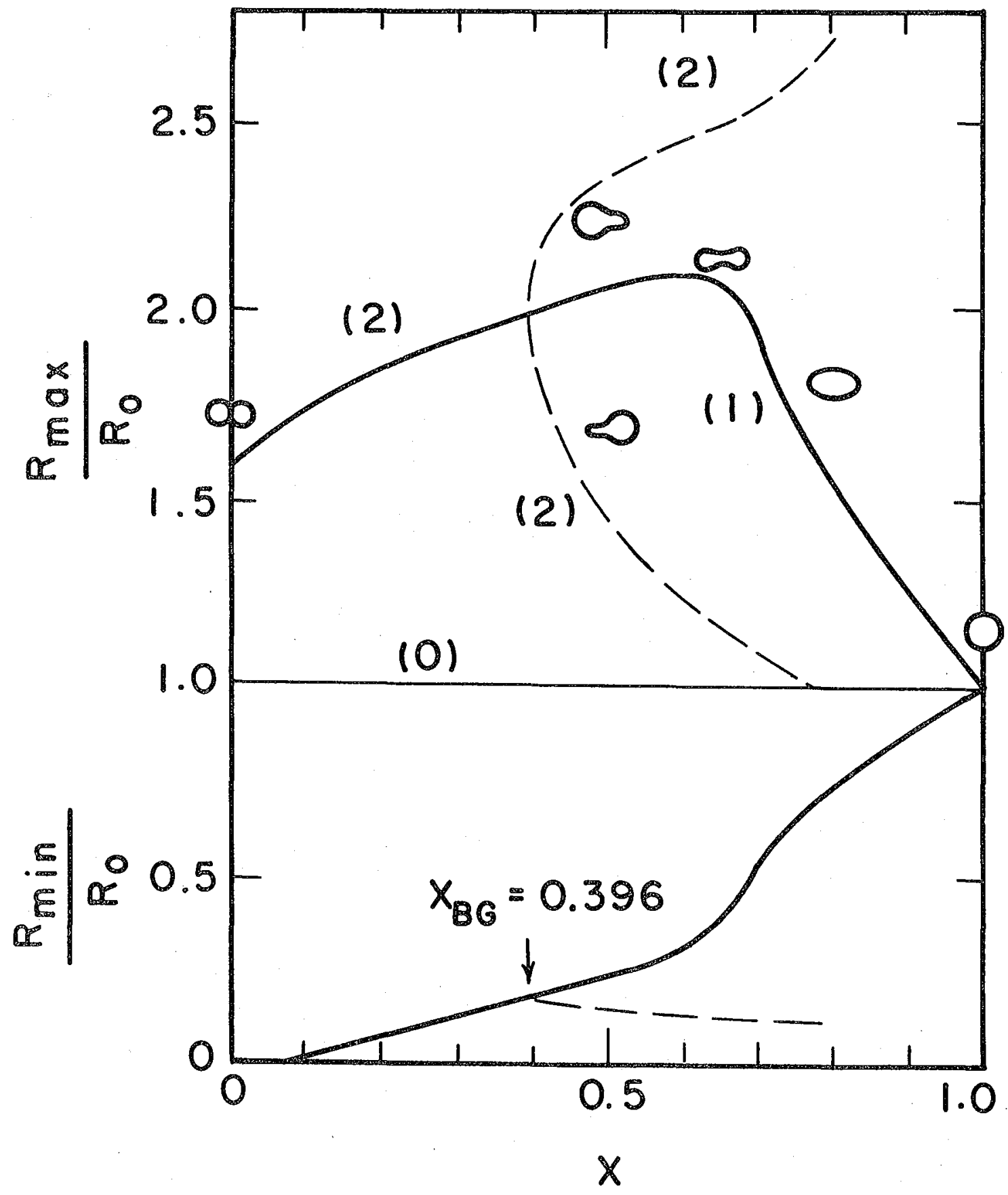

XBL 694-2461

Fig. 11 


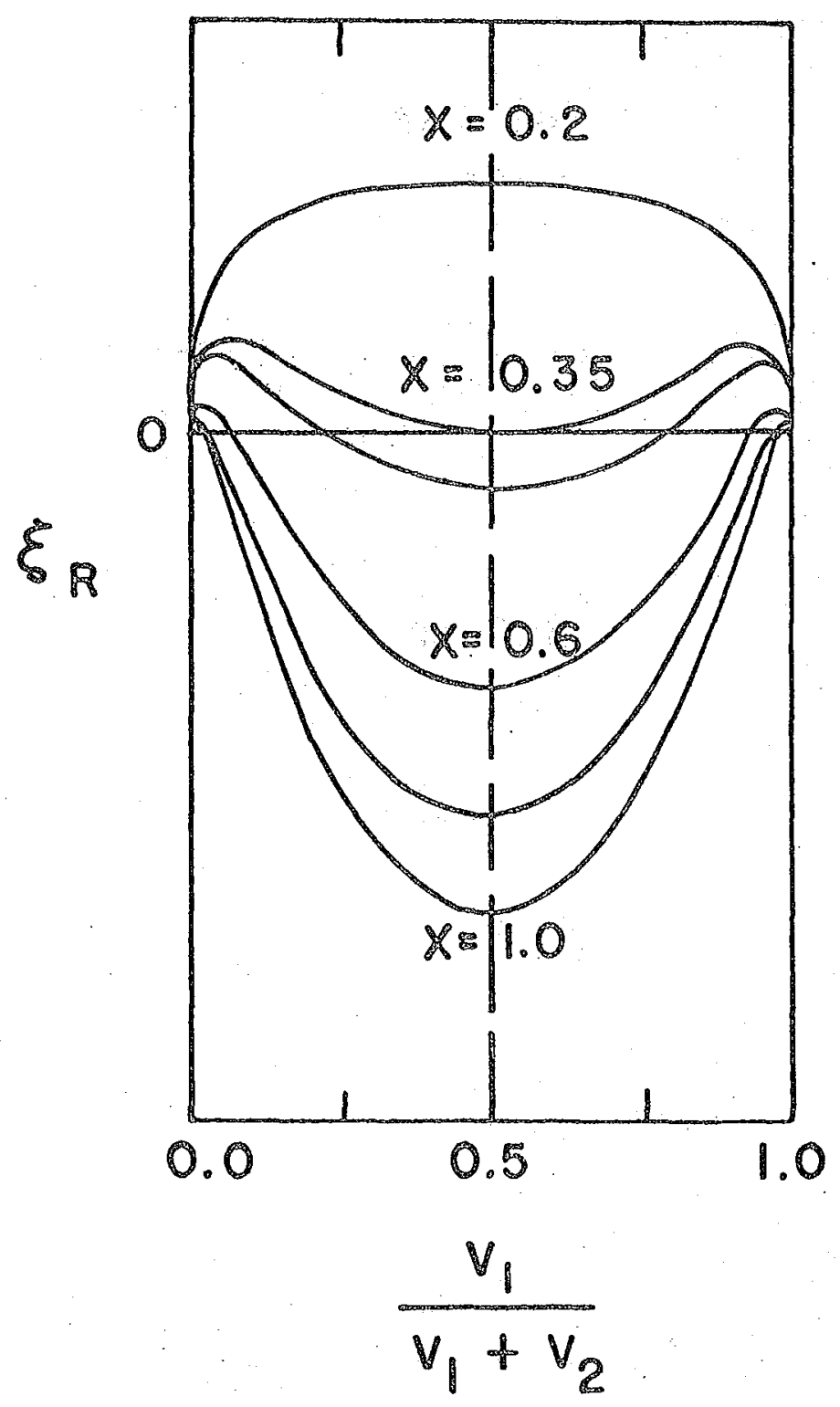

XBL $\quad 694-2458$

Fig. 12 


\section{$-162-$}

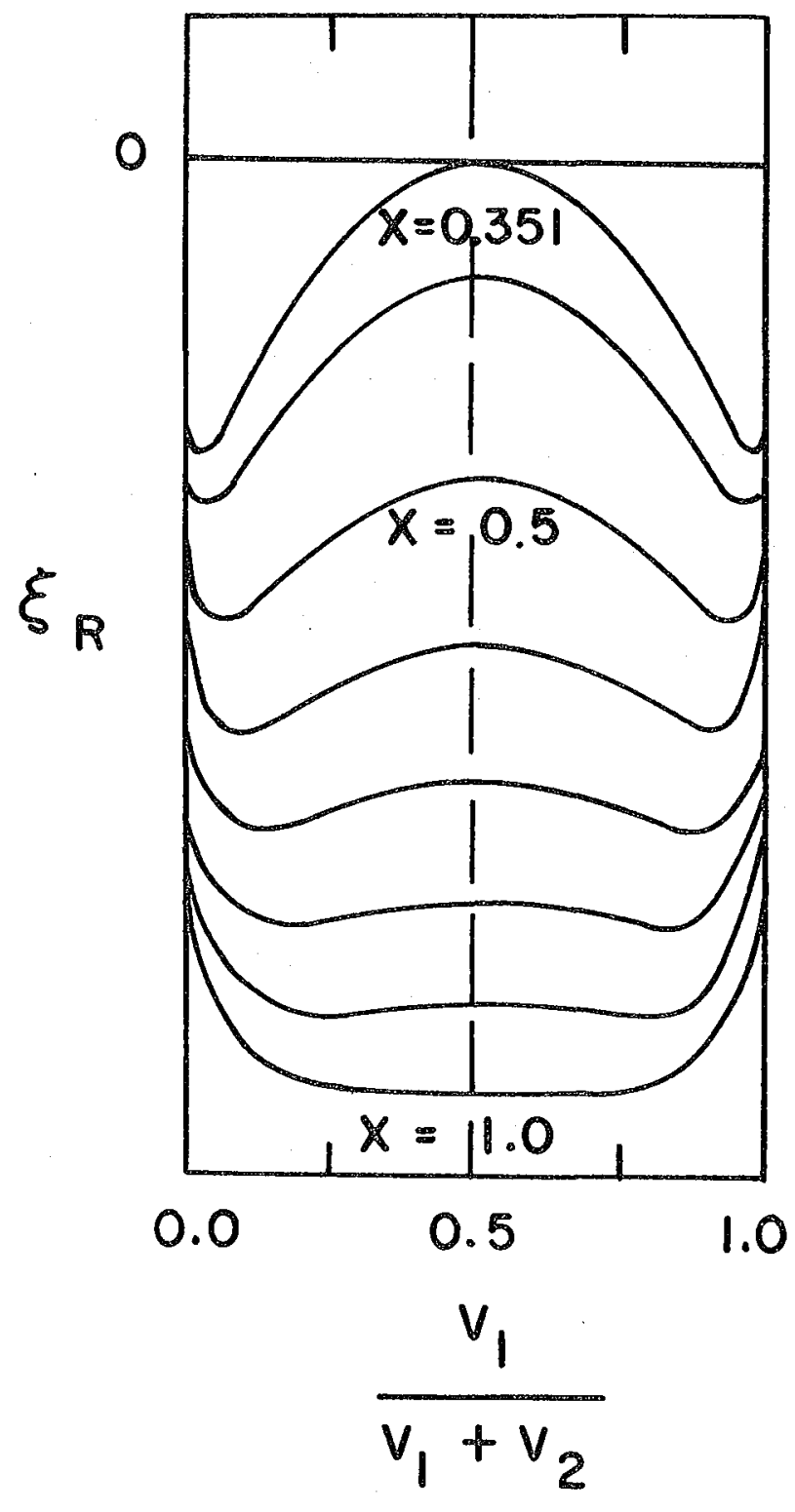

XBL 694-2459

Fig. 13 


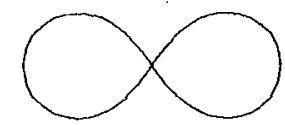

$s=0$.
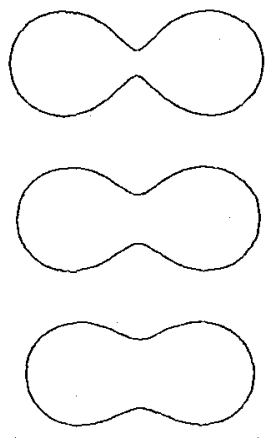

$s=\quad .3000$
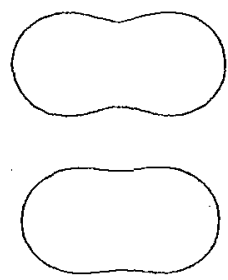

$s=\quad .5000$
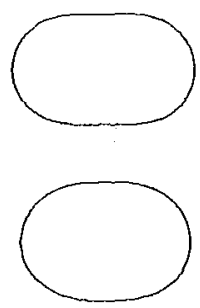

$s=\quad .7000$

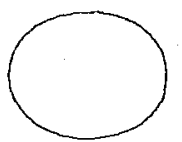

$S=.0000$

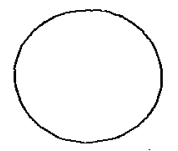

$S=\quad .9000$

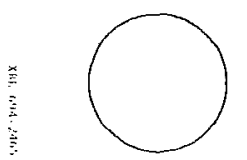

$S=1.0000$

Fig. 14 


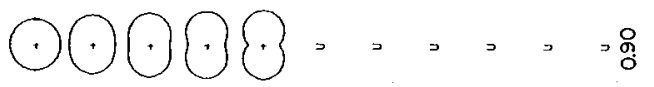

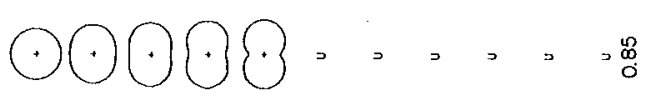

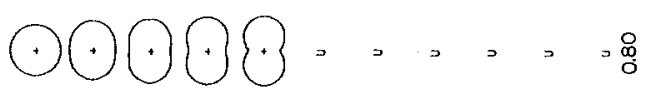

OPOP\}? = = =

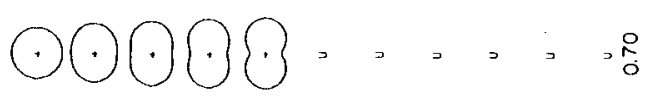

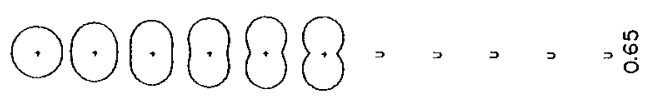

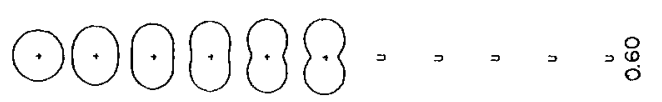

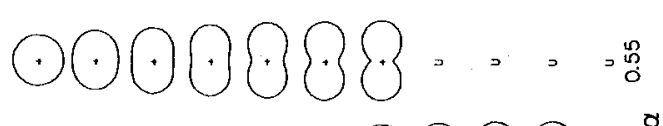

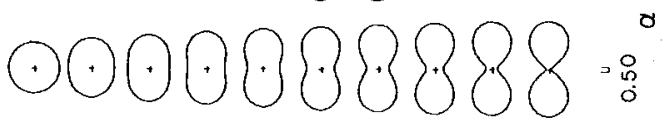

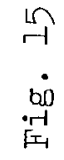

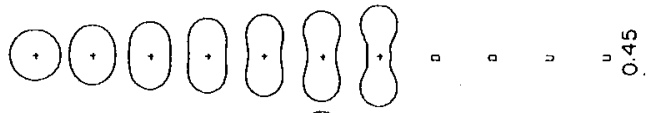

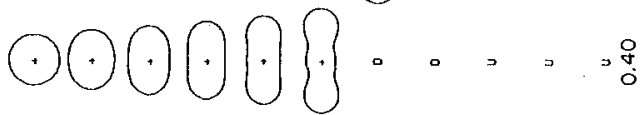

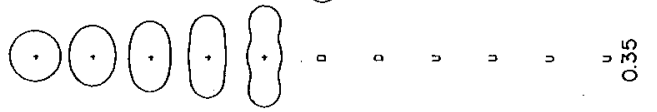

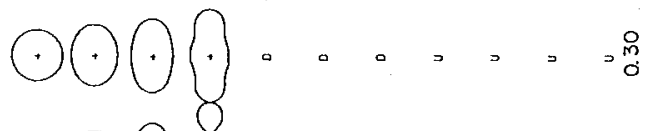

$\odot \odot \bullet\left\{\begin{array}{l}0 \\ 0\end{array}\right.$

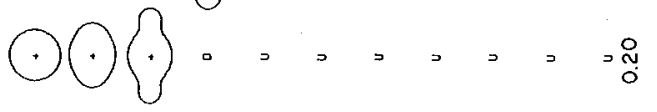

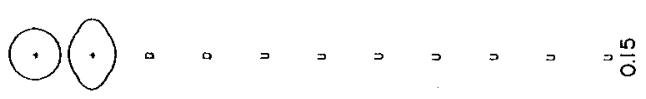

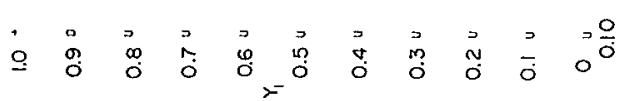




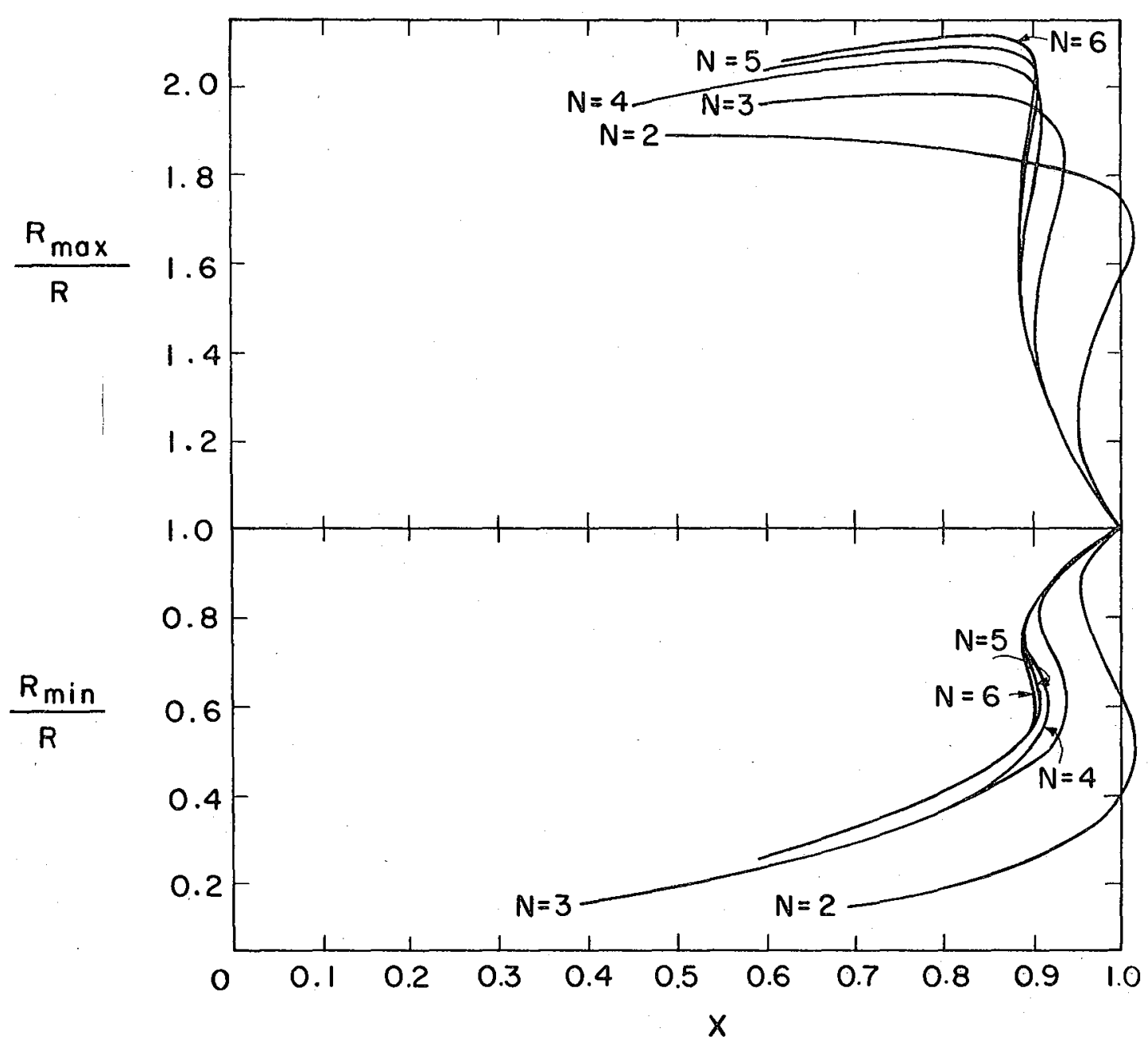

XBL696- 2927

Fig. 16 


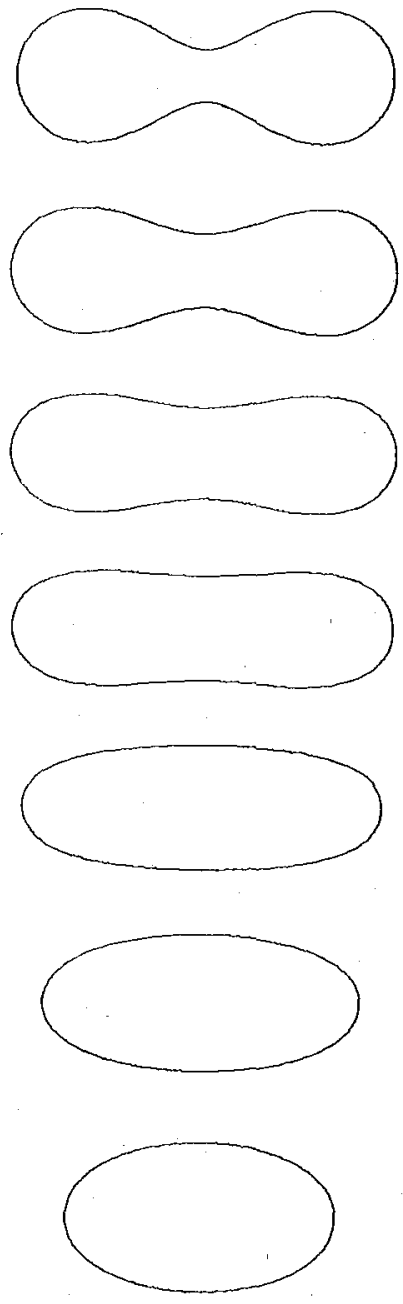

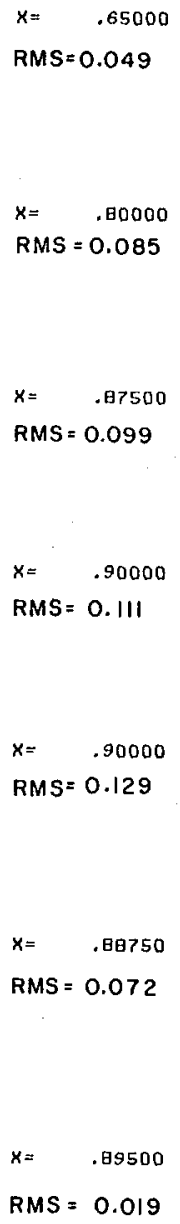

XEL694-2518

Soddle point shopes

6 points charges model

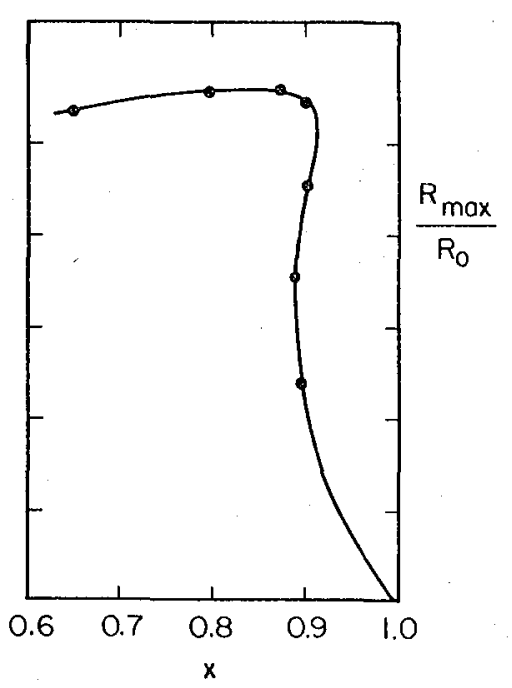

Fig. 17 


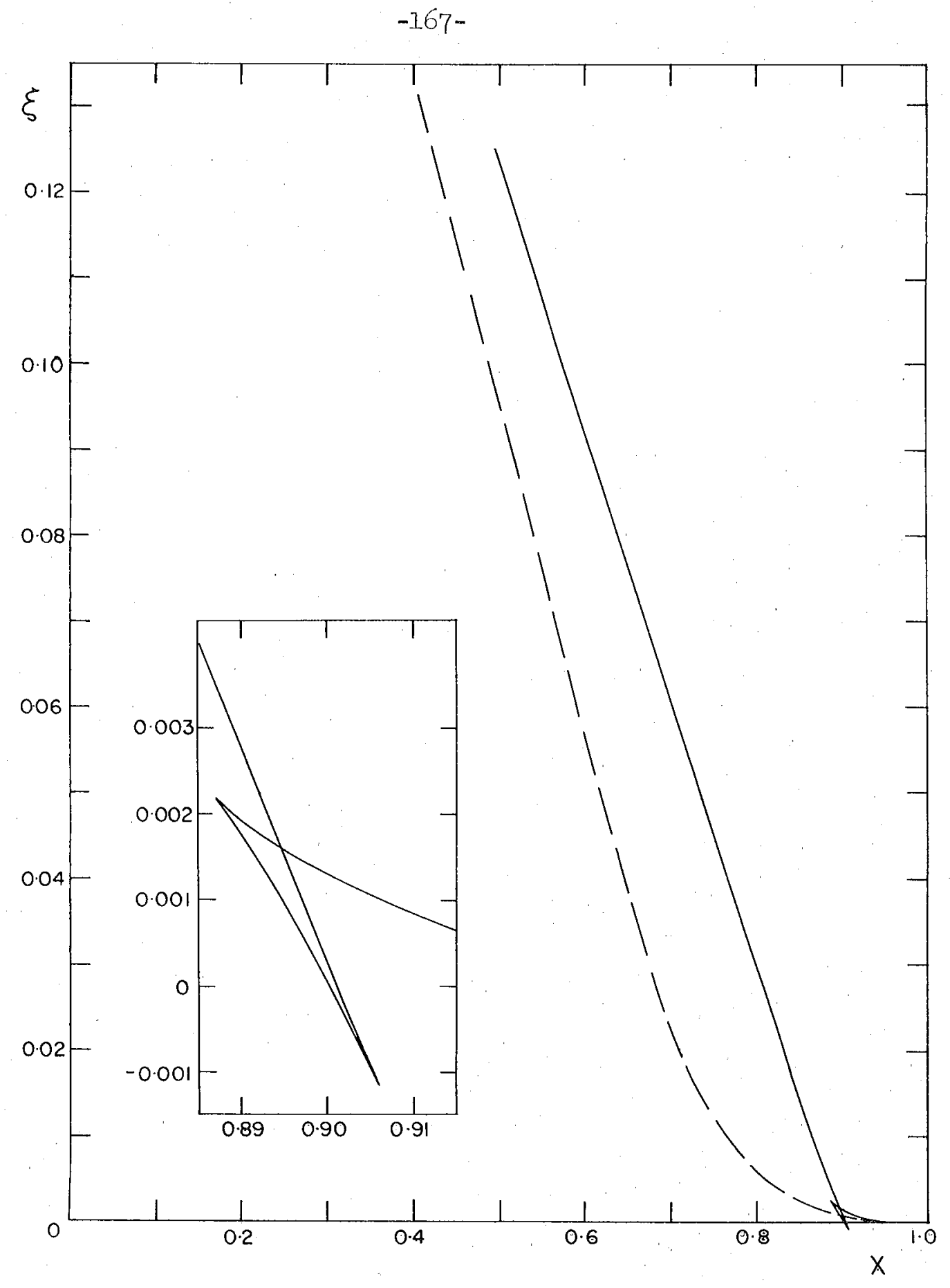

Fig. 18 
$-168-$

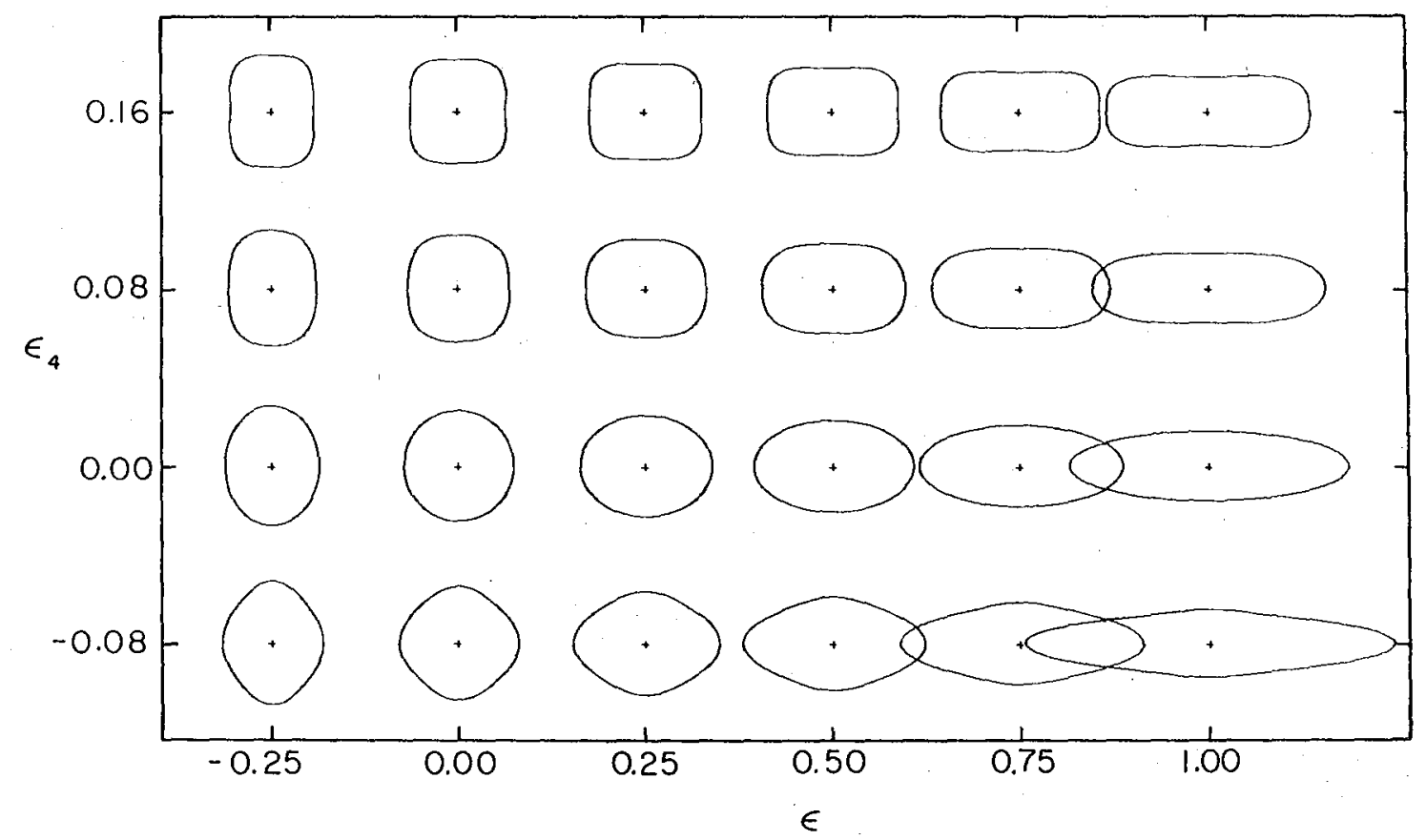

XBL6811-7120

Fig. 19 


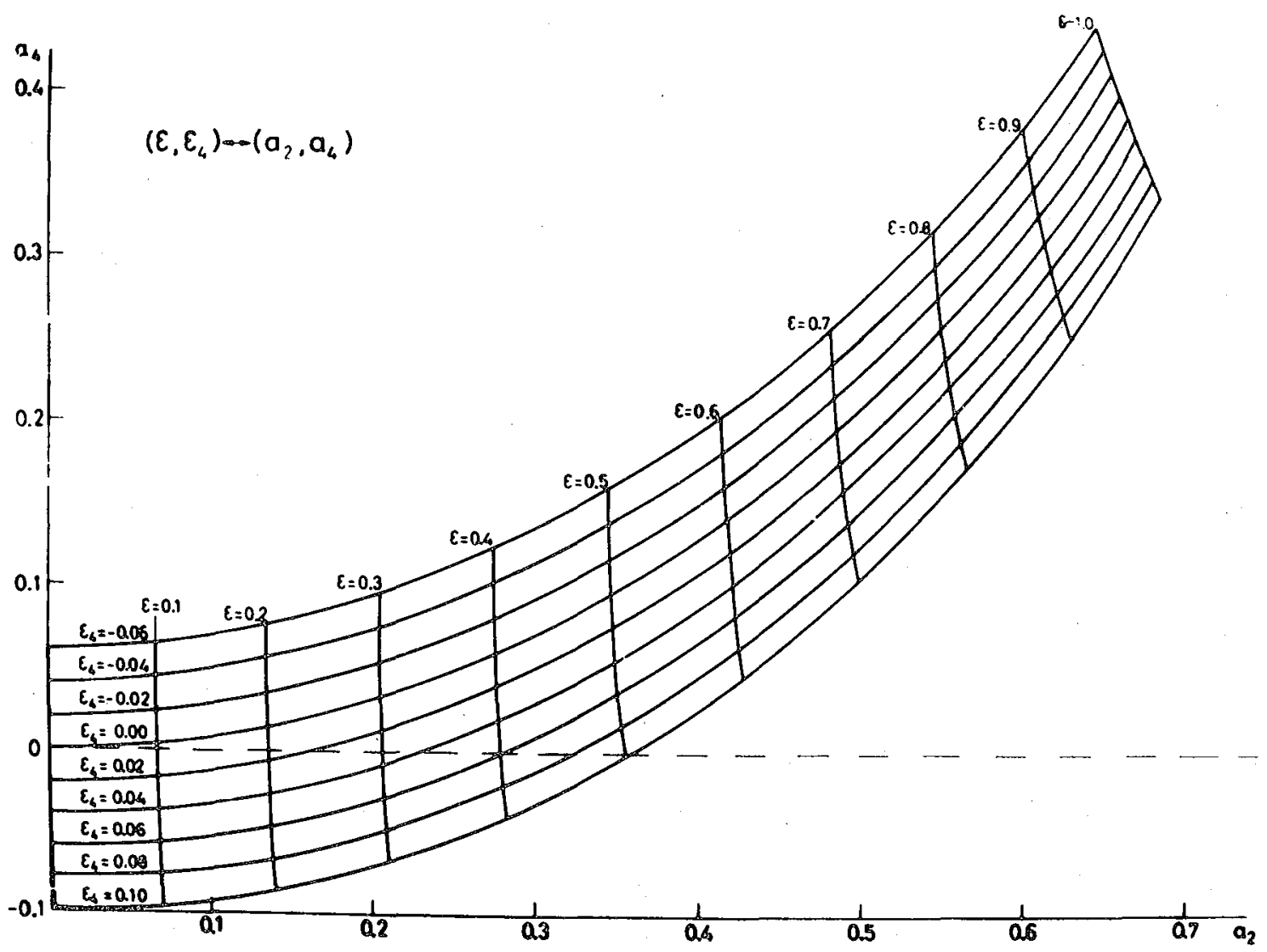

XBL 678-4633

Fig. 20 
Protons

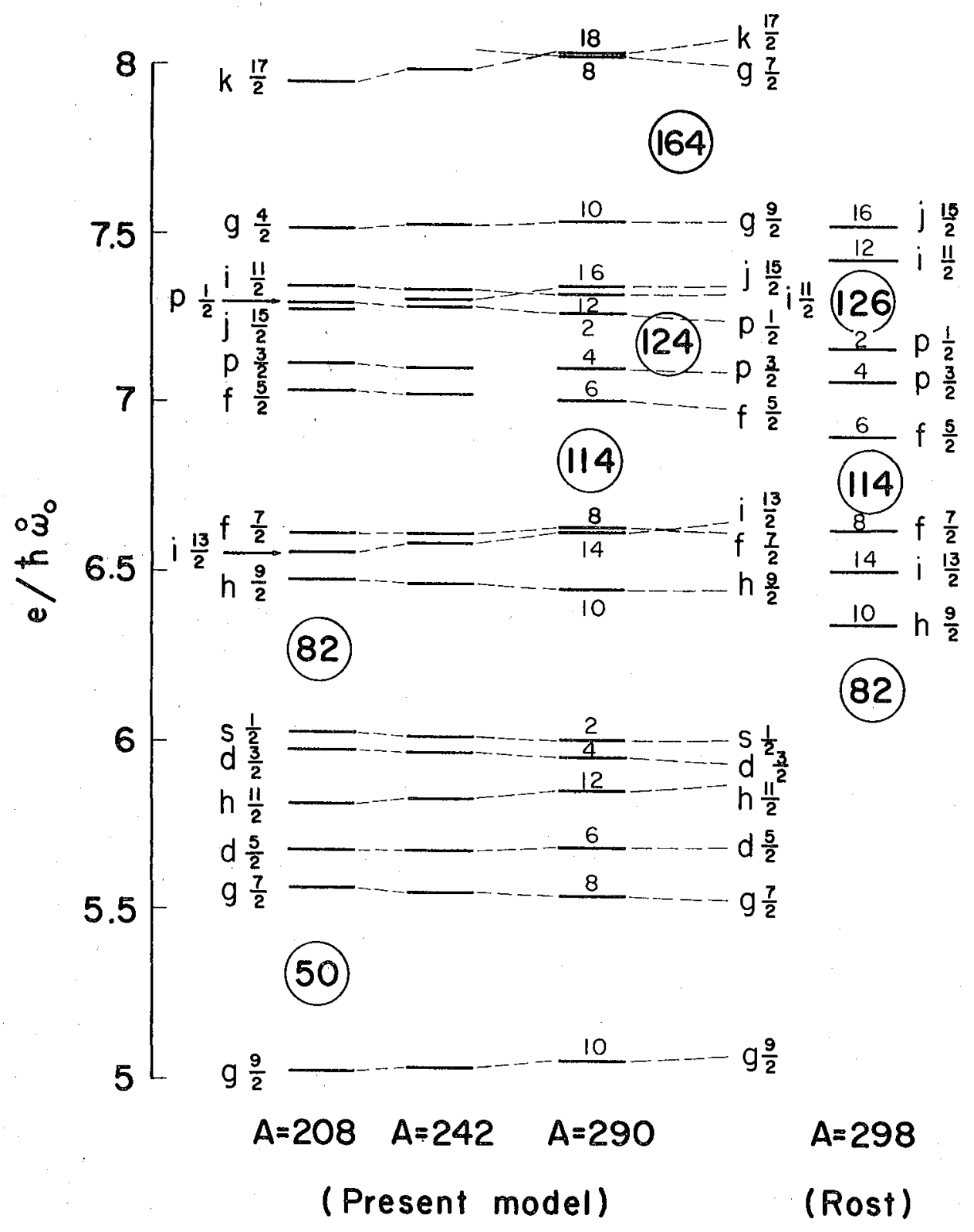

$X B L 682-1807$

Fig. 21 
Neutrons

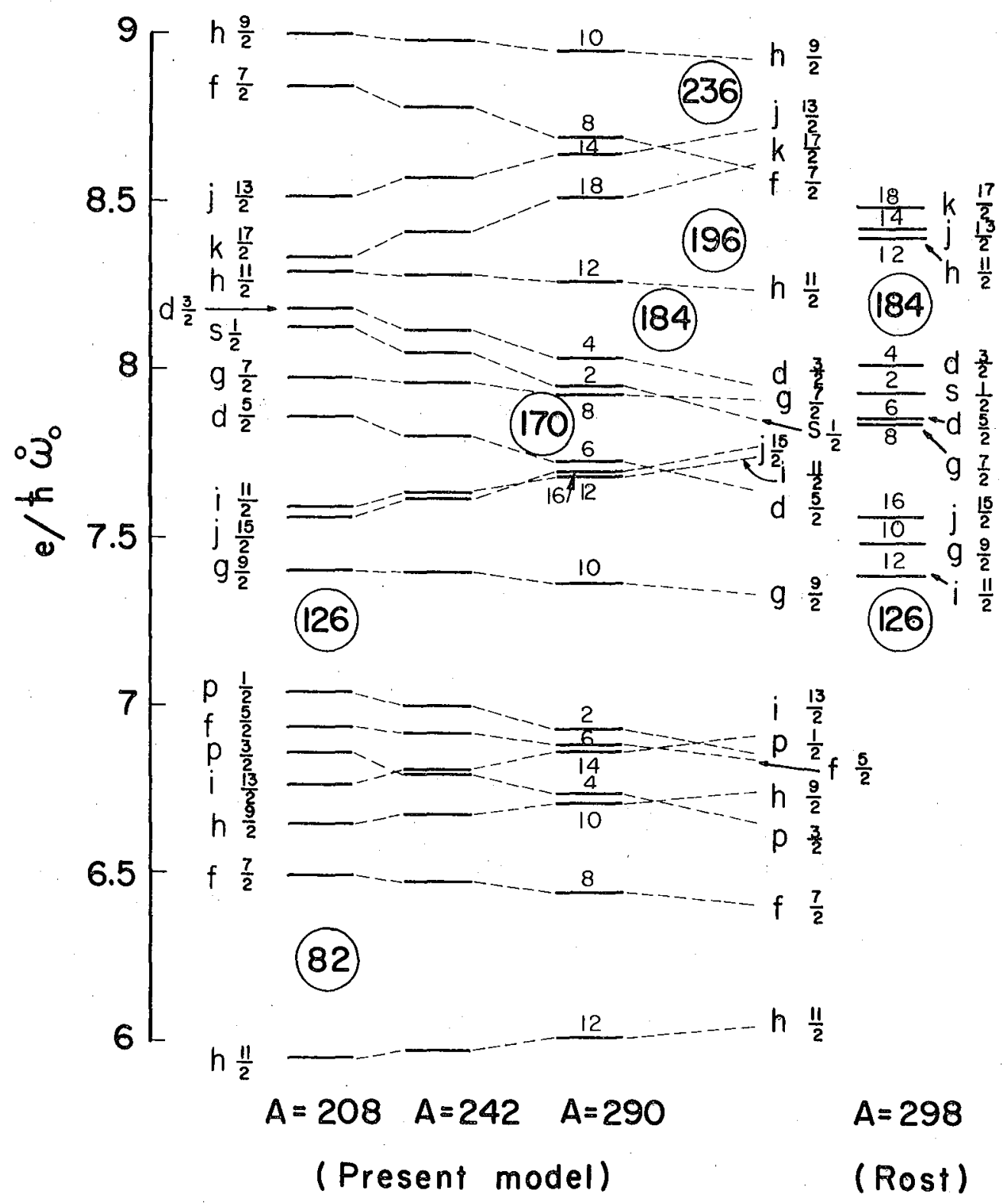

$X B L 682-1808$ 


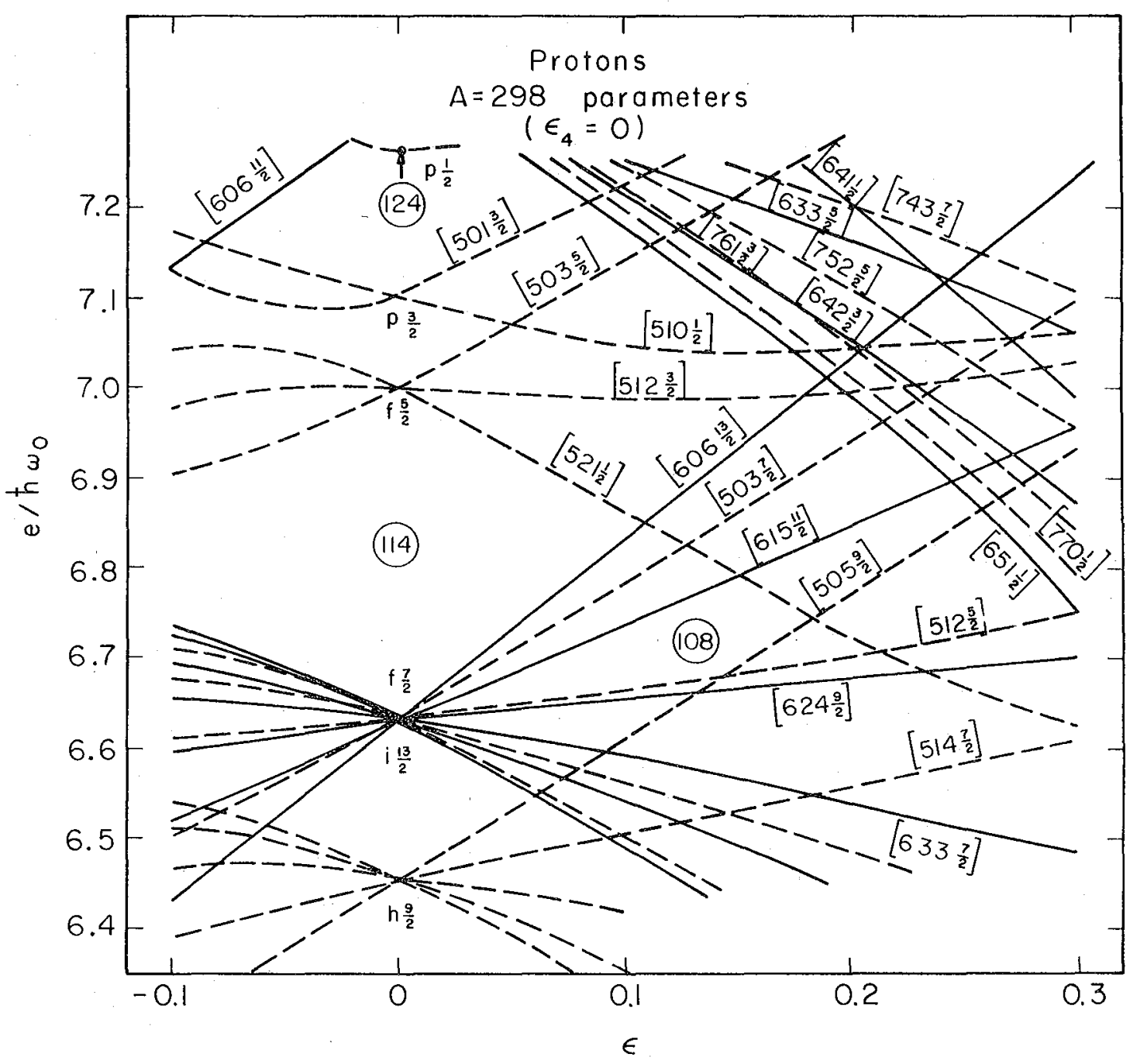

XBL687-3434

Fig. 23 


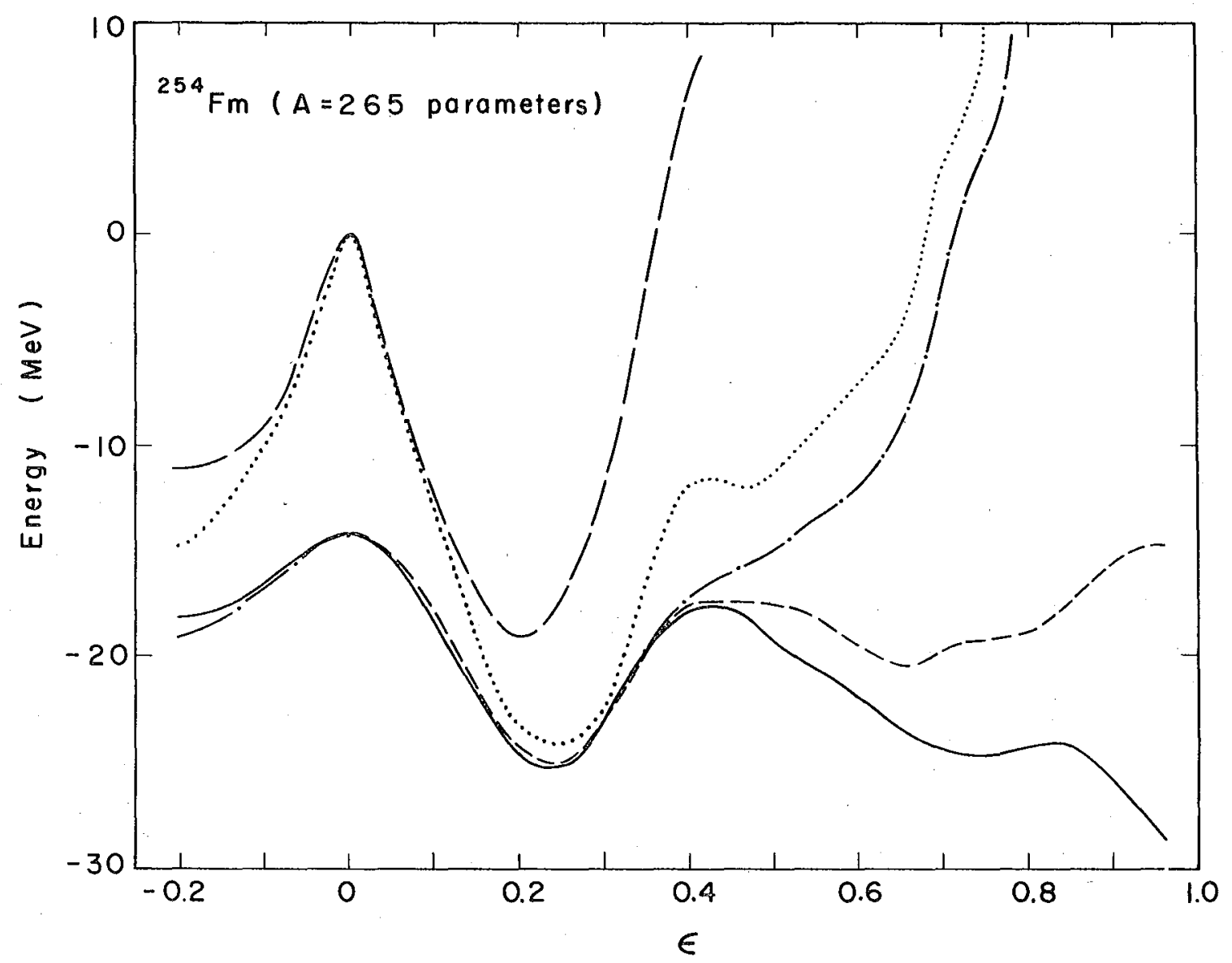

XBL6B7-3436

Fig. 24 


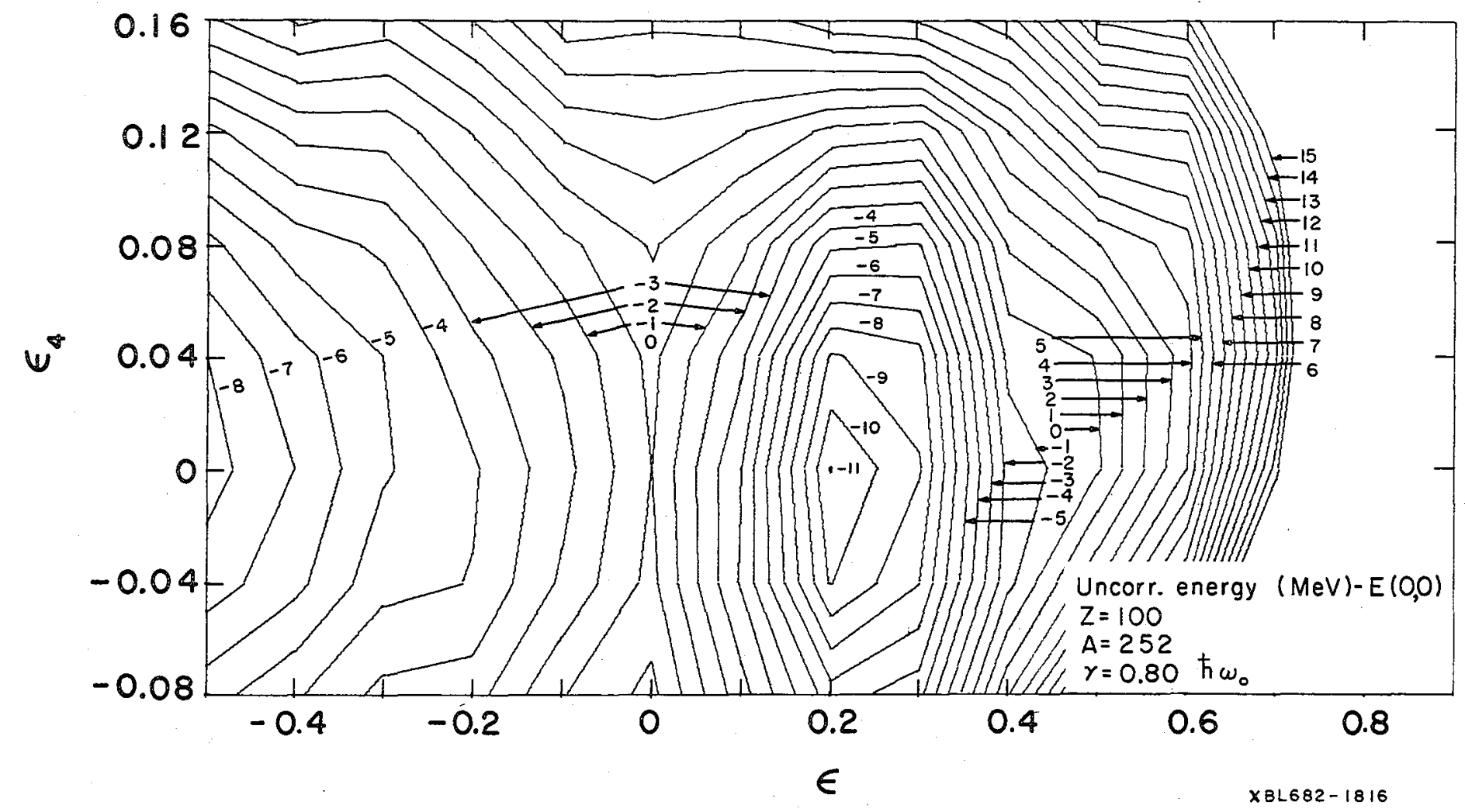




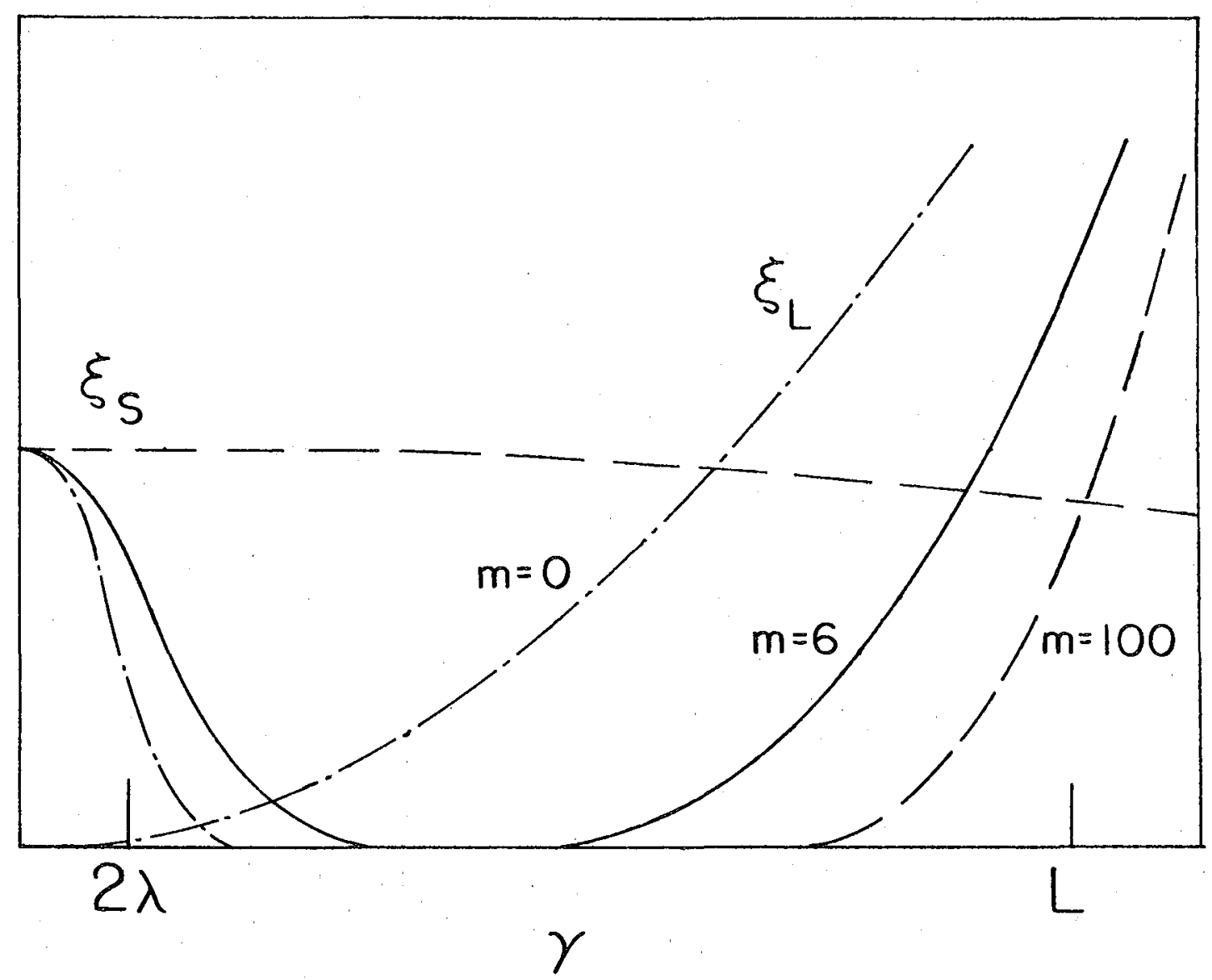

XBL 695-583

Fig. 26 


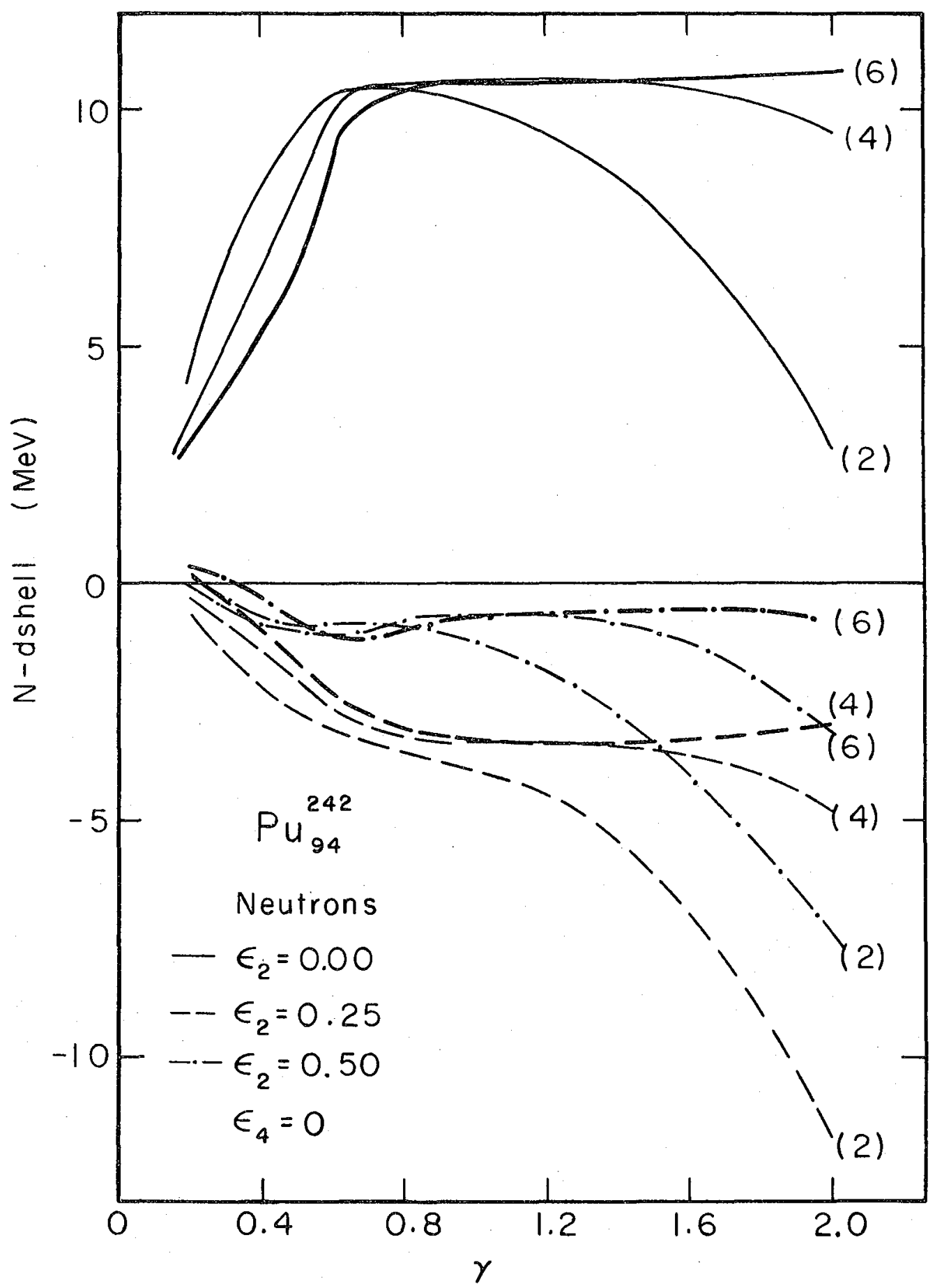

$X B L 686-2936$ 


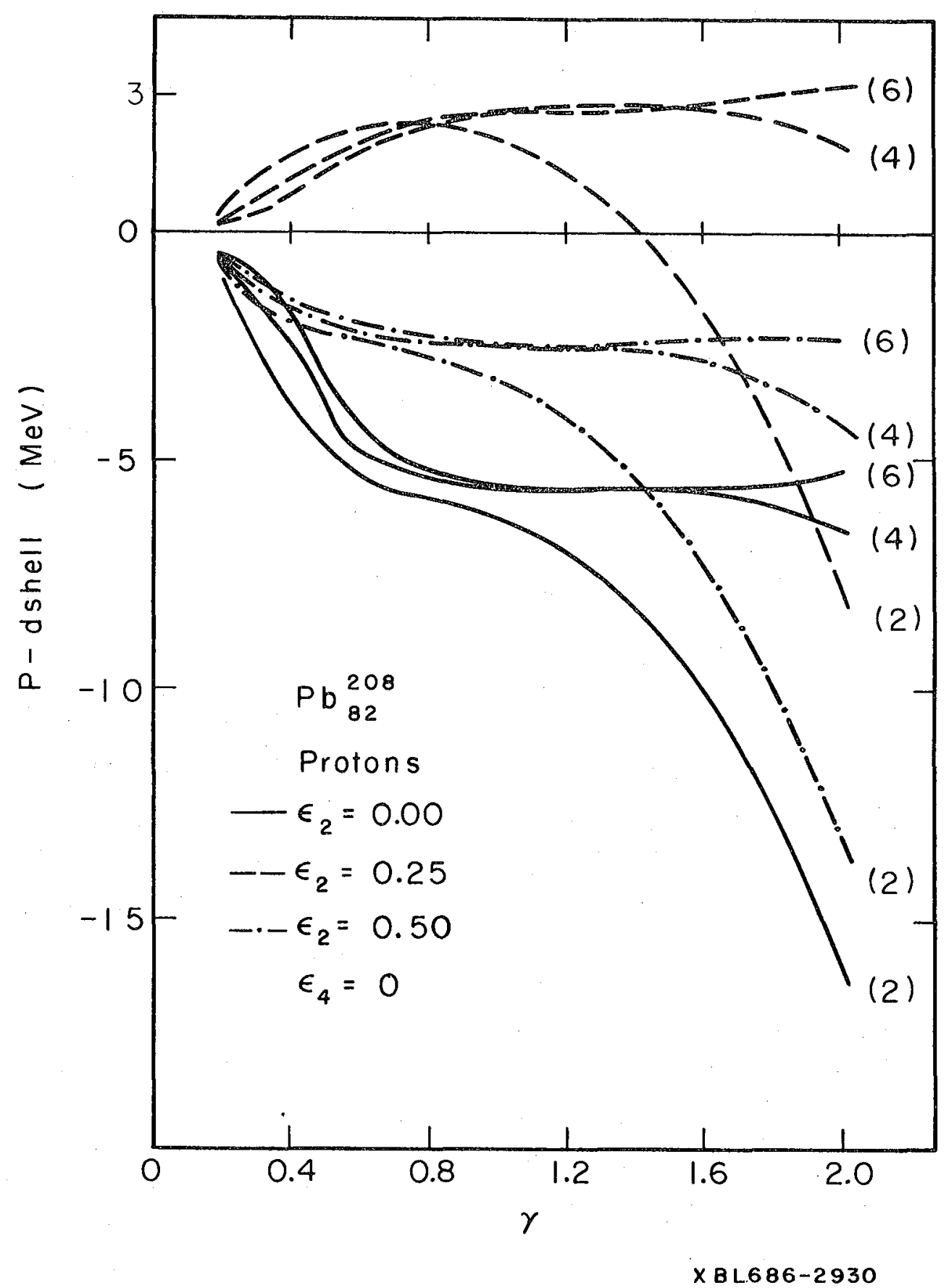

Fig. 28 


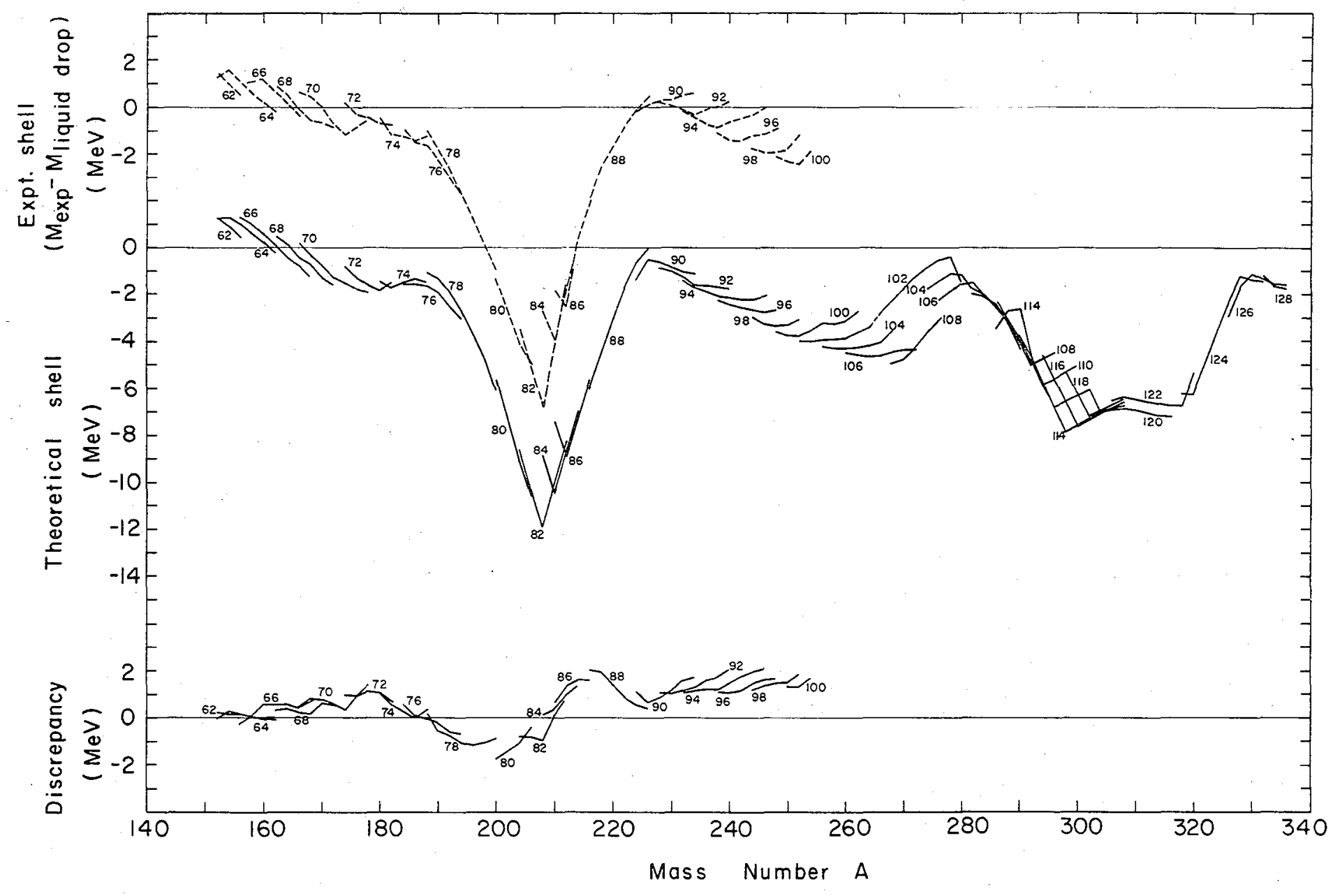




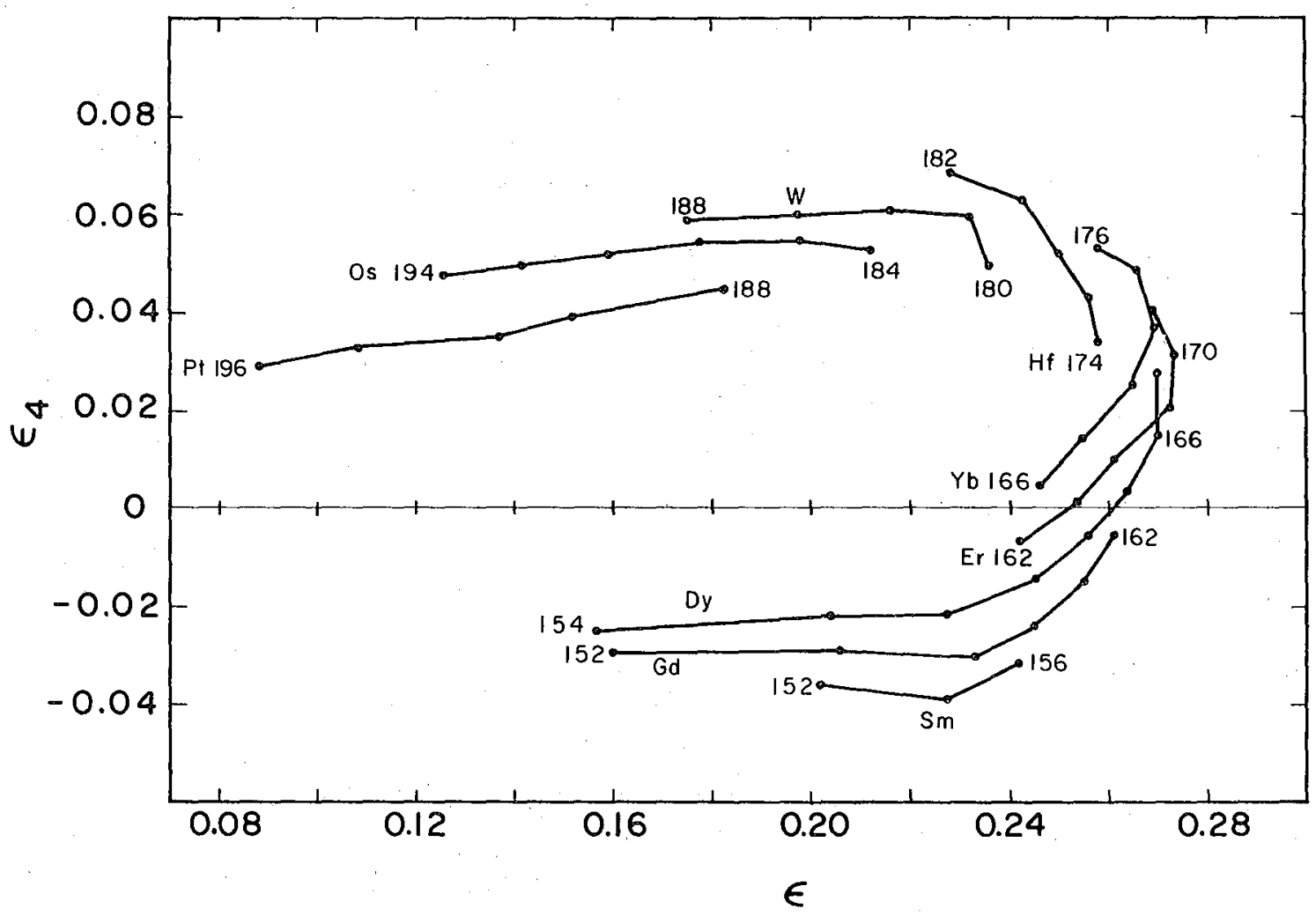

XBL687- 3439

Fig. 30 


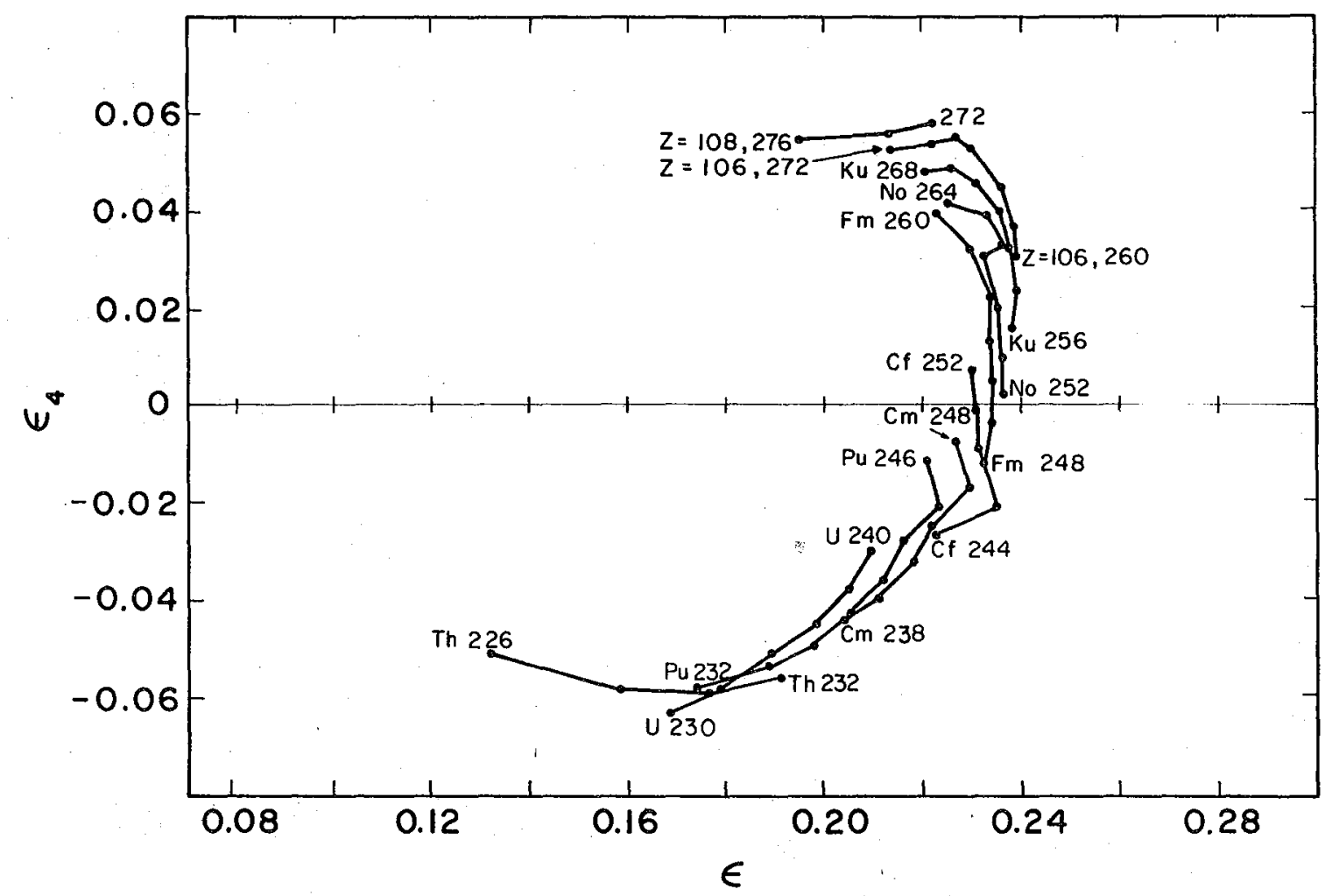

$\times 8 L 687-3438$

Fig. 31 


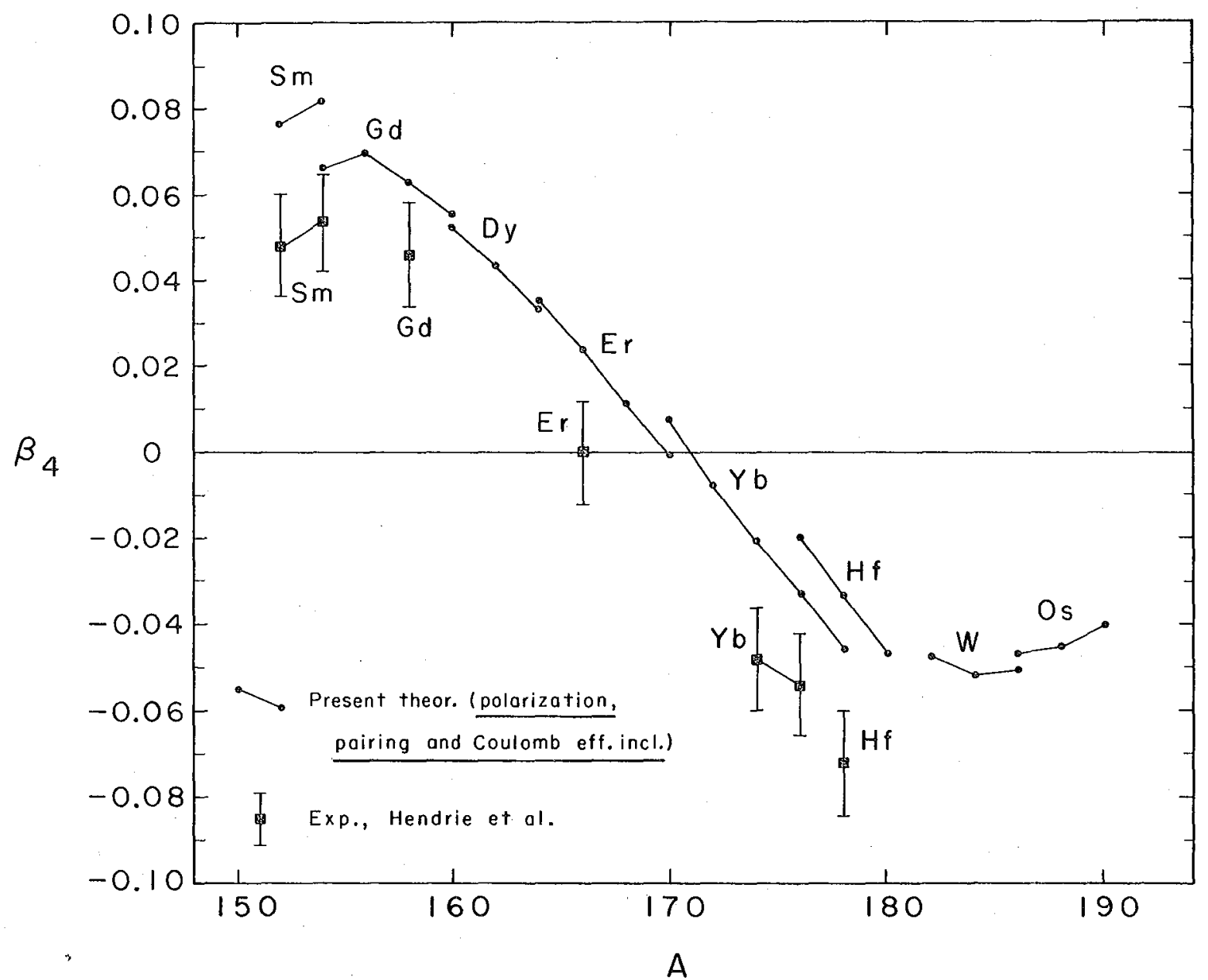

XBL688-3505

Fig. 32 


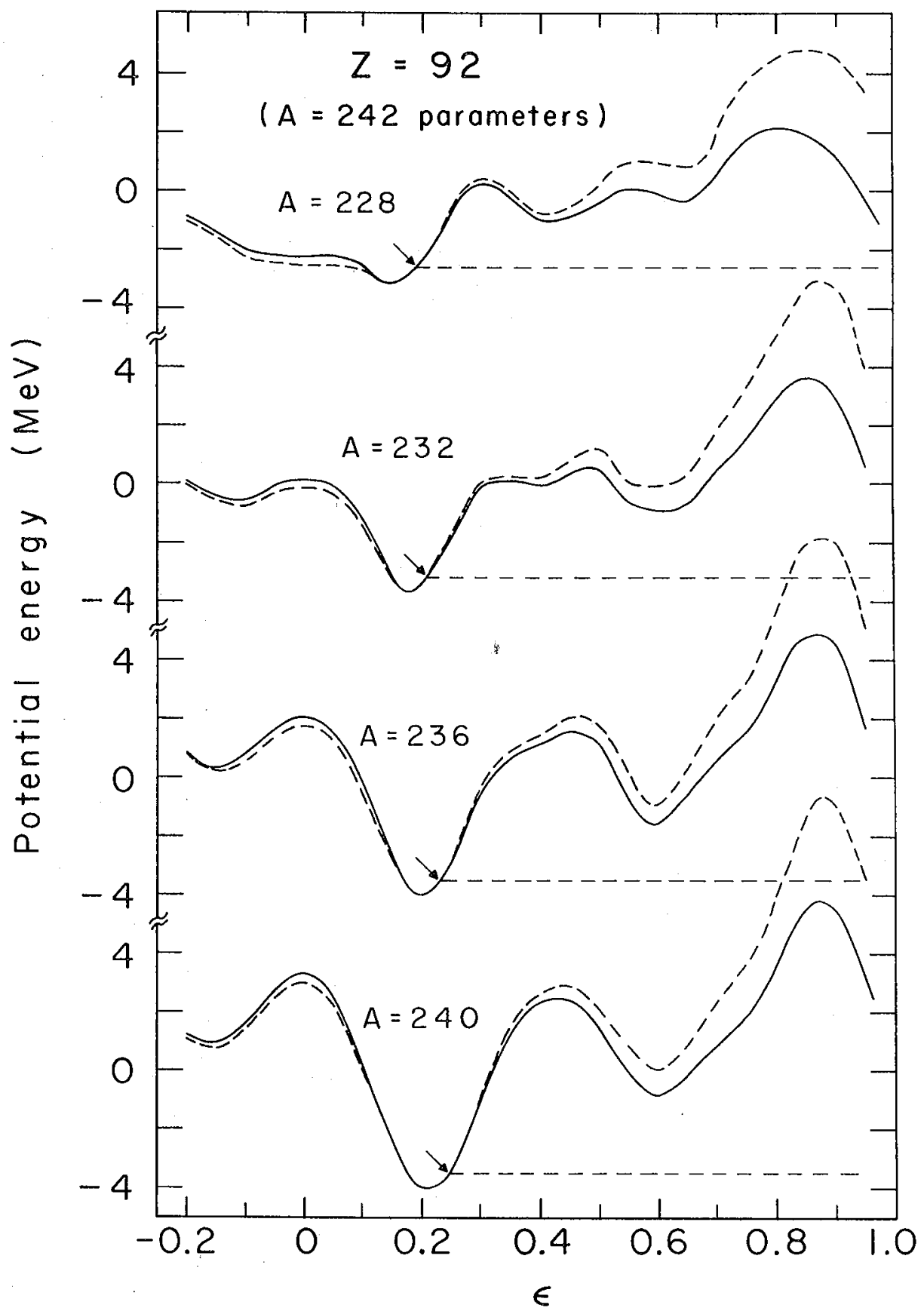

$X B L 688-3516$

Fig. 33a 


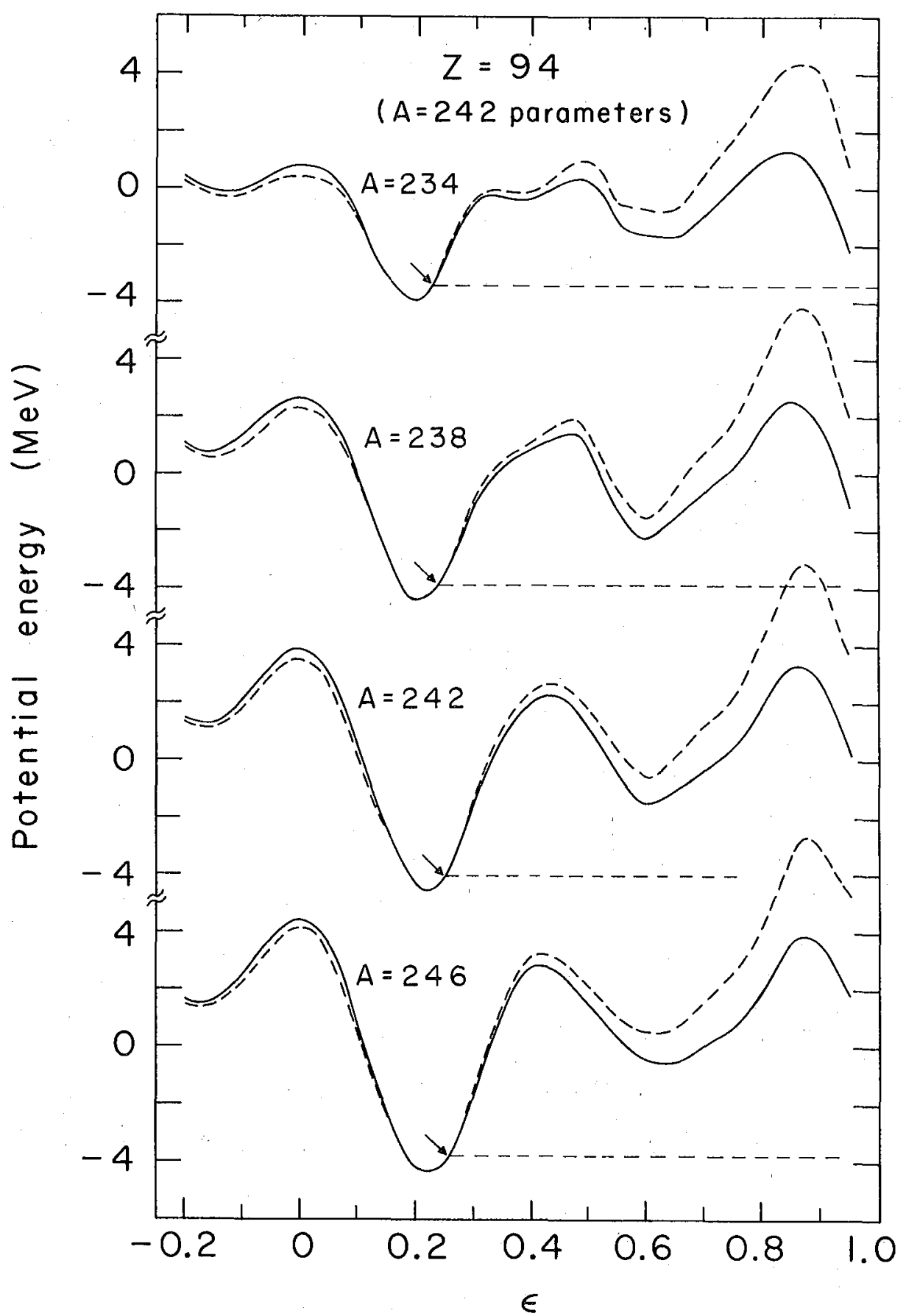

$X B L 688-3515$

Fig. 33b 
$-184-$

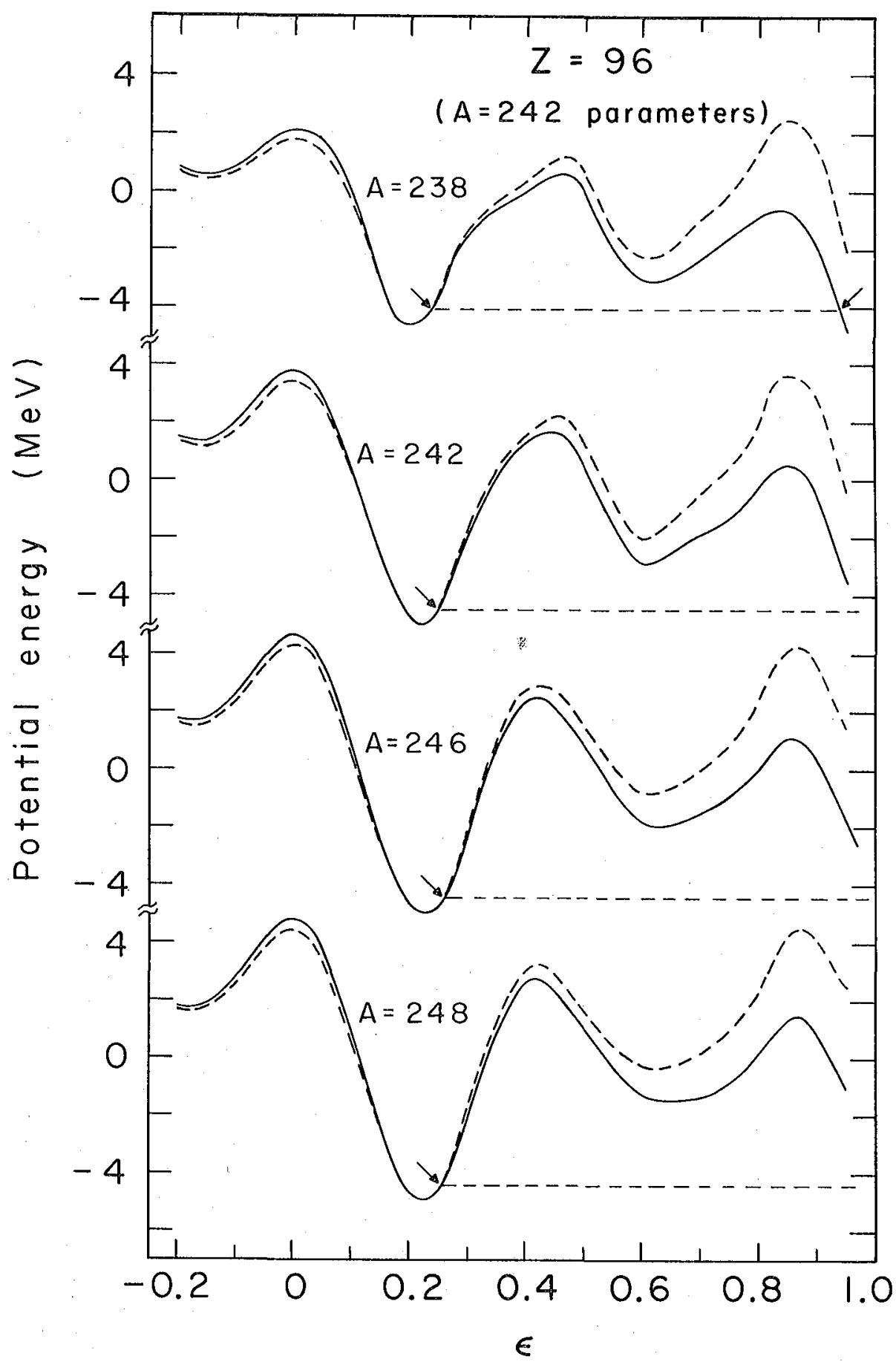

XBL688-3514

Fig. 33c 


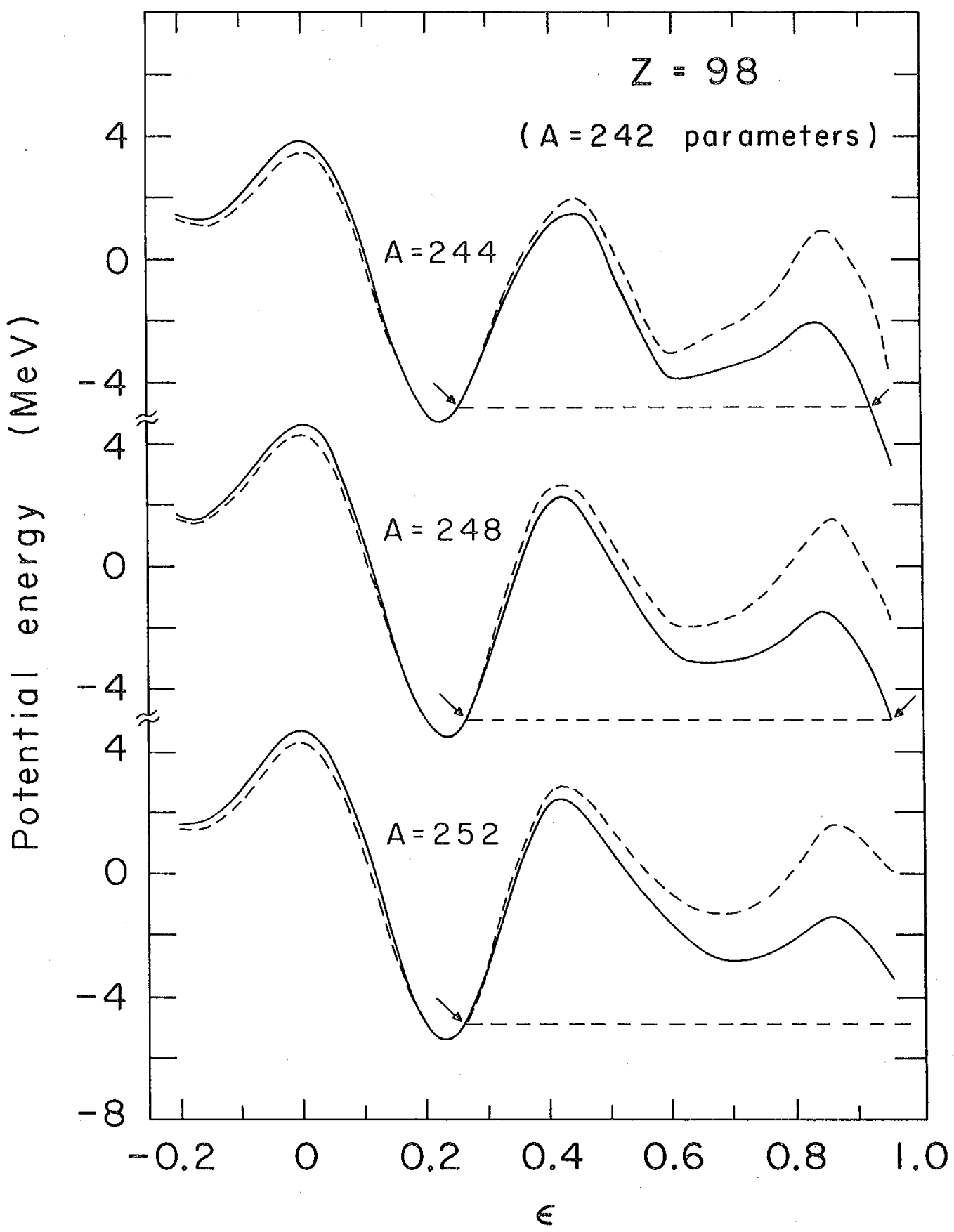

$X B L 688-3513$

Fig. $33 \mathrm{~d}$ 


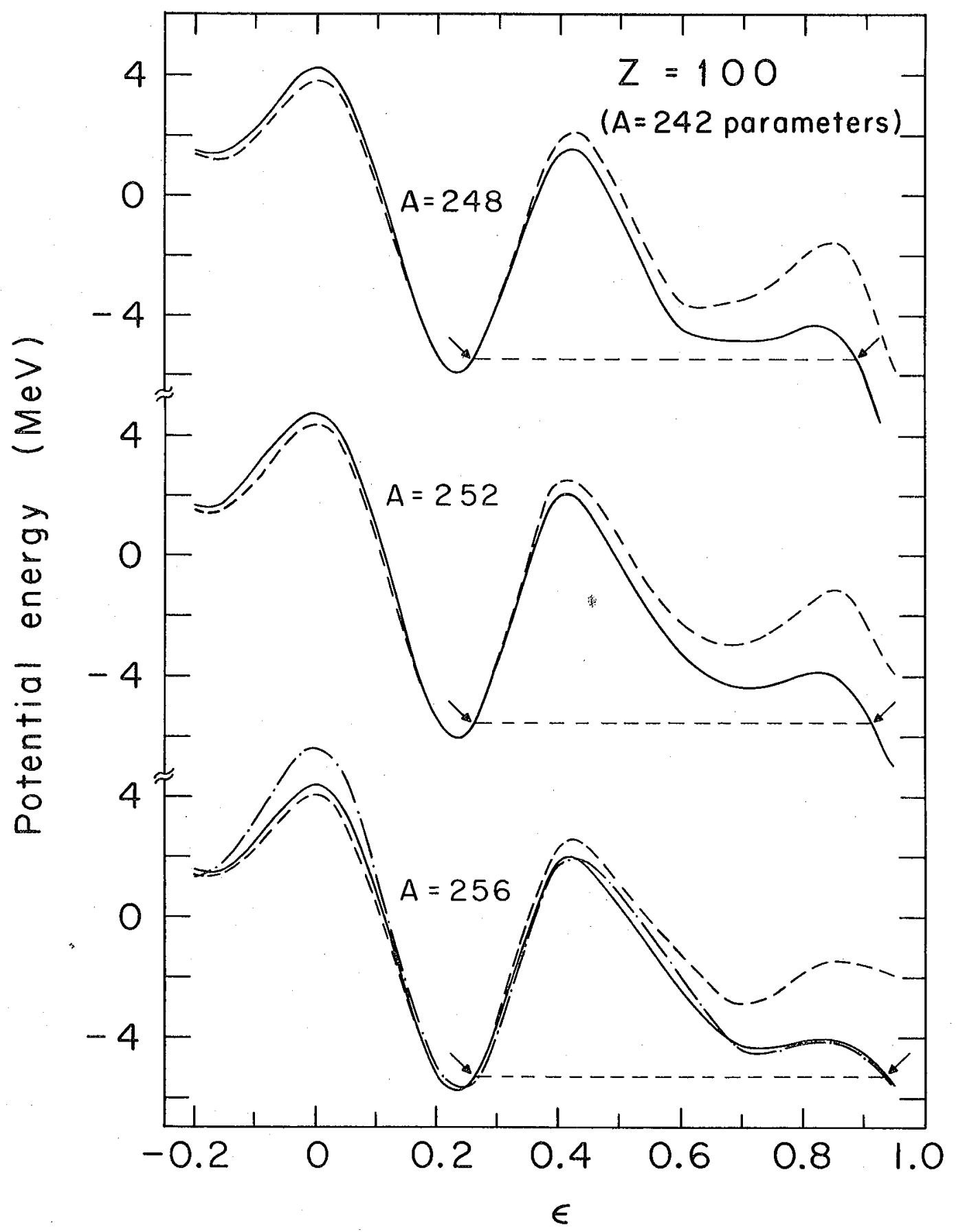

$X B L 688-3512$ 
$-187-$

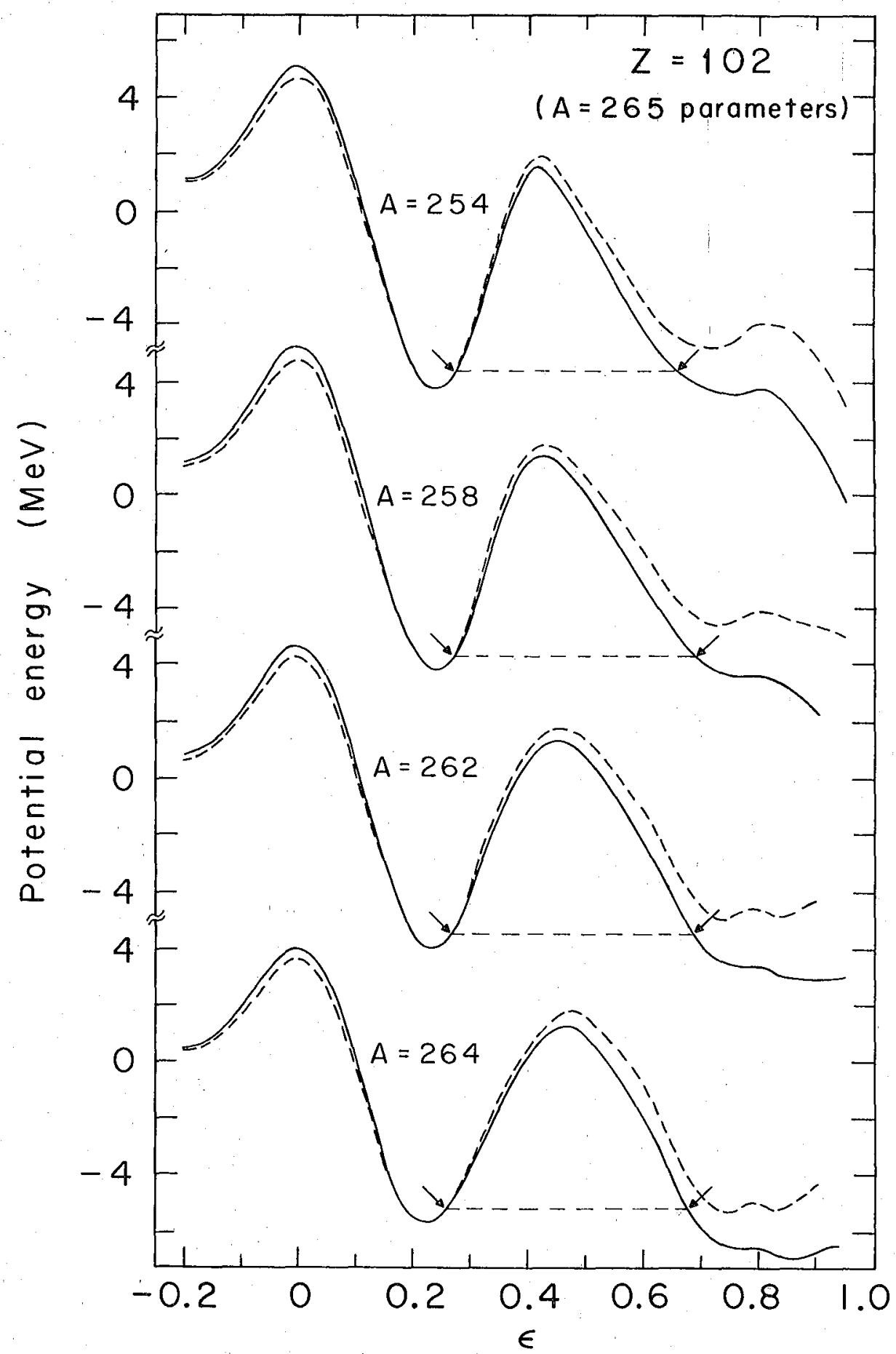

$X B L 688-3510$

Fig. $33 f$ 


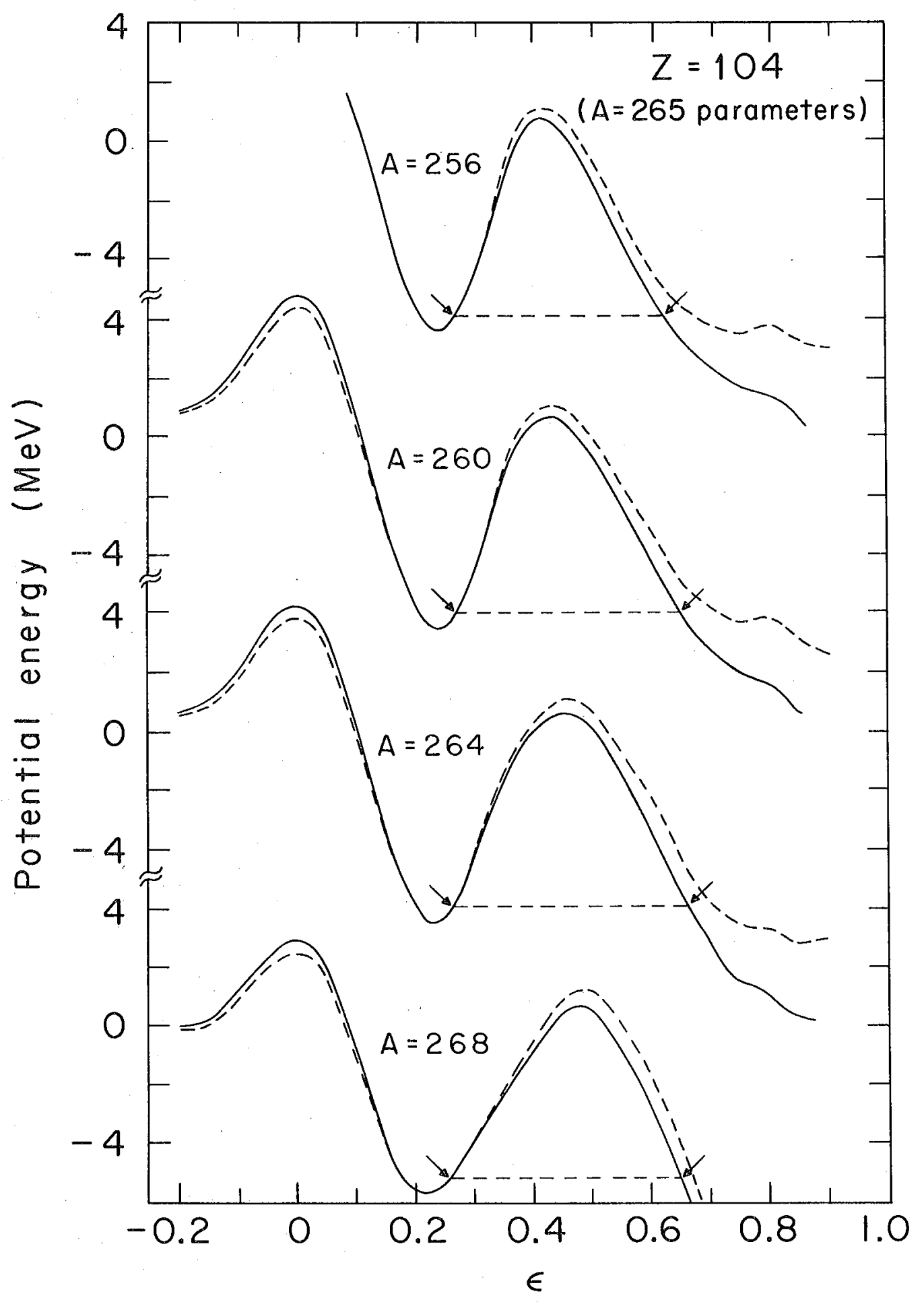

$X B L 688-3509$ 


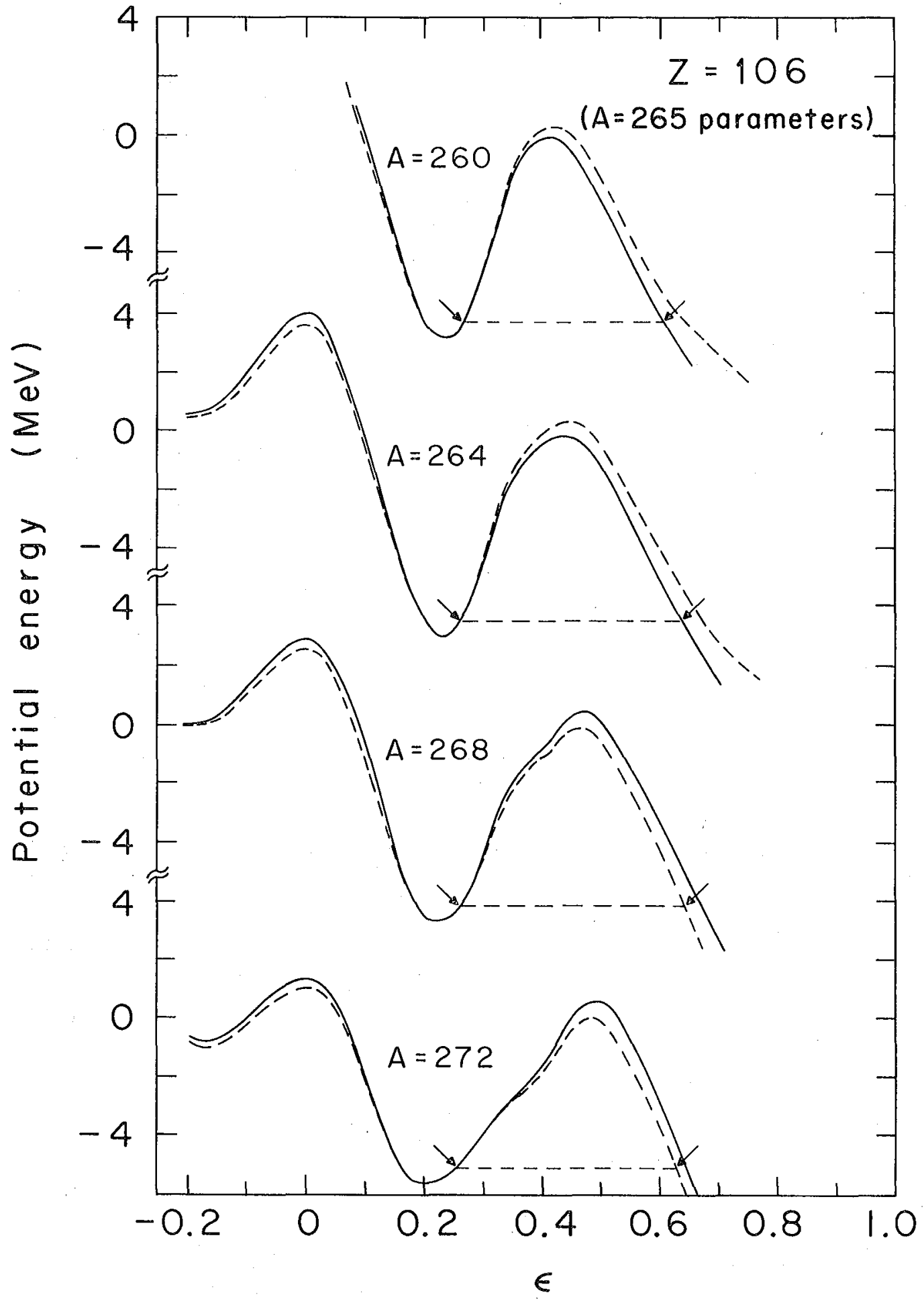

XBL688-3508

Fig. $33 h$ 
$-190-$

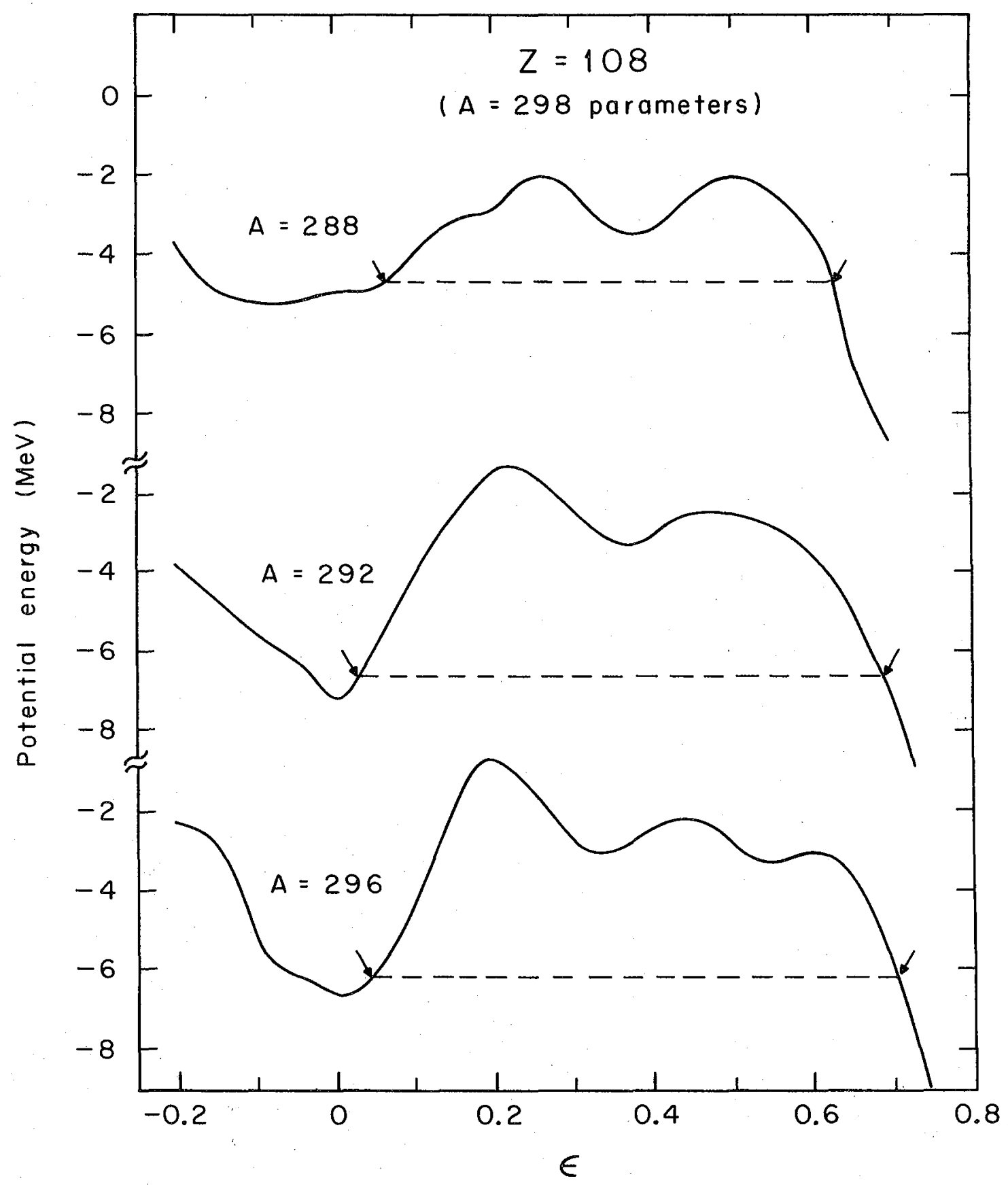

XBL688-3532

Fig. $33 i$ 


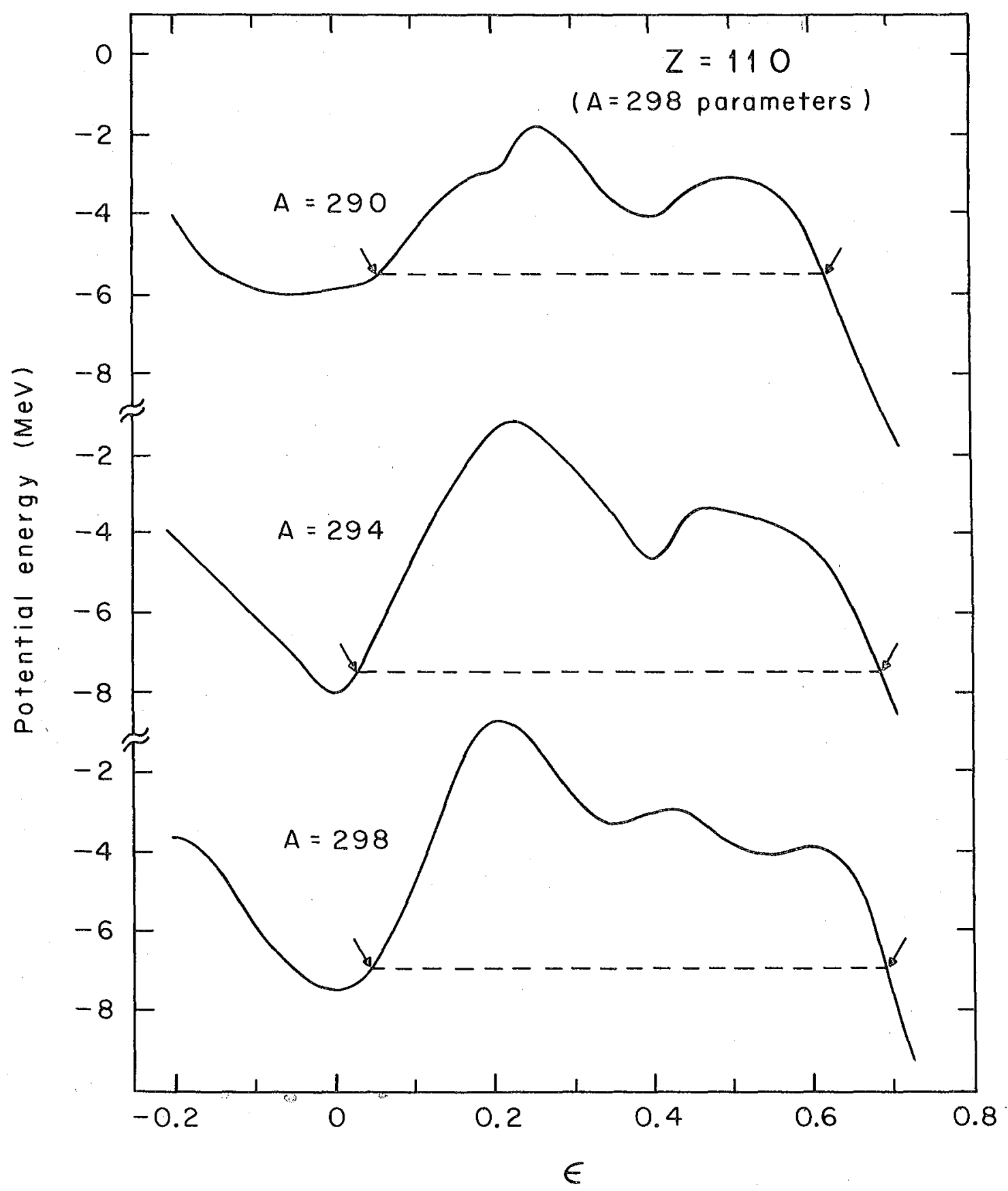

$X B L 688-3531$ 


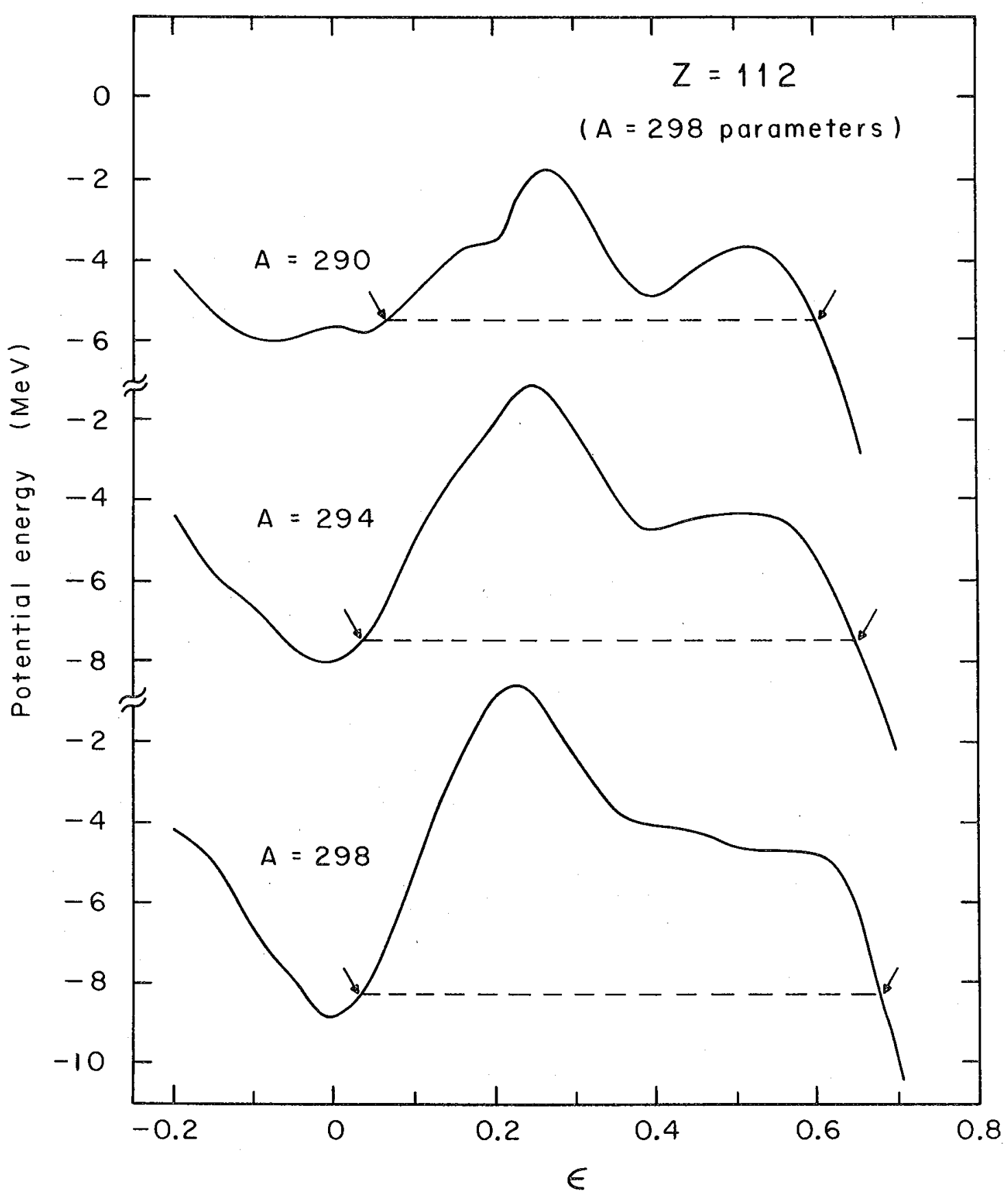

XBL688-3529

Fig. $33 \mathrm{k}$ 


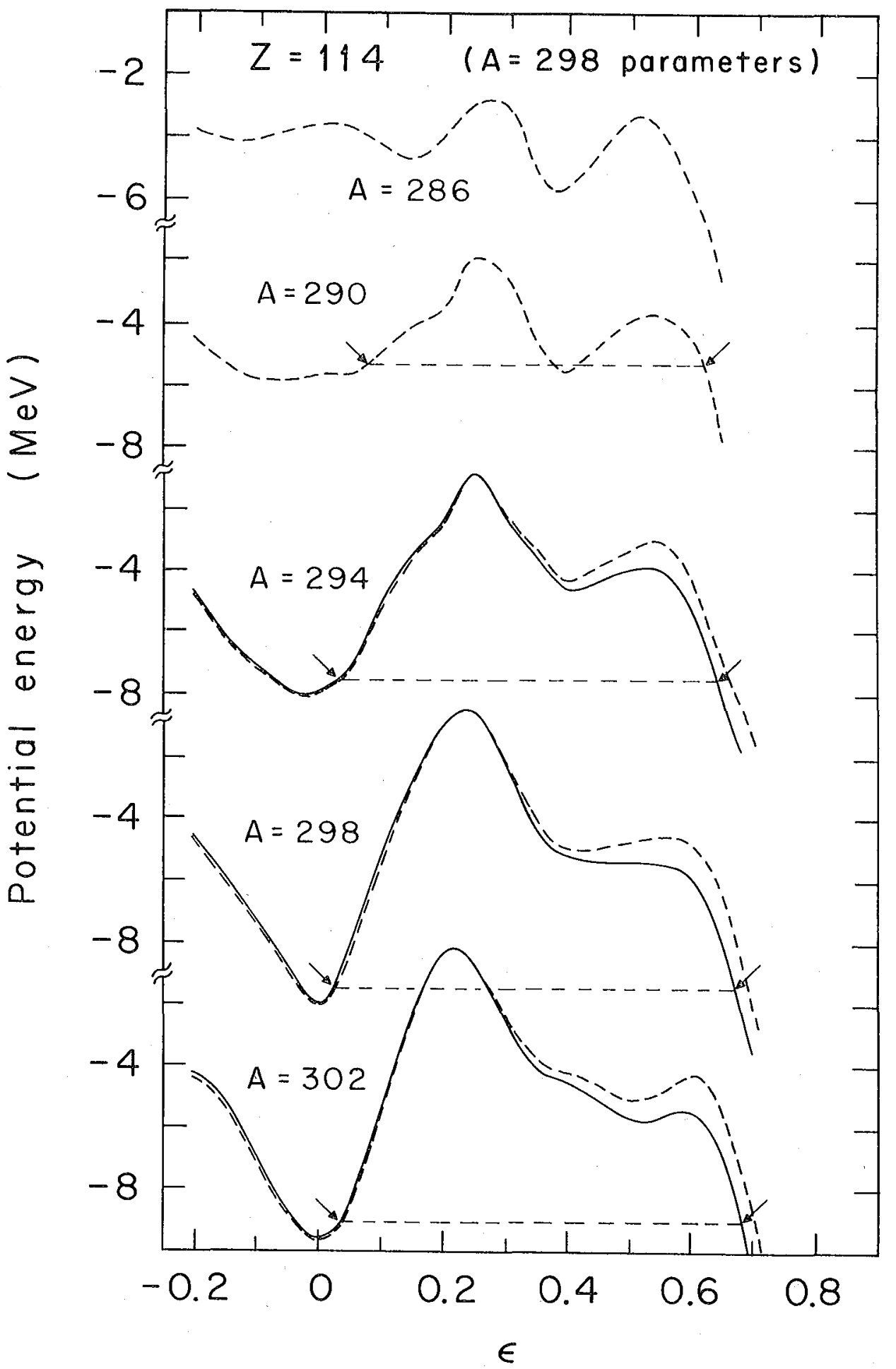

XBL688-3530

Fig. 33l 


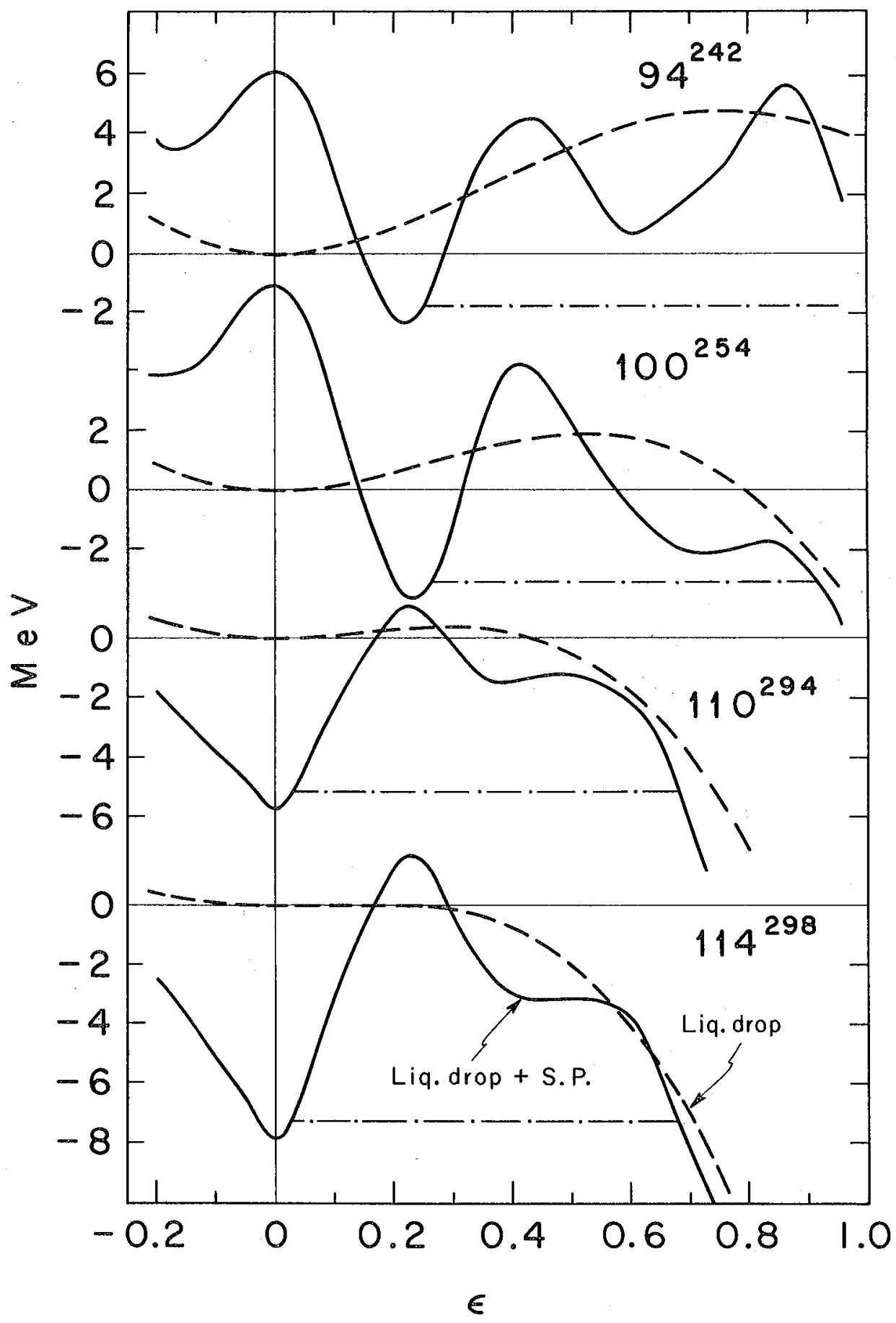

XBL688-3679

Fig. 34 


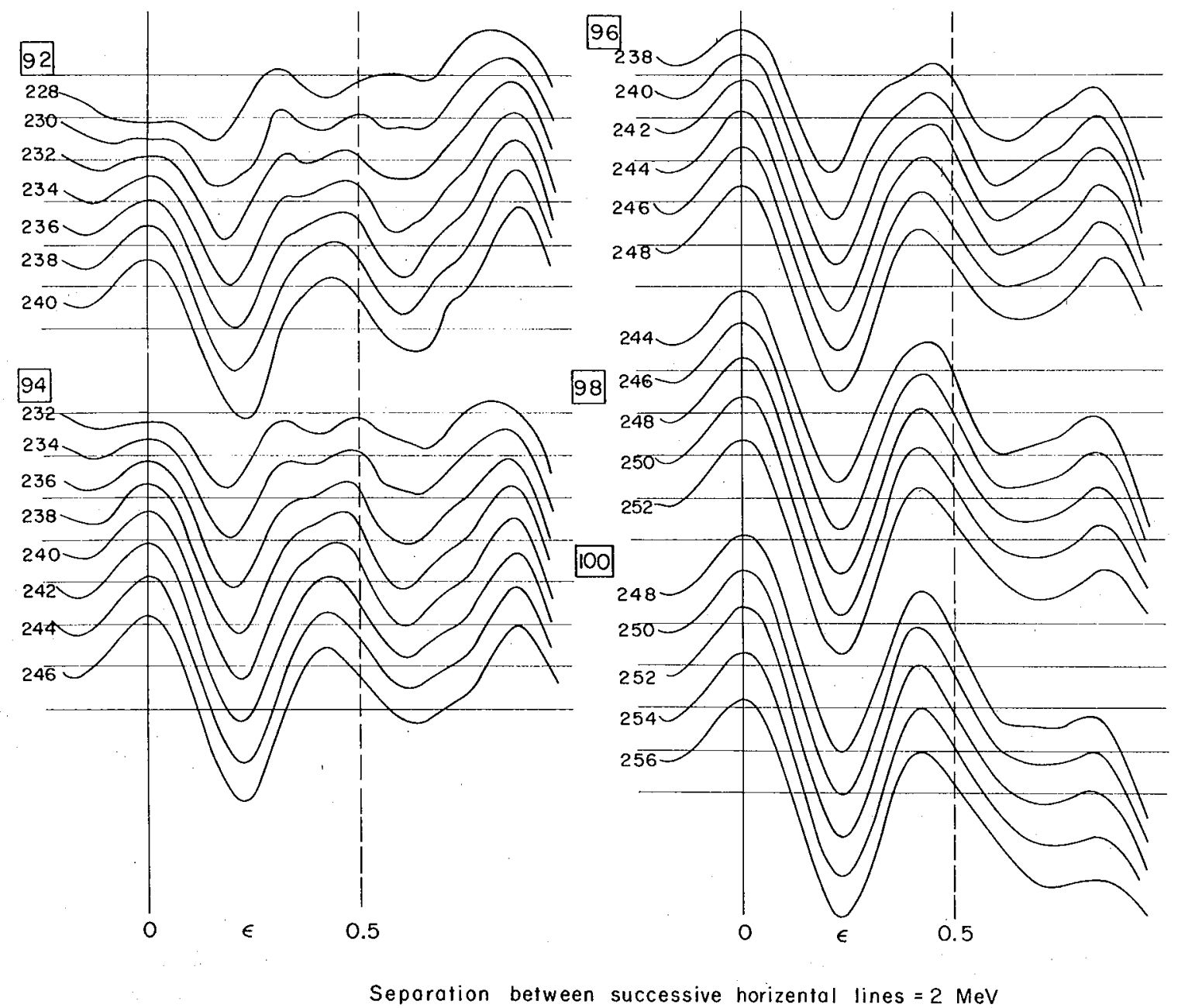

XBL69I - 1638

Fig. 35 


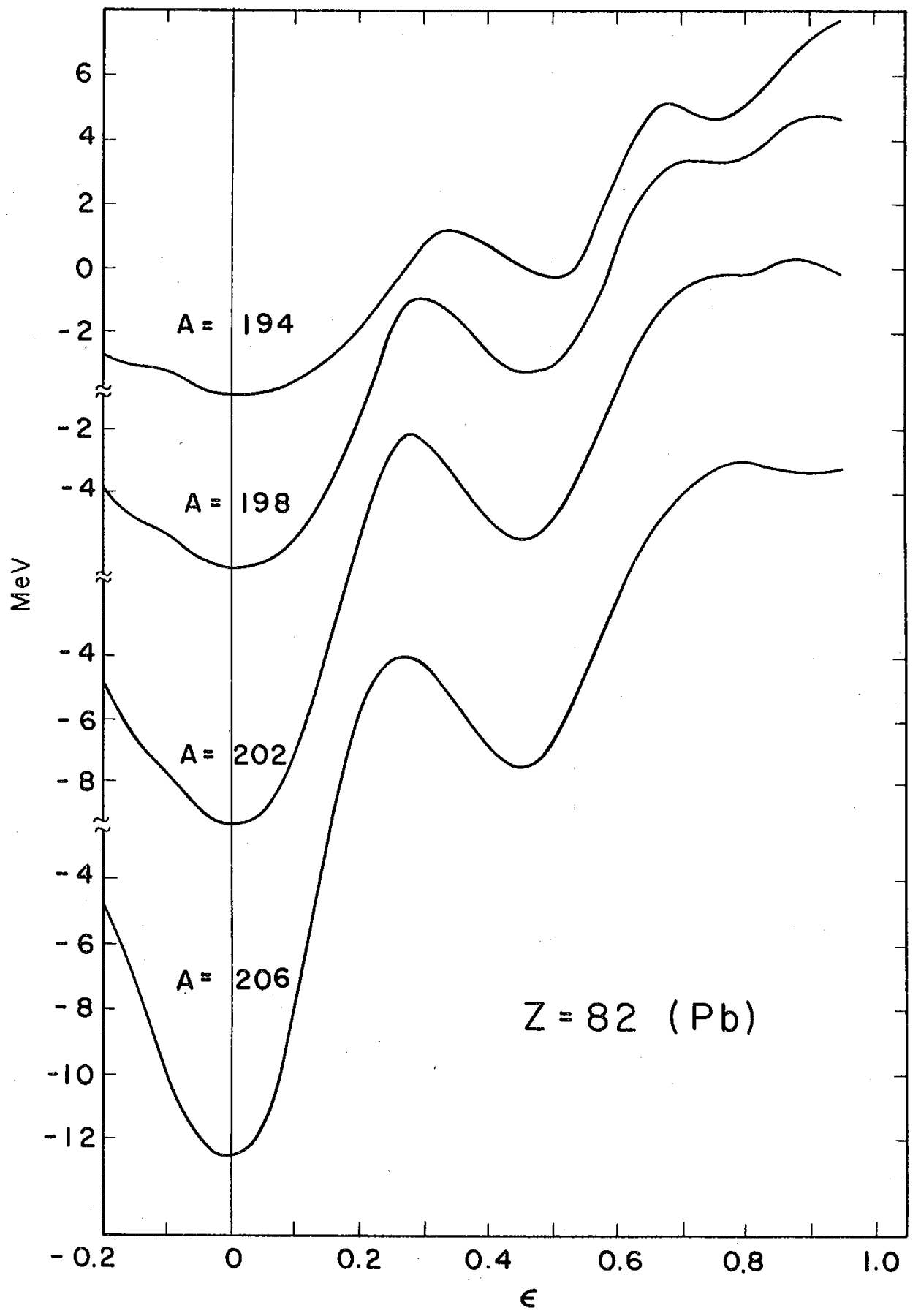

XHL $6.994-2+4.4$

Fig. 36a 


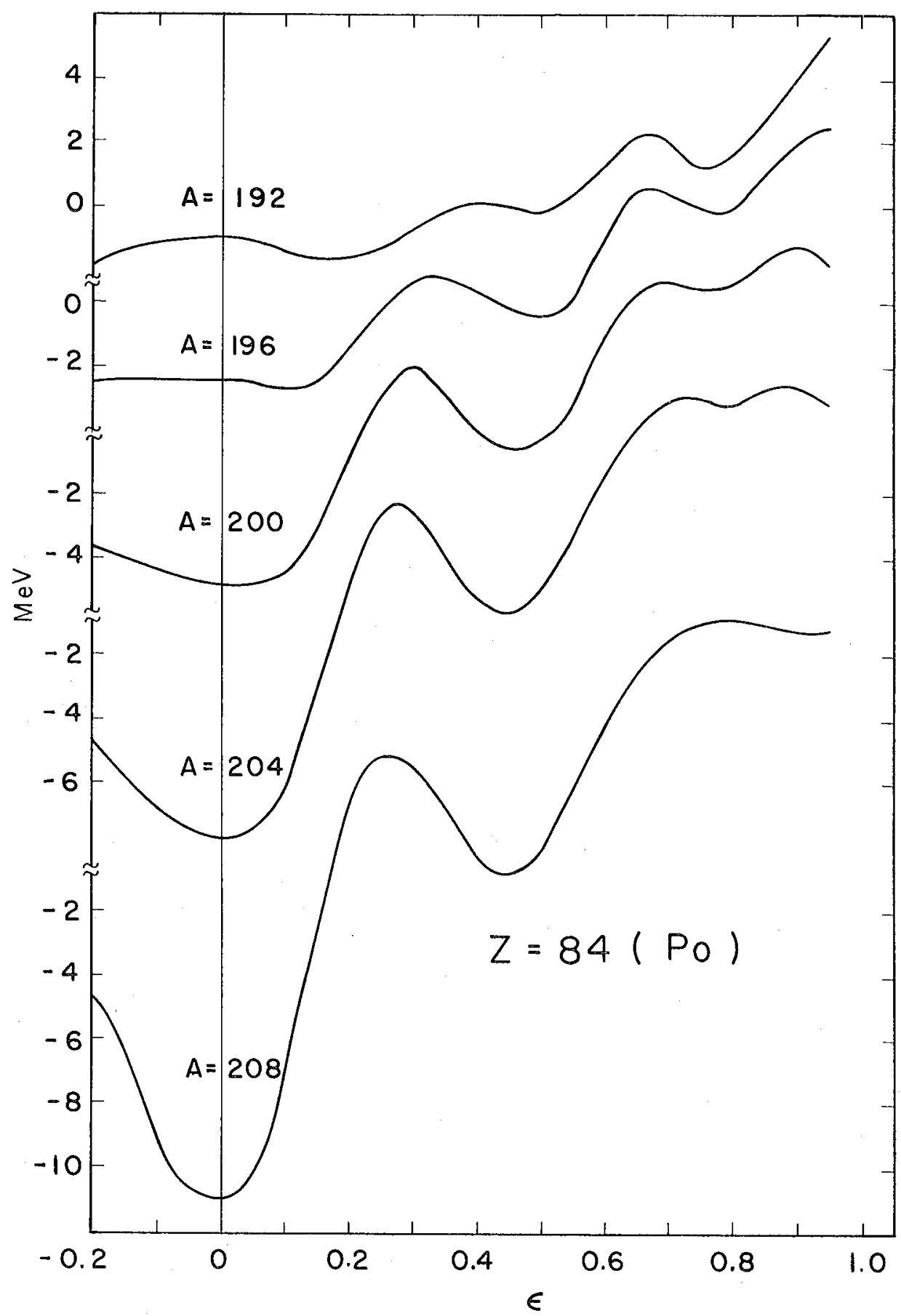

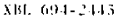

Fig. 36b 


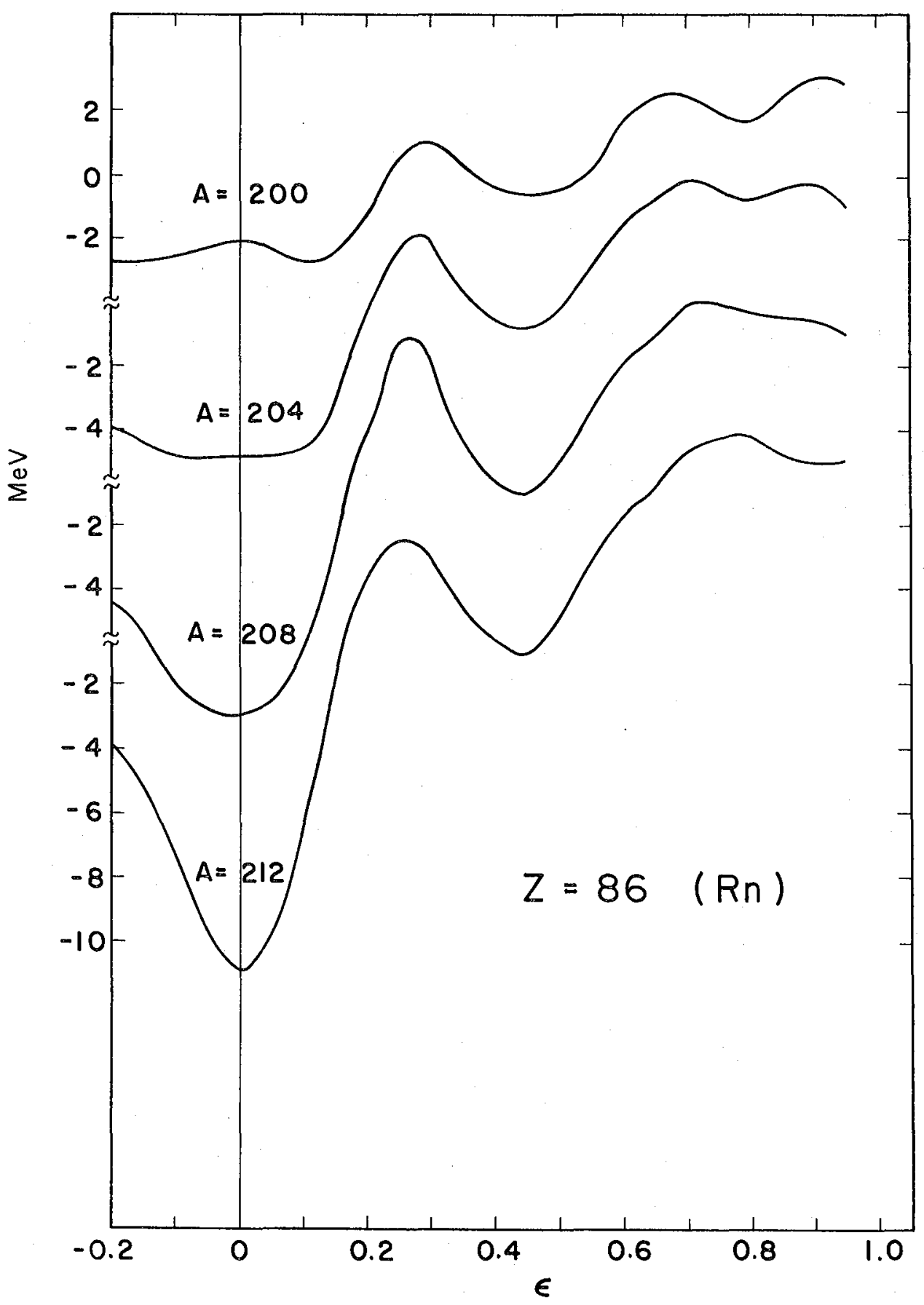

X31. (69) $4-24$

Fig. $36 \mathrm{c}$ 


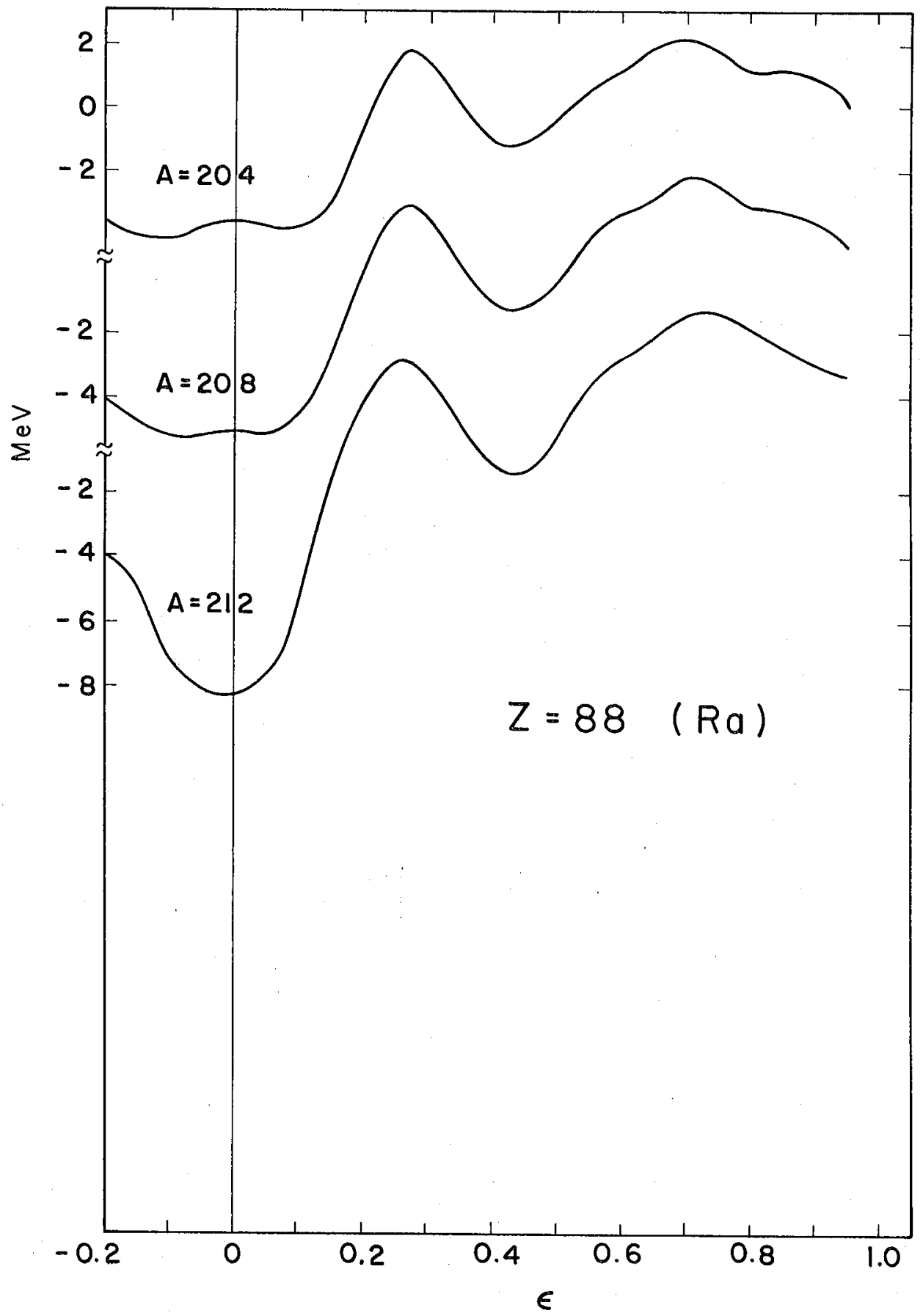

$x B L 693-2261$

Fig. 36d 


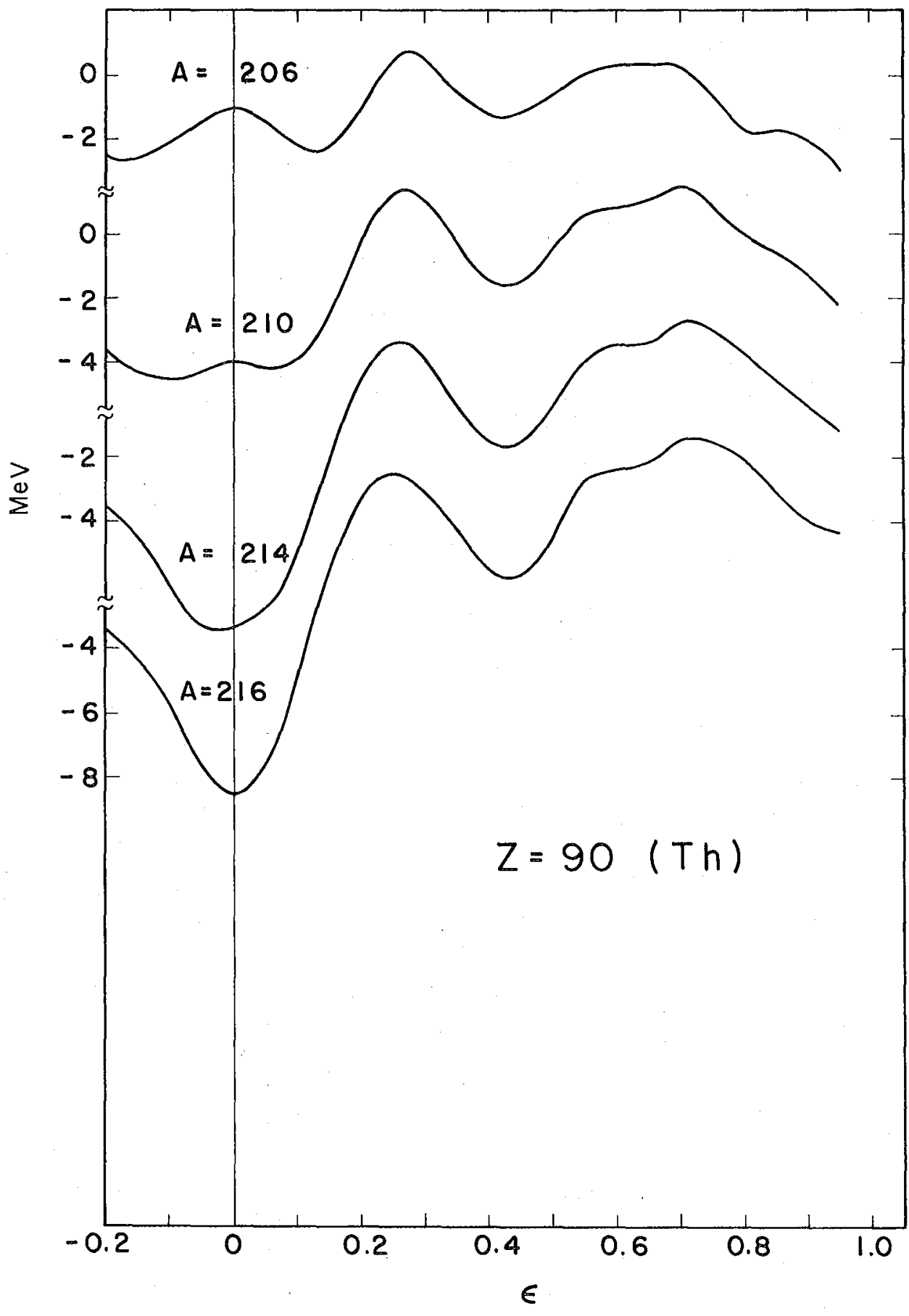

Fig. $36 \mathrm{e}$ 


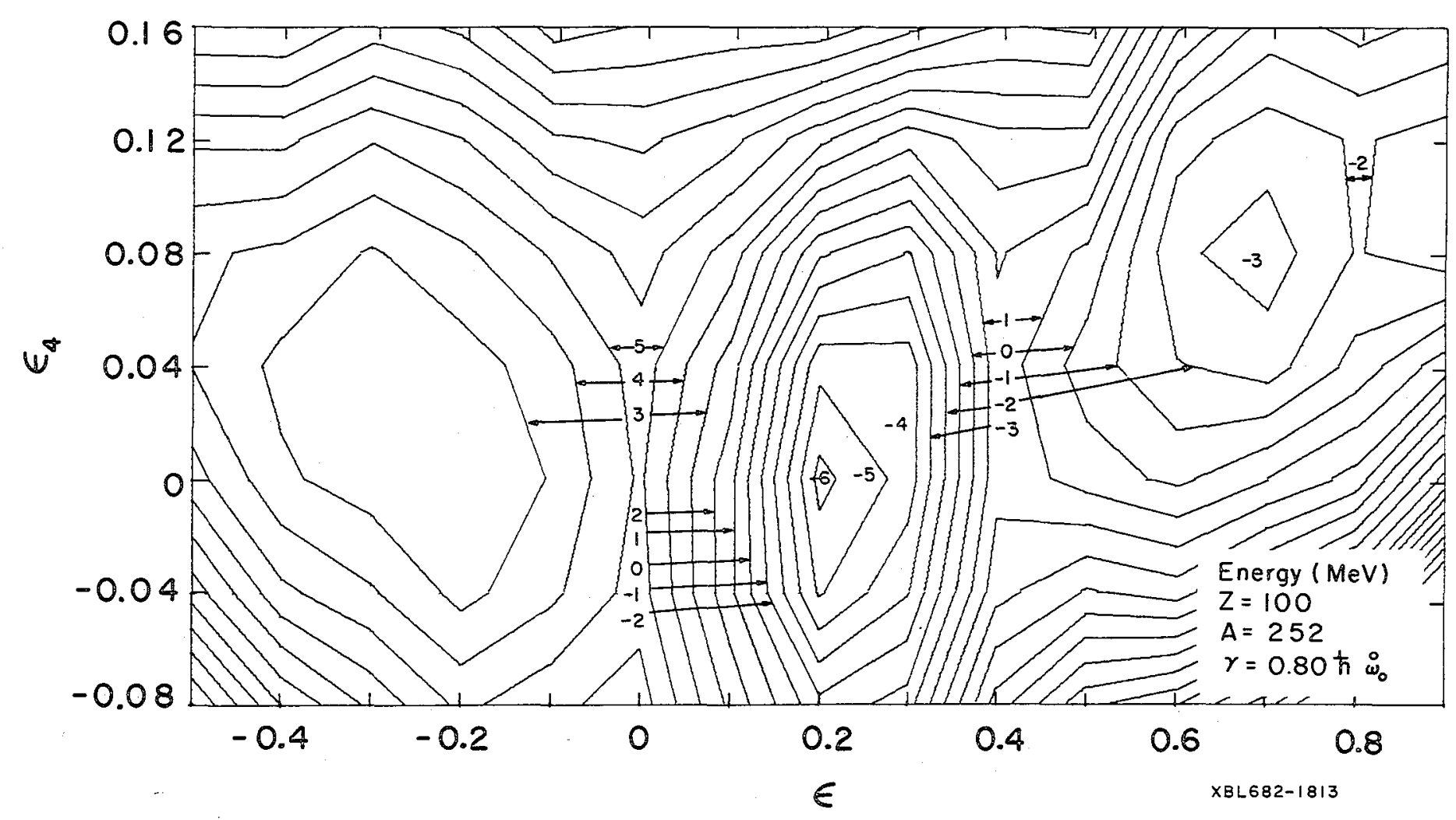




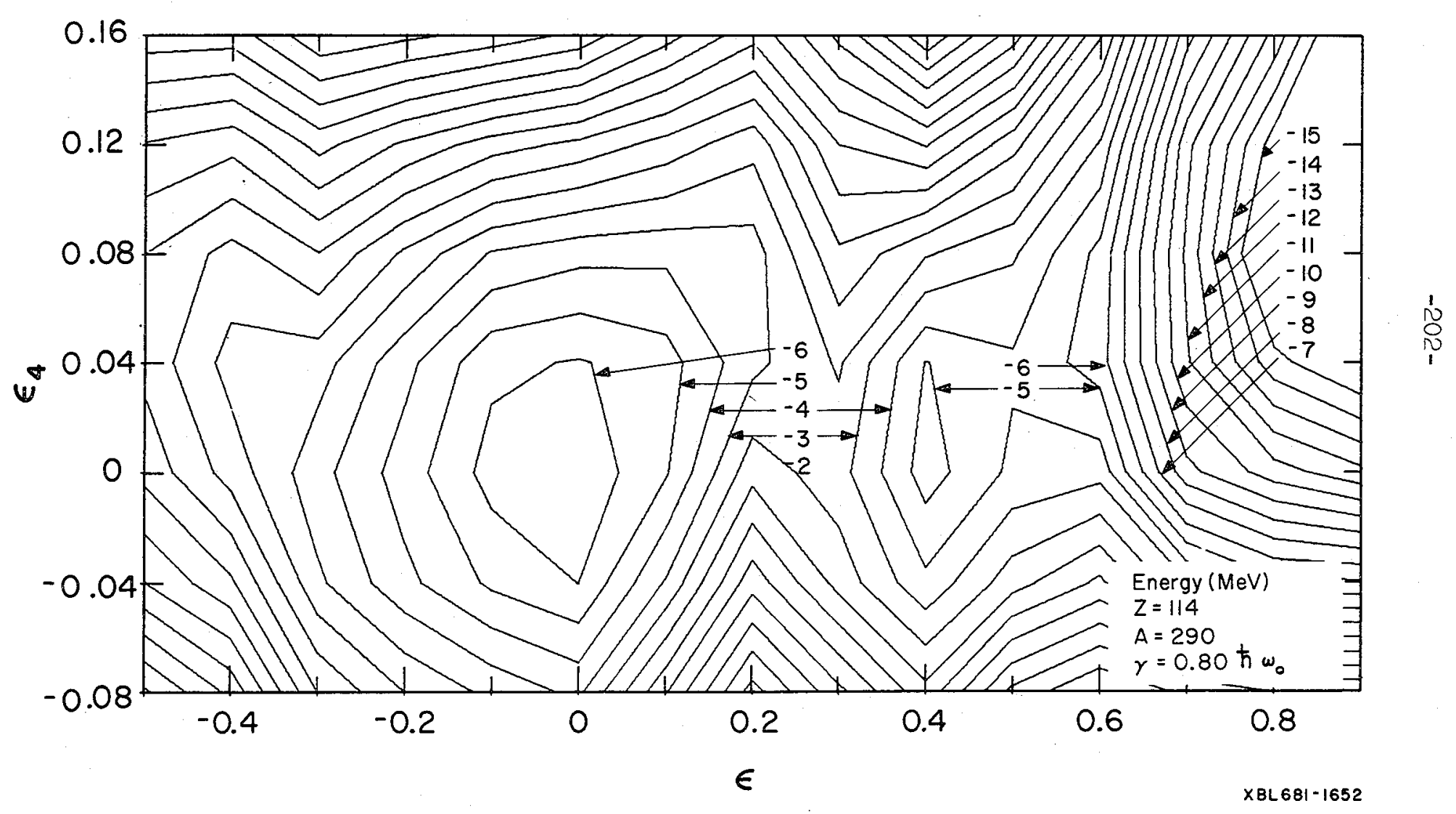

Fig. $37 \mathrm{~b}$ 


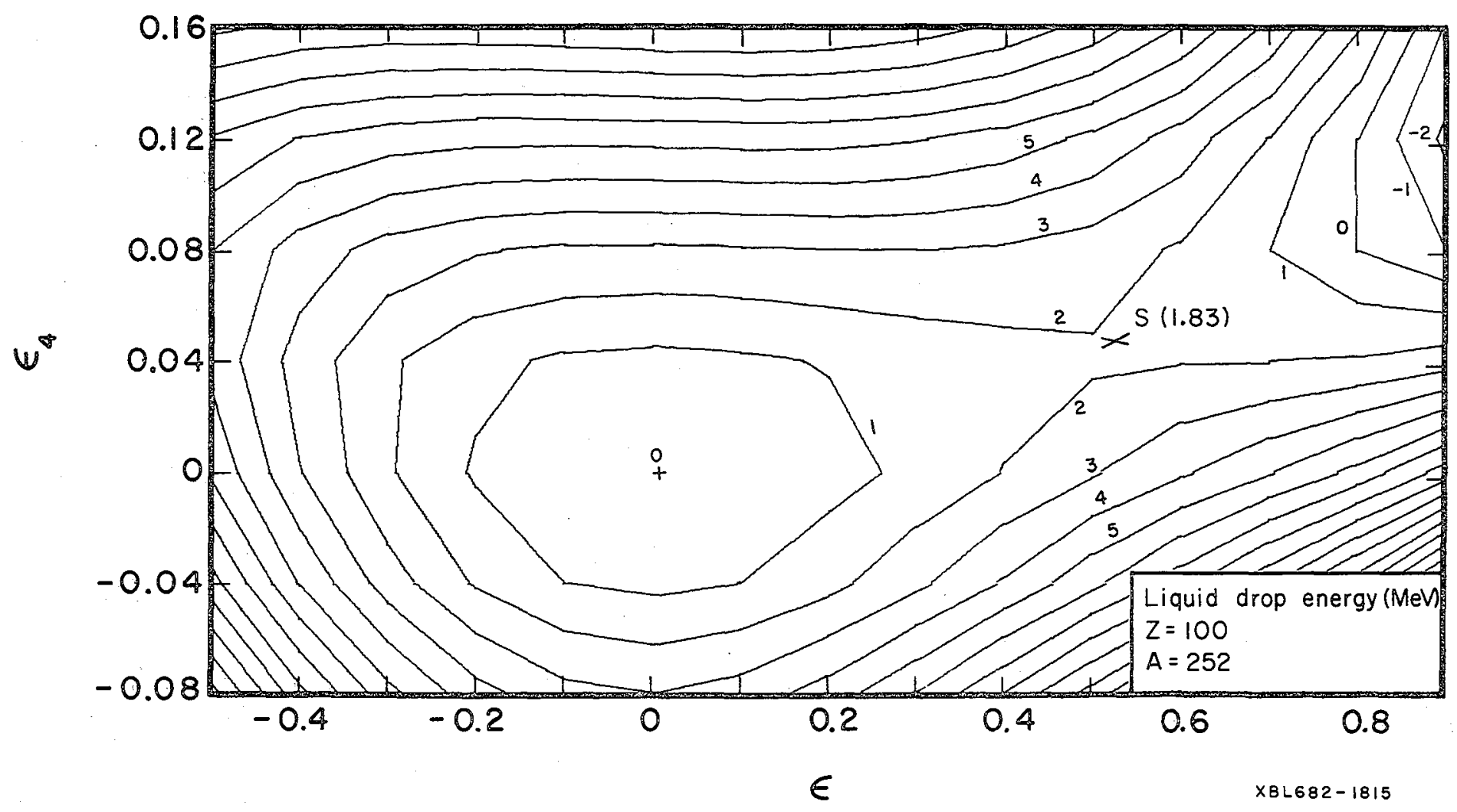

Fig. 38 a 


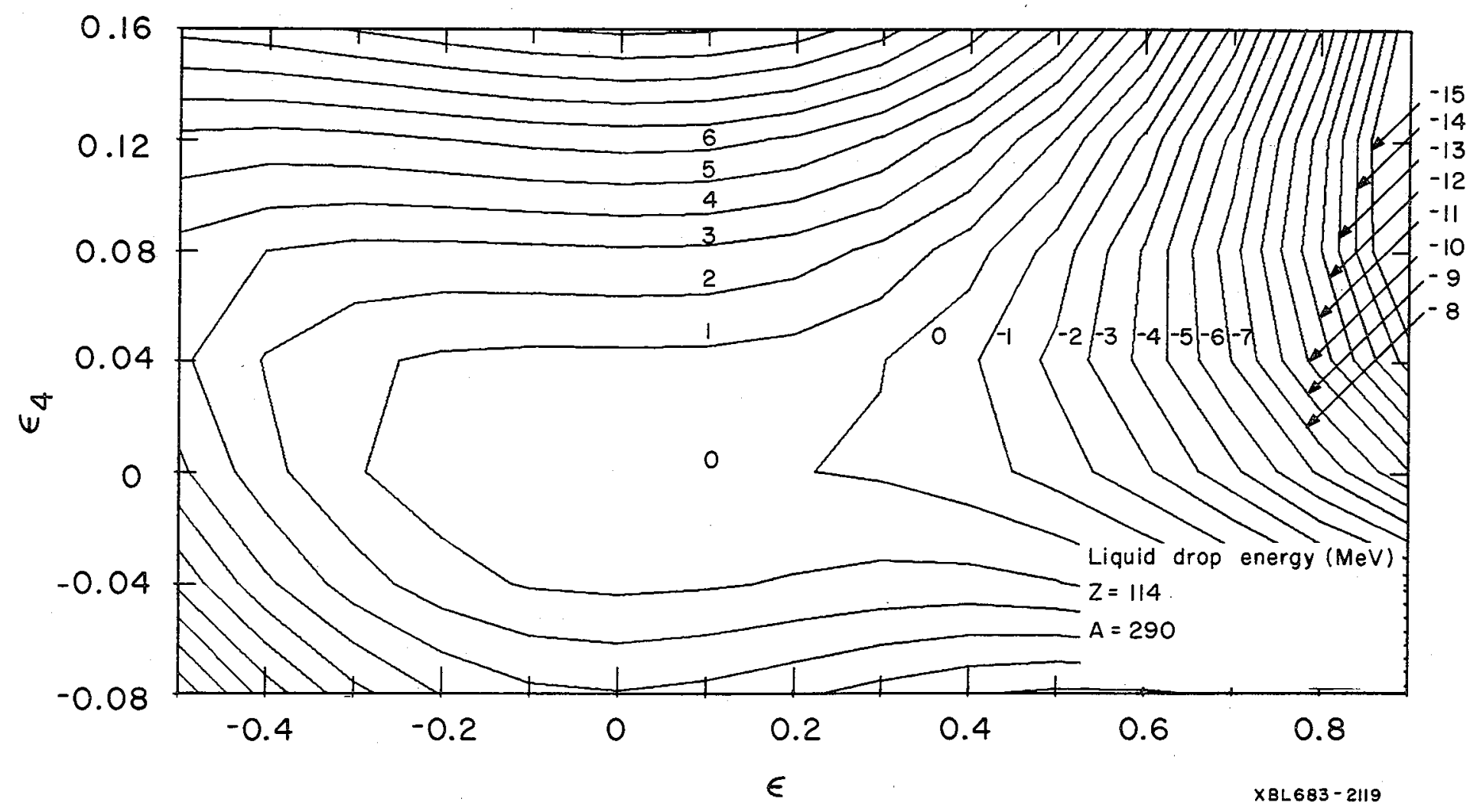

Fig. $38 b$ 


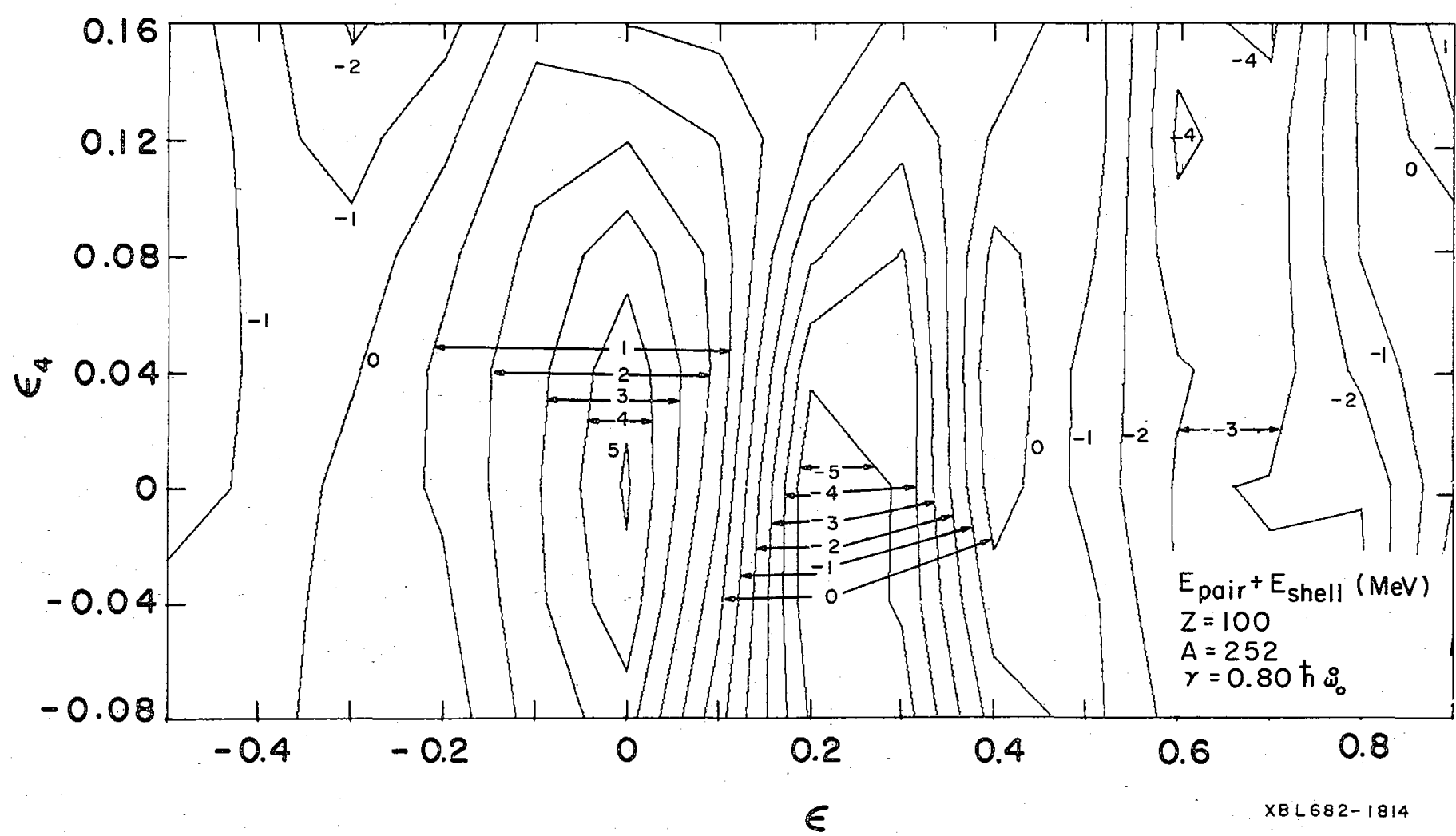

Fig. 39a 


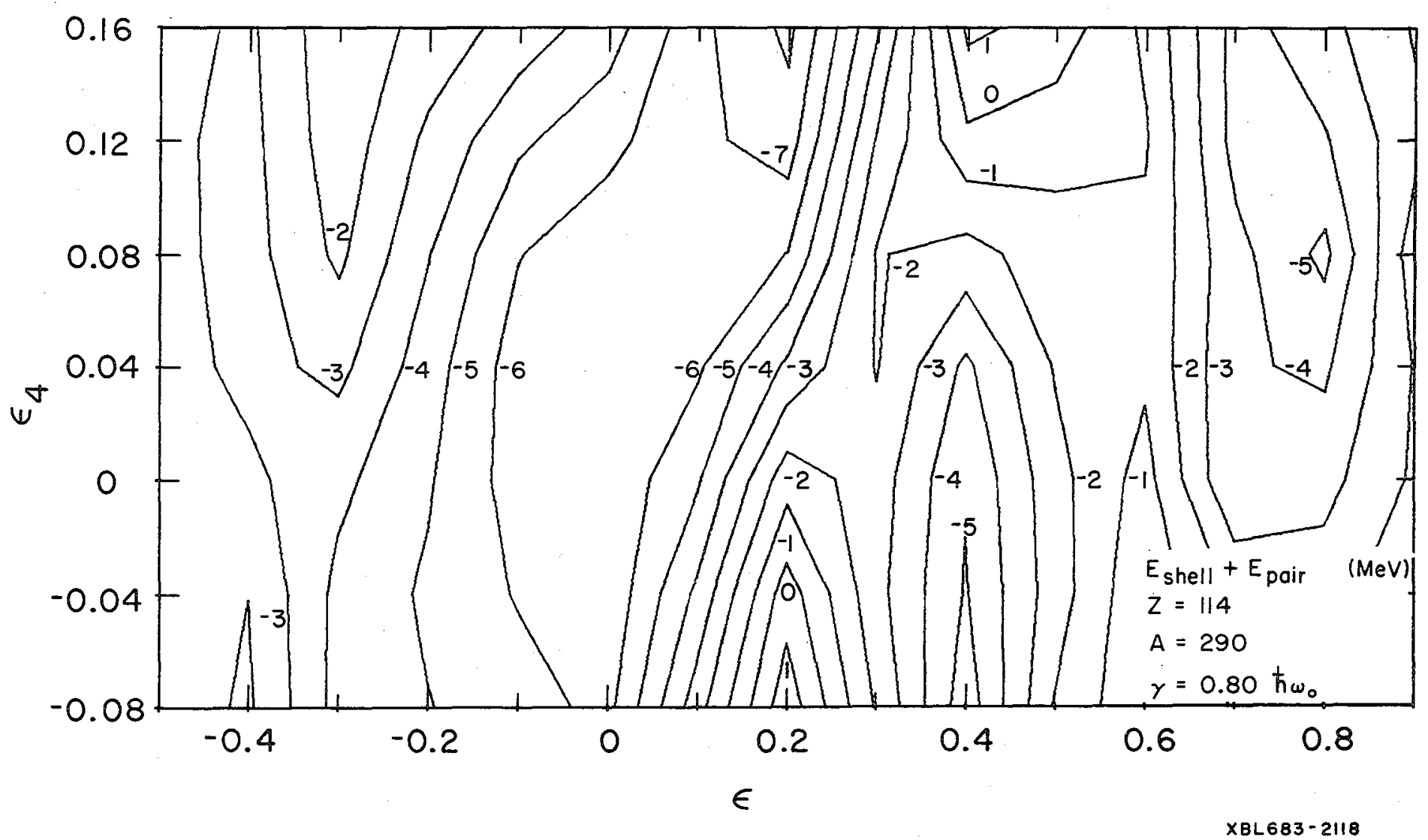

1
0
0
8

Fig. 390 


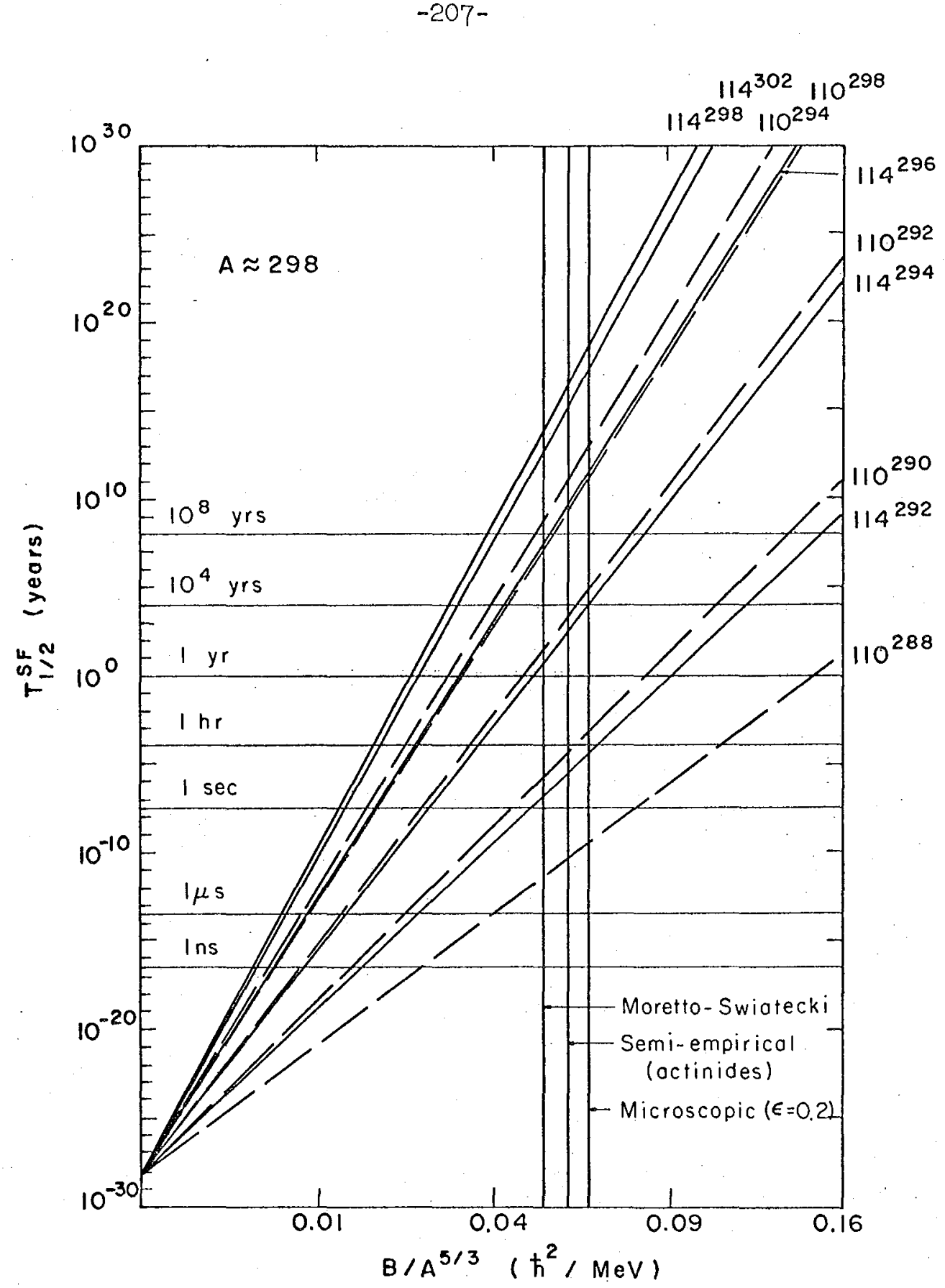

$X 8 L 691-1640$

Fig. 40 


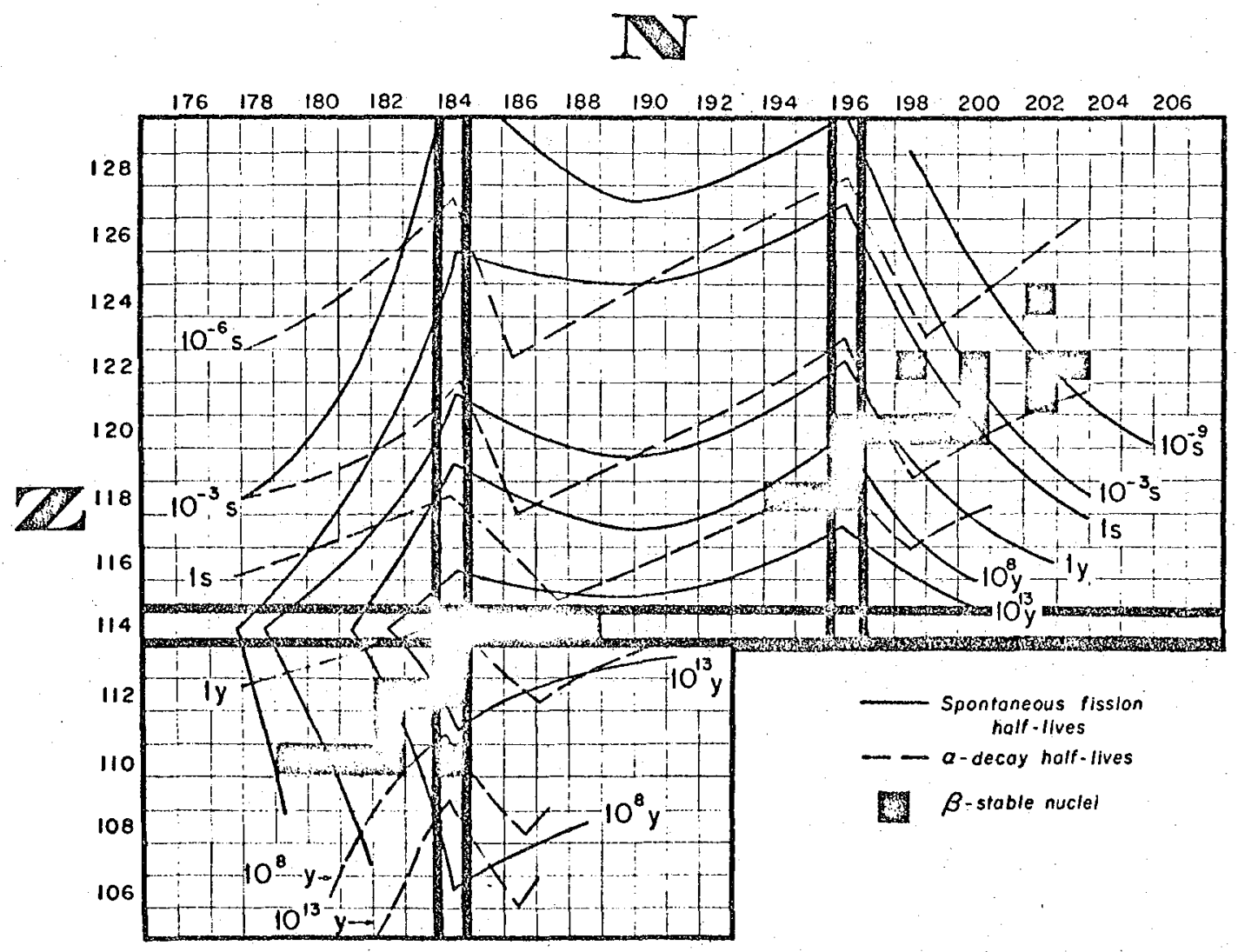

Fig. 4 I 

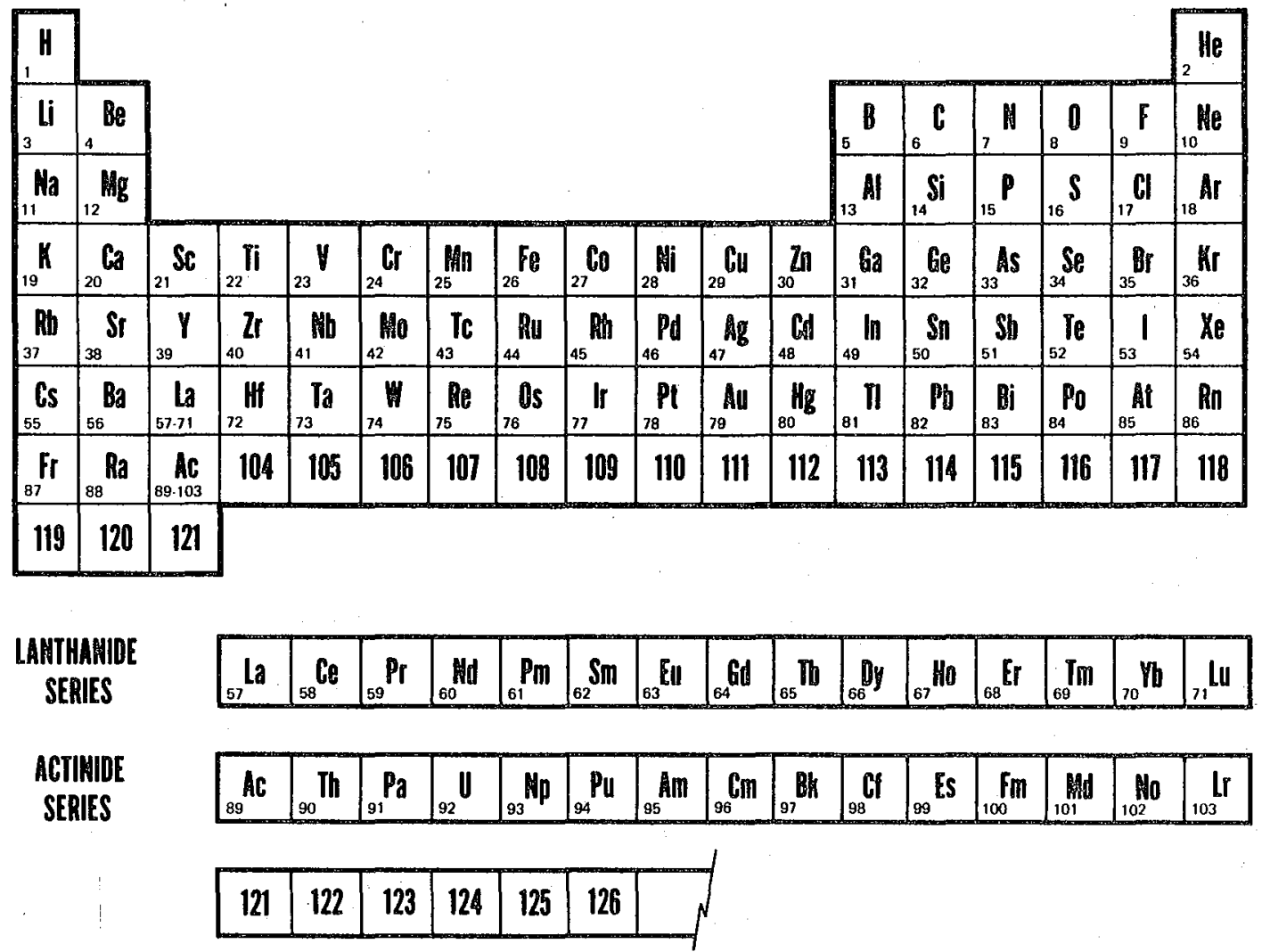
, 
This report was prepared as an account of Government sponsored work. Neither the United States, nor the Commission, nor any person acting on behalf of the Commission:

A. Makes any warranty or representation, expressed or implied, with respect to the accuracy, completeness, or usefulness of the information contained in this report, or that the use of any information, apparatus, method, or process disclosed in this report may not infringe privately owned rights; or

B. Assumes any liabilities with respect to the use of, or for damages resulting from the use of any information, apparatus, method, or process disclosed in this report.

As used in the above, "person acting on behalf of the Commission" includes any employee or contractor of the Commission, or employee of such contractor, to the extent that such employee or contractor of the Commission, or employee of such contractor prepares, disseminates, or provides access to, any information pursuant to his employment or contract with the Commission, or his employment with such contractor. 